\title{
Cannabis: A Treasure Trove or Pandora's Box? ${ }^{\#}$
}

\author{
Katalin Solymosi ${ }^{1}$ and Attila Köfalvi ${ }^{2,3 *}$ \\ ${ }^{1}$ Department of Plant Anatomy, Institute of Biology, Eötvös Loránd University, Budapest, Hungary; \\ ${ }^{2}$ Laboratory of Neuromodulation and Metabolism, CNC - Center for Neuroscience and Cell Biology of Coimbra, University of \\ Coimbra, Coimbra, Portugal \\ ${ }^{3}$ Institute for Interdisciplinary Research, University of Coimbra, Coimbra, Portugal
}

\begin{abstract}
Cannabis is one of the earliest cultivated plants. Cannabis of industrial utility and culinary value is generally termed as hemp. Conversely, cannabis that is bred for medical, spiritual and recreational purposes is called marijuana. The female marijuana plant produces a significant quantity of bio- and psychoactive phytocannabinoids, which regained the spotlight with the discovery of the endocannabinoid system of the animals in the early 90 's. Nevertheless, marijuana is surrounded by controversies, debates and misconceptions related to its taxonomic classification, forensic identification, medical potential, legalization and its long-term health consequences. In the first part, we provide an in-depth review of the botany and taxonomy of Cannabis. We then overview the biosynthesis of phytocannabinoids within the glandular trichomes with emphasis on the role of peculiar plastids in the production of the secreted material. We also compile the analytical methods used to determine the phytocannabinoid composition of glandular trichomes. In the second part, we revisit the psychobiology and molecular medicine of marijuana. We summarize our current knowledge on the recreational use of cannabis with respect to the modes of consumption, short-term effects, chronic health consequences and cannabis use disorder. Next, we overview the molecular targets of a dozen major and minor bioactive cannabinoids in the body. This helps us introduce the endocannabinoid system in an unprecedented detail: its up-to-date molecular biology, pharmacology, physiology and medical significance, and beyond. In conclusion, we offer an unbiased survey about cannabis to help better weigh its medical value versus the associated risks.
\end{abstract}

Keywords: Addiction, Cannabis sativa/indica, cannabis use disorder, endocannabinoid system, phytocannabinoid synthesis, psychosis, synaptic plasticity, tetrahydrocannabinol.

\section{INTRODUCTION}

Cannabis is a genus of plants indigenous to Asia. Archaeological findings evidence that cannabis was among the first plants cultivated and bred by humans in Asia and later, worldwide. Cannabis was cultivated for its achene fruits (often wrongly referred to as seeds, which are rich in oils and other phytonutrients [1,2], and were used as human food or animal feedstuff) as well as for its fibers, for traditional medicine and spiritual purposes [2-4]. The word cannabis most likely originates from ancient Akkadian (qunnabtu), Neo-Assyrian and Neo-Babylonian (qunnabu), with the meaning of 'way to produce smoke', which was then transformed into kan(n)ab(is) (for instance in Greek) and hanaba, then hennep and hemp in English.

The angiosperm plant belongs to the order Urticales and the family Cannabaceae. However, further taxonomic classification of the Cannabis genus into species, subspecies,

\footnotetext{
*Address correspondence to this author at the Laboratory of Neuromodulation and Metabolism, CNC - Center for Neuroscience and Cell Biology of Coimbra, University of Coimbra, 3004-504 Coimbra, Portugal; Tel: +351-304-502-916; Fax: +351-239-822-776; E-mail: akofalvi@uc.pt

\# This paper is dedicated to Professor E. Sylvester Vizi, M.D., Ph.D., D.Sci., M.HAS., for his Lifetime Achievement, on the occasion of his upcoming birthday.
}

varieties or morphotypes has been a matter of debate for well over two centuries due to (1) its worldwide cultivation and (2) extensive breeding for different purposes by different cultures over several millennia, (3) the presence of escaped and hybridized forms of the different cultivars, as well as (4) several so-called chemovariants (chemotypes) and wild forms, (5) which are, in addition, interfertile [2,5-9]. In general, three Cannabis species are distinguished, namely Cannabis sativa L., C. indica Lam. and C. ruderalis Janisch., corresponding to useful, Indian and wild cannabis plants, respectively. However, others consider only useful cannabis (C. sativa) and the narcotic plant (C. indica) as the only two real species [6], while the most recent and most comprehensive taxonomic work defines Cannabis as one highly variable, highly hybridized and introgressed, panmictic species divided into 6 groups [2].

Genome and transcriptome sequence analyses (e.g., [10-14]) and/or chemotaxonomic analyses (e.g., $[6,7,10]$ ) would further contribute to the better understanding of the biological properties (including sex determination), phenotypic variation, evolutionary history of the genus and the molecular impact of domestication and breeding on the different Cannabis taxa including wild germplasm, landraces, cultivars, chemotypes, strains, biotypes and accessions. 
This dioecious annual flowering plant has taproot system and erect $1-5 \mathrm{~m}$ high shoots $[2,15]$. Female plants are in general more robust but shorter than male plants. The leaves are palmately compound consisting typically of 3-9 (up to 13) linear-lanceolate leaflets with serrate leaf margins [2]. Leaves are arranged in opposite way (decussate) on the basis of the shoots and alternately (spirally) near the shoot tip. The leaflet margins are serrate, and their venation pattern is a peculiar character for morphological identification of cannabis leaves (the veins run out obliquely from the midrib to the tips of the teeth). The abaxial leaf surfaces are pale green and contain white to yellowish brown resinous glands [16]. The male flowers are arranged into loose cymose panicles on male plants, while female flowers are borne on racemes on female plants. However, rarely monoecious individuals bearing both female and male flowers at different shoot parts can be also observed as an intriguing result of human domestification [2]. The flowers are surrounded by greenish tepals often erronously called calyxes. Female flowers, in addition, are encircled by cup-shaped perigonal bracts rich in resin-containing glandular hairs. The female flowers of Cannabis ruderalis and $C$. sativa are small, while those of drug-type hybrids are larger and abundantly covered by secreted material (often termed as resin). The plant is wind-pollinated and produces achene fruits after fertilization. Interestingly, in the absence of pollen grains and pollination, the style and stigma parts of 'virgin' pistils of female cannabis plants bred for their psychoactive compounds are morphologically expanded [17]. Most cannabis varieties are short day plants with the exception of $C$. ruderalis that may be day-neutral and auto-flowering, and grows as weed especially on recently disturbed sites with high soil nitrogen content.

The leaves and floral buds (termed as inflorescences including bracts and tepals rich in resin-producing glandular hairs) of drug-type female cannabis plants are used to produce the recreational drugs (or folk medicine products) called marijuana (also called pot, dagga, ganja, ganza, grass, padinha, and the fruitless flowers referred to as sinsemilla) and additional preparations (e.g., bhang) among others. The scrapped off glandular hairs and their secretory product (resin) itself is called hashish (also known as charas) (see also Section 3.1.2.1). Other extracts including hash oil and wax (known also as shatter, budder or butane hash oil) are also produced from the plants. Its medical use is not only proved by archaeological evidence (including a footed ceramic bowl with carbonized cannabis 'seeds' found in a Bronze Age grave at Gurbaneşti in Romania dated back to 3500 B.C. and similar findings from the Caucasus [18]) but is also extensively documented in written records of several cultures. These include China (in the book "Pen Ts'ao", aka "The Herbal", written by the then Chinese emperor Shen Nung in 2737 B.C.), Egypt (Ebers Papyrus, 1550 B.C.), India (Atharvaveda, 1200-800 B.C.), Greece (Herodotus described in 440 B.C the use by Scythians of the smoke of cannabis 'seeds' for ritual purposes after burial), and the Greek physicians of the Roman Empire (Dioskurides, 100 A.D., Galen, 170 A.D.) [3,8]. The psychotropic properties of Cannabis have also been well documented since long, from around 100 B.C. in China and by Galen in 170 A.D.

After having been used in medicine in the Western world (e.g., in the US since 1840, or even by Queen Victoria of England in 1890), it was listed as dangerous and illicit drug by the Geneva Convention in 1925 and by the Federal Law since 1937. In the last 50 years, an increasingly intense political and scientific debate surrounds the legalization and the safety issues of cannabis-based medical treatments. Vaporized or smoked dried buds, capsules, different extracts applied for instance as oral (oromucosal) sprays (such as Sativex ${ }^{\circledR}$ - known as Nabiximols ${ }^{\circledR}$ in the US), and even prescription drugs including synthetic cannabinoids (synthetic $\Delta^{9}$-tetrahydrocannabinol $\quad\left[\Delta^{9}\right.$-THC $]$ called dronabinol and marketed as Marinol ${ }^{\circledR}$, as well as a $\Delta^{9}$-THCanalog, nabilone - marketed as Cesamet ${ }^{\circledR}$ ) are available in some countries as 'medical cannabis' [19-21] (see also Sections 3.3.3 and 3.3.11).

In spite of ongoing clinical trials with various cannabinoid compounds [21], the medical use of marijuana (e.g., for treating neuropathic pain associated with multiple sclerosis, muscle spasticity, eating disorders, reducing nausea and vomiting in chemotherapy, and alleviating the anorexia associated with AIDS-related weight-loss) is still illegal in most countries with few exceptions (Australia underway, Canada, Cyprus, Chile, Croatia, Czech Republic, Germany, Israel, Italy, Puerto Rico, US). This is mainly due to political and legislative reasons and the fact that the concentrations of the active compounds cannot be controlled within the smoke of cannabis plant. To circumvent this, medical cannabis oils with controlled $\Delta^{9}$-THC:cannabidiol (CBD) (Fig. 1) content are progressively substituting smokable medical marijuana [22,23]. The long-term health consequences of marijuana use (especially under the age of 30) are not clear either: safety concerns also include risk for dependence, psychosis and cognitive problems (see Sections 3.1.2.3-4; 3.3.6-9). For more details about the medical, legal and ethical perspectives as well as future research about medical marijuana or purified cannabinoids the authors are kindly directed towards $[8,19,20,24-26]$, as well as to Section 3.3.3.

Marijuana is estimated to be consumed by up to 227 million people and, thus, represents the most used illicit drug substance in the world [27] (see Section 3.1.1) besides being a highly valuable (although illegal) crop [2]. Consequently, its major psychoactive compound ( $\Delta^{9}$-THC) (Fig. 1) is the fourth most popular and addictive recreational chemical (following caffeine, ethyl alcohol and nicotine) [2]. In this review, we aim at following the long journey of phytocannabinoids from their biosynthesis in the plants towards the receptors in humans where they exert their potential effects.

\section{THE CANNABIS PLANT: ACTIVE COMPOUNDS, IDENTIFICATION AND BIOSYNTHESIS OF PHYTOCANNABINOIDS}

\subsection{Active Compounds of Cannabis Plants with Emphasis on Phytocannabinoids}

Cannabis plants contain more than 545 known compounds [28,29]. In addition to phytocannabinoids, they include alkanes, sugars, nitrogenous compounds (such as spermidine alkaloids or muscarine), flavonoids, noncannabinoid phenols, phenylpropanoids, steroids, fatty acids, approximately 140 different terpenes that are predominantly monoterpenes such as $\beta$-myrcene, $\alpha$ - and $\beta$-pinene, $\alpha$ terpinolene, but also sesquiterpenes including $\beta$ - 
caryophyllene, di- and triterpenes, as well as various common compounds [30-37].

The strong and very characteristic odor of cannabis plants (and drugs, including hashish) is caused predominantly by terpenes. $\beta$-caryophyllene and its oxidation product, caryophyllene oxide are used as leading substances to train dogs to detect hashish and marijuana [3840] (see also Section 3.2.3.3). It has to be noted, however, that many of these terpenes (especially monoterpenes such as $\alpha$-pinene and limonene) are volatile and, therefore, present in higher amounts only in fresh material [41]. In spite of the scarceness of available data on cannabis terpenes and their medicinal effects [37,42-46], two stereospecific monoterpene synthases (limonene synthase and $\alpha$-pinene synthase) were cloned and characterized from cannabis trichome cells [47].

Yet, the most conspicuous and noteworthy compounds of cannabis are called phytocannabinoids. Natural cannabinoids are often termed as phytocannabinoids in order to distinguish them from the chemically different endogenous cannabinoid receptor ligands (endocannabinoids) and from synthetic cannabinoids such as nabilone, dexanabinol and ajulemic acid (Fig. 1). Although in general, phytocannabinoids are considered unique to this genus, substances with phytocannabinoid activity have been found in liverworts (Radula marginata and $R$. perrotteti) [48,49], in the composite Helichrysum umbraculigerum [50] and in several additional plants (see Section 3.2). Phytocannabinoids of the cannabis plant (hereafter referred to as phytocannabinoids for the sake of simplicity) are $\mathrm{C}_{21}$ terpenophenolic compounds with physiological and often psychotogenic effects. They possess monoterpene and alkylresorcinol (i.e., olivetol or olivetolic acid) moieties in their molecules.

More than 109 phytocannabinoids are present in cannabis [28]. These can be classified into several major classes each having a representative molecule such as cannabigerol (CBG), cannabichromene (CBC), CBD (Fig. 1), cannabinol (CBN) and tetrahydrocannabinol (6a,10atrans-6a,7,8,10a-tetrahydro-6,6,9-trimethyl-3-pentyl-6H-

dibenzo[b,d]pyran-1-ol, later abbreviated as $\Delta^{9}$-THC) (Fig. 1) $[30,32]$. These classes contain the different isomers, biogenic precursors (such as $\Delta^{9}$-THC acids $\mathrm{A}$ and B for $\Delta^{9}$ THC, abbreviated hereafter as $\Delta^{9}$-THCA), degradation products and/or artifacts of the representative compounds. For instance $\Delta^{9}$-THC(A) isomers may be present both as cis or trans isomers. In addition, they occur both in 9,10 and 8,9 double-bond configurations referred to as $\Delta^{9}$-THC(A) and $\Delta^{8}$-THC(A), respectively. $\Delta^{8}$-THC is thermodynamically more stable, but at least $20 \%$ less active than $\Delta^{9}$-THC and probably represents an analytical artifact [30]. Alkyl (propyl) homologs of the C-3 $n$-pentyl side chain of the different phytocannabinoids including $\Delta^{9}$-THC, CBC, CBD and CBG are termed as $\Delta^{9}$-tetrahydrocannabivarin $\left(\Delta^{9}\right.$-THCV), cannabichromevarin (CBCV), cannabidivarin (CBDV) and cannabigerovarin (CBGV), respectively. Studies elucidating structure-activity relationships of $\Delta^{9}$-THC and its synthetic analogs identified the C-3 side chain as the key pharmacophore for pharmacological potency and for ligand affinity and selectivity at the cannabinoid receptors [51]. Some of the identified phytocannabinoids such as CBN and cannabicyclol are probably the respective degradation products of $\Delta^{9}$-THC and CBC, formed as a result of enzymatic activity [30,32]. $\Delta^{9}$-THC is oxidized to CBN in the presence of air (oxygen) and UV light [16].

Phytocannabinoids are thought to be synthesized and accumulated primarily in the form of phytocannabinoid acids (e.g., cannabidiolic acid, CBDA; and the above mentioned $\Delta^{9}$-THCA forms) in planta [52-54]. Therefore, the concentrations of phytocannabinoid acids are much higher in fresh plants than those of the respective neutral phytocannabinoids. The latter are formed by non-enzymatic decarboxylation (and thus neutralization) of the phytocannabinoid acids, which occurs predominantly during the processing of the herbal product (drying, storage, heating) and provides the most active compounds (e.g., CBD, $\Delta^{9}$-THC) [55]. $\Delta^{9}$-THC has been first isolated in 1942 [56] but its correct structure assignment was done by Yechiel Gaoni and Raphael Mechoulam in 1964 using nuclear magnetic resonance spectroscopy (NMR) [57]. In addition to its euphoriant psychotropic property, acute or chronice $\Delta^{9}$ THC administration also produces a wide variety of therapeutically relevant peripheral and central effects, both in animal models and man $[2,37,58]$, while $\Delta^{9}$-THCA has mostly antibacterial and antibiotic potential [16]. For the full spectra of the molecular targets and the effects of phytocannabinoids, see Section 3.

Several other phytocannabinoids have been also shown to have medicinal value. For instance, CBD, a nonpsychoactive phytocannabinoid, may account up to $40 \%$ of the cannabis extracts, and was shown to relieve convulsion, inflammation, cough, congestion and nausea, and have anxyolytic, antipsychotic, analgesic, sedative, anti-epileptic, sleep-inducing, neuroprotective, antirheumatic, sebostatic, antibiotic and anticancer (antioxidant) effects, either in animal models or in man or both [2,37,59-62]. CBN exerts sedative, antibiotic, anticonvulsant, anti-inflammatory and mild psychotropic effects [30]. Other phytocannabinoids may also possess therapeutic potential, including

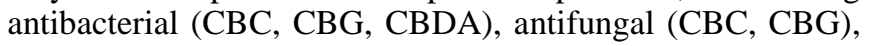
anti-inflammatory (CBC, $\mathrm{CBG}$ ), analgesic or sedative (CBC, CBG, cannabidiolic acid), or even antileishmanial (a recently isolated CBG derivative, 4-acetoxy-2-geranyl-5-hydroxy-3$n$-pentylphenol) effects $[16,30,32,37,63,64]$ (see also Sections 3.3.3-6).

Several data support that CBD interactively and positively modifies the effects of $\Delta^{9}$-THC: the combined use of $\Delta^{9}$-THC and CBD reduces the adverse (e.g., psychotogenic) effects of $\Delta^{9}$-THC while increasing its clinical efficacy and prolonging its duration [2,65]. Furthermore, the effects of extracted phytocannabinoids (applied as single-molecule pharmaceuticals) often differ from that of the crude drugs (marijuana, hashish), which outlines the importance of the highly complex interactions of the natural constituents present in the plant [2]. The abovementioned mouth spray (Sativex ${ }^{\circledR}$ - nabiximols, by GW Pharmaceuticals, London) is the only available licensed medication based on natural phytocannabinoid extracts to treat spasticity in patiens with multiple sclerosis $[21,66]$. Sativex contains approximately equal amounts of $\Delta^{9}$-THC and $\mathrm{CBD}$, which is achieved using identical cannabis clones grown under strictly controlled conditions for extraction, and followed by refining. Sativex has been in three phase III clinical trials as adjunctive treatment to optimized chronic 
opioid therapy in cancer patients who experience inadequate analgesia. In 2015, GW Pharmaceuticals and Otsuka announced that Sativex failed to produce superiority over placebo in the overall cohort involving patients from Mexico, Europe and the US [67]; albeit in the last two trials it did show significant reduction in sleep disruption, as well as superior analgesia in the US cohort [68]. Sativex also scored significant results on a number of secondary endpoints including subject global impression of change and physician global impression of change [68]. Other preparations containing CBD or $\Delta^{9}-\mathrm{THCV}$, or CBD and $\Delta^{9}-$ THC as the main active ingredient(s) are also in phase II and III trials to treat different diseases [21-23].

The phytocannabinoid content and composition is different in female and male plants, and varies during plant development and among the different organs [2,69-71]. For instance, the $\Delta^{9}$-THC content of drug-type cannabis leaves is more or less constant during development, while that of the bracts increases considerably during flowering [72]. Several phytohormones have been shown to influence the biosynthesis of terpenoids and $\Delta^{9}$-THC in cannabis plants [43-46]. In addition, the phytocannabinoid production is influenced by the cultivation protocol used and by environmental factors (stressors) such as humidity (drought), temperature, soil nutrient content [2,15,70,73,74] and illumination conditions (including light quality and quantity, and photoperiod) $[2,69,75]$. Phytoannabinoid content seems to increase under stress conditions, however, as noted by Small [2], it is difficult to estimate this value on whole plant basis as the lowermost leaves with low $\Delta^{9}$-THC content are in general dropped by the plant under such conditions. Stress also causes the plant to grow smaller, but in this case the net change in the overall phytocannabinoid production is not fully understood. The relative ratio of phytocannabinoids vary among the different cannabis species, strains and hybrids, which is most relevant in the case of the $\Delta^{9}$ THC/CBD ratio [2,36,42,72] (see Sections 3.1.1, 3.2.3.3, 3.3.3.1. and 3.3.8 as well). Although not typically, but the phytocannabinoid composition and/or content among different individuals of the populations of the same cultivar may also show considerable variation [76].

Chemotaxonomic evaluation has led to the establishment of three major chemotypes: fiber-type chemovariants contain predominantly CBD, intermediate chemovariants have equal amounts of $\mathrm{CBD}$ and $\Delta^{9}-\mathrm{THC}$, while the latter is the major compound in drug-type plants [2,71,72,77-79]. In spite of quantitative variations observed in phytocannabinoid contents (e.g., [75]), it has been demonstrated that the chemotype is not influenced by sex or developmental age [70]. Due to their different phytocannabinoid composition, the distinct species and/or strains (chemotypes) have different medical and psychotropic potential. Therefore, the genetic background of the different chemotypes $[69,77,80,81]$ as well as their chemotaxonomy especially in terms of molecular/metabolic fingerprinting $[6,7,10,42,82]$ have been extensively studied. Metabolic fingerprinting may be also used in forensic identification of the different chemotypes or during quality control and breeding/selection of the different drug-type cannabis lines [42,70]. In addition, in countries where industrial cultivation of fiber-type hemp is allowed, the $\Delta^{9}$ THC content of cultivars should not exceed the allowed level which normally varies between $0.2-0.35 \% \Delta^{9}$-THC in the inflorescence or young infructescence of plants on a dry mass basis in Europe, Canada and New Zealand [2,83-86]. Considering that $1 \% \Delta^{9}$-THC content is generally accepted as the treshold for the intoxicating potential of marijuana, cultivars with $\Delta^{9}$-THC content (slightly) higher than $0.35 \%$ are also allowed for cultivation in Switzerland and in some parts of Australia [2]. However, the permitted $\Delta^{9}$-THC content of seeds and seed oils of gastronomic purposes is even much lower [2]. On the other hand, several pharmacological firms as well as clandestine breeders breed different drug-type strains with specific phytocannabinoid composition, including strains that selectively overproduce one phytocannabinoid (e.g., $\Delta^{9}$-THC without CBD - aka skunk, CBD, CBC or CBG) at levels over $10 \%$ [2].

The insect-repellent (anti-herbivorous), antimicrobial and antifungal properties of $\Delta^{9}$-THC and other phytocannabinoids and terpenes produced by cannabis are extensively reviewed elsewhere $[15,87]$, and suggest that these compounds may serve for self-defense purposes $[2,15,88]$. However, some data with phytocannabinoid-free Cannabis breeding lines do not confirm this hypothesis [81], while other works have clearly demonstrated that THCA and cannabichromenic acid (CBCA) induce necrotic cell death in leaf cells, and may be, thus, involved in self-defense and senescence [89]. These compounds may be involved in the minor allelopathic properties of Cannabis plants $[2,88]$.

It has been also suggested that - similarly to several phenolic compounds of plants - $\Delta^{9}$-THC may have a role in protecting the plants from high UV-B radiation. This was concluded on the basis of increased $\Delta^{9}$-THC content found in (1) Cannabis plants originating from locations with intense ambient UV-B irradiation [90], (2) in drug-type Cannabis plants exposed to high UV-B light under greenhouse conditions [91], and from the fact that the $\Delta^{9}$-THC of the resin of fiber-type Cannabis did not show photochemical decomposition upon UV and visible light exposure [92]. However, it is important to mention that the $\Delta^{9}$-THC content of fiber-type plants did not vary with increased UV irradiation under the same greenhouse conditions [91], and that the CBD content of the resin of fiber-type plants decreased upon exposure to the same UV and visible light regime [92]. In addition, the phytocannabinoid containing and producing glands are concentrated on the lower (abaxial) side of the leaves and the perigonal bracts in the inflorescence (see Section 2.4), further questioning their speculated protective effect against UV radiation [2]. These data show that a better understanding of the effect of UV light on $\Delta^{9}$-THC biosynthesis and accumulation in different varieties is necessary.

\subsection{Identification of Cannabis and Its Products}

The identification of cannabis plants and products may be done on the basis of observation of general plant morphology, that is, the typical shape and venation of leaves and the structure of the inflorescence. However, this is not always possible in case of forensic samples and different cannabis products. With the exception of hashish oil and other extracts, micromorphological features such as characteristic trichomes on the surface of the fruiting and flowering top may be microscopically identified (see [16,30] and below in Section 2.4). 
Furthermore, phytocannabinoids provide a unique chemical fingerprint for cannabis identification and can be unequivocally identified by several analytical methods $[16,93]$. These include high performance liquid chromatography (HPLC) [10,75,89,94-96], gas-liquid chromatography (GLC) [72,75,97-100] or gas chromatography (GC) $[5,6,28,30,71,72,84]$ coupled with flame ionization or mass spectrometric (MS) detection. In addition to discrimination between the different cannabis species, cultivars, chemovariants and samples collected by forensic scientists on the basis of their phytocannabinoid composition, these data may even provide information about plant cultivation (indoor or outdoor) and about the country of origin [30]. Promising quantification and separation of phytocannabinoids can also be achieved using liquid chromatography coupled with ion trap mass spectrometry [101].

For chromatographic analyses, dried plant material (sometimes prepared previously as dry powder - $[84,94,99]$ ) is usually incubated for approx. 1-4 h with solvent (e.g., petroleum ether - [72], chloroform [5,6,44-46,99], hexane [84,89], methanol - [96], chloroform/methanol [16,28,94,101-103], ethanol - [77], methanol:ethanol (1:1) mixed with equal amount of $\mathrm{CHCl}_{3}$ - [95], or sequentially by various solvents - [64]). The extract is then filtered, sonicated and/or centrifuged, and in some cases, directly injected to the chromatograph [28] or dried under nitrogen stream and then dissolved in another solvent (e.g., in cyclohexane - [72], methanol - [44-46,89], chloroform plus internal standard in methanol - [5], acetone with internal standard - [6], internal standard in ethanol - [99]) before injection. Internal standards such as 4-androstene-3,17-dione (e.g., [5,28,99,102]), anthracene (e.g., [96]), phenantrene (e.g., [77]), n-eicosane [6], chrysene- $d_{12}$ [84] or $\alpha$-colestane (e.g., [72,104]) are used. Sometimes derivatization (silylation or methylation) is necessary to obtain information about phytocannabinoid acids, the dominating phytocannabinoids, but the total phytocannabinoid content can be determined from samples without derivatization $[16,30,64]$. Solid phase-micro extraction methods of Cannabis samples are also available [16].

HPLC (e.g., [75,89,94-96]) or ultra-performance liquid chromatographic (UPLC) [44,45] analyses using carefully optimized gradient elution programs, sometimes reversed phase columns and/or diode-array detection also enable the quantification of the major phytocannabinoids for chemotyping, the evaluation of psychoactive potency, and finally, the quality control of medicinal marijuana.

There are different protocols suitable for rapid screening and on-site field testing, including thin-layer chromatography, color reactions (such as Fast Corinth V salt test, Fast Blue B salt test, Duquenois-Levine test - all described in detail in [16]), macro- and micromorphological (microscopic) observations and a combination of these latter may be also applied [16,30]. Some chemotaxonomic studies used the different phytocannabinoid ( $\Delta^{9}$-THC and CBD) banding patterns on starch gele electrophoresis to discriminate between the different accessions [6,7]. The pros and cons of the use of planar chromatographic methods (such as classical thin layer chromatography, overpressuredlayer chromatography, automated optimal performance laminar chromatography - OPLC, or automated multiple development - AMD) for evaluation of cannabis samples are carefully compared and discussed elsewhere $[16,93,103,105]$.

DNA-based profiling techniques used for cannabis identification are reviewed by others $[16,106]$ and the DNA sequence of some cultivars is already publicly available $[11,12]$. Based on the polymorphisms of the enzyme responsible for $\Delta^{9}$-THCA synthesis [14], fluorescent duplexPCR test [82] and single nucleotide polymorphism assay [83] were developed and successfully applied to discriminate between drug-type and fiber-type cannabis. Recently, a candidate gene involved in phytocannabinoid biosynthesis has been identified that clearly distinguishes these two chemotypes [14] (see later in Section 2.3).

Similarly, ${ }^{1} \mathrm{H}$-nuclear magnetic resonance spectroscopy was recently proved to be a promising non-destructive, fast and sensitive method to identify chemotypes during the entire cultivation period [96]. The extraction methods used for metabolic fingerprinting are discussed elsewhere [42], while data about trichome isolation are discussed in Section 2.4 .

\subsection{Biosynthesis of Phytoannabinoids in the Cannabis Plant}

Phytoannabinoids of the cannabis plant are prenylated polyketides (terpenophenolic compounds) which derive from isoprenoid and fatty acid precursors. However, some molecular details of their biosynthetic pathway still need to be elucidated.

The first committed step of phytocannabinoid biosynthesis is the prenylation of the phenolic moiety (the polyketide-derivative, 5-pentenyl resorcinolic acid, also termed as olivetolic acid, OA) with the terpenoid (isoprenoid) moiety, geranyl pyrophosphate (GPP) (Fig. 2). This condensation of OA and GPP is driven by the soluble geranylpyrophosphate:olivetolate geranyltransferase (GOT) [107], and yields CBG acid (CBGA) which is the direct precursor of CBDA, CBCA and $\Delta^{9}$-THCA. CBGA is converted directly to $\Delta^{9}$-THCA by $\Delta^{9}$-THCA synthase enzyme in all cannabis strains [6,55,80,104,108,109]. The conversion of CBGA to CBDA or to CBCA is driven by CBDA synthase or CBCA synthase, respectively [55,110113]. Both $\Delta^{9}$-THCA synthase [114] and CBDA synthase [113] are FAD-dependent oxidases responsible for the stereospecific oxidative cyclization of the monoterpene moiety of CBGA in the presence of molecular oxygen required for catalysis. The enzymes converting CBGA to different phytocannabinoid acids are thought to be localized in monomeric form in the cytosol $[109,114]$. Additionally, CBGA and $\Delta^{9}$-THCA synthase activity was also demonstrated in the secretory cavity of the glandular trichomes (see Section 2.5) [108].

In addition, the above-mentioned enzymes can also convert CBGV (the propyl homolog of CBG) to the respective propyl homologs $\left(\triangle^{9}\right.$-THCV, CBCV and CBDV) of the major phytocannabinoids $[115,116]$. CBGV is thought to be formed by the condensation of GPP with divarinic acid (5-propyl resorcinolic acid, a $\mathrm{C}_{10}$ compound) instead of OA (a $\mathrm{C}_{12}$ compound) [107,116].

Using deep sequencing technology, the draft genome and transcriptome sequences of a drug-type cannabis strain 
were determined and compared with that of fiber-type hemp cultivars [12]. These data showed that $\Delta^{9}$-THCA synthase excusively occurred in the transcriptome of the drug-type cultivars, and was replaced by CBDA synthase in fiber-type hemp cultivars, explaining the different phytocannabinoid composition of the studied varieties [12]. Recent studies have shown that the presence of a nonfunctional CBDA synthase (positively selected during breeding) distinguishes the drug-type cultivars from fiber-type hemp [14]. This is the first work that provides a tool to clearly dissociate these two chemotypes for the benefit of both agriculture and drug policy [14]. Phylogenetic and genetic analyses suggest that gene duplication and divergence events as well as other genetic factors (quantitative trait loci) have influenced the drug content and potency of the different chemotypes [14]. As discussed in detail elsewhere [2,73,77,117], qualitative and quantitative aspects of phytocannabinoid production (and its inheritance) have to be considered, the former being influenced by the genes involved in phytocannabinoid production, their genetic control and inheritance. The absolute quantity of phytocannabinoids produced by an individual plant is influenced by the growth and development of phytocannabinoid producing structures (i.e., secretory glands and organs containing many of these glands - see Section 2.4). These quantitative aspects are strongly influenced by environmental factors (see Section 2.1), but are independent from biosynthetic pathways and are very probably determined by several - yet mostly unknown genes (quantitative trait loci) [2]. Therefore, $\Delta^{9}$-THC-less cannabis may be bred by disruption of phytocannabinoid biosynthesis or gland development [81]. However, detailed discussion about these genetic factors is beyond the scope of this paper and is discussed elsewhere [2,69,73,77,80,81].

It should be mentioned that the GOT-catalyzed prenylation of OA may also occur with neryl-diphosphate besides with GPP, resulting in the formation of cannabinerolic acid (the trans-isomer of CBGA - [109]), which is subsequently converted into cannabinolic acid [107]. Transcriptomic analyses of glandular hairs identified an expressed sequence tag encoding a predicted prenyltransferase probably corresponding to GOT [11]. Interestingly, the encoded protein related more to members of the membrane-bound chloroplast-localized family of prenyltransferases than to soluble prenyltransferases [11], which may indicate the role of plastids in this reaction. Recently, the gene encoding this aromatic prenyltransferase responsible for the condensation of OA and GPP has been identified [118].

The phenolic moiety (OA) of phytocannabinoids is thought to be formed by the condensation of three molecules of malonyl-CoA and one molecule of n-hexanoyl-CoA into a $\mathrm{C}_{12}$ polyketide, followed by the cyclization of the latter. The first reaction is driven by a putative type III polyketide synthase (termed as tetraketide synthase, TKS - [119], or olivetol synthase - [120]), the presence and activity of which needs further elucidation [11,107,119-124]. The C2-C7 intramolecular aldol condensation with carboxylate retention (the cyclization) is catalyzed by a recently identified polyketide cyclase, OA cyclase (OAC) [119], which enzyme functions in concert with the TKS to generate carbon scaffolds and OA. In case of the synthesis of the propyl homologs, instead of three molecules of malonyl-CoA, only two are condensed with n-hexanoyl-CoA, resulting in a $\mathrm{C}_{10}$ polyketide, which is subsequently cyclisized to DA. A cytoplasm-located acyl-activating enzyme (hexanoyl-CoA synthase) involved in the activation of medium- and longchain fatty acids (including hexanoate) has been identified by transcriptome analysis of the trichomes of female flowers [125]. On the basis of the high transcript abundance of desaturases and lipoxygenases found in these analyses, it has been suggested that hexanoate may be formed through oxygenation and breakdown of unsaturated fatty acids [125]. However, other transcriptomic analyses of RNA isolated from Cannabis glands suggested that hexanoyl-CoA was produced via de novo fatty acid biosynthesis in the plastids [11]. Therefore, the pathway leading to hexanoate formation may need further investigations. Malonyl-CoA is derived from the carboxylation of acetyl-CoA by acetyl-CoA carboxylase [11].

The precursors of GPP are isopentenyl pyrophosphate and dimethylallyl pyrophosphate. These molecules can originate from the cytoplasmic mevalonate pathway or from the plastid-located non-mevalonate pathway (2-C-methyl-Derythritol 4-phosphate/1-deoxy-D-xylulose 5-phosphate pathway). Studies using ${ }^{13}$ C-labeled glucose [126] and analyzing the transcriptome of glandular trichomes $[11,12]$ have proven that the latter is active during phytocannabinoid biosynthesis. The same plastidial biosynthetic pathway is needed for the production of monoterpenes and diterpenes, outlining the active contribution of plastids to the biosynthesis of terpenes and phytocannabinoids, which is also bolstered by the presence of plastids with peculiar ultrastructural features in the secretory cells of the glands (see Section 2.5).

Pure $\Delta^{9}$-THC has an extremely high market price, probably reflecting that both the organic synthesis and the isolation and purification of the different phytocannabinoids from Cannabis plants by different chromatographic methods [89,127-131] are not simple procedures. Therefore, there were several attempts to produce $\Delta^{9}$-THC biotechnologically. $\Delta^{9}$-THCA synthase $[108,114]$ and CBDA synthase [132] have been already cloned and heterologously expressed in transgenic tobacco hairy root cultures [108,114], Spodoptera frugiperda insect cell cultures [114] and transgenic methylotrophic yeast (Pichia pastoris) [132]. These systems produce recombinant enzymes and yielded $\Delta^{9}$-THCA or CBDA when fed with CBGA. Upon heating, smoking or under alkaline conditions, $\Delta^{9}$-THCA and CBDA are non-enzymatically decarboxylated to their respective neutral forms $\left(\Delta^{9}\right.$-THC and CBD) $[53,130]$. Transgenic yeast expressing OAC-TKS was also able to efficiently produce OA in the presence of its precursors [119]. Works with recombinant proteins may contribute to the understanding of the structure-function of the different enzymes (e.g., $\Delta^{9}$ THCA synthase $[108,114,133])$ involved in phytocannabinoid biosynthesis. After the necessary optimization of metabolic engineering, the invested effort may result one day in the establishment of an enzyme-based, high purity, scalable fermentation production system for $\Delta^{9}$ THC or other cannabinoids in microorganisms or other systems. Using combinatorial biosynthesis approaches, these systems may alternatively be also used for the production of novel phytocannabinoid derivatives. Knowledge about the enzymes of the biosynthetic pathway and about the pathways producing its precursors is essential also for molecular breeding of cannabis plants with specific phytocannabinoid 
content (i.e., low- $\Delta^{9}$-THCA and low-CBDA hemp) through targeted mutagenesis.

\subsection{Glandular Trichomes of Cannabis}

The different glandular and non-glandular epidermal structures of cannabis have been extensively studied since their first description by Briosi and Tognini in 1894 and 1897 [134-138]. Numerous unicellular, rigid and curved non-glandular trichomes with a slender, pointed apex can be observed on cannabis [16]. In addition, bear claw-shaped cystolithic trichomes may have calcium carbonate crystals (cystoliths) at their bases. The presence of silica (deposited in the cell walls) and calcium carbonate crystals (formed in the vacuole of the non-glandular, cystolithic trichomes) may be useful to identify marijuana or even its ash residues by light or scanning electron microscopy, the latter occasionally coupled with X-ray microanalyses [137]. The simultaneous presence of bear claw-shaped cystolithic trichomes on the adaxial leaf surface, and slender, non-cystolithic trichomes on the abaxial leaf surface are features used for forensic identification of cannabis [16]. Bear claw-shaped cystolithic trichomes also occur on younger stems, but can be only occasionally observed on perigonal bracts, especially on drug-type plants (Fig. 3A) [139]. Identification can be further assisted by the presence of the specific glandular trichomes discussed below. The number, size and distribution of different trichomes on the central leaflets of the compound leaves may be used to distinguish the major Cannabis taxa (i.e., C. indica, C. ruderalis, C. sativa) even before flowering stage.

Three types of glandular trichomes can be distinguished on cannabis plants [135]: the normal green leaf and stem surfaces (except the hypocotyl and the cotyledons) of the whole plant contain small bulbous and large capitate-sessile glandular hairs, while large capitate-stalked trichomes (Fig. 3A-B) with very high phytocannabinoid content (approximately 20 times higher content than those of capitate-sessile glands) develop predominantly on the floral bracts and bracteoles only after flower initiation $[100,137,138]$. The glands have a flattened-disc like head composed of few to many cells and covered by the secretory product accumulated beneath a cuticular sheath [135] (Fig. 3B).

The full-sized bulbous glands are 25-30 $\mu \mathrm{m}$ high and have a short stalk (stipe) and a head with $20 \mu \mathrm{m}$ diameter [135-137]. These heads contain 1, 2 or 4 secretory cells in a single layer, their stalk is composed of 1 or 2 cells, bearing a 1- or 2-celled base layer [136].

The mature capitate-sessile glands have very short axes consisting of one base and one stalk cell layer, and therefore, appear to be attached directly to the bract surface $[135,136]$. They have a larger, circular to slightly oblong head containing 8-13 secretory cells arranged in a single layer of 40-70 $\mu \mathrm{m}$ diameter. Some authors distinguished two types of capitate-sessile trichomes based on their size: big ones present only in the flowers and smaller ones present also on other plant parts (leaves and stems) [134,137]. The CBCA present in the resin of the capitate-sessile glands shows light bluish autofluorescence under UV excitation in fluorescence microscopes [89].

The development of bulbous and capitate-sessile glands starts early after the initialization of bract development, while capitate-stalked trichomes are the last to mature $[135,136]$. Capitate-sessile glands or capitate-stalked glands are the most conspicuous in young or old bracts, respectively [135]. The latter have a multicellular, 150-200 $\mu \mathrm{m}$ high stalk bearing a globular head (diameter: with 50-100 $\mu \mathrm{m}$ ) attached to a 4-celled stalk and a base, and having so-called abscission zones both at the base and upper stalk cell layers $[135,136]$. The heads of both capitate glands have similar structure, but the diameter of capitate-stalked glands is frequently larger than that of capitate-sessile glands [136]. Recently, it has been shown that the average diameter of capitate-stalked glands of a high- $\Delta^{9}$-THC containing medical cannabis strain is $129 \mu \mathrm{m}$ while that of fiber-type, low- $\Delta^{9}$ THC industrial hemp is $80 \mu \mathrm{m}$, and the mean resin volume of the former was more than four times larger than that of the latter [139].

Bulbous and capitate-sessile glands derive from a single epidermal initial $[136,140]$. The head cells, primary stalk and base layers of the capitate-stalked glands also have epidermal origin, but one part of their multicellular stalks derives secondarily from surrounding sub-epidermal and epidermal cells of the bract and is continuous with the bract mesophyll [136,140] (Fig. 3B). The three types of glandular hairs are hardly distinguishable at their early developmental stages [136]. The development of capitate-stalked hairs is first restricted to the vein ridges, but later they also appear on intervein regions but are rarely observed on bract margins. Their development is discussed in more detail by [136].

Interestingly, glands are restricted to the abaxial side of young leaves, to twigs and most prominently to the outer (abaxial) side of the perigonal bracts, while the inner (adaxial) surface of bracts is devoid of glands and contains only occasionally non-glandular trichomes [2,135]. This specific distribution of glands may suggest their protective role of the young flowers and developing fruits similarly to Humulus [139]. The reproductive shoots of male plants also contain relatively high concentrations of phytocannabinoids [141], and also have lower densities of glandular trichomes [97,137]. These include stalked glands covering the tepal, massively stalked glands present on the filament, and special, very large sessile (or short-stalked) glands observed on the inner surface (grooves) of anthers $[2,97,137]$. Due to the presence of these glands, phytocannabinoids can be also detected on the pollen grains [142], however, as male plants are no drug source, these are less studied and will not be discussed here in detail. The various terpenes, phytocannabinoids and other secreted secondary metabolites (flavonoids and aromatic compounds) accumulate mainly in the capitate-sessile (stalkless) and capitate-stalked glandular trichomes of cannabis [97,98,100,134-136,138,143]. Nevertheless, there is no simple quantitative relationship between gland density and phytocannabinoid concentration of various plant organs of the different chemotypes, and the phytocannabinoid content of individual gland types also showed variation depending on the position and maturity of the gland, and on the developmental stage of the plant and the organ $[99,100,144]$. Still, the human selection of narcotic cannabis strains apparently favored greater gland density and/or size [2,135]. This latter has been confirmed by the presence of extremely large gland heads in recently selected strains with extremely high $\Delta^{9}$-THC level [139]. To influence gland density by preventing seed/fruit development is now a standard practice during the production of 
marijuana [139]. This practice is based on the observation that the perigonal bracts grow considerably after fertilization to cover and protect the (developing) seeds/fruits, but their expansion results in a strong decrease in gland density [139].

The capitate-stalked glandular trichomes have large, round, yellow-orange to brown heads in the high CBD and $\Delta^{9}$-THC accumulating breeding lines, and sparkle under the stereomicroscope [81]. Not surprisingly, the ethanol extracts, smoke and vapor of cannabis are composed of a complex mixture of phytocannabinoids and other terpenoids [145]. Phloroglucinol glucoside (a phenolic compound) has been also detected in stem-exudates originating from nonarticulated laticifers and in glandular trichomes $[138,146]$. The floral leaf surface of cannabis plants is resinous and sticky as a result of the presence of the above-mentioned major secretory products (terpenes and phytocannabinoids). In the absence of the latter compounds (for instance in case of phytocannabinoid-poor breeding lines), the surface of the mature female inflorescence is dry and the trichome heads are small, grey and dull, but contain terpenes (except limonene) [81]. Most pure phytocannabinoid constituents are yellow-orange to brown oils (e.g., [64]).

\subsection{Production and Analysis of the Active Compounds in the Glandular Trichomes}

Phytocannabinoids are deposited in the secretory cavity. This intrawall cavity of the glands is limited by the outer wall of the secretory (disc) cells and covered by cuticle and a portion of the middle layer of the cell wall $[138,140,147]$. Cytochemical studies have shown that cellulases are involved in the formation of the cavity [148].

Ultrastructural analyses of the disc cells revealed the presence of dense cytoplasm, transitory lipid bodies and fibrillar material, few dictyosomes and secretory vesicles derived from them, well-developed endoplasmic reticulum and proplastid-like leucoplast sections with few inner membranes and some electron-dense inclusions at the presecretory stage [140,149]. During the secretory stage, vacuolization of the cells occurs, and mitochondria as well as elongated leucoplasts (also termed as lipoplasts, corresponding in fact to secretory plastids) become abundant. A symplast - i.e., large intercellular cytoplasmic connections, plasmodesmata - develop between the secretory cells of the head [140].

The production of the secretory product is associated with the increased number and structural complexity of mature leucoplasts measuring 1.4-1.6 $\mu \mathrm{m}$ in diameter [140]. Leucoplasts have a dense stroma and develop a paracrystalline body (also called a tubular complex or a reticulate body of a diameter of $0.8 \mu \mathrm{m}) \quad[140,149]$ resembling the prolamellar body of etioplasts of darkgerminated angiospersms (Fig. 4A-C) [150]. Such special membrane arrangement is often observed in secretory tissues of different plants [151-157] or in suspensor cells synthesizing plant hormones including gibberellin [158160]. All these tissues - similarly to cannabis disc cells have active plastidial isoprenoid biosynthesis, necessitating the accumulation of geranyl pyrophosphate and isopentenyl diphosphate intermediates. However, in contrast with etioplasts, the tubular complex of cannabis gradually fills the whole stroma area of the mature plastids of the disc cells [140]. These plastids have a huge internal membrane surface and appear only in the secretory cells of actively synthesizing glands and may be, thus, involved in the production of the secreted material. Although their function in $\Delta^{9}$-THC synthesis is less clear, plastids are probably involved in monoterpene biosynthesis and in providing precursors (GPP or lipids or both) for $\Delta^{9}$-THC synthesis.

Electron transparent material - interpreted as a secretion of probably terpenoid nature - appears in the plastids, and is associated with the tubular complex membranes, the envelope, and seems to be secreted through pores of the envelope to the plastid surface, where it appears as a narrow zone of electron transparent material (Fig. 4B-C) [149]. This material then migrates and accumulates along the cell surface close to the secretory cavity [140,149]. It is then directly extruded through modified regions of the plasma membrane into the cell wall next to the cavity and into the cavity where it forms spherical lipophilic bodies accumulating in vesicle-like bodies in parallel with the enlargement of the secretory cavity (Fig. 4D) [138,140,147,161-163]. These vesicles (and their contents synthesized by the disc cells) are perhaps also engaged in the formation and growth of the cuticular sheath surrounding the secretory cavity [147,162-164]. Microchannels that are presumably associated to the release of volatile secretory compounds are formed in the cuticle after vesicle fusion [161]. It is important to note that stalk (stipe) and base cells of the glands as well as surrounding epidermal cells contain chloroplasts, with starch accumulation observed only in the latter [140]. This phenomenon further outlines the role of secretory plastids in the production of the secondary metabolites of the glands.

Interestingly, $\Delta^{9}$-THCA synthase enzyme activity has been found in the non-cellular secretory cavity of glandular trichomes, indicating that this enzyme may also be secreted out along with other compounds to the cavity, and that maybe the biosynthesis of $\Delta^{9}$-THCA also terminates extracellularly [108]. This statement is further confirmed by (1) RT-PCR analyses showing that $\Delta^{9}$-THCA synthase is expressed predominantly in the disc/secretory cells of the glandular trichomes, (2) data from transgenic tobacco plants expressing $\Delta^{9}$-THCA synthase fused to green fluorescent protein showing fluorescence in the secretory cavity of glandular hairs [108], and (3) studies in insect cultures in which the recombinant $\Delta^{9}$-THCA synthase was also secreted to the medium [114]. In addition, a monoclonal antibodycolloidal gold probe for $\Delta^{9}$-THC localized this phytocannabinoid to the cell wall, the fibrillar matrix and the surface of the vesicles in the secretory cavity, the subcuticular wall and the cuticle of the trichomes $[138,165,166]$. At the same time, only minor amounts of $\Delta^{9}$ THC were occasionally localized to the cytoplasm (and organelles such as plastids) of the disc cells or to epidermal cells in the high pressure cryofixed-cryosubstituted [165] or chemically fixed [166] samples analyzed by TEM. In line with these results, other analytical data showed that nearly all of the phytocannabinoid content of capitate-stalked glands was present in the secretory cavity [98]. The intracellular formation and massive accumulation of $\Delta^{9}$-THC would probably lead to death of the plants as both $\Delta^{9}$-THCA and CBCA are cytotoxic and induce apoptosis in cannabis leaf cell suspension cultures [89]. Therefore, the secretion (and transport) of the synthesized phytocannabinoids (or their precursors), or the enzymes responsible for their 
synthesis into the secretory cavity may be needed to prevent cellular damage [134]. In addition, the extracellular $\Delta^{9}-$ THCA synthesis (the reaction generated by $\Delta^{9}$-THCA synthase) results in hydrogen peroxide production [108], which has general antimicrobial properties, similarly to $\Delta^{9}$ THCA itself [87], and may explain the role of $\Delta^{9}$-THCA in plant defense $[15,55]$. $\Delta^{9}$-THCA also induced apoptosis in insect cells supporting the hypothesis of $\Delta^{9}$-THCA being also involved in plant defense against predators [108]. Extracellularly synthesized $\Delta^{9}$-THC seems to be bound to a cell wall component and transported to the secretory cavity with wall materials released from the disc cell wall during gland maturation [166].

In vitro cell suspension cultures and somatic embryos contain practically negligible amounts of phytocannabinoids [167] and no $\Delta^{9}$-THC synthase, and the biosynthesis of these compounds cannot be induced by either biotic or abiotic elicitors in these systems containing undifferentiated cells [168]. Similarly, phytocannabinoids and olivetol synthase activity were reported to be absent [95] or present at trace levels [125] in roots, leaves, fruits and young plants [95], and in concert with this, phytocannabinoids were absent from calli [70]. For instance, the CBDA content of leaves, stems and roots was 4.8-, 48- and 600-times lower, respectively, than that of the female flowers of a fiber-type hemp cultivar [125]. However, they were also detected in very low amounts in seed oil [1], although in this case, they probably reflect contamination with glands from leaf or bract surfaces during improper processing [2]. In addition to glandular trichomes, laticifers and latex of cannabis plants also showed positive histochemical response to phytocannabinoid indicators (Fast Blue B salt, DuquenoisNegm and Beam tests, and Gibb reaction - see below) [169].

These data - along with other works - indicate that phytocannabinoid synthesis is strongly associated with glandular trichomes and their development, and is thus regulated at developmental and tissue-specific levels [168]. However, while the qualitative phytocannabinoid profile of a strain or clone may be characteristic, the phytocannabinoid contents of the different glands can vary in different organs, in different areas of an organ, but also show seasonal variation, and variation with gland maturation [100].

The above discussed destructive analytic methods used for identification and quantification of phytocannabinoids (GC, GC-MS, HPLC, etc.) do not provide spatial resolution of the different compounds at the (sub)cellular level. Therefore, immuno-localization [138,165,166], submicron confocal microscopy based on label-free coherent antiStokes Raman scattering [170] and fluorescence microscopy (using the bluish autofluorescence of CBCA under UV excitation) [89] can be used for in planta visualization of the phytocannabinoids. In addition, several tests and stains are available for histochemical localization and identification of phytocannabinoids. These include Fast Blue B (FBB) salts ( $\Delta^{9}$-THC and CBD show up in red while CBN presents purple coloration) $[143,169]$, Duquenois-Negm test $\left(\Delta^{9}\right.$-THC and CBN both stain purple) [169], Beam test (red-brown color is observed for $\Delta^{9}$-THC and CBN, purple for CBD) [169], and Gibb reaction (for $\Delta^{9}-\mathrm{THC}, \mathrm{CBD}$ and $\mathrm{CBN}$ : yellow color develops upon addition of 2,6-dibromoquinone-4-chlorimide, and blue-black color is observed upon isopropyl-amine addition) [169]. However, the Duquenois test (more exactly the Duquenois-Levine test) used quite often in (field) tests has been shown to give many false positives, and thus its use for phytocannabinoid identification was questioned [171].

There are several methods to isolate and further analyze the glandular trichomes and their metabolites. The heads of the different capitate glandular trichomes may be removed one by one from the plants using a tungsten needle under the stereomicroscope $[99,100]$. The heads can be then placed directly to a solvent (for instance chloroform) for further analyses [100]. Similarly, the resin contents of the secretory cavity of individual glands may be collected and transferred to solvent, which can be either petroleum ether [97] or chloroform - [98], using a fine needle under stereomicroscope [97] or a siliconized glass micropipette in a micromanipulator under inverted microscope [98], as well as a stretched glass pipette head [108], respectively. Other methods are less laborious and meticulous and may yield higher amounts of the glands. For instance, the glandcontaining plant parts may be shaked with powdered dry ice and sieved [172]. Alternatively, the glandular hairs can be removed from the frozen female inflorescences or flowers by shaking this material through a tea leaf sieve and collecting it in a mortar containing liquid nitrogen [121] or by the Bead Beater method adapted from [173] to cannabis [119,125]. During this process, the trichome-rich surfaces were immersed in ice-cold water, then abraded with teflon rotor in the presence of glass beads and isolation buffer, then filtered and washed twice with different buffers [119]. Alternatively, the trichome-rich surfaces that are immersed in sterile water may be sonicated to remove the glands [89]. Other works added phosphate buffered saline solution to the cannabis bract tissue, then vortexed, sieved and centrifuged the material [11]. Glandular trichomes may also be isolated by blending the plant material in cold sodium acetate buffer and through different filtrations [146]. By choice, trichomes may be isolated by laser microdissection and their phytocannabinoid content may be analyzed by liquid chromatography-mass spectroscopy or cryogenic NMR [134]. Interestingly, these studies detected phytocannabinoids not only in the heads of the capitatesessile and capitate-stalked trichomes, but also in the stalks of the latter, indicating the possibility of a more complex biosynthesis and localization of the metabolites within the glands [134].

\section{THE PSYCHOBIOLOGY AND MOLECULAR MEDICINE OF CANNABIS}

\subsection{Recreational Cannabis}

\subsubsection{Need for Weed - Cannabis Abuse in Numbers}

A cross-national analysis of cumulative (lifetime) substance use revealed that cannabis is the third most popular substance after alcohol and tobacco [174]. The United States (US) and New-Zealand registered the highest rates of lifetime use, which amounted to $42 \%$ for both countries. These numbers qualify cannabis as the most widely used illicit substance worldwide [27,174-177], and cannabis use is constantly escalating among adolescents $[177,178]$. In the city of Porto, Portugal, 14.6\% (356 teenagers) of the surveyed 17-year-old adolescents born in 1990 admitted lifetime use of illicit drugs, and 251 referred to hashish whereas 51 mentioned marijuana as the first 
experimented drug [179]. Among the whole surveyed 2465 teenagers, the most popular drug of abuse was cannabis with the lifetime prevalence of $12.6 \%$, followed by cannabis combined with alcohol (5.5\% lifetime prevalence). The pastyear prevalence for cannabis use was $12.3 \%$ while the pastmonth prevalence was almost the same (12.2\%), indicating that almost all who tried cannabis once $(12.6 \%)$ became a habitual user [179]. In the US, the past-year prevalence of marijuana use increased from $4.1 \%$ to $9.5 \%$, comparing the years 2002-2003 with the years 2012-2013 [180]. In the general population, the prevalence of marijuana/cannabis use disorder (CUD; [181,182]) significantly increased practically doubled - from $1.5 \%$ to $2.9 \%$, although among cannabis users, this value slightly decreased from $35.6 \%$ to $30.6 \%$ by 2012-2013 [180]. The authors speculate that beyond certain strength (as gauged by the $\Delta^{9}$-THC vs. CBD content), cannabis cannot push the prevalence of CUD above $31-36 \%$. In other words, virtually every third user may become habitual abuser or dependent of cannabis. The particular significance of CBD will be discussed extensively below (see e.g., Sections 3.1.2.2, 3.2.3.3, 3.3.3.1. and 3.3.8).

\subsubsection{Cannabis Effects}

\subsubsection{The "Ins and Outs" of Cannabis Getting In and Out} of the Body

Cannabis evokes a plethora of biophysical responses, but all depend on its mode of consumption: what, when and how [183]. The dried leaves and flowering buds of the female cannabis plant are used for the preparation of marijuana. Hashish is a concentrated resin obtained from the female Cannabis plant. Evidently stronger than marijuana, hashish can be ingested via smoking and chewing. Kief is a mass of trichomes collected from the leaves and the flowers, and often serves to make hashish from it. Hashish oil is a concentrated mixture of phytocannabinoids, monoterpenes and sesquiterpenes [184], and its baking in oven increases the transformation of the abundant $\Delta^{9}$-THCA into $\Delta^{9}$-THC, the major psychoactive ingredient. Finally, cannabis "wax" is the most concentrated form of $\Delta^{9}$-THC, containing up to $90 \%$ of this substance along with remaining solvents and pesticides [185].

The modes of consumption also strongly affect the psychobiological responses. Inhalation is the most typical mode of consumption [177], which can be achieved by smoking or "dabbing" the smoldering marijuana. If not submitted to burning, the psychoactive content of the product may be different. Oral ingestion delays the onset of effects by 0.5-2 hours, and the circulating levels of $\Delta^{9}$-THC will be smaller but longer-lasting than when cannabis is smoked or intravenously administered [186,187]. There is a delay in the appearance of subjective "high" after plasma peak $\Delta^{9}$-THC levels [187]. This discrepancy prompts the hypothesis that the delay time is required for the generation of some phytocannabinoid metabolites that have major psychobiological effects at cannabinoid receptors [188,189], or that some phytocannabinoid ligands interfere with endocannabinoid inactivation and in turn, increase endocannabinoid bioavailability [190,191], or simply that the partial agonist $\Delta^{9}$-THC needs time to reach sufficient levels around its central receptors in the highly lipophilic environment. Addressing the pharmacokinetics of $\Delta^{9}$-THC, an early study measured a few $\mathrm{ng} / \mathrm{mL}$ plasma $\Delta^{9}$-THC levels after cannabis consumption [192], which would be equivalent with a plasma concentration of a few nmol/L Since this was published more than 3 decades ago, we can assume that the $\Delta^{9}$-THC content of the tested cannabis preparations was not as high as nowadays. In a recent study, blood samples from Swiss drivers suspected to operate motor vehicles under influence were analyzed by liquid chromatography-tandem mass spectrometry [193]. One of the many interesting findings was that the plasma:blood partition coefficient was 1.7 for phytocannabinoids. The authors measured blood levels of $\Delta^{9}$-THC between 0.7-51 $\mathrm{ng} / \mathrm{mL}$, which corresponds to the concentration range of $\sim 2$ $\sim 160 \mathrm{nM}$. This $\Delta^{9}$-THC concentration range is definitely active at cannabinoid receptors in vitro (see Section 3.3.6). Furthermore, the brain likely passively sequesters $\Delta^{9}$-THC from the blood due to its high lipophilicity, thus central $\Delta^{9}$ THC levels can surpass the circulating levels for a prolonged time.

After a single administration of $\sim 10 \mathrm{mg} \Delta^{9}$-THC in a cigarette or $5 \mathrm{mg} \Delta^{9}$-THC intravenously to young adult male subjects, it was found that $\Delta^{9}$-THC levels rapidly drop below $1 \%$ of the original (which was around $1 / 4$ of the smoken dose) within half a day [194]. However, this study could not establish the half-life of $\Delta^{9}$-THC in the plasma because of its constant re-release from the body tissues into the circulation, thus they supposed that the complete elimination of $\Delta^{9}$-THC from the body requires certainly more than 20 hours, but it may take even a week more [194]. After a cumulative administration of 4 cannabis cigarettes each containing 15 $\mathrm{mg} \Delta^{9}$-THC during two days in chronic marijuana users, the half-life of THC in the circulation increased to at least 4 days [195]. This methodological approach probably more adequately reflects phytocannabinoid excretion in regular chronic marijuana users. The excretion of $\Delta^{9}$-THC metabolites can last for several weeks in frequent heavy users [196,197]. The lasting excretion is due to the absorption of $\Delta^{9}$-THC in the adipose tissue which slowly rereleases the substance in the following days [198]. $\Delta^{9}$-THC together with the rest of the phytocannabinoids is metabolized primarily in the liver, via microsomal cytochrome P450 metabolism [199-201]. THC-COOH (11nor-9-COOH-THC) is the secondary metabolite of $\Delta^{9}$-THC, and its presence in body fluids is considered an incontestable proof of $\Delta^{9}$-THC abuse in forensic sciences [201,202]. Nevertheless, it has been recently discovered that a hair test can produce false positive THC-COOH readouts as reflecting external contamination (e.g., from smoke or cannabis handling), stressing that urine and blood tests should be regarded as reliable proofs of cannabis consumption that occurred in the period of past hours to recent weeks [202]. Very recent cannabis consumption that occurred within one day can be solely determined from the saliva $[203,204]$.

\subsubsection{Short-term Cannabis Effects}

Many factors can influence the acute effect of cannabis in humans, including the $\Delta^{9}$-THC/CBD contents, mode of consumption, consumer's life style, frequency of the use of cannabis and other drugs, and gene polymorphisms. Acute cannabis effects can be subgrouped roughly in two categories: physiological effects (many of which exhibit therapeutic potential) and recreational (psychotomimetic) effects. Acute physiological effects encompass anesthesia, airway relaxation, hypothermia, postural hypotension (albeit 
hypertension if not in standing position), hypolocomotion, increased blood flux in frontal, limbic and paralimbic brain areas, catalepsy, tachycardia of 1.2-1.5-times the resting heart rate, decreased intraocular pressure in glaucoma, antiemesis, and increased insulin release, increase in appetite, thirst, food palatability and in caloric intake [198,205-210].

The acute mental and psychotomimetic outcomes of cannabis intoxication cover the sought effects including euphoria, relaxation, richer sensory experiences and increased sociability, as well as the mostly benign and selflimited side effects and misadventures, such as hallucinations, paranoid and grandiose delusions, conceptual disorganization, feelings of unreality, blunted affect, lack of spontaneity, psychomotor retardation, increased reaction time (contributing to driving incapability), social and emotional withdrawal, altered external and internal perceptions including binocular depth inversion, timedilatation, deficits in attention, verbal fluency and working memory and sometimes increased anxiety, dysphoria, paranoia, and panic attacks $[177,183,211-218]$. Most of these effects coincide with schizophrenia-like positive and negative symptoms [215-218].

Four out of the acute effects, namely hypothermia, hypolocomotion, catalepsy and analgesia (altogether: "the tetrade effects") are the cardinal signatures of cannabimimetics (cannabinoid receptor activators) $[219,220]$. To be more precise, only those cannabimimetics produce tetrade effects which activate the cannabinoid $\mathrm{CB}_{1}$ receptor (see Sections 3.2.2-3). However, most acute effects observed in drug-naïve subjects are blunted in regular cannabis users except subjective effects of 'high', tachycardia and increased serum cortisol in both groups [216]. The consequences of chronic cannabis abuse are discussed in the following paragraphs as well as at the end of this review (Sections 3.3.6-8).

\subsubsection{Cannabis Tolerance and Withdrawal}

Tolerance to cannabis effects can be achieved within days in animal experiments [221-224]. In a pioneering study [221], mice were fed daily with $8 \mathrm{mg} \Delta^{9}$-THC dissolved in milk, for 15 days. On the $16^{\text {th }}$ day, the drug-naïve milk-fed control mice responded with hypothermia - a $1.3{ }^{\circ} \mathrm{C}$ decrease in their body temperature - to a single injection of a low dose $(2 \mathrm{mg} / \mathrm{kg})$ of $\Delta^{9}$-THC, while the tolerant mice showed a $1.0{ }^{\circ} \mathrm{C}$ increase on average in their rectal temperature. During 15 days of treatment, body weight and milk intake were not different between groups. No tolerance developed to acute $\Delta^{9}$-THC-induced immobility on day 16 in the chronic $\Delta^{9}$-THC-group, and bodily metabolism of $\Delta^{9}$ THC also remained unchanged in these animals [221]. Apparently, there are subtle differences between species, because rats chronically injected intraperitoneally with $2 \times 5$ $\mathrm{mg} / \mathrm{kg} \Delta^{9}$-THC daily for 14 days showed tolerance both to immobility and catalepsy, albeit reproduced the hyperthermic effect of $\Delta^{9}$-THC [224]. In these tolerant rats, in vivo electrophysiology revealed that $\Delta^{9}$-THC administration no longer affected nigrostriatal dopaminergic activity, while it still stimulated dopaminergic activity in the "reward area", that is, in the ventral tegmentum after 14 days of treatment [224].
These data bolster the observations that in humans, tolerance is developed to most cognitive, physiological and endocrine effects of cannabis except cannabis-induced "high", which underlies the abuse liability of $\Delta^{9}$-THC $[216,225,226]$. For instance, in frequent chronic marijuana consumers, resting-state time overestimation and underproduction can be detected, but intravenous $\Delta^{9}$-THC $(15-50 \mu \mathrm{g} / \mathrm{kg})$ does not significantly affect time perception beyond the already existing impairments [218,227]. Mild tolerance also develops toward the somnolent effects of higher doses of $\Delta^{9}$-THC [228].

In animal models, $\mathrm{CB}_{1} \mathrm{R}$ maximum binding sites $\left(B_{\max }\right)$ decrease throughout the brain upon 2-week-administration of $\Delta^{9}$-THC and the more potent synthetic cannabimimetic, CP55940 (see Section 3.2.3.1; Fig. 1) while CBD produces no such effect [222]. A recent elegant superresolution microscopy study revealed that $\mathrm{CB}_{1} \mathrm{R}$ density dropped by $74 \%$ in the nerve terminals of perisomatic interneurons of the hippocampus of mice treated twice daily with $10 \mathrm{mg} / \mathrm{kg}$ $\Delta^{9}$-THC for 6.5 days [229]. Full recovery took approximately 6 weeks [229]. During the development of tolerance, systemic and cellular energy metabolism show adaptive responses to constant cannabinoid receptor activation, and synaptic plasticity becomes strongly impaired in the autonomic and the limbic nervous systems. These are congruent with the wide variety of physical and psychical discomforts caused by cannabis withdrawal. Early withdrawal studies in rats were enabled by the availability of the first $\mathrm{CB}_{1} \mathrm{R}$-preferring inverse agonist, SR141716A (rimonabant) ([230]; see Section 3.2.3.1). As we will see later, SR $141716 \mathrm{~A}$ not only abruptly prevents central $\Delta^{9}$-THC effects but also impairs basal (constitutive) $\mathrm{CB}_{1} \mathrm{R}$ activity [231,232], worsening the symptoms beyond those caused by a neutral antagonist (see Section 3.2.3.1). In one of the early studies, 4-day regimens with increasing doses of $\Delta^{9}$-THC were abrupted, or in other cases, were substituted with SR141716A [233]. Although the high dose regimens were way beyond those humans could ever be exposed to, even the lower-dose regimens $(0.5-4 \mathrm{mg} / \mathrm{kg})$ caused physical dependence within just a few days. SR141716A precipitated the following withdrawal symptoms (depending on the $\Delta^{9}$ THC doses used): scratching, rubbing face with paws, licking, wet-dog shakes, arched back and ptosis [223,233]. Rats that received the highest $\Delta^{9}$-THC doses showed additional signs: biting, tongue rolling, retropulsion, head shakes, extended limbs or high stepping, ataxia, myoclonic spasms and front paw treading [233]. Abrupt withdrawal did not cause abstinence signs robust enough to be statistically significant [223,233], and this was probably because the lipophilic $\Delta^{9}$-THC and its metabolites are excreted slowly $[199,201]$, and to some extent, may be retained for longer periods in lipid-rich tissues such as the adipose tissue and the brain. The time-course of $\Delta^{9}$-THC (metabolite) excretion probably allows room for gradual recovery with moderate withdrawal symptoms in those behavioral studies in rodents.

Although the above studies were all carried out in young animals, apparently aging strongly influences $\mathrm{CB}_{1} \mathrm{R}$ functioning and its sensibility to chronic agonist exposure. Presynaptic $\mathrm{CB}_{1} \mathrm{R}$ density is already $10 \%$ smaller by 6 months of age in the rat hippocampus, which decreases by the end of the $2^{\text {nd }}$ year to $70 \%$ of the original value measured at 2 months of age [234]. In mouse hippocampal membranes, even greater decrease was observed both in terms of basal 
and CP55940-stimulated [ $\left[{ }^{35} \mathrm{~S}\right]$-guanosine 5'-O-[gammathio]triphosphate $\left(\left[{ }^{35} \mathrm{~S}\right]-\mathrm{GTP} \gamma \mathrm{S}\right)$ binding in the middle-aged (12-month-old) mice (for the explanation of the technique, see Section 3.2.3.1) [235]. Importantly, 8 injections of $\Delta^{9}$ THC during 5 days caused desensitization in $\mathrm{CB}_{1} \mathrm{R}$-coupling, and also reduced hypomotility in response to acute $\Delta^{9}$-THC injection in behavioral assays only in the young (6-8-weekold) mice, rather than in their middle-aged counterparts [235].

There is an increasing demand from CUD patients for the treatment of cannabis dependence [236], but this meets a paucity of available therapeutic tools that could provide some degree of relief $[237,238]$. In $\Delta^{9}$-THC-tolerant rats, classical approaches such as dopamine $D_{1}$ or $D_{2}$ receptor $\left(D_{1} R, \quad D_{2} R\right)$ blockade failed to affect SR141716Aprecipitated withdrawal, although vertical activity and grooming were reduced. In contrast, $\mathrm{D}_{1} \mathrm{R}$ or $\mathrm{D}_{2} \mathrm{R}$ agonists worsened the withdrawal symptoms and triggered seizures [239]. N-acetylcysteine therapy proved so far one of the most useful approaches in a limited number of human patients, and the outcomes of a recently completed clinical trial on the efficacy of $1200 \mathrm{mg} \mathrm{N}$-acetylcysteine on the recovery from cannabis dependence in about 300 CUD patients will be soon published [240].

\subsubsection{Long-term Consequences of Cannabis Abuse}

Long-term marijuana consumption is clearly associated with a risk of addiction [177]. The level of risk is dependent on genetic predisposition, lifestyle, frequency of use and more importantly, the age when frequent marijuana consumption starts. For instance, in the general population, the overall risk of becoming addicted to marijuana is $9 \%$, but the risk almost doubles in adolescents who try marijuana, and is $25-50 \%$ in daily users [177].

There are important gender-dependent differences in CUD: males have higher prevalence for CUD and more frequently seek CUD treatment, while females easier and faster develop cannabis-dependence after initial use [241244]. This predicts that the subjective effects of $\Delta^{9}$-THC are different in the two sexes, and indeed, this is what has been observed: female participants of a recent study were more sensitive than males to the subjective effects of an orally administered low-dose of $\Delta^{9}$-THC [245].

Besides dependence, tolerance and withdrawal symptoms which collectively fall under the term of CUD, long-term cannabis abuse can provoke several alterations in the human body, and only a few of them are benign. Once again, it is difficult to draw a clear picture because of several influencing factors including e.g., individual differences in frequency of use, dosage, life-style and genetic background. Due to the high density and significance of $\mathrm{CB}_{1} \mathrm{Rs}$ in both the developing and the adult nervous tissues, long-term exposure to $\Delta^{9}$-THC and synthetic cannabinoids either preor post-natally has deleterious effects on the fetal, adolescent and adult brain, with permanent neurodevelopmental alterations and serious risks to neuropsychiatric disorders including schizophrenia (see Sections 3.3.7-8).

Chronic marijuana consumption has long-term peripheral effects as well. To start with the toxicity of the smoke, a study ordered by the Canadian government in 2007 revealed that marijuana smoke contains 20-times more ammonia, 3-5-times more hydrogen cyanide, nitrous oxides and some aromatic amines, and 50-70\% more carcinogenic hydrocarbons than the smoke of filter tobacco cigarettes [246]. Consequently, some studies find association between cannabis use and the narrowing and extensive injury of the airways as well as cancers in the respiratory tract and reproductive organs. However, there is no change in the frequency of head-neck cancers in lifetime users when compared to non-users [247-250].

Due to the profound effects of cannabis constituents on the blood vessels and the heart, prolonged use of cannabis alone can provoke serious and sometimes fatal cardiovascular complications in some individuals, even in the absence of comorbid cadiovascular diseases. There are several reports on cannabis-induced multifocal intracranial vasoconstriction, (recurrent) ischemic strokes, cerebellar infarction [251-254], and multiple cardiovascular fatalities [255]. A case of cannabis-provoked recurrent pancreatitis [256], as well as synthetic cannabis- (K2) induced myocardial ischemia in a series of pediatric patients [257] have been also reported.

Chronic cannabis consumption also can have positive outcomes on certain metabolic parameters. Albeit heavy and long-term (15-year) marijuana abuse is associated with 23\% higher caloric intake and 3-times greater alcohol intake as well as increased tobacco use in 3617 young adults in 4 major metropolitan areas of the US [210], some metabolic parameters improve against all odds: A recent study found that current cannabis use in the past 30 days was associated with $16 \%$ lower fasting insulin and $3.7 \%$ lower plasma glucose levels, $1.63 \mathrm{mg} / \mathrm{dL}$ higher high-density lipoprotein-C levels, better insulin sensitivity and smaller waist circumferences compared to those who never used cannabis, among the enrolled 4657 US adults [258]. These positive associations appeared to be short-lived as they were already blunted in those who ingested cannabis more than a month before the survey [258]. Another similar study in the US population $(n=10896)$ found that current and past cannabis users were less prone to diabetes mellitus and had higher HDL levels [259]. This study also reported reduced low-density lipoprotein (LDL, bad cholesterol) and lower triglyceride, glucose and $\mathrm{C}$ reactive protein levels in the serum of past and current users [259]. However, crosscorrelative analysis of the reports also reveals a few discrepancies: increase [210], no change [258] or even a decrease [259] in circulating triglyceride levels were all reported. Also, systolic blood pressure was found to be unaffected among cannabis users in one study [258] while it was found increased by another survey [210]. Nevertheless, multivariate analysis revealed that increased alcohol consumption among cannabis users was the confounding factor contributing to the greater triglyceride and systolic blood pressure values in the above study [210].

In conclusion, past and present cannabis use is associated with a decreased prevalence for metabolic disorders if alcohol use remains low. However, it remains to be elucidated whether chronic cannabis consumption acts as an activator or rather, as a deactivator of cannabinoid receptors in the human body. This question has been prompted by several lines of observations discussed later in this review. One of these observations is truly amazing, because similarly to long-term cannabis use, the $C_{1} R$ blocker SR141716A (rimonabant) also confers beneficial 
effects on the cardiometabolic risk factors, including waist circumference, lipid levels, glycemia and insulin sensitivity [260-263].

\subsection{The Pharmacology and Molecular Biology of the Endocannabinoid System Sensu Lato}

The history of scientific research on cannabis stretches back over 150 years, which has been reviewed elsewhere [264]. The curiosity about the cellular targets of $\Delta^{9}$-THC and related phytocannabinoids has led to the discovery of a great number of proteins and lipid molecules that can be abridged as the "endocannabinoid system sensu lato" (Fig. 5). The term "endocannabinoid" [265,266] was coined to distinguish cannabinoid molecules endogenously produced by the body from those of exogenous origin such as $\Delta^{9}$-THC.

Strictly speaking, the term "endocannabinoid system" refers to the endocannabinoid molecules - among them the most studied ones are $N$-arachidonoylethanolamine (anandamide) and sn-2-arachidonoylglycerol (2-AG); their synthesizing and metabolizing enzymes; the cannabinoid $\mathrm{CB}_{1}$ and $\mathrm{CB}_{2}$ receptors $\left(\mathrm{CB}_{1} \mathrm{Rs}\right.$ and $\left.\mathrm{CB}_{2} \mathrm{Rs}\right)$; furthermore, an elusive endocannabinoid membrane transporter (EMT); and finally, the $\mathrm{CB}_{1} \mathrm{R}$ interacting protein 1a (CRIP1a) which is emerging as a regulator of $\mathrm{CB}_{1} \mathrm{R}$ signaling. However, to tell the endocannabinoid signalosome apart from other signaling families is as difficult as to define the contours of the green color in a rainbow. To fully appreciate the acute and longterm physiological consequences of cannabis intake as well as the therapeutic potential of marijuana-based medicines, it is necessary to overview additional targets which subserve the physiological actions of marijuana. Many phytocannabinoids of the cannabis and other plants [37,267270 ] serve as ligands for receptors beyond the $\mathrm{CB}_{1} \mathrm{R}$ and the $\mathrm{CB}_{2} \mathrm{R}$. Such additional receptors are the serotonin $5-\mathrm{HT}_{1 \mathrm{~A}}$ receptor $\left(5-\mathrm{HT}_{1 \mathrm{~A}} \mathrm{R}\right)$ and the deorphanized GPR55. Phytocannabinoids also interact with ionotropic receptors of the transient receptor potential (TRP) family including the $\mathrm{TRPV}_{1}$ receptor, which is in fact a bona fide ionotropic endocannabinoid receptor (besides being much more). Last but not least, cannabinoids can go nuclear. The psychoactive nature of cannabis ingredients prompts us to focus on the central nervous system, but the role of the endocannabinoid system in some other selected peripheral mechanisms will be also reviewed.

\subsubsection{Endocannabinoids}

Shortly after the discovery and cloning of the $\mathrm{CB}_{1} \mathrm{R}$ [271-273], their first endocannabinoid ligand, $N$ arachidonoylethanolamine (anandamide) was reported $[274,275]$, and its calcium-dependent formation, release and inactivation were characterized in cultured neurons [265]. Following the discovery of the second cannabinoid receptor, the $\mathrm{CB}_{2} \mathrm{R}$ [276], another endocannabinoid, sn-2arachidonoylglycerol (2-AG) was identified [277,278]. Anandamide is at least two orders of magnitude less abundant in the brain than 2-AG [279]. Moreover, anandamide in many assays appeared less efficacious at the $\mathrm{CB}_{1} \mathrm{R}$ and $\mathrm{CB}_{2} \mathrm{R}$ than 2-AG, and is also less potent than $\Delta^{9}$ THC $[219,280-284]$. Consequently, the $\mathrm{CB}_{1} \mathrm{R}$ and the $\mathrm{CB}_{2} \mathrm{R}$ were recognized as 2-AG receptors [283-284], while anandamide together with $\Delta^{9}$-THC fall in the category of partial agonists. Additional endogenous fatty acid-derivative ligands of the $C_{1} R$ and the $C_{2} R$ were also reported (see
Section 3.2.3.4), but more research is needed to better understand their physiological significance. Finally, the endogenous ligands of the "unofficial" cannabinoid receptors GPR18 and GPR55 will be discussed together with their cognate receptors.

\subsubsection{Endocannabinoid Synthesis}

Precursors for both anandamide and 2-AG are believed to be stored in the cell membranes where the synthesis and the release of the two endocannabinoids occur [285,286]. Multiple synthetic routes have been identified for anandamide [286,287]. Its principal precursor is $N$ arachidonoyl-phosphatidylethanolamine (NAPE) [286-288]. Different enzymatic steps can lead to "on demand" anandamide cleavage from NAPE, which can involve the $\mathrm{Ca}^{2+}$-dependent activation of specific phospholipases $\mathrm{A}_{2}, \mathrm{C}$, $\mathrm{D}$ and $\alpha, \beta$ domain serine hydrolase 4 (ABDH4) [286-288]. There is evidence that NAPE-hydrolyzing phospholipase D (NAPE-PLD) is predominantly presynaptic in the hippocampus in excitatory afferents, with highest density in the $\mathrm{CB}_{1} \mathrm{R}$-negative mossy fiber terminals [289]. In the ventral pallidum, NAPE-PLD is also predominantly presynaptic in both inhibitory and excitatory terminals which are often $\mathrm{CB}_{1} \mathrm{R}$-negative, apposing to $\mathrm{CB}_{1} \mathrm{R}$-positive nerve terminals [290]. As only $12 \%$ of NAPE-PLD immunoreactivity appeared dendritic, it is to conclude that NAPE-PLD-mediated anandamide signaling follows the classical pattern as being anterograde, unlike 2-AG signaling, which is principally retrograde at central synapses (see Section 3.3.2).

The chief biosynthetic pathway for 2-AG (when participating in endocannabinoid signaling) starts with the phospholipase-C $\beta$ (PLC $\beta$ )-mediated hydrolysis of phosphatidylinositol 4,5-bisphosphate $\left(\mathrm{PIP}_{2}\right)$ [291] resulting in the intermediate sn-2-diacylglycerol (DAG), from which 2-AG is cleaved by the enzyme sn-2-DAG lipase $\alpha$ $(\mathrm{DAGL} \alpha)$ [292-294]. Additionally, at least two other routes of 2-AG synthesis have been identified [295,296] with elusive physiological importance.

In the nervous tissue, 2-AG - when participating in endocannabinoid signaling - is also thought to be released "on-demand" [297]. This means that typically in dendrites, a depolarization-induced $\mathrm{Ca}^{2+}$ entry or the stimulation of $\mathrm{G}_{\mathrm{q} / 11}$ protein-coupled metabotropic receptors or both activate DAGL $\alpha$ to produce 2-AG in a millisecond to second scale [286,298,299] (Fig. 6). As a footnote, this mechanism is not restricted to neurons: $G_{q / 11}$-coupled receptor activation can trigger DAGL-mediated 2-AG release and consequent $\mathrm{CB}_{1} \mathrm{R}$ activation in transfected Chinese hamster ovary (CHO) cells, too [300]. Some hypothesized that in special cases 2-AG could be pre-synthesized and stored until stimulus [301,302], but till now the theory of "on demand" endocannabinoid release remains the leading concept [303].

\subsubsection{Endocannabinoid Degradation and Transport}

Several enzymes can degrade endocannabinoids, but only a few of them are responsible for the bulk metabolism. Anandamide is hydrolyzed into arachidonic acid and ethanolamine by intracellular enzymes among which the fatty acid aminohydrolase-1 (FAAH-1) bears major importance [304]. FAAH-2 is another enzyme sharing 20\% similarity in its amino acid sequence with FAAH-1, and is expressed in humans but not in laboratory rodents [305], 
which makes difficult to dissect its physiological importance. FAAH-1 is recognized as a therapeutic target in a number of health conditions [306-310], because its blockade increases anandamide tone that can indirectly activate cannabinoid receptors without psychoactive side effects. A third enzyme, a splice variant product of the FAAH-1 gene, the so-called FAAH-like anandamide transporter (FLAT) was described as a protein involved in the transport of anandamide through the cell membranes [311], but it has been "downgraded" later as just one more cytosolic anandamide degrading enzyme [312].

Figure 6 summarizes the major components and steps of 2-AG signaling in a glutamatergic synapse. As for the termination of 2-AG signaling, the presynaptic intracellular monoacylglycerol lipase (MAGL) [313,314] provides the predominant 2-AG hydrolytic activity in the mammalian brain, producing arachidonic acid and glycerol from 2-AG. Acute MAGL inhibition increases 2-AG half-life in the synapse [315-317]. As a consequence of 2-AG being abundant and a full $\mathrm{CB}_{1} \mathrm{R}$ agonist, chronic MAGL inhibition or the genetic deletion of MAGL causes prolonged elevation in 2-AG levels, causing the desensitization of $C_{1} R$ signaling [318-320]. Later, a microglial MAGL-like activity was also reported, but to our knowledge, this MAGL-2 has not been fully characterized [321].

Two additional classes of metabolizing enzymes, $\alpha, \beta$ serine hydrolase domain 6 and 12 (ABDH6/12) are mainly responsible for the remaining 2-AG metabolizing activity [322-324]. In the synapse, these two enzymes are found in the post-synaptic membranes. While ABDH12 faces the lumen or the extracellular space, ABDH6 is rather located in the membrane facing the cytoplasm, thus they can mitigate 2-AG levels at both sides of the membrane [322-324]. Other enzymes including cyclooxygenase-2 (COX-2) [325-328], 12-lipoxygenase [329], 15-lipoxygenase [325] and neuropathy target esterase [330] were also shown to readily metabolize endocannabinoids into bioactive nonendocannabinoid molecules, but these pathways probably gain more importance either in peripheral tissues or under pathological conditions.

The most elusive member of the endocannabinoid signalosome is the purported EMT, which has not yet been identified despite huge efforts, and perhaps it does not even exist - as predicted by models of passive endocannabinoid diffusion driven by hydrolysis [312,331,332] (Fig. 6). Finally, the oddball of intercellular anandamide signaling indeed involves "odd balls": microvesicles. Microglia which can be regarded as the fourth component of the quadripartite synapse [333] - can release membrane segments containing anandamide in the form of microvesicles onto GABAergic synapses with functional implications [334]. This form of endocannabinoid signaling presumably does not require the direct release of anandamide from the membrane into the extracellular space.

\subsection{2. $C B_{1}$ and $C B_{2}$ Cannabinoid Receptors}

The cloning and initial characterization of the central cannabinoid receptor, the $\mathrm{CB}_{1} \mathrm{R}$ were reported in the beginning of the nineties [271-273,335]. Only a few years later, another cannabinoid receptor type, the human and the rat $\mathrm{CB}_{2} \mathrm{R}$ was discovered [276,336,337], with almost exclusive expression in the immune tissues [338]. Both of these two receptors belong to the rhodopsin-like $\mathrm{G}$ proteincoupled receptor (GPCR) superfamily, and contain $7 \alpha$ helical transmembrane domains.

The high similarity in amino acid sequence of the 473 amino acid-long rat $\mathrm{CB}_{1} \mathrm{R}$ [273] and the 472 amino acid-long human $\mathrm{CB}_{1} \mathrm{R} \quad\left(\mathrm{hCB}_{1} \mathrm{R}\right)$ [272] represents evolutionary conservation [339]. One shorter splice variant of the rat and two of the human $\mathrm{CB}_{1} \mathrm{R}$ have also been identified [340,341]. The "normal length" $C_{1} R$ and its expressed splice variants, $\mathrm{CB}_{1} \mathrm{R}_{\mathrm{A}}$ and $\mathrm{CB}_{1} \mathrm{R}_{\mathrm{B}}$ proteins all significantly differ in their pharmacological profile both in terms of the affinity and the efficacy of ligands [282,341]. For instance at the $\mathrm{CB}_{1} \mathrm{R}_{\mathrm{A}}$ and $\mathrm{CB}_{1} \mathrm{R}_{\mathrm{B}}$, 2-AG behaves as an inverse agonist (i.e., it inhibits GTP $\gamma \mathrm{S}$ binding - see Section 3.2.3.1) while anandamide loses efficacy [341]. The $\mathrm{CB}_{1} \mathrm{R}$ in its homomeric form preferentially couples to $\mathrm{G}_{\mathrm{i} / \mathrm{o}}$ proteins [342,343].

The first cloned $\mathrm{hCB}_{2} \mathrm{R}$ has only 360 amino acids, sharing only $44 \%$ overall homology with the $\mathrm{hCB}_{1} \mathrm{R}$, and $68 \%$ identical amino acid identity considering only the 7 transmembrane domains [276]. Mice have $2\left(\mathrm{CB}_{2} \mathrm{R}_{\mathrm{A}, \mathrm{B}}\right)$ while rats have $4 \quad \mathrm{CB}_{2} \mathrm{R}$ splice variant products $\left(\mathrm{CB}_{2} \mathrm{R}_{\mathrm{A}-\mathrm{D}}\right)$ [344,345], with possibly differing pharmacological profile. The $\mathrm{CB}_{2} \mathrm{R}$ also couples predominantly to $\mathrm{G}_{\mathrm{i} / \mathrm{o}}$ proteins [346,347] to inhibit adenylyl cyclase activity, which is thought to be the underlying mechanism of $\mathrm{CB}_{2} \mathrm{R}$-mediated downregulation of immune functions [280,336,348,349].

Both the $\mathrm{CB}_{1}$ (Fig. 7) and the $\mathrm{CB}_{2}$ receptors couple to multiple intracellular effectors, including adenylyl cyclase, extracellular regulated kinase 1/2 (ERK1/2), p38 mitogenactivated protein kinase (MAPK), c-Jun N-terminal kinase (JNK), and phosphoinositide 3-kinase $\left(\mathrm{PI}_{3} \mathrm{~K}\right) /$ protein kinase $\mathrm{B}$ (Akt) [350-359]. Common $\mathrm{CB}_{1} \mathrm{R}$ signaling routes through $\mathrm{G}_{\mathrm{i} / \mathrm{o}}$ proteins are displayed in Fig. 7. For additional signaling pathways, see Guzmán et al. [360] and Harkany et al. [361]. Nevertheless, cannabinoid receptor mediated signaling cannot be viewed mechanistically, as context-dependent decisions are made at signaling crossroads. For example, $\mathrm{CB}_{1} \mathrm{R}$ activation converging onto $\mathrm{PI}_{3} \mathrm{~K}$ and ERK activation promotes survival against ceramide-induced apoptosis [355], but chronic $\mathrm{CB}_{1} \mathrm{R}$ and $\mathrm{CB}_{2} \mathrm{R}$ activation itself stimulates the generation of ceramide, which causes sustained ERK activation via RAF proto-oncogene serine/threonine-protein kinase (Raf-1), leading to apoptosis [360,362,363].

$\mathrm{CB}_{1} \mathrm{Rs}$ and $\mathrm{CB}_{2} \mathrm{Rs}$ can modulate intracellular cation levels. $\mathrm{CB}_{1}$ Rs are negatively coupled to $\mathrm{N}-, \mathrm{L}-, \mathrm{P}-$ and $\mathrm{Q}-$ types of voltage-gated $\mathrm{Ca}^{2+}$ channels (VGCCs) via the $\beta \gamma$ subunit of the $G$ protein, and positively associated with inwardly rectifying $\mathrm{K}^{+}$channels (GIRKs), probably via direct physical complexing [364-369]. As discussed above, under special circumstances, $\mathrm{CB}_{1}$ Rs can trigger intracellular $\mathrm{Ca}^{2+}$ transients, too [370,371]. The stimulation of $\mathrm{CB}_{2} \mathrm{Rs}$ may also produce transient increases in intracellular $\mathrm{Ca}^{2+}$ concentration $\left(\left[\mathrm{Ca}^{2+}\right]_{\mathrm{i}}\right)$ via PLC $\beta$ [284,372], at least in transfected cells. Although initially it was found that the $\mathrm{CB}_{2} \mathrm{R}$ was unable to modulate VGCCs [365,373], later it turned out that those negative data were the consequence of functional selectivity of the ligands (see Section 3.2.3.1) and strain differences [374,375]. Namely, the $\Delta^{9}$-THC analog CP55940 (Fig. 1) inhibited VGCCs in human embryonic kidney cell line 293 (HEK293) transfected with either the mouse or the rat $\mathrm{CB}_{2} \mathrm{R}$ of the long isoform, while the 
aminoalkylindole WIN55212-2 inhibited VGCCs currents only in cells transfected with the $\mathrm{rCB}_{2} \mathrm{R}$ [376].

\subsubsection{Pharmacology of the $C B_{1} R$ and the $C B_{2} R$}

Cannabinoids can be divided into major groups based on their chemical structure. The phenylterpenoid $\Delta^{9}$-THC and all its derivatives which have a pyran ring belong to the first group understandably termed as classical cannabinoids. $\Delta^{9}$-THC-derivatives without a pyran ring are called nonclassical cannabinoids. Some distant phytocannabinoidderivatives are termed as atypical cannabinoids. Other synthetic cannabinoids fall in the category of aminoalkylindoles: anecdotally, the pharmaceutical company Sterling-Winthrop back in the eighties aimed at developing a novel class of COX inhibitors based on the aminoalkylindole backbone, and the first such promising molecule, pravadoline indeed exerted a very strong analgesic and antiinflammatory activity. Soon, these effects were attributed to an off-target cannabimimetic action, and pravadoline research took a new course.

The agonist profile of $\mathrm{CB}_{1}$ and $\mathrm{CB}_{2}$ receptors is considerably overlapping: the ultrapotent classical cannabimimetic HU210 as well as the above mentioned CP55940 (synthesized by Pfizer in 1974) (Fig. 1), $\Delta^{9}$-THC [57] (Fig. 1), WIN55212-2 [377-379] (Fig. 1), anandamide and 2-AG (Fig. 1) are the cannabimimetics most typically used for studying the role of the $\mathrm{CB}_{1} \mathrm{R}$ and the $\mathrm{CB}_{2} \mathrm{R}$, and these ligands do not show significant preference toward one or the other receptor [380,381]. However, many additional endocannabinoids, non-psychoactive phytocannabinoids and novel synthetic ligands differentiate between the two receptors (see Sections 3.2.3.3-4). Based on their contextdependent action, they can be classified as orthosteric ligands such as biased agonists, inverse agonists and neutral antagonists as well as allosteric ligands.

\subsubsection{Multifaceted Interaction of Ligands and G Proteins} with the $C B_{1} R$ and the $C B_{2} R$

As discussed earlier, most GPCR ligands have their own intrinsic efficacy in a given system [382], because when structurally different ligands bind to their cognate receptor they are prone to trigger dissimilar conformational changes. Hence the term "biased agonism" was coined [383]. In other sources, biased agonism is substituted with "agonist-directed trafficking of response" (ADTR) [384,385]. However, we here prefer the term "functional selectivity of the ligands" because biased antagonism is also an existing phenomenon, and curiously, agonists can also act as antagonists at the same receptor under special circumstances. The existence of splice variants for the $\mathrm{CB}_{1} \mathrm{R}$ and the $\mathrm{CB}_{2} \mathrm{R}$ can also greatly increase the odds of odd cannabinoid pharmacology in $e x$ vivo models or cell culture.

The first example for functional selectivity was seen at the level of $G$ proteins in N18TG2 neuroblastoma membranes [386]: WIN55212-2 (up to $100 \mathrm{nM}$ ) was capable of fully dissociating $G_{\alpha i 1}, G_{\alpha i 2}$ and $G_{\alpha i 3}$ from the $C_{1} R$, while the cannabimimetic desacetyllevonantradol $(1 \mu \mathrm{M})$ [387] fully dissociated the $C_{1} R-G_{\alpha i 2}$ and partially disrupted the $\mathrm{CB}_{1} \mathrm{R}-\mathrm{G}_{\text {ail }}$ complex, and finally, the anandamide analog, $R$ methanandamide $(100 \mathrm{nM})$ triggered the dissociation of only $\mathrm{G}_{\text {ai3 }}$ [386]. This also explains why WIN55212-2 has such a high intrinsic efficacy throughout different assays (e.g., [388]), therefore this aminoalkylindole agonist is a popular cannabimimetic tool for research, because it guarantees the success of most functional assays with $\mathrm{CB}_{1}$ and $\mathrm{CB}_{2}$ receptors. An important note for the field is that above $1 \mu \mathrm{M}$, WIN55212-2 stimulates $\left[{ }^{35} \mathrm{~S}\right]-\mathrm{GTP} \gamma \mathrm{S}$ binding in brain membranes of the $\mathrm{CB}_{1} \mathrm{R}-/$ - mice [389], and consequently, it can inhibit glutamate release independently from $\mathrm{CB}_{1} \mathrm{Rs}$ [390,391].

Many GPCRs exhibit a so-called constitutive activity, meaning that they dissociate from GDP-bound G proteins at a low rate either because of the presence of residual levels of endogenous ligands or by a thermodynamically allowed lowprobability reversal of association, in the absence of agonists $[383,386]$. Theoretically, antagonists are not supposed to affect the constitutive auto-uncoupling. An antagonist is called silent or neutral when it only prevents an agonist from activating the receptor, but in itself does not disturb significantly the conformational state of the receptor, hence producing no effect per se [392]. The classical cannabinoid O-2050 [393] and the diarylpyrazole NESS0327 [394] fall under this category as both prevent $\mathrm{CB}_{1} \mathrm{R}$ activation when agonists are present, but per se they do not produce any effect [395]. Notwithstanding, a recent characterization of O2050 revealed some unexpected in vitro partial agonist activity in cyclic AMP assay, and a lack of antagonism on in vivo effects of $\Delta^{9}$-THC and WIN55212-2 [393]. In our hands, O-2050 $(1 \mu \mathrm{M})$ had no effect on the depolarizationinduced release of GABA, glutamate and serotonin in the frontal cortex [396,397], and prevented the inhibitory action of WIN55212-2. In that sense, it mimicked the effect of the genetic deletion of the $\mathrm{CB}_{1} \mathrm{R}$ and we concluded that $\mathrm{O}-2050$ is a useful in vitro $\mathrm{CB}_{1} \mathrm{R}$ antagonist up to the concentration of $1 \mu \mathrm{M}$.

In special cases, a GPCR can assume an inactive structure which would sequester surrounding $G$ proteins rather than allowing their spontaneous dissociation. The probability of this phenomenon is facilitated by certain ligands which are called inverse agonists. The first prototypic $\mathrm{CB}_{1} \mathrm{R}$ antagonist, 1,5-diarylpyrazole SR141716 [230] is in fact an $\mathrm{CB}_{1} \mathrm{R}$ inverse agonist [351,386,392], which means that it triggers responses on its own via the blockade of constitutive (also known as basal) $\mathrm{CB}_{1} \mathrm{R}$ signaling, and the consequent disruption of active intracellular coupling. As a result, the direction of those responses will be perceived as reversed compared to the responses triggered by a true agonist, hence the name inverse agonist. Strikingly, under GTP $\gamma \mathrm{S}$ treatment, $R$ methanandamide inhibited the forced dissociation of $\mathrm{G}_{\text {ail }}{ }^{-}$ GTP $\gamma \mathrm{S}$ and $\mathrm{G}_{\alpha \mathrm{i} 2}-\mathrm{GTP} \gamma \mathrm{S}$ while desacetyllevonantradol precluded the dissociation of $\mathrm{G}_{\alpha i 3}$-GTP $\gamma \mathrm{S}$ and attenuated that of $\mathrm{G}_{\alpha i 1}-\mathrm{GTP} \gamma \mathrm{S}$ from the $\mathrm{CB}_{1} \mathrm{R}$ [386]. This paradoxical observation means that not only an antagonist but also an agonist can behave as an inverse agonist at the same time, i.e., activating a pathway for which it has high intrinsic efficacy while antagonizing other pathways; thus adding to the complexity of biased agonism. Although WIN55212-2 inhibits cyclic adenosine monophosphate (cAMP) accumulation both in vivo in the striatum and in vitro [398], it exhibits a very peculiar case of biased agonism in the presence of $G_{q / 11}$ proteins, as this aminoalkylindole - unlike classical, non-classical or eicosanoid agonists - stimulated $\mathrm{Ca}^{2+}$ outflow from intracellular stores in cell cultures, via the $\mathrm{CB}_{1} \mathrm{R}$ and $\mathrm{G}_{\mathrm{q} / 11}$ proteins [371]. In another study, CP55940 at very low concentrations stimulated cAMP accumulation in 
$\mathrm{CB}_{1} \mathrm{R}$-expressing $\mathrm{CHO}$ cells via pertussis toxin-insensitive $\mathrm{G}$ proteins; however, CP55940 at much higher concentration counteracted the forskolin-induced stimulation of cAMP accumulation [370]. Additional studies in $\mathrm{CHO}$ cells unveiled that the blockade of $\mathrm{G}_{\mathrm{i} / \mathrm{o}}$ by pertussis toxin triggers the association of $\mathrm{CB}_{1} \mathrm{Rs}$ to stimulatory $\mathrm{G}$ proteins (as witnessed by the facilitation by HU210, CP55940, $\Delta^{9}$-THC, WIN55212-2 and anandamide of the forskolin-stimulated rather than the basal cAMP accumulation) [384,399]. These agonist- and concentration-dependent phenomena are termed as promiscuous $G$ protein coupling [400]. There is a surprising twist to the promiscuous $\mathrm{G}$ protein coupling, too, because the intrinsic efficacy of ligands changes dramatically when the $C_{1} R$ couples to $G_{s}$ [384]. In the present example, WIN55212-2 was the most efficacious either to stimulate basal cAMP accumulation or to inhibit forskolin-stimulated cAMP accumulation, while the efficacy of $\Delta^{9}$-THC to stimulate basal cAMP accumulation dropped 30\%, and that of CP55940 was halved and finally, the efficacy of anandamide fell to $1 / 3$ [384].

\subsubsection{Allosteric Modulation of the $C B_{1} R$}

An additional case of receptor-ligand interaction does not directly involve the principal (orthosteric) binding site of the $\mathrm{CB}_{1} \mathrm{R}$. Allosteric modulators can either negatively (negative allosteric modulator, NAM) or positively (positive allosteric modulator, PAM) affect the conformation of the receptor so that orthosteric cannabimimetics display smaller or greater affinity and efficacy at the receptor [401]. The characterization of the first $\mathrm{CB}_{1} \mathrm{R}$-selective synthetic functional PAM compounds, ORG27569, ORG29647, ORG27759 [402] were followed by PSNCBAM-1, which works as a NAM of the $\mathrm{CB}_{1} \mathrm{R}$ both in vitro and in vivo, thus mimicking the effects of a silent $\mathrm{CB}_{1} \mathrm{R}$ antagonist and does not affect the constitutive coupling of the hCB ${ }_{1} R$ [403]. Both the $\mathrm{hCB}_{1} \mathrm{R}$ and the $\mathrm{hCB}_{2} \mathrm{R}$ have a cholesterolinteraction/recognition amino acid sequence consensus (CRAC) in their last 11 -amino acid sequence of the $7^{\text {th }}$ transmembrane helix $\left(\mathrm{TMH}_{7}\right)$, however, the lysine 402 in the $h C B_{1} R$ is substituted with a glycine in the $h_{C B} R$. While cholesterol acts as a NAM on the $h_{C B} R$, the $h C B_{2} R$ is completely insensitive to cholesterol due to the single amino acid difference in the CRAC sequence [404]. Apart from this, there is at least one more cholesterol binding site in the $\mathrm{CB}_{1} \mathrm{R}$ - denoted as cholesterol consensus motif (CCM), which stretches through $\mathrm{TMH}_{1-4}$, and where ORG27569 competes with cholesterol for allosteric modulation [405]. Interestingly though, cholesterol promotes centrifugal $\mathrm{CB}_{1} \mathrm{R}$ trafficking to the lipid rafts of neural processes which are rich in endocannabinoid signalosomes, while the treatment with ORG27569 works the other way around [405]. These also suggest that disturbances in systemic cholesterol homeostasis may eventually affect $\mathrm{CB}_{1} \mathrm{R}$ signaling. Interestingly, the cholesterol-derivative endogenous hormone, pregnenolone provides negative feedback onto $\mathrm{CB}_{1} \mathrm{R}$ signaling in the brain via negative allosteric modulation [406] (see Section 3.3.9).

In contrast, RTI-371 works as a PAM [407], although it was originally meant to be a dopamine transporter (DAT) inhibitor (see also Section 3.3.4.1). Lipoxin A4 has been identified as an endogenous enhancer of anandamide effects at the $\mathrm{CB}_{1} \mathrm{R}$ [408]

\subsubsection{Cannabis Constituents at the $C B_{1} R$ and the $C B_{2} R$}

Interestingly, certain phytocannabinoids differ in their action at the two receptors. The natural stereoisomer of $\Delta^{9}$ THC is (3R,4R)-(-)-trans-delta-1-tetrahydrocannabinol (in early papers it was referred to as $\Delta^{1}$-THC), which is ten-tohundred times more potent than the unnatural $(3 \mathrm{R}, 4 \mathrm{R})-(+)-$ $\Delta^{1}$-THC [409]. $\Delta^{9}$-THC in most assays acts as a partial agonist at the $\mathrm{CB}_{1}$ and $\mathrm{CB}_{2}$ receptors - i.e., it does not elicit the possible maximal effect $[388,410]$, and in some assays proves to be more potent at the $\mathrm{CB}_{2} \mathrm{R}$ than at the $\mathrm{CB}_{1} \mathrm{R}$ (e.g., $[411,412])$. Importantly, $\Delta^{9}$-THC is more potent at the human than the rat $\mathrm{CB}_{1} \mathrm{R}$ (hence contrasting many other cannabimimetics), as concluded from a meta-analysis of pharmacological studies [411]. This increased potency contributes to the observation that a $\Delta^{9}$-THC dose as low as $50 \mu \mathrm{g} / \mathrm{kg}$ is enough to elicit subjective responses in humans while up to 60-times higher dose is required to obtain psychopharmacological responses in the rat [411]. At high concentrations, $\Delta^{9}$-THC may paradoxically antagonize the $\mathrm{CB}_{1} \mathrm{R}$ [410]. One major in vivo metabolite of $\Delta^{9}$-THC, 11hydroxy-THC is suspected to be a fast-acting major psychoactive molecule, in part responsible for the central effects of cannabis when ingested orally, but has low plasma levels after smoking $[188,189]$. In vitro, however, 11hydroxy-THC proved to be less potent and efficacious than $\Delta^{9}$-THC to inhibit $\mathrm{Ca}^{2+}$ uptake in mouse brain synaptosomes [413].

The non-psychoactive CBN [414] is technically an oxidized form of $\Delta^{9}$-THC, and does not bind to either of the two receptors $[412,415]$, although there are reports on CBNinduced minor responses $\left(10 \%\right.$ of that of $\left.\Delta^{9}-\mathrm{THC}\right)$ at the $\mathrm{CB}_{1} \mathrm{R}[267,410]$.

Another major cannabis constituent, $\Delta^{9}$-THCV [416] acts as a potent neutral $\mathrm{CB}_{1} \mathrm{R}$ antagonist and as a partial $\mathrm{CB}_{2} \mathrm{R}$ agonist both in vitro and in vivo $[415,417]$. Yet, $\Delta^{9}$ THCV at high doses $(\geq 3 \mathrm{mg} / \mathrm{kg})$ produces in vivo $\Delta^{9}$-THClike activity, which is perhaps a consequence of in vivo metabolism into 11-hydroxy-THCV [415,417] - a metabolite awaiting further characterization. Interestingly, both $\Delta^{9}$-THC and $\Delta^{9}$-THCV acted rather like inverse agonists on VGCC $\mathrm{Ca}^{2+}$ currents in $\mathrm{CB}_{2} \mathrm{R}$-expressing cells [376], suggesting that low-efficacy cannabinoid agonists can compete with highefficacy (full) agonists for the binding site, thereby reducing the overall response.

(-)-Cannabidiol (CBD), the non-classical nonpsychoactive ingredient of cannabis binds poorly both to the $\mathrm{CB}_{1} \mathrm{R}$ and the $\mathrm{CB}_{2} \mathrm{R}$, and exhibits no agonist activity or psychoactivity [267]. CBD is not a listed substance, and it is believed to have a plethora of beneficial therapeutic effects [267]. However, the pharmacological profile of CBD is largely unknown at human cannabinoid receptors despite the fact that clinical trials have been carried out with this molecule [411,418]. As an example, low micromolar CBD has been found to induce endothelium-dependent vasorelaxation in the human mesenteric arteries partly via $\mathrm{CB}_{1} \mathrm{R}$ activation [419]. Strikingly, in the presence of other agonists, CBD can also act as a highly potent antagonist at both receptors, and in some assays, CBD exhibited inverse agonism $[415,420]$. CBD content in cannabis also inversely correlates with the psychotogenic properties of $\Delta^{9}$-THC [175]. Intriguingly, CBD was almost two orders of magnitude less potent to displace $\left[{ }^{3} \mathrm{H}\right] \mathrm{CP} 55940$ binding in $\mathrm{CB}_{1} \mathrm{R}$-rich brain membranes and in $\mathrm{hCB}_{2} \mathrm{R}$-transfected $\mathrm{CHO}$ 
cell membranes than to prevent CP55940 from stimulating $\left.{ }^{35} \mathrm{~S}\right]$-GTP $\gamma \mathrm{S}$ binding in the same preparations [420]. This strongly argues against the idea that CBD surmounts agonists at the orthosteric binding pocket; instead, CBD seems to modulate $\mathrm{CB}_{1 / 2} \mathrm{R}$ function through an allosteric binding site. Indeed, a new study claims that CBD is in fact a NAM [421]. The trans-isomer of the bicyclic sesquiterpene, $\beta$-caryophyllene is a $\mathrm{CB}_{2} \mathrm{R}$-selective ligand of major therapeutic potential found in high concentrations in essential hemp oil [422,423].

Finally, CBC acts in vivo as a low-potency $\Delta^{9}$-THCmimetic [267,424,425], but its pharmacological profile is far from understood.

\subsubsection{Subtype-preferring Endocannabinoids and Synthetic Ligands}

Among the endocannabinoids, 2-arachidonoylglyceryl ether (noladin ether) [412,426], $N$-arachidonoyl dopamine (NADA) [427], and oleamide [428] are activators of the $\mathrm{CB}_{1} \mathrm{R}$ but not the $\mathrm{CB}_{2} \mathrm{R}$ in the nanomolar range, although they also have other cognate receptors (see later), thus they are not considered "CB ${ }_{1}$ R-selective". Docosatetraenylethanolamide (DEA) and homo- $\gamma$ linolenylethanolamide (HEA) are additional, illcharacterized anandamide-like endocannabinoids produced in brain cells, being selective for the $\mathrm{CB}_{1} \mathrm{R}$ at least over the $\mathrm{CB}_{2} \mathrm{R}$ [429-431]. O-arachidonoylethanolamine (virodhamine) is a potent full agonist of the $\mathrm{CB}_{2} \mathrm{R}$, but acts as a biased ligand (a low-potency partial agonist as well as antagonist) at the $\mathrm{CB}_{1} \mathrm{R}[412,432]$.

Additionally, numerous highly selective and potent synthetic $\mathrm{CB}_{1} \mathrm{R}$ and $\mathrm{CB}_{2} \mathrm{R}$ ligands are also available. For example, metabolically stable anandamide analogs, $(R)-(+)-$ methanandamide [433] and arachidonoyl-2'chloroethylamide (ACEA) [434] (Fig. 1) were among the first synthetic compounds with considerable selectivity toward the $\mathrm{CB}_{1} \mathrm{R}$ over the $\mathrm{CB}_{2} \mathrm{R}$ in the nanomolar range, but unfortunately, they may exhibit agonist activity at other receptors, too. For unknown reasons, there is a paucity of commercially available, sufficiently selective and efficacious $\mathrm{CB}_{1} \mathrm{R}$-selective agonists. As for the $\mathrm{CB}_{1} \mathrm{R}$-selective antagonists, SR141716A (rimonabant, Acomplia ${ }^{\mathrm{TM}}$ ) and its analogs, AM251 [435] and AM281 [436] are the best known inverse agonist examples, but SR141716A and AM251 were also reported to bind to the GPR18 and the GPR55 (see below). Another SR141716A analog, AM6545 is a nonbrain-penetrant silent $\mathrm{CB}_{1} \mathrm{R}$ antagonist with promising therapeutic profile [262], whereas O-2050 and NESS0327 are brain-penetrant neutral antagonists of the $\mathrm{CB}_{1} \mathrm{R}$ [393,394].

As for the $\mathrm{CB}_{2} \mathrm{R}$-selective ligands, there are a number of structurally different molecules commercially available. JWH133 (Fig. 1) is a potent $\mathrm{CB}_{2} \mathrm{R}$-preferring $\Delta^{8}$-THCderivative [437], although it has considerable affinity in the high nanomolar range for the $\mathrm{CB}_{1} \mathrm{R}$, too. HU308 is slightly less potent than JWH133 but is highly selective for the $\mathrm{CB}_{2} \mathrm{R}$ [438] over the $\mathrm{CB}_{1} \mathrm{R}$, while the SR141716A analog diarylpyrazole GP1a is a hyperpotent $\mathrm{CB}_{2} \mathrm{R}$ agonist [439]. AM630 (iodopravadoline; [440]), SR144528 [441] and JTE907 [442] are the most common antagonists/inverse agonists of the $\mathrm{CB}_{2} \mathrm{R}$. Interestingly, the so-called "inactive enantiomer" of WIN55212-2, i.e., WIN55212-3 is in fact a silent $\mathrm{CB}_{1} \mathrm{R}$ antagonist and a $\mathrm{CB}_{2} \mathrm{R}$ inverse agonist [443].

\subsection{4. $C B_{1} R$ and $C B_{2} R$ Accessory Proteins}

The regulation and termination of $\mathrm{CB}_{1} \mathrm{R}$ signaling is controlled by accessory proteins such as GPCR kinases (GRKs), $\beta$-arrestins, GPCR-associated sorting protein 1 (GASP1) and cannabinoid receptor interacting protein CRIP1a [444]. Agonist-induced GPCR desensitization rather than the constitutive internalization starts with the phosphorylation by GPCR kinases (GRKs), which is followed by $\beta$-arrestin binding and the consequent prevention of further G protein coupling, as well as GRKand $\beta$-arrestin-mediated activation of preferred pathways $[445,446]$. $\beta$-arrestin2 (also termed as arrestin3 without $\beta$ ) has a major role in the agonist-induced desensitization and internalization of the $\mathrm{CB}_{1} \mathrm{R}[376,447,448]$, which contributes to the transient nature of the $\mathrm{CB}_{1} \mathrm{R}$ activation of downstream pathways [449], and probably is also involved in cannabinoid tolerance [450-452]. In $\beta$-arrestin2 knockout mice, the density of $\beta$-arrestin 1 and $\mathrm{CB}_{1} \mathrm{Rs}$ remained unchanged, but a redistribution of $\mathrm{CB}_{1} \mathrm{R}$ pools was observed to favor the synapses over the somatodendritic compartments, and $\Delta^{9}$-THC became less potent in the absence of $\beta$-arrestin2 [453]. Interestingly, cannabimimetics promote desensitization at rates not necessarily proportional to their efficacy: noladin ether and WIN55212-2 desensitize the $\mathrm{CB}_{1} \mathrm{R}$ rapidly while $\Delta^{9}$-THC and anandamide do it more slowly ( 5\%/min) [454]. It is believed that $\beta$-arrestin1 has further roles as it can mediate short term activation of ERK1/2, MEK1/2, dual specificity mitogen-activated protein kinase kinase 1/2 (MAP2K1/2) and proto-oncogene tyrosineprotein C-terminal kinase Src (c-Src) [455].

In contrast to $\beta$-arrestins, chlatrin heavy chain is required for both the constitutive and the agonist-induced internalization of the $\mathrm{CB}_{1} \mathrm{R}$ [448]. An additional protein, Src homology 3-domain growth factor receptor-bound 2-like (endophilin) interacting protein 1 (SGIP1) prevents the endocytosis of the activated $\mathrm{CB}_{1} \mathrm{R}$ and alters its signaling in a biased manner [456]. Internalized $\mathrm{CB}_{1} \mathrm{Rs}$ are not recycled, but instead, undergo lysosomal degradation [457]. Some suggest that an additional intracellular pool of $\mathrm{CB}_{1} \mathrm{Rs}$ apparently does not participate in the repopulation of the plasmalemma [457], and may participate in intracellular endocannabinoid signaling including the regulation of mitochondrial metabolism $[458,459]$. The trafficking of $\mathrm{CB}_{1} \mathrm{Rs}$ into the lysosomes involve GASP1-dependent sorting, thus viral reduction and genetic ablation of GASP1 attenuates the downregulation of the $\mathrm{CB}_{1} \mathrm{R}$ and mitigates behavioral tolerance after chronic or long-term treatment with WIN55212-2 [460,461]. While this process is probably more typical for axons and transfected HeLa or Neuro-2 cells, somatodendritic $\mathrm{CB}_{1} \mathrm{Rs}$ may be regulated differently in other neurons. Indeed, in cultured hippocampal GABAergic neurons, $\mathrm{CB}_{1}$ Rs undergo constant endocytosis and recycling, in a fashion dependent on eps15, dynamin1, dynamin2, rab5 and chlatrin [231]. Furthermore, this process can be halted by a $\mathrm{CB}_{1} \mathrm{R}$ inverse agonist, AM281 (analogous to SR141716A), leading to the abundance of $\mathrm{CB}_{1} \mathrm{Rs}$ in somatodendritic membranes [231]. This mechanism can explain some of the inverse agonism as it increases the number of cell-surface $\mathrm{CB}_{1} \mathrm{Rs}$, but it is not clear if these receptors are readily available to spontaneously couple to 
second messengers or they are kept in inactive state by the inverse agonist. Some new data may suggest that these receptors could be functional after all, because a low dose of AM281 (0.5 mg/kg) administered for 14 days caused conditioned place preference for rats [462]. Finally, the adaptor protein AP-3 $\delta$ has been shown in hippocampal and other neurons to assist in the direct transport of $\mathrm{CB}_{1} \mathrm{Rs}$ into late endosomes/lysosomes, where the $\mathrm{CB}_{1} \mathrm{R}$ - somewhat surprisingly - is still active and couples to heterotrimeric $\mathrm{G}$ proteins [463].

CRIP1a (164 amino acids) and CRIP1b (128 amino acids) are splice variants of the cannabinoid receptor interacting protein gene in primates, while only CRIP1a has been detected in lower vertebrates and is highly expressed in $\mathrm{CB}_{1} \mathrm{R}$-rich areas such as the brain [464]. Both isoforms interact with the intracellular C-terminus of the $\mathrm{CB}_{1} \mathrm{R}$, and apparently the last 9 amino acids of the $\mathrm{CB}_{1} \mathrm{R}$ are essential for this coupling [464]. Only CRIP1a has a palmitoylation site that predicts membrane localization for this isoform. Indeed, CRIP1a rather than CRIP1b prevented the constitutive inhibition of $\mathrm{Ca}^{2+}$ channels in superior cervical ganglion neurons, while neither isoform affected significantly the binding constant $\left(K_{d}\right)$ of the $\mathrm{CB}_{1} \mathrm{R}$ [464]. Accordingly, a recent study claims that CRIP1a overexpression can effectively inhibit both constitutive and agonist-induced $\mathrm{CB}_{1} \mathrm{R}$ activity as well as subchronic agonist treatment-induced downregulation but not desensitization of the $\mathrm{CB}_{1} \mathrm{R}$ in HEK293, N18TG2 and hippocampal neuronal cultures [465]. These findings were discordant with another study which showed that CRIP1a overexpression enhances $\mathrm{CB}_{1} \mathrm{R}$-mediated $\mathrm{G}$ protein activation and the consequent inhibition of excitatory currents in the hippocampus [466]. The probable explanation to this discrepancy lies in a third study which demonstrated that CRIP1a stimulates $\mathrm{CB}_{1} \mathrm{R}$ coupling to some of the $G$ proteins but inhibits it to others: In N18TG2 cells, the expression levels of CRIP1a were inversely proportional to the extent of $C_{1} R$-mediated $G_{i 3}$ and $G_{0}$ activation and ERK phosphorylation, and positively proportional to $G_{i 1 / 2}$ activation [467]. While in this study the authors did not find CRIP1a overexpression to affect $\mathrm{CB}_{1} \mathrm{R}$ signaling, they observed that the cannabimimetic CP55940 showed enhanced efficacy and potency in CRIP1a-deficient cells [467]. These studies altogether highlight gaps in our understanding of the exact roles of CRIP1a in $\mathrm{CB}_{1} \mathrm{R}$ signaling, which can be a consequence of the overexpression protocol which can trigger artificial (contrariwise) responses. This hypothesis is bolstered by a study where the authors first reproduced previous findings that the cannabimimetic WIN55212-2 rather than the $\mathrm{CB}_{1} \mathrm{R}$ inverse agonist/antagonist SR141716A mitigated excitotoxic cell death in cultured cortical neurons [468]. CRIP1a overexpression above the normal (basal) CRIP1a density did not affect glutamate toxicity per se, but strikingly, it reverted the role of the agonist and the antagonist, and thus SR141716A conferred protection against excitotoxicity, rather than WIN55212-2 [468]. The possible role of CRIP1a in excitotoxicity has been further supported by the finding that the expression of CRIP1a and the $C_{1} R$ was lower in sclerotic hippocampal tissue obtained from epileptic patients [469], but whether these were active or passive events in the pathogenesis of neurodegeneration is not fully understood.

The $\mathrm{CB}_{2} \mathrm{R}$ has been also found to interact with $\beta$ arrestin $[470,471]$. Notably, the link between $\beta$-arrestin2 and internalization is not so linear than in the case of the $C_{1} R$. For instance, it was found in one of the above studies [376] that both WIN55212-2 and CP55940 induced $\beta$-arrestin2 recruitment to the membranes of $\mathrm{CB}_{2} \mathrm{R}$-transfected $\mathrm{HEK} 293$ cells, but only CP55940 rather than WIN55212-2 or $\Delta^{9}$-THC triggered $\mathrm{CB}_{2} \mathrm{R}$ internalization (resulting in a perinuclear accumulation of the $\mathrm{CB}_{2} \mathrm{R}$, contrasting the cytoplasmic $\mathrm{CB}_{1} \mathrm{R}$ redistribution), and even more strikingly, WIN552122 and $\Delta^{9}$-THC prevented CP55940 from $\mathrm{CB}_{2} \mathrm{R}$ internalization, acting as antagonists. This is also consistent with $\Delta^{9}$-THC being a rather weak $\mathrm{CB}_{2} \mathrm{R}$ agonist that can likely act as an antagonist in the presence of another more efficacious agonist [280].

\subsubsection{GPR18}

The recently "deorphanized" G protein-coupled receptor 18 (GPR18 and not GPCR18) was first reported in 1997 as a protein highly expressed in spleen and testis [472], and is currently emerging as a possible candidate of the endocannabinoid receptor family [473]. This receptor has been identified with the elusive abnormal-cannabidiol (abnCBD) receptor of the past $[474,475]$, although it is feasible that some of the reported observations with the atypical cannabinoid, abn-CBD were in fact mediated by the GPR55 (see Section 3.2.6). Endogenous agonists for GPR18 are $\mathrm{N}$ arachidonoylglycine (NAGly) [473,476-479] and the nonclassical eicosanoid, resolvin $2 \mathrm{D}$, which is a COX-2 product involved in the resolution of acute inflammation [480]. Notably, NAGly has been proposed to be the endogenous agonist for additional GPCRs, namely the GPR82 and the GPR141, respectively [481].

$\Delta^{9}$-THC, as well as the atypical cannabinoids, abn-CBD and O-1602 are also potent full agonists at the GPR18, together with the endocannabinoid, anandamide and its metabolically resistant analog, ACPA [473,474,478,482485]. Notably, another study questioned whether the above ligands truly serve as agonists for GPR18 [486]. WIN552122, CP55940, JWH133 and JWH015 do not activate the GPR18 [478]. AM251, SR141716A and CBD are non-selective antagonists (as having affinity to other cannabinoid receptors, see above), while the atypical cannabinoid, O-1918 is apparently a selective GPR18 antagonist [474,475,478,485,487].

GPR18 signalizes through pertussis toxin-sensitive $G$ proteins, $\mathrm{PI}_{3} \mathrm{~K}$, p42/44 mitogen-activated protein kinase and Akt [487]. Among the tested ligands, only $\Delta^{9}$-THC was capable of inducing $\beta$-arrestin recruitment in $\mathrm{CHO}$ cells transfected with GPR18.

\subsubsection{GPR55}

The "deorphanized" G protein-coupled receptor 55 (GPR55) is the fourth relevant metabotropic receptor that interacts with cannabis constituents as well as synthetic and endogenous cannabinoids. Its discovery was reported in 1999 [488], and it was initially classified as an orphan GPCR, because its endogenous ligands - lysophospholipids - were discovered only a few years later. This 7 transmembrane-domain receptor has 319 amino acids in humans and 327 in mice, and its gene is probably exclusive to mammals [489]. GPR55 exhibits low sequence homology to $\mathrm{CB}_{1} \mathrm{Rs}$ and $\mathrm{CB}_{2} \mathrm{Rs}(13-15 \%)$ [489,490]. GPR55 can couple to $G_{\alpha 13}[412,491], G_{\alpha 12}$, or $G_{\alpha q}[492]$, resulting in the elevation of $\left[\mathrm{Ca}^{2+}\right]_{i}$ or in the activation of ERK, p38 MAPK, 
PLC, Ras homolog gene family member A (RhoA), and Rho-associated protein kinase (ROCK) [490-495].

L- $\alpha$-lysophosphatidylinositol (LPI), 2-arachidonoyl-snglycero-3-phosphoinositol (2-AGPI) and lysophosphatidyl$\beta$-D-glucoside (LysoPtdGlc) have been identified as the most potent and efficacious endogenous activators of the GPR55 [496-498]. True endocannabinoids including 2-AG, anandamide, noladin ether as well as additional "endocannabinoid-like" molecules, virodhamine, palmitoylethanolamide and oleoylethanolamide are also potent activators of the GPR55, although differences are reported throughout different experimental approaches $[412,489,490,499-502]$. In fact, the pharmacology of the GPR55 is the most complex and the least understood among the cannabinoid receptors, because the same ligands can behave as an agonist, antagonist or a neutral ligand in different assays or even in the same assay, depending on the presence or absence of an endogenous agonist $[489,503,504]$. It is believed that the GPR55 possesses an allosteric modulator site where certain exogenous ligands either have no effect on their own or seemingly activate the GPR55 assuming that it is unoccupied by endogenous ligands, but in the presence of endogenous GPR55 activator lipids, these exogenous ligands become antagonists [412,492-494,503-507]. Collating data from the above studies, we can draw a general pharmacological profile of the GPR55, keeping in mind that not all agonists would behave consistently in all the assays. (1) Phytocannabinoids $\Delta^{9}$-THC, $\Delta^{9}$-THCV and CBDA per se act as weak (partial) agonists at the GPR55, but they inhibit LPI-induced GPR55 activation, together with CBD and CBDV [508] and CBGV. (2) Synthetic analogs and derivatives of phytocannabinoids, that is, the classical cannabimimetic HU210, the nonclassical cannabimimetic CP55940, and the atypical cannabinoids, abn-CBD and O-1602 exhibit (partial) agonism of varying potencies, while the $\mathrm{CB}_{2} \mathrm{R}$ agonist analogs, HU438 and HU914 antagonize LPI-induced ERK1/2 phosphorylation at the GPR55. (3) Among the $\mathrm{CB}_{2} \mathrm{R}$-preferring agonists, the naphthoylindole-type JWH015 can activate the GPR55 while the classical-type JWH133 behaves as an inverse agonist (i.e., it inhibits both basal and LPI-induced GPR55 activity). (4) The hydrolysis-resistant anandamide analog $R$-methanandamide also activates the GPR55. (5) Finally, the $\mathrm{CB}_{1} \mathrm{R}$-preferring inverse agonists, the 1,5-diarylpyrazole compounds AM251 and SR141716A in most cases act as low-potency agonists or allosteric activators, but again, they antagonize LPI. The term "lowpotency" stands for $\mathrm{EC}_{50}$ and $\mathrm{IC}_{50}$ values in the micromolar range. Cannabinoids with such low potency are of minor pharmacological importance, because in the low micromolar range, the number of their novel binding sites and off-target effects increase drastically [381,509], while their solubility in assay media and body fluids becomes an issue. Nevertheless, the considerably overlapping pharmacological profile of the four above cannabinoid receptors, i.e., $C_{1} R$, $\mathrm{CB}_{2} \mathrm{R}$, GPR18 and GPR55 prompts caution when interpreting observations. The use of GPR55-selective antagonists such as CID16020046 [510] and ML193 [511] and the GPR18-selective antagonist, O-1918 (see Section 3.2.5) certainly can facilitate the identification of the two novel deorphanized receptors in bioassays.

Altogether, the inconvenient question whether the GPR18 and the GPR55 are bona fide members of the endocannabinoid system challenges the classical view of receptor pharmacology, and soon a reclassification of signaling systems based on domain-specific signalosomes will be inevitable.

\subsubsection{Transient Receptor Potential Channels}

The transient (light-induced) receptor potential (TRP) channel superfamily accommodates 27 polymodal sensor cation channels divided among 6 groups in humans [512]. Among these, 4 families have members with which cannabinoids and cannabis constituents interact [380,509,512]. As they are involved in nociception, analgesia and immune modulation, cannabis constituents are well-positioned to modulate pain sensation and inflammation through these channels.

\subsubsection{TRP Vanilloid Family}

The TRP vanilloid family (TRPV) has 6 members, among which the $T R P V_{1} R$, the $T R P V_{2} R$ and the $T R P V_{4} R$ are closely related channels [512]. The $\mathrm{TRPV}_{1} \mathrm{R}$ is a principal noxious stimulus receptor of the body, as it is activated by heat, protons, phytotoxins such as capsaicin, piperine, curcumin and gingerol, and animal venoms [512514]. $\mathrm{TRPV}_{1} \mathrm{R}$ is also activated by many lipids [515] including endocannabinoids, anandamide [516], NADA [517] and 2-AG [518]. Albeit the lack of 2-AG agonism or the intracellular ligand binding site were widely accepted dogmas for quite a while, now it is clear that the binding site of the $T_{R P V} R$ faces the extracellular side [519,520]. Furthermore, it appears that the $\mathrm{TRPV}_{1} \mathrm{R}$ is sufficient to serve as an endocannabinoid membrane transporter, that is, the pore of the $T R P V_{1} R$ permits the passage of anandamide through the cell membrane [521].

Among the cannabis constituents (in rank order of efficacy) CBGA, CBGV, $\Delta^{9}$-THCV, CBD, and CBG provoked sound $\mathrm{Ca}^{2+}$ entry in HEK293 cells transfected with the human $\mathrm{TRPV}_{1} \mathrm{R}$ with $\mathrm{EC}_{50}$ values below $5 \mu \mathrm{M}$, while $\Delta^{9}$ THC, $\Delta^{9}$-THCA, CBN and CBC failed to elicit $\left[\mathrm{Ca}^{2+}\right]_{\mathrm{i}}$ elevation at reasonable concentrations [190,191,522]. An obvious enigma is the apparent lack of CBD pungency, and the answer probably lies in differential responses by the $\mathrm{TRPV}_{1} \mathrm{R}$. In fact, capsaicin evokes small inward currents at the human $\mathrm{TPRV}_{1} \mathrm{R}$ but with high potency in comparison with piperine from the black pepper, which produces large currents but at low potency [523]. In the rat eye-wipe assay, NADA and capsaicin provoked irritation while anandamide and ACEA failed to do so, even though they also activate $\mathrm{TRPV}_{1} \mathrm{R}$ current. It is probable that the cation current evoked by the non-pungent agonists is short-lived due to rapid desensitization; hence it probably does not trigger depolarization that could excite the sensory afferents. Indeed, CBD has been shown to desensitize the $T_{R P V} R$ to the action of capsaicin [190]. Others even found a lack of agonist activity for CBD at the rat $\mathrm{TRPV}_{1} \mathrm{R}$ [524]. Furthermore, CBD has been claimed to be an inhibitor of the elusive anandamide or endocannabinoid membrane transporter (EMT) [190], and EMT activity can be attributed at least in part to $\mathrm{TRPV}_{1} \mathrm{R}$-dependent mechanisms [521]. Synthetic cannabinoid ligands, such as HU210 and JWH015 were also reported to act as low potency partial agonists at the $\mathrm{TRPV}_{1} \mathrm{R}$. Altogether, it is easy to agree on the inclusion of the $\mathrm{TRPV}_{1} \mathrm{R}$ in the cannabinoid receptor family. 
The $\mathrm{TRPV}_{1} \mathrm{R}$ has been consistently shown to participate in synaptic plasticity (notably, long-term depression or LTD) in the brain of adolescent and young adult laboratory rodents [299,525]. Surprisingly, SR141716A but not AM251 was shown to block TRPV ${ }_{1} R$ in the low micromolar range [525], indicating that some forms of LTD, previously thought to be $\mathrm{CB}_{1} \mathrm{R}$-dependent, might have been in fact $\mathrm{TRPV}_{1} \mathrm{R}$-dependent [525]. Additionally, SR141716A was shown to enhance neurogenesis via the $\mathrm{TRPV}_{1} \mathrm{R}$ [526] (see also Section 3.3.7). Though the inactivation of the $\mathrm{TRPV}_{1} \mathrm{R}$ has anxiolytic therapeutic potential [527], the presynaptic neuromodulator role of the $\mathrm{TRPV}_{1} \mathrm{R}$ remains elusive in the adult brain of laboratory rodents $[512,528]$. Some studies prompt the hypothesis that $\mathrm{TRPV}_{1} \mathrm{Rs}$ are post-synaptic and probably intracellular. For instance, indirect activation of these putative post-synaptic intracellular $\mathrm{TRPV}_{1} \mathrm{Rs}$ by increasing intracellular anandamide levels impairs DAGL $\alpha$-mediated mobilization of 2-AG both in the striatum [529] (Fig. 8) and in the hippocampus [530].

The $\mathrm{TRPV}_{2} \mathrm{R}$ is activated by temperatures above $53^{\circ} \mathrm{C}$, and is also involved in pathological conditions and pain sensation [512]. One publication reported that several cannabinoids activate the $\mathrm{TRPV}_{2} \mathrm{R}$ with high efficacy albeit low micromolar potency [524]. In order of efficacy, $T_{R P V} R$ is activated by CBD, $\Delta^{9}$-THC, CBN, nabilone, 11-hydroxyTHC, CP55940, and to a lesser extent, by 2-AG, while anandamide had no effect [524]. A later work reported that additional cannabis constituents activated the rat $\mathrm{TRPV}_{2} \mathrm{R}$ with the following rank order of efficacy: $\mathrm{CBGV} \approx \Delta^{9}$ $\mathrm{THCV} \approx \mathrm{CBG}>\Delta^{9}-\mathrm{THCA}>\Delta^{9}-\mathrm{THC} \approx \mathrm{CBDV} \geq \mathrm{CBD} \approx$ $\mathrm{CBN}$. The potency of these compounds ranged between 650 $\mathrm{nM}\left(\Delta^{9}-\mathrm{THC}\right)$ and $19 \mu \mathrm{M}(\mathrm{CBN})[191]$.

\subsubsection{Other TRPs from the Ankyrin, Canonical and Melastatine Families}

The $\mathrm{TRPA}_{1} \mathrm{R}$ is a sole member of its TRP ankyrin family [512]. TRPA $A_{1} R$ is a nonspecific chemosensor activated by a wide variety of irritants including allyl isothiocyanate, the pungent substance of mustard, horse radish and wasabi as well as cold $\left(<17^{\circ} \mathrm{C}\right)$ [512,531,532]. The first studies detected that cannabinoids including WIN55212-2, CP55940, CBD and $\Delta^{9}$-THC activate the $\mathrm{TRPA}_{1} \mathrm{R}$ with efficacies comparable or exceeding that of allyl isothiocyanate [524,531]. Later studies established the following rank order of efficacies: $\Delta^{9}$-THCV (243\% of the effect of $100 \mu \mathrm{M}$ allyl isothiocyanate) $>$ THCV acid $(\mathrm{THCVA})>\mathrm{CBGA}>\mathrm{CBGV}>\mathrm{CBD}>\Delta^{9}-\mathrm{THC} \approx \mathrm{CBC} \approx$ CBDA $>$ CBN $[191,533]$. The most potent among them was CBC $\left(\mathrm{EC}_{50}<100 \mathrm{nM}\right), \mathrm{CBD}(110 \mathrm{nM}), \Delta^{9}$-THC (230 nM) and most of the remaining cannabinoids also had potencies in the high nanomolar-low micromolar range [191,533].

The canonical TRP channel family (TRPC) can be regarded as the ionotropic counterpart of the above detailed phospholipid receptor GPRs, because they are activated by various lipids including DAG, phosphatidylinositols, or lysophospholipids, as well as light mechanical stimuli [512]. The gene of the $\mathrm{TRPC}_{1} \mathrm{R}$ was the first mammalian TRP gene to be discovered two decades ago, but the role of this $\mathrm{Ca}^{2+}$ channel still remains elusive [512]. It is widely expressed throughout the mammalian body, and probably forms heteromeric complexes with other TRP members [512]. Therefore, the physiopharmacology of the $\mathrm{TRPC}_{1} \mathrm{R}$ remains ill-defined, which is further complicated by the lack of overt phenotype in the $\mathrm{TRPC}_{1} \mathrm{R}$-/- mice. Of course, cannabis has to poke its nose into the $\mathrm{TRPC}_{1} \mathrm{R}$ channel, too: it was reported that low micromolar $\Delta^{9}$-THC, CBN, HU210 but not CBD or CP55940 induce sustained $\mathrm{Ca}^{2+}$ rise in immune cells [534,535]. The concentration-response curve of $\Delta^{9}$-THC was inverse U-shaped. Unfortunately, it is not clear whether the underlying $\mathrm{TRPC}_{1} \mathrm{R}$ phenotype was a homodimer (as some believe that this would not be functional [512]) or a heterodimer with other TRP channels, and thus, which of the existing isoforms were responsible for the underlying effects. Interestingly, low micromolar SR141716A and SR144528 both acted as antagonists at the $\mathrm{TRPC}_{1} \mathrm{R}$ even in splenocytes from $\mathrm{CB}_{1} \mathrm{R}$-/- and $\mathrm{CB}_{2} \mathrm{R}$-/- mice, indicating that these antagonists possess numerous additional targets besides the $\mathrm{CB}_{1} \mathrm{R}$ or the $\mathrm{CB}_{2} \mathrm{R}$.

The melastatin TRP family (TRPM) inherited its denomination by the melanoma suppressor TRP gene. $\mathrm{TRPM}_{8}$ is also a cold-sensitive channel activated by low temperature $\left(<22^{\circ} \mathrm{C}\right)$ and by "refreshing" agents such as eucalyptol, menthol and icilin [512,532]. Interestingly, $\mathrm{TRPV}_{1} \mathrm{R}$ activators act as antagonists at the $\mathrm{TRPM}_{8}$ channel: capsaicin, resiniferatoxin, NADA and anandamide all inhibit icilin- and menthol-evoked responses [533,536]. A subsequent study characterized the $\mathrm{IC}_{50}$ values for cannabis constituents ( $\left.\mathrm{IC}_{50}, 60 \mathrm{nM}-4.8 \mu \mathrm{M}\right)$, and in fact major and minor cannabinoids all abolished responses to icilin with the following rank of potencies: $\mathrm{CBD}>\mathrm{CBG}>\Delta^{9}-\mathrm{THCA}>\Delta^{9}-$ $\mathrm{THC}>\mathrm{CBN}>\Delta^{9}-\mathrm{THCV}>\mathrm{CBDV}>\mathrm{CBGA}>\mathrm{THCVA}>$ CBGV > CBDA [191].

\subsubsection{Additional Cannabinoid Receptor Candidates}

The following receptors are considered to have minor or no significance in mediating the effects of cannabis constituents. There are two additional metabotropic receptors related with the endocannabinoid system, GPR35 [537,538] and GPR119 [380,539,540], which are activated by numerous endogenous lipids including anandamide, but to our knowledge, there is no evidence for their direct interaction with Cannabis constituents. The endogenous agonists of GPR35 (a receptor expressed in the brain) are kynurenic acid (KynA), together with 2oleoyllysophosphatidic acid and chemokine CXCL17 [490]. GPR119 is activated by oleoyllysophosphatidylcholines and the GPR55 agonist, oleoylethanolamide [538].

\subsection{The Biomedical Significance of Cannabinoids}

This part of our review briefly outlines the distribution and the functions of cannabinoid receptors. In conjunction with this, we evaluate the therapeutic potential of cannabis constituents as well as the health consequences of cannabis abuse. Below, we lay emphasis on the (patho)physiology of the brain as this organ is the fundamental target of cannabis.

\subsubsection{The Cellular and Tissue Distribution of Cannabinoid Receptors}

The $\mathrm{CB}_{1} \mathrm{R}$ has high expression (mRNA levels) and high density (protein levels) in the brain, while at lower levels, it is also expressed in adrenal gland, heart, lung, prostate, uterus, ovary, testes, bone marrow, thymus and tonsils [338]. Although there is a paucity of large-scale studies with modern techniques on $\mathrm{CB}_{1} \mathrm{R}$ distribution in non-neuronal tissues, we can conclude from the literature that $\mathrm{CB}_{1} \mathrm{Rs}$ are 
much more widely distributed - albeit at low density among cells and tissues than thought before, and often coexist with other cannabinoid receptors (see Section 3.3.5.1). Even though it is tempting to speculate that the amplitude of $\mathrm{CB}_{1} \mathrm{R}$-mediated effects are in close correlation with the local density of the $\mathrm{CB}_{1} \mathrm{R}$ protein, and therefore $\mathrm{CB}_{1}$ Rs have little to do with peripheral tissues, this is far from the truth. The outcome of $\mathrm{CB}_{1} \mathrm{R}$ activation is more likely dependent on the efficacy of coupling with the signal amplification by the second messenger systems. An elegant example for this has been presented in hippocampal GABAergic neurons where $\mathrm{CB}_{1}$ Rs inhibited axon potential-evoked $\mathrm{Ca}^{2+}$ transients in a fashion independent from their density at individual axon boutons [541].

In the brain, $\mathrm{CB}_{1} \mathrm{R}$ expression and protein densities are highest in the cerebellar molecular layer, substantia nigra pars reticulata (containing the $\mathrm{CB}_{1} \mathrm{R}$-laden terminals of the striatonigral pathway), globus pallidus externa and interna, inner granule cell layer of the olfactory bulb, anterior olfactory nucleus, layers II-III, Va and VI of the cerebral cortex (in humans, the highest levels were found in the cingulate gyrus, frontal, secondary somatosensory and motor cortices), hippocampus, as well as in the dorsolateral striatum, while moderate levels of $\mathrm{CB}_{1} \mathrm{R}$ expression are found in the hypothalamus and ventral striatum/nucleus accumbens, and finally, low $\mathrm{CB}_{1} \mathrm{R}$ levels can be found in the brainstem with a lack of $\mathrm{CB}_{1} \mathrm{Rs}$ in the respiratory control centers [286,335,396,410,542-550]. These explain why cannabis has mild effects on cardiovascular and respiratory functions [551]. Fig. 9 illustrates the distribution of $\mathrm{CB}_{1} \mathrm{R}$ immunoreactivity in a mediansagittal slice of the adult rat brain and in coronal slices of the adult mouse brain.

The $\mathrm{CB}_{2} \mathrm{R}$ was originally identified as a protein with no expression in the brain and high density in the immune cells and tissues [276]. As discussed later, an emerging body of evidence supports now physiological and pathological roles for neuronal $\mathrm{CB}_{2} \mathrm{Rs}$ in the brain. For instance, a recent paper clearly argues for that cerebral $\mathrm{CB}_{2} \mathrm{R}$ proteins are mostly neuronal in the healthy brain, while inducible $\mathrm{CB}_{2} \mathrm{Rs}$ become predominant in glia or microglia under disease conditions [555]. In line with this evidence, an ultrasensitive and specific in situ hybridization method called the RNAscope revealed the $\mathrm{CB}_{2} \mathrm{R}$ mRNA predominantly in excitatory and inhibitory neurons throughout the hippocampus with rare expression in microglia [556]. Although the vast majority of presynaptic cannabinoid receptors in the brain can be identified with the $\mathrm{CB}_{1} \mathrm{R}$, there is also pharmacological evidence for presynaptic inhibitory $\mathrm{CB}_{2} \mathrm{Rs}$ in GABAergic terminals of the hippocampus [557] and in its relative vicinity, in the medial entorhinal area [558]. There are also intracellular $\mathrm{CB}_{2} \mathrm{Rs}$ in layer II/III pyramidal cells of the medial prefrontal cortex where their activation results in $\mathrm{IP}_{3} \mathrm{R}$-dependent opening of $\mathrm{Ca}^{2+}$-activated $\mathrm{Cl}^{-}$channels, and a consequent inhibition of neuronal firing [559,560]. $\mathrm{CB}_{2} \mathrm{Rs}$ are also expressed in hippocampal principal neurons, where they modulate the sodium/bicarbonate co-transporter, thereby causing a hyperpolarization of the neuron. This robustly alters the input/output function of CA3 pyramidal cells, and modulates $\gamma$ oscillations in vivo [561]. Apparently, functional $\mathrm{CB}_{2} \mathrm{Rs}$ are also present in hippocampal and cortical astrocytes, and $\mathrm{CB}_{2} \mathrm{R}$ activation both in these neurons and astrocytes increases glucose uptake, which may be a self-regulatory process to provide energy supply to the circuitry under load [562]. Other instances of neuronal $\mathrm{CB}_{2} \mathrm{Rs}$ will be discussed later.

GPR18 is present in cells and tissues related with the immune system including the spleen, thymus, small intestine, leukocytes, lymph nodes, as well as in testis and in gametes. The brain, heart, lung, liver, kidney, pancreas, colon, skeletal muscle and ovary are apparently devoid of significant GPR18 expression [483,563].

The GPR55 is expressed in the following tissues of laboratory rodents and humans: white adipose tissue, certain regions of the central nervous system including the frontal cortex, striatum and spinal cord, adrenal tissue; small intestine, osteoblasts and osteoclasts, vasculature and immune cells $[488,490,498,504,564-569]$. The GPR55 is now in the spotlight of research because it is involved in some specific health conditions including cancer cell proliferation, osteoporosis, obesity, inflammation and neuropathic pain, and neuromodulation - coinciding with its general expression patterns [490,498,504,538,567-570]. Functional presynaptic GPR55 has been also shown in the CA3-CA1 synapses where it transiently potentiates evoked glutamate release from Schäffer collaterals.

For curiosity, we investigated whether $\left[{ }^{3} \mathrm{H}\right] \mathrm{SR} 141716 \mathrm{~A}$ (tritiated rimonabant) binding was subject to displacement by GPR18 and GPR55 ligands in the rat, wild-type (WT) and $\mathrm{CB}_{1} \mathrm{R}$-/- mice. AM251 $(1 \mu \mathrm{M})$ readily displaced $40 \%$ of total $\left[{ }^{3} \mathrm{H}\right]$ SR141716A $(1 \mathrm{nM})$ binding from total brain membranes of rats and WT mice, which was similar in amplitude to the consequence of the genetic deletion of the $\mathrm{CB}_{1} \mathrm{R}$ (Fig. 10C3). The silent $\mathrm{CB}_{1} \mathrm{R}$ antagonist, O-2050 (1 $\mu \mathrm{M})$, the synthetic cannabimimetic, O-2545 $(1 \mu \mathrm{M})$ and the $\mathrm{CB}_{1} \mathrm{R} / \mathrm{TRPV}_{1} \mathrm{R}$ hybrid agonist, NADA $(1 \mu \mathrm{M})$ did not fully displace $\left[{ }^{3} \mathrm{H}\right] \mathrm{SR} 141716 \mathrm{~A}$ from the rat membranes (Fig. 10C3). Surpisingly, the $C B_{1} R$ inverse agonist, LY320135 did not displace $\left[{ }^{3} \mathrm{H}\right] \mathrm{SR} 141716 \mathrm{~A}$ binding at all, suggesting that it is rather a NAM than an orthostheric blocker on the rat $\mathrm{CB}_{1} \mathrm{R}$, since LY320135 already acted as $\mathrm{CB}_{1} \mathrm{R}$ antagonist in the rat brain in two of our studies [396,571]. Similarly to AM251, O-2050 fully displaced $\left[{ }^{3} \mathrm{H}\right] \mathrm{SR} 141716 \mathrm{~A}$ binding from the $\mathrm{CB}_{1} \mathrm{Rs}$ in the mouse brain membranes, and to a smaller extent, O-2545 and WIN55212-2 together with LY320135 all displaced $\mathrm{CB}_{1} \mathrm{R}$ binding in the WT mice. None of the above ligands affected significantly the non$\mathrm{CB}_{1} \mathrm{R}$ dependent residual $\left[{ }^{3} \mathrm{H}\right] \mathrm{SR} 141716 \mathrm{~A}$ binding, because they did not affect $\left[{ }^{3} \mathrm{H}\right] \mathrm{SR} 141716 \mathrm{~A}$ binding in the $\mathrm{CB}_{1} \mathrm{R}-/-$ mice [572] (Fig. 10C3). $N$-oleoyldopamine (OLDA) is another endogenous $\mathrm{TRPV}_{1} \mathrm{R}$ agonist, analogous to NADA but it does not bind to the $\mathrm{CB}_{1} \mathrm{R}$ [512]. Since we mentioned above that SR141716A binds to the TRPV ${ }_{1} R[525,526]$, we now tested if OLDA displaced $\left[{ }^{3} \mathrm{H}\right] \mathrm{SR} 141716 \mathrm{~A}$ binding in rat brain membranes, but unlike NADA, OLDA did not affect $\left[{ }^{3} \mathrm{H}\right] \mathrm{SR} 141716 \mathrm{~A}$ binding. Finally, the GPR18/GPR55 agonist, O-1602 $(10 \mu \mathrm{M})$ also did not displace $\left[{ }^{3} \mathrm{H}\right] \mathrm{SR} 141716 \mathrm{~A}$ at all in none of these animals, suggesting that either $\left[{ }^{3} \mathrm{H}\right] \mathrm{SR} 141716 \mathrm{~A}$ has much lower affinity to $\mathrm{TRPV}_{1} \mathrm{R}, \mathrm{GPR} 18$ and GPR55 than to the $\mathrm{CB}_{1} \mathrm{R}$ and hence at $1 \mathrm{nM},\left[{ }^{3} \mathrm{H}\right] \mathrm{SR} 141716 \mathrm{~A}$ only binds to the $\mathrm{CB}_{1} \mathrm{R}$, or that the three additional targets have negligible density compared to the $\mathrm{CB}_{1} \mathrm{R}$ or both (Fig. 10C3). To our knowledge, this is the first such large-scale analysis of $\left[{ }^{3} \mathrm{H}\right] \mathrm{SR} 141716 \mathrm{~A}$ binding in the rodent brain.

\subsubsection{The Role of $C B_{1}$ Rs in Neurophysiology}


$\Delta^{9}$-THC exerts its rich physiologic, psychotropic and psychotogenic actions via the modulation of fast synaptic transmission in the brain, acting at $\mathrm{CB}_{1}$ Rs which can be present both pre- and post-synaptically in the synapse. Fast synaptic transmission encompasses signaling with glutamate and GABA which activate their fast ionotropic receptors. Later we will discuss several other neuron types that also express $\mathrm{CB}_{1} \mathrm{Rs}$, and thus contribute to the psychoactivity of marijuana. As a short note, here we mention that astrocytes are also equipped with $\mathrm{CB}_{1} \mathrm{Rs}$. $\mathrm{CB}_{1} \mathrm{Rs}$ in astrocytes mediate a plethora of surprising functions, including the regulation of glucose metabolism [458], the impairment of working memory [574], heterosynaptic modulation of synaptic transmission in the hippocampus [575], or the modulation of leptin signaling in the brain [576].

\subsubsection{Presynaptic Plasticity}

Without entering into much detail, at least the half of GABAergic and glutamatergic cells in the brain is endowed with $\mathrm{CB}_{1} \mathrm{Rs}$, which are predominantly localized to axonal terminals, hence those receptors are presynaptic [286,390,547,549,552-554,577-580] (Figs. 6 and 8). Presynaptic $\mathrm{CB}_{1}$ Rs have a major role in various forms of synaptic plasticity in the brain, as reviewed extensively elsewhere [286,299,581,582]. We here briefly overview a few of these mechanisms. One major function of presynaptic $\mathrm{CB}_{1} \mathrm{Rs}$ in glutamatergic nerve terminals is to relay the "message received" confirmation after a successful synaptic event. Namely, glutamate depolarizes the post-synaptic dendrites to cause $\mathrm{Ca}^{2+}$-entry and may also activate metabotropic glutamate receptors $\left(\mathrm{mGluR}_{1 / 5}\right)$, both of which alone or convergently can stimulate DAGL $\alpha$ and the consequent release of 2-AG (Fig. 6). 2-AG then traverses back in the synaptic cleft, and activates presynaptic $\mathrm{CB}_{1} \mathrm{Rs}$, still well within a second, counting from the beginning of glutamate release [317,552,583,584]. This short-term $\mathrm{CB}_{1} \mathrm{R}$ activation will induce the translocation of the $\beta \gamma$ subunit of the respective $\mathrm{G}$ protein in the membrane to inhibit voltagegated $\mathrm{Ca}^{2+}$ channels (VGCCs) and to potentiate GIRKs; both leading to the hypopolarization of the nerve terminal and the transient impediment of further glutamate release [585,586] (Fig. 6). It is possible that the $\mathrm{CB}_{1} \mathrm{R}$ has to form heteromeric complexes with GIRKs [368] and N-type VGCCs [541] to effectively modulate their activity. This mechanism guarantees an optimal strength of synaptic communication: it allows the stimulation of the post-synaptic neuron sufficiently to trigger depolarization, but prevents overstimulation by excitotoxic amounts of glutamate. The activation of $\mathrm{CB}_{1} \mathrm{Rs}$ in GABAergic nerve terminals requires other mechanisms including heterosynaptic activity, metabotropic glutamate or muscarinic receptor activation, or probably lateral endocannabinoid diffusion, because GABA does not produce post-synaptic responses that could mobilize endocannabinoids [286,552,587-589] (Fig. 8).

These trans-synaptic feed-back mechanisms are termed as "retrograde endocannabinoid signaling" [590-592]. Depolarization can trigger trans-synaptic endocannabinoid signaling via post-synaptic $\mathrm{Ca}^{2+}$-entry, which can affect both GABAergic and glutamatergic nerve terminals, hence these phenomena are called depolarization-induced suppression of inhibition or excitation (DSI/DSE) (Figs. 6 and 8). If transsynaptic endocannabinoid mobilization is activated by metabotropic receptor activation, the process is denoted as metabotropic stimulation-induced suppression of inhibition/excitation (MSI/MSE) (Figs. 6 and 8). A special case is the combination of the two: $\mathrm{Ca}^{2+}$-assisted ER $\left(\mathrm{Ca}^{2+}-\right.$ ER/RER) when PLC $\beta$ serves as a coincidence detector to collate subtreshold $\mathrm{Ca}^{2+}$ entry and metabotropic receptor activation, none of which would be sufficient alone, to readily trigger 2-AG release [298,593,594].

In contrast to the short forms of $\mathrm{CB}_{1} \mathrm{R}$-dependent plasticity that normalize within seconds, lasting activation of the $\mathrm{CB}_{1} \mathrm{R}$ on the minute scale (either via long trains of depolarization or external application of agonists) causes long-term depression ( $\mathrm{CB}_{1} \mathrm{R}$-LTD) of inhibitory and excitatory transmission. Importantly, $\mathrm{CB}_{1} \mathrm{R}$-LTD requires additional presynaptic stimuli to take place, including intracellular $\mathrm{Ca}^{2+}$ concentration rise, or receptor stimulation $[299,582,586]$. Only the induction but not the maintenance of the $C_{1} R$-LTD requires the $C_{1} R$, and once LTD is induced, it involves the following steps: the $G_{\alpha i}$ subunit detaches from the $\mathrm{CB}_{1} \mathrm{R}$, and inhibits adenylyl cyclase, leading to the decrease in protein kinase $\mathrm{A}$ (PKA) activity. In the absence of $\mathrm{CB}_{1} \mathrm{R}$ activation, PKA constantly phosphorylates Rab3-interacting molecule- $1 \alpha$ and $-2 \alpha$ (RIM1/2 $\alpha$ ), thus maintaining the integrity of the synaptic scaffold. An additional stimulus besides $\mathrm{CB}_{1} \mathrm{R}$ activation induces $\left[\mathrm{Ca}^{2+}\right]_{\mathrm{i}}$ rise that activates calcineurin, which in turn dephosphorylates RIM1/2 $\alpha$ in the absence of PKA activity, and consequently destabilizes the release machinery for long minutes or hours [299,582,588,595,596] (Fig. 6). If we take marijuana ingestion as an example, it will also cause lasting stimulation of the $\mathrm{CB}_{1} \mathrm{Rs}$ in the brain. However, it is not entirely clear to us whether lasting presence of $\Delta^{9}$-THC around its receptors is enough to cause LTD in the brain, or the $\mathrm{CB}_{1} \mathrm{Rs}$ would rather trigger $\beta \gamma$ subunit-mediated shortterm plasticity. It is sure though that cannabis decreases transmitter release probability at central synapses; just the exact mechanisms have not yet been fully detailed. Of course, not only 2-AG but other endocannabinoids, e.g., anandamide and NADA are also fully capable of inhibiting depolarization-induced $\mathrm{Ca}^{2+}$ entry in the presynaptic terminals, which we demonstrate for this review in striatal nerve terminals (Fig. 10B1-B5).

\subsubsection{Spike-Timing-Dependent Plasticity}

Spike-Timing-Dependent Plasticity (STDP) is a major synaptic learning rule that integrates the recent pre- and postsynaptic activities on the millisecond scale and adjusts synaptic strength accordingly [597]. STDP is probably a basic mechanism that tailors brain physiology. New exciting physiopharmacological studies combined with mathematical modeling unveiled the crucial role of the $\mathrm{CB}_{1} \mathrm{R}$ at corticostrial STDP $[598,599]$. First, the authors showed that the number and the order of presynaptic and post-synaptic stimulation pairs (within 30 msec interval) can profoundly affect the outcome of synaptic plasticity. 75-100 post-pre pairings induced NMDAR-dependent long-term potentiation (NMDAR-LTP), which was mimicked with 2-AG puffs [598]. This was later shown to be $\mathrm{Ca}^{2+} /$ calmodulindependent protein kinase II (CaMKII)-dependent (the amount of activated CaMKII progressively accumulates with the number of pairings) [599].

Surprisingly, a low number, i.e., 5-10 post-pre pairings also induced LTP at $1 \mathrm{~Hz}$, which was dependent on mGlur5, muscarinic $M_{1}$ receptor $\left(M_{1} R s\right), D_{2} R$, TRPV $V_{1} R, G_{q / 11}, P L C \beta$, 
DAGL $\alpha$, 2-AG release and $\mathrm{CB}_{1} \mathrm{R}$ activation $\left(\mathrm{CB}_{1} \mathrm{R}-\mathrm{LTP}\right)$. It can be induced with few pairings in both striatopallidal and striatonigral medium spiny neurons (MSNs). $\mathrm{GABA}_{\mathrm{A}}$ receptors set the polarity: picrotoxin reverses the post-pre into pre-post requirements. At higher frequencies, symmetric Hebbian plasticity was observed, because post-pre pairing also caused LTP with mixed $\mathrm{CB}_{1} \mathrm{R}$-NMDAR-dependent phenotype [598].

Finally, the authors found that 50-100 pre-post pairings induced $\mathrm{CB}_{1} \mathrm{R}$-dependent LTD, but below 50 pre-post pairs, no LTD was observed. To reconcile the complex results, the authors used mathematical models which closely described the phenomena: Prolonged release of moderate to high levels of 2-AG leads to $\mathrm{CB}_{1} \mathrm{R}-\mathrm{LTD}$ via calcineurin inhibition, while brief releases of high 2-AG concentration yield LTP via $\mathrm{CB}_{1} \mathrm{Rs}$ coupling to PKA activation [599]. Therefore, intermediate levels of $\mathrm{CB}_{1} \mathrm{R}$ activation trigger $\mathrm{CB}_{1} \mathrm{R}$-LTD through a combination of PKA inhibition and calcineurin activity, whereas high levels of $\mathrm{CB}_{1} \mathrm{R}$ activation lead to $\mathrm{CB}_{1} \mathrm{R}$-LTP through the reverse combination: PKA activation combined with calcineurin inhibition. For the rest of the non$\mathrm{CB}_{1} \mathrm{R}$-dependent conditions see [599].

\subsubsection{Post-Synaptic Plasticity}

There are $\mathrm{CB}_{1} \mathrm{Rs}$ also in somatodendritic (i.e., postsynaptic) compartments with the ability of controlling synaptic plasticity, as reported in cortical pyramidal cells [600] and GABAergic interneurons [601]. The activation of these post-synaptic $\mathrm{CB}_{1}$ Rs causes slow self-inhibition, which is another form of synaptic plasticity probably relevant for the understanding of the effects of cannabis. We also found $\mathrm{CB}_{1}$ Rs post-synaptically in the dorsal striatum [390] as well as in the rodent and human cerebral cortex and hippocampus (Köfalvi, unpublished data). In the hippocampus, a new publication showed that $\mathrm{CB}_{1} \mathrm{Rs}$ in the dendrites of pyramidal cells close to the stratum radiatum in the dorsal hippocampus of mice control hyperpolarisation-activated, cyclic nucleotide-gated (HCN) channels [602]. HCN channels are cation channels that are activated by hyperpolarisation at negative voltages around $-50 \mathrm{mV}$ [603]. The cyclic nucleotides cAMP and cyclic guanosine monophosphate (cGMP) directly enhance channel activity, underlying pacemaker currents found in many excitable cells including neurons [603]. HCN channels modulate dendritic integration of excitatory inputs, learning and memory [604,605]. Maroso and colleagues [602] found that the activation of local dendritic $\mathrm{CB}_{1} \mathrm{Rs}$ substantially increased the depolarizing sag response, a measure of HCN activity under somatic wholecell current clamp mode. The authors elegantly unvelied the steps leading to HCN current modulation, which started with 2-AG activation of the $\mathrm{CB}_{1} \mathrm{R}$, leading to the stimulation of mitogen-activated protein kinases (MAPKs) of the JNK family, which in turn can boost cGMP levels through increased activation of nitric oxide-mediated activation of guanylyl cyclase. As mentioned above, the increase in cGMP levels consequently triggers a depolarizing shift in the activation curve of the HCN1 channel. To our knowledge, this study is the first presenting clear evidence of postsynaptically located functional $\mathrm{CB}_{1} \mathrm{Rs}$ in the hippocampus. Furthermore, the authors revealed that the activation of this novel $\mathrm{CB}_{1} \mathrm{R}-\mathrm{HCN} 1$ axis can block the induction of long-term potentiation (LTP), evoked by theta-burst stimulation of either the Schaffer-collaterals or the temporoammonic pathway. Consequently, $\mathrm{CB}_{1} \mathrm{R}$ activation via $\mathrm{HCN} 1$ channel modulation can also impair spatial memory consolidation [602].

\subsubsection{The Therapeutic Potential of Cannabinoids}

The therapeutic potential of cannabis and cannabinoids has been recently assessed by a meta-analysis of randomized clinical trials on the following health conditions: nausea and vomiting due to chemotherapy, appetite stimulation in HIV/AIDS, chronic pain, spasticity due to multiple sclerosis or paraplegia, depression, anxiety disorder, sleep disorder, psychosis, intraocular pressure in glaucoma, or Tourette syndrome [606]. The authors also reviewed case series and other studies based on eligibility. The evaluated cannabinoids were synthetic forms of $\Delta^{9}$-THC or CBD alone, $\Delta^{9}$-THC combined with CBD (Nabiximols, Sativex $\left.{ }^{\circledR}\right)$, or the two synthetic $\Delta^{9}$-THC-analogs, levonantradol and nabilone per se. The authors [606] concluded that there was moderate-quality evidence to support the use in chronic neuropathic or cancer pain (smoked $\Delta^{9}$-THC and nabiximols) and spasticity due to multiple sclerosis (nabiximols, nabilone, $\Delta^{9}$-THC/CBD capsules and dronabinol). For the rest of the conditions, they found limited statistical support for therapeutic potential; however, phytocannabinoids were associated with an increased risk of adverse effects [606]. The negative overall result of the metaanalysis is probably due to inadequate design of the trials rather than a lack of therapeutic potential for phytocannabinoids [607]. Indeed, there is an increasing need to support research on cannabis and phytocannabinoids because the legalization of cannabis and medical marijuana worldwide meets controversial reception by the societies, researchers and health care professionals [607]. For instance, acclaimed Canadian researchers point out the discrepant policy of Canada which supports cannabis use for medical purposes but has little interest in financing cannabis research [608]. They emphasize that the state of Colorado, US, harnesses private support from the medical marijuana industry to support cannabis research. This is in fact homologous to those private funds from the tobacco and coffee industries that support research on nicotine and caffeine.

Phytocannabinoids of the cannabis plant have been proven therapeutic potential in a number of human disorders, and these molecules also serve as useful templates for organic chemists to design novel medicines [21,609]. Below we review the physiopharmacology of the endocannabinoid system and the therapeutic potential of cannabis in selected health conditions. Unfortunately, due to space constraints, we can only refer to other excellent reviews and original works for additional (patho)physiological cases, including but not limited to analgesia, inflammation and pain [610612], immunomodulation and beyond [613-615]; neuroinflammation and neurodegeneration [23,616-618], stroke and subacute brain trauma [619-621], multiple sclerosis and spasticity [622-624], normal and pathological brain aging [618,625], suicide [626,627], liver diseases and hepatic encephalopathy [628-630], food intake and eating disorders [631-634], systemic energy balance and metabolic disorders [260-262,490,635-637], mitochondrial function [458,638-641], cancer and cancer care [642-646]; osteoarthritis [647,648]; bone remodelling, bone metabolism and osteoporosis [490,566,649-652], asthma, bronchospasm, 
respiratory tract changes [249,653], glaucoma [654,655], bladder dysfunction [656], preimplantation embryo development and miscarriage $[657,658]$.

\subsubsection{Cannabidiol - a New Adjunctive Therapy for Epileptic Seizures?}

The therapeutic role of cannabinoids in epilepsy and excitotoxicity deserves an additional paragraph [22,659664]. As a result of the three-decade knowledge that CBD lowers seizures for epilepsy, clinical trials with CBD are underway with the objective of marketing this useful molecule as an antiepileptic medicine [21]. Similarly, a recent pediatric clinical trial with medical cannabis oil of high (20:1) CBD: $\Delta^{9}$-THC ratio significantly reduced the severity and incidence of intractable epilepsy in the majority of the 74 children and adolescents [23]. GW Pharmaceuticals has been conducting four Phase III pivotal clinical trials in the US with Epidiolex ${ }^{\circledR}$ (a liquid formulation of pure plantderived $\mathrm{CBD}$ ), as an adjunctive therapy for the treatment of seizures associated with rare and catastrophic forms of childhood-onset epilepsy, namely Dravet syndrome, LennoxGastaut syndrome and tuberous sclerosis complex. On March 14, 2016, GWPharma announced positive results from the first of these pivotal trials in Dravet syndrome. This trial enrolled 61 Epidiolex ${ }^{\circledR}$ - and 59 placebo-treated children (mean age: 10 years), who have been already on average on their $5^{\text {th }}$ type of antiepileptic medication and still had 13 convulsive seizures per month (median baseline frequency). Epidiolex ${ }^{\circledR}(20 \mathrm{mg} / \mathrm{kg} /$ day) caused a significant $39 \%$ median reduction $(\mathrm{P}<0.01)$ in monthly convulsive seizures, which was 3-times greater than the placebo effect [665].

\subsubsection{Modulation of Neuromodulation}

Cannabinoids not only affect the activity of the principal (glutamatergic) cells and the GABAergic inhibitory neurons, but are also capable of modulating the release of other neuromodulators. This interaction is bidirectional, because endocannabinoid release can be triggered by the stimulation of neuromodulator receptors (Figs. 6 and 8). Finally, cannabinoid receptors can form heterodimers with receptors of other neuromodulator systems (Fig. 6). Such complex interaction with other neuromodulators at different levels provides the basis for the fine-tuning of synaptic transmission and synaptic plasticity, and lie behind learning and memory, emotions, stress coping, mood, motivation, reward, and cognition among others [666,667]. The delicate spatiotemporal regulation of this system allows an exceptional flexibility in the control of brain functions. Chronic intake of exogenous cannabinoids in the form of either agonists such as $\Delta^{9}$-THC or inverse agonists such as SR141716A (rimonabant, Acomplia ${ }^{\mathrm{TM}}$ ) takes away this flexibility. Rigid neuromodulation cannot correctly regulate neural plasticity, and the patient may drift into neuropsychiatric conditions. These include the typical chronic mental consequences of regular cannabis intake [668-670], tolerance such as hyperemesis [671] and cannabis withdrawal symptoms [672], as well as Acomplia ${ }^{\mathrm{TM}}$-induced depression and suicide [263,673].

\subsubsection{The Interaction of Cannabis with Dopamine in the Reward Areas}

Drug-induced increases in midbrain dopaminergic activity or striatal/accumbal dopamine levels or both predict an abuse potential for the substance [674-676].
Cannabimimetics including $\Delta^{9}$-THC increase midbrain dopaminergic activity or extracellular dopamine levels in the striatum or both of laboratory animals [677-686]. Inhaled cannabis also increases the displacement of the $D_{2} / D_{3}$ receptor tracer $\left[{ }^{11} \mathrm{C}\right]$ raclopride in the ventral striatum and the precommissural dorsal putamen of human individuals [687], i.e., in the reward areas associated with the subjective "high" and the abuse liability of cannabis. The underlying mechanism for cannabinoid-stimulated dopamine increase is thought to be indirect: midbrain dopaminergic cell clusters are under tonic inhibition by the dense GABAergic innervations arising from the tail of the ventral tegmental area (VTA)/rostromedial tegmental nucleus and from other areas. The activation of presynaptic $\mathrm{CB}_{1} \mathrm{Rs}$ disinhibits these GABAergic synapses, thus resulting in the increase of the firing activity of the dopaminergic target cells [688-690].

In contrast, there is a high probability that $\mathrm{CB}_{1} \mathrm{Rs}$ do not exert a major role (if any) in the presynaptic modulation of dopamine release in the basal ganglia, albeit this question is not satisfactorily settled. Some found a virtual absence of $\mathrm{CB}_{1} \mathrm{R}$ mRNA [691] and $\mathrm{CB}_{1} \mathrm{R}$ protein [552] in the dopaminergic nerve terminals of the striatum. However, other studies did find sparsely distributed $\mathrm{CB}_{1} \mathrm{R}$ mRNA in dopaminergic cells [335] and $\mathrm{CB}_{1} \mathrm{R}$ protein in dopaminetransporter-positive nerve terminals at low levels [390]. We speculate that $\mathrm{CB}_{1} \mathrm{R}$ mRNA is likely present in many mammalian cells which have mitochondria $[458,459,639,640]$, because the cell nucleus provides the mRNA for the trafficking of the $\mathrm{CB}_{1} \mathrm{R}$ to the outer leaflet of the mitochondrion. Therefore, the presence of $\mathrm{CB}_{1} \mathrm{R}$ mRNA and protein is insufficient evidence for a direct neuromodulator role of the $\mathrm{CB}_{1} \mathrm{R}$ in a given cell. Moreover, albeit cannabimimetics fail to directly modulate single pulse stimulation-evoked release of dopamine in striatal and accumbal slices [692-694], they can do so on the release of dopamine evoked by a train of pulses in striatal slices [695], possibly by disinhibiting $\mathrm{D}_{2}$ autoreceptor-mediated presynaptic inhibition of dopamine release [694], or by modulating local GABAergic signaling in the striatum [693] and the nucleus accumbens [696]. Stunningly, some of the above studies reported that cannabimimetics inhibited the in vitro release of dopamine [693-695], contrasting other in vitro studies that found no effect [390] or even, detected stimulation [696].

In fact, the vital roles of cannabinoids in dopaminergic modulation, and the decisive role of dopamine in reinforcement learning and motivation for incentive stimuli underlines the central role of the endocannabinoid system in reward and addiction [697-699] (see also Section 3.3.5.5). This statement is strongly corroborated by the following observations: $\mathrm{CB}_{1} \mathrm{R}$ activation is a prerequisite for the excitation of mesoaccumbal dopaminergic pathway by nicotine, ethanol and cocaine [685]; mice mutant for major genes of the endocannabinoid system exhibit impaired addictive behavior [700,701]; cannabimimetics reinstate extinguished drug-seeking behavior after a prolonged withdrawal [702-704]; and thus $\mathrm{CB}_{1} \mathrm{R}$ antagonists show great promise in controlling addiction [705-707]. However, the feasibility of addiction therapies based on $\mathrm{CB}_{1} \mathrm{R}$ blockers is tapered by the significant side effects caused by inverse agonism [263,673]. The workaround solution could be the use of silent $\mathrm{CB}_{1} \mathrm{R}$ antagonists [232,395], but the allosteric modulation of the $\mathrm{CB}_{1} \mathrm{R}$ also holds promise [406,708]. 
Recently, $\mathrm{CB}_{2} \mathrm{R}$ ligands have attracted increased interest for the treatment of drug dependence [344,345,709]. Indeed, $\mathrm{CB}_{2} \mathrm{Rs}$ are present in both the nucleus accumbens [710] and midbrain dopaminergic cells, and JWH133 efficiently decreased cocaine self-administration in rats and mice $[344,345]$. Last but not least, there is one question left to be answered: Is cannabis is a gateway drug, that is, can cannabis use increase the probability of transition to "heavier" drugs? Even though many healthcare professionals and researchers would respond with yes to this relevant question, to give a statistically sound answer is almost impossible [711-713]. A recent review proposes a dual hypothesis for the likely association of earlier experience with marijuana with following later use of heavier drugs: (1) based on animal models, it is possible that early marijuana use causes dopaminergic dysfunction in the reward area which increases the likeliness of moving towards heavy drugs, or (2) increased individual susceptibility to addiction first manifests in the abuse of the easily accessible marijuana, and later of the harder substances [177]. In fact, decreased striatal dopamine release has been found in cannabis dependent patients as compared with controls [714].

Below we will discuss an additional level of interaction between the two neuromodulator systems, namely that $D_{2} R$ activation increases endocannabinoid tone in the brain $[715,716]$. Yet another unexpected form of cannabinoiddopamine interaction is worth mentioning: in the low micromolar range, cannabinoid receptor agonists and antagonists including $\mathrm{CBD}$ can inhibit the dopamine transporter (DAT) of the rat and the mouse, independently from cannabinoid receptors [717-719]. This cross-sensitivity between DAT and cannabinoids is bidirectional, because many DAT inhibitors including cocaine is a PAM at the $\mathrm{CB}_{1} \mathrm{R}$ [407]. This means that cocaine, besides directly increasing accumbal dopamine levels, also stimulates $\mathrm{CB}_{1} \mathrm{R}$ signaling, reinforcing its actual addictive nature (see also Section 3.2.3.1 and [406]).

\subsubsection{Cannabinoid Interaction with Acetylcholine}

Cholinergic signaling has robust roles in cognition, metabolism, immunity, and is implicated in the etiology of neuropsychiatric disorders such as Alzheimer's disease and addiction [720]. Data suggest diverse cholinergic responses to cannabinoids varying among brain regions, species and doses. In vitro, WIN55212-2 strongly and potently inhibited the evoked release of acetylcholine in the hippocampus and the neocortex [721-724] - but not the striatum or the nucleus accumbens of rats and mice [695,721,723]. For the sake of this review, we now also tested WIN55212-2 on the electrically evoked release of acetylcholine from superfused striatal slices of the rat, and we can confirm the lack of cannabinoid effect on striatal acetylcholine release (Fig. 10A1-A2). In human neocortical slices, WIN55212-2 produced a biphasic concentration-response curve on the electrically evoked release of acetylcholine, and under some stimulation paradigms, SR141716A stimulated thereof, indicating the existence of endogenous control by endocannabinoids on acetylcholine release [724].

In vivo, WIN55212-2, CP55940 and $\Delta^{9}$-THC all reduced acetylcholine levels in microdialysates from the hippocampus and the medial prefrontal cortex of freely moving rats, while SR141716A increased acetylcholine efflux [725,726]. Systemically administered WIN55212-2 at a low dose $(0.5 \mathrm{mg} / \mathrm{kg})$ stimulated hippocampal acetylcholine efflux via the disinhibition of the septum - the origin of the majority of hippocampal cholinergic nerve terminals, whereas at $5 \mathrm{mg} / \mathrm{kg}$ dose, it inhibited acetylcholine release via intrahippocampal $\mathrm{CB}_{1} \mathrm{Rs}$ [727]. Soon a follow-up study demonstrated that both systemic and local intrahippocampal infusions of the $\mathrm{CB}_{1} \mathrm{R}$-preferring antagonists, SR141716A and AM251 readily and dosedependently increased hippocampal acetylcholine release [580]. This study also identified dopamine-mediated and direct actions of $\mathrm{CB}_{1} \mathrm{Rs}$ in hippocampal acetylcholine release, and reported high percentages of $\mathrm{CB}_{1} \mathrm{R}$-positive cholinergic and dopaminergic nerve terminals in the rat hippocampus. This is in agreement with a previous pioneering paper reporting that one third of septal cholinergic neurons that project to the hippocampus are positive for both $\mathrm{CB}_{1}$ and $\mathrm{GABA}_{\mathrm{A}}$ receptors [728]. Endocannabinoid signaling also has crucial neurodevelopmental role in the fetal pathfinder cholinergic afferents [729]. Outside the hippocampus, however, the presence of $\mathrm{CB}_{1} \mathrm{R}$ immunoreactivity in cholinergic cell bodies and nerve terminals remain controversial in the adult brain $[730,731]$, and for instance the large aspiny cholinergic or somatostatin-positive striatal interneurons are devoid of the receptor [732], in line with the findings of lack of $\mathrm{CB}_{1} \mathrm{R}$ effects on striatal acetylcholine release. These findings implicate predominantly polysynaptic rather than presynaptic roles for $\mathrm{CB}_{1} \mathrm{Rs}$ in controlling cortical acetylcholine release.

Though intrastriatal $\mathrm{CB}_{1} \mathrm{Rs}$ do not appear to modulate striatal acetylcholine levels, an interaction does happen in an inverse fashion: the $M_{1} R$ was shown to elicit 2-AG release in the dendrites of MSNs, which caused retrograde activation of presynaptic $\mathrm{CB}_{1} \mathrm{R}$ in GABAergic synapses, thereby heterosynaptically disinhibiting MSN activity [733]. In fact, the basal ganglia are not the only place where this occurs: it seems to be the rule rather than the exception that acetylcholine, by activating $\mathrm{M}_{1}$ or $\mathrm{M}_{3}$ muscarinic receptors, mobilizes 2-AG, by involving PLC $\beta$ and DAGL $\alpha$, and consequently inhibits GABA release in the hippocampus and the cerebral cortex [734]. This process is a typical example for MSI (see also Section 3.3.2.1), and it is likely that many effects of muscarinic drugs could be blocked by a $\mathrm{CB}_{1} \mathrm{R}$ antagonist.

The first footnote to the above studies is that AM251 and SR141716A could have elicited their stimulatory effects on acetylcholine and monoamines via GPR18 inhibition or GPR55 activation, too (see Sections 3.2.5-6). Nevertheless, WIN55212-2 causes an opposite effect in the above assays without being a ligand either of the GPR18 or of the GPR55. Hence, we stick to the simplest explanation, that is, the effects of cannabinoids on forebrain acetylcholine and monoamine levels are mainly mediated by $\mathrm{CB}_{1}$ Rs.

The second footnote implicates unorthodox interaction between two signaling systems. Many cannabinoids can directly interact with ligand- and voltage gated channels including the $\alpha 7$ nicotinic acetylcholine receptor $(\alpha 7$ $n A C h R)$, the $5-\mathrm{HT}_{3} \mathrm{R}$ serotonin receptor, voltage-gated $\mathrm{Ca}^{2+}$, $\mathrm{Na}^{+}$and $\mathrm{K}^{+}$channels and the NMDA receptor, respectively $[369,381,509,735,736]$. Cannabis constituents are not thought to have major off-target effects on the above 
channels at reasonable concentrations except CBD which exhibits a moderate antagonist activity at the human $\alpha 7$ nAChRs [737]. Remarkably, an elegant study showed that the behavioral consequences of $\Delta^{9}$-THC in squirrel monkeys and rats, including dependence and $\Delta^{9}$-THC-triggered dopamine release in the reward center are counteracted by a kynurenine 3-monooxygenase inhibitor [738]. The authors identify the $\alpha 7 \mathrm{nAChR}$ as the target of KynA. Hence, CBD may be able to counteract some $\Delta^{9}$-THC effects at the $\alpha 7$ nAChR regarding the importance of the $\alpha 7 \mathrm{nAChR}$ in marijuana addiction [738]. CBD has important antipsychotic potential [217], but some of the symptoms of schizophrenia, namely, deficits in cognition, sensorimotor gating and voluntary smooth pursuit eye movement deficit are reliant upon impaired $\alpha 7 \mathrm{nAChR}$ signaling [739-742]. While $\alpha 7$ nAChR activation would likely help to alleviate these negative symptoms of schizophrenia [217], it is worth to mention that $\alpha 7 \mathrm{nAChR}$ activation in neurons also stimulates anandamide formation [743]. An increase in anandamide levels is thought to worsen the positive symptoms of schizophrenia [217]. It is therefore an open question whether the antipsychotic efficacy of CBD is in part reliant on $\alpha 7$ nAChR blockade and consequent decrease in anandamide production, or the other way around, if CBD actually worsens some negative symptoms of schizophrenia by blocking $\alpha 7$ nAChRs.

\subsubsection{Cannabinoid Interaction with Serotonin and} Noradrenaline

The apparent discrepancy between cortical and subcortical cannabinoid modulations also exists for monoamines: systemic SR141716A administration facilitates the release of serotonin, dopamine and noradrenaline in the prefrontal cortex [744,745] whereas in the nucleus accumbens, only serotonin is stimulated by $\mathrm{CB}_{1} \mathrm{R}$ blockade $[744,746]$. This assumes that functional cannabinoid receptors are present in cortical and hippocampal monoaminergic nerve terminals. Indeed, $\mathrm{CB}_{1} \mathrm{Rs}$ are present in the raphe neurons that give rise to forebrain serotonin innervation [747] as well as in frontocortical serotonergic nerve terminals [396]. $\mathrm{CB}_{1} \mathrm{Rs}$ are also present in noradrenergic varicose terminals of the frontal cortex $[748,749]$. The source of the endocannabinoid 2-AG to activate monoaminergic fibres in the frontal cortex is likely the cortical neurons [750], thus retrograde endocannabinoid control is also relevant for monoamines.

$\mathrm{CB}_{1} \mathrm{R}$ activation inhibits the electrically and chemically evoked release of serotonin and noradrenaline in the cerebral cortex including the frontal cortex and the hippocampus $[396,722,751,752]$. The stimulated release of serotonin in $\mathrm{CB}_{1} \mathrm{R}$-/- frontocortical nerve terminals is greater compared to WT nerve terminals [396]. In mice, $\Delta^{9}$-THC $(2.5 \mathrm{mg} / \mathrm{kg})$ as well as CBC and CBD, but not $\Delta^{8}$-THC, CBG or CBN, acutely elicited antidepressive behavior in the forced swim test or the tail suspension test or both [753]. However, chronic cannabis intake is associated with an increased risk for depression - an affective disorder thought to involve monoaminergic dysregulation [668,669]. For instance, in adult rats, chronic (12-day) treatment with HU210 caused an imbalance in $5-\mathrm{HT}_{1 \mathrm{~A}} \mathrm{R}$ vs. $5-\mathrm{HT}_{2 \mathrm{~A}} \mathrm{R}$ activity [754]. Also in adult rats, chronic (8-day) treatment with $\Delta^{9}$-THC reduced frontocortical 5-hydroxyindolacetic acid vs. serotonin levels, indicating decreased serotonergic activity [755].
Interestingly, the increase in serotonin $5-\mathrm{HT}_{2 \mathrm{~A}}$ receptor (5$\mathrm{HT}_{2 \mathrm{~A}} \mathrm{R}$ ) sensitivity in the prefrontal cortex seen by the previous study [754] is apparently $\mathrm{CB}_{2} \mathrm{R}$-dependent: repeated administration of CP55940 increases $\mathrm{CB}_{2} \mathrm{R}$ mediated arrestin3 recruitment and GRK5 activation, and consequently augments $5-\mathrm{HT}_{2 \mathrm{~A}} \mathrm{R}$ density, conferring susceptibility to anxiety and psychosis in rats [756,757]. Besides regulating mood, sociability and anxiety in rodents [758-761], prefrontocortical $\mathrm{CB}_{1} \mathrm{Rs}$ can also promote active stress-coping behavior in rats via serotonergic mechanisms [762].

Acute $\Delta^{9}$-THC is anxiolytic and antidepressant via $\mathrm{CB}_{1} \mathrm{R}$ - and serotonin $5-\mathrm{HT}_{1 \mathrm{~A}} \mathrm{R}$ dependent mechanisms $[758,759]$. That is why it came as a surprise that the noncannabimimetic CBDA and $\Delta^{9}$-THCV also enhance 5$\mathrm{HT}_{1 \mathrm{~A}} \mathrm{R}$ activation in the nanomolar range, probably acting directly as $5-\mathrm{HT}_{1 \mathrm{~A}} \mathrm{R}$ agonists, hence showing therapeutic potential in the treatment of nausea and schizophrenia [763,764]. Chronologically speaking, CBD was reported first to activate the rat, shrew and human $5-\mathrm{HT}_{1 \mathrm{~A}} \mathrm{R}$ thus exhibiting antiemetic, antidepressive and anxiolytic profile, but its useful concentration ranges and doses were rather high to be therapeutically relevant [765-767]. CBD therefore has the capacity to counteract catalepsy induced by different treatments, including the activation of $\mathrm{CB}_{1} \mathrm{R}$ or the inhibition of NO synthase, the $5-H_{1 A} R$ or the $D_{2} R$, which was demonstrated in a mouse study [768]. CBD via $5-\mathrm{HT}_{1 \mathrm{~A}} \mathrm{R}$ activation has been also shown to mitigate neuropathic pain elicited by the anticancer agent, paclitaxel [769]. Additionally in rodents, CBD acting via $5-\mathrm{HT}_{1 \mathrm{~A}} \mathrm{R}$ confers anxiolysis [766], antidepressant effects [770], and neuroprotection in ischemic injury [771-773] and in hepatic encephalopathy [628].

Another very remarkable property of cannabis is its antiemetic profile [774]. Therefore, the legality of medical cannabis has been granted in some countries against chemotherapy-induced nausea and vomiting [775], but skepticism among physicians and the lack of thorough scientific studies do not support the use of cannabis and synthetic cannabinoids as first-line treatment [776]. Cannabinoids can alleviate nausea and vomiting in many circumstances, involving both central and peripheral mechanisms, as well as $\mathrm{CB}_{1}$ and $\mathrm{CB}_{2}$ receptors, but most importantly, by engaging serotonergic signaling $[777,778]$. This scenario can be expanded with the direct inhibition of the 5- $\mathrm{HT}_{3}$ receptor by cannabinoids [381,509] and the activation of $5-\mathrm{HT}_{1 \mathrm{~A}} \mathrm{R}$ by CBD. The antiemetic property of cannabis has become a dogmatic knowledge. Until recently, the general public was unaware of that chronic cannabis consumption can cause a rebound effect, i.e., cyclic vomiting syndrome or hyperemesis without apparent cause and deleterious consequences [671,779,780]. The patients often have difficulties with admitting their severe cannabis dependence, and they use compulsive hot bathing to control nausea [779,781].

Below we will discuss the significance of cannabinoid receptor complexing in a diverse array of patho(physical) processes. Here we start out with the $\mathrm{CB}_{1} \mathrm{R} / 5-\mathrm{HT}_{2 \mathrm{~A}} \mathrm{R}$ heterodimer. The earliest observation in vitro in rat cortical and cerebellar membranes demonstrated that HU210 and oleamide stimulated $\left[{ }^{3} \mathrm{H}\right]$ ketanserin binding to the highaffinity binding site via $\mathrm{CB}_{1} \mathrm{R}$ activation [782]. Reciprocally, 
serotonin modulated SR141716A and WIN55212-2 binding but not CP55940 binding to $\mathrm{CB}_{1} \mathrm{Rs}$ in rat cerebellar membranes [783]. Many years later a novel study proved the existence of the $\mathrm{CB}_{1} \mathrm{R} / 5-\mathrm{HT}_{2 \mathrm{~A}} \mathrm{R}$ heterodimer [784]. The antagonists of each receptor can cause cross-inhibition to prevent the effect of the agonist of the partner receptor, while the co-activation of both receptors can trigger effects ranging from synergism on the inhibition of stimulated cAMP levels to cross-antagonism on arrestin3 recruitment. The genetic deletion of $5-\mathrm{HT}_{2 \mathrm{~A}} \mathrm{R}$ did not affect $\Delta^{9}$-THC-induced hypolocomotion, decrease in body temperature and anxiogenesis at the dose of $3 \mathrm{mg} / \mathrm{kg}$. Remarkably though, 5$\mathrm{HT}_{2 \mathrm{~A}} \mathrm{R}$ deletion prevented the amnesic effect of $3 \mathrm{mg} / \mathrm{kg}$ dose of $\Delta^{9}$-THC in mice, and impaired the $\Delta^{9}$-THC- $(0.3$ $\mathrm{mg} / \mathrm{kg}$ ) induced anxiolysis and increase in social interactions [784].

Besides dopamine acting at $\mathrm{D}_{2} \mathrm{Rs}$ and acetylcholine at $\mathrm{M}_{1 / 3} \mathrm{Rs}$, other neuromodulators such as noradrenaline and serotonin, too, can use endocannabinoids to execute neuromodulation. Recently, it was found that cocaine blocks noradrenaline reuptake in the VTA of rats, and in turn, the excess extracellular noradrenaline activates post-synaptic $\mathrm{G}_{\mathrm{q} / 11}$-coupled $\alpha_{1}$ adrenoceptors, thereby mobilizing 2-AG, which disinhibits GABAergic afferents onto the same dopaminergic cells [785]. Another study found in rats that $\alpha_{1}$ receptor activation in the dorsal raphe (the origin of ascending serotonergic fibres) triggers endocannabinoiddependent LTD at glutamatergic afferents, and that chronic stress impairs this mechanism [786]. As for serotonergic MSI, 5- $\mathrm{HT}_{2} \mathrm{R}$ activation inhibits glutamatergic afferents in the inferior olive via similar retrograde endocannabinoid mechanism [787].

CBG potently stimulates $\left[{ }^{35} \mathrm{~S}\right]-\mathrm{GTP} \gamma \mathrm{S}$ binding to mouse brain membranes already at a concentration as low as $1 \mathrm{nM}$, but this stimulation is reversed into strong inhibition above the concentration of $1 \mu \mathrm{M}$ [788]. Further analysis revealed that $\mathrm{CBG}$ is an ultrapotent $\alpha_{2}$ adrenoceptor agonist $\left(\mathrm{EC}_{50}\right.$, $200 \mathrm{pM}$ ) and a potent 5- $\mathrm{HT}_{1 \mathrm{~A}} \mathrm{R}$ antagonist (IC $50,52 \mathrm{nM}$ ), as well as a low potency $\mathrm{CB}_{1} \mathrm{R}$ antagonist [788]. This is interesting in that sense that in the frontal cortex, presynaptic $\mathrm{CB}_{1} \mathrm{R}$ activation inhibits noradrenaline release from afferents, and noradrenaline $\alpha_{2} \mathrm{R}$ activation desensitizes this action of the $\mathrm{CB}_{1} \mathrm{R}$ (presumably via heterodimer formation or via second messenger systems) [749]. Strikingly, this is mirrored at the post-synaptic level in layer V/VI pyramidal cells, where both acute and chronic treatment with WIN55212-2 impairs the inhibitory action of $\alpha_{2} \mathrm{R}$ activation [789]. This leads us to speculate that the effect of cannabis consumption on executive functions, mood and anxiety may depend on its $\Delta^{9}$-THC/CBG ratio.

\subsubsection{Footnote: Biphasic Cannabinoid Effects}

The above reported biphasic effects for cannabinoids are not uncommon. At low doses, cannabimimetics stimulate the central responses, while at doses $\sim 10$ times higher, inhibition rather than stimulation is observed. Additional reports include the effects of $\Delta^{9}$-THC on hippocampal 2deoxyglucose use [790] and on the modulation of electrically-evoked cortical potentials [791]. Biphasic responses are general consequences of actions at cannabinoid receptors of different sensitivities that can even form heterodimers with each other (see Section 3.3.5.1), biased agonism (see Section 3.2.3.1), and direct local vs. indirect polysynaptic mechanisms.

The simplest example of polysynaptic events is the concurrent modulation of excitatory glutamatergic and inhibitory GABAergic neurotransmissions. The global (external) activation of cerebral $\mathrm{CB}_{1}$ Rs thus causes both the inhibition of excitation and inhibition in the same time. This can lead to opposing effects in the behavior, as observed in "knockout" mice with selective deletion of $\mathrm{CB}_{1} \mathrm{R}$ in cortical glutamatergic or GABAergic neurons [661], as compared to their wild-type littermates in a predominant C57BL/6N background. In these mice, it was observed that the deletion of $\mathrm{CB}_{1}$ Rs in cortical glutamatergic neurons increases passive coping (freezing time) in fear-conditioning protocol using foot-shocks, while the deletion of $\mathrm{CB}_{1} \mathrm{Rs}$ in GABAergic terminals increases active coping behavior (escape attempts and risk assessment) when facing aversive stimuli [792]. Additionally, accumbal $\mathrm{CB}_{1} \mathrm{Rs}$ cause hypophagia through inhibition of GABAergic transmission, while the activation of those cerebral $\mathrm{CB}_{1} \mathrm{Rs}$ that modulate excitatory transmission increases food intake [631].

$\mathrm{CB}_{1} \mathrm{R}$-mediated bidirectional actions are apparent at the level of the circuitry, too: $\mathrm{CB}_{1} \mathrm{R}$ activation in the basal ganglia direct pathway induces thalamocortical hypersynchrony, whereas the activation of $\mathrm{CB}_{1}$ Rs expressed in cortical glutamatergic neurons decreases cortical synchrony and thalamocortical hypersynchrony [793]. Additionally, the activation of $\mathrm{CB}_{1} \mathrm{Rs}$ in cortical glutamatergic neurons decreases the activity of the cortical networks; however, $\mathrm{CB}_{1} \mathrm{R}$ blockade stimulates network activity when basal network activity is low, whereas the opposite is detected when its initial level is high [794].

An elegant paper recently tackled the neurochemical phenotype of $\mathrm{CB}_{1}$ Rs in GABA- vs. glutamatergic terminals, and the authors found that there are more than twice as much $\mathrm{CB}_{1} \mathrm{Rs}$ in GABAergic than in glutamatergic cells in the hippocampus, albeit the efficacy of $\mathrm{CB}_{1}$ Rs to couple with $\mathrm{G}$ proteins is much greater in pyramidal cells (aka in the hippocampus of mice lacking interneuronal $\mathrm{CB}_{1} \mathrm{Rs}$ ) [795]. This is somewhat in accordance with the observations of one of the authors of this review that the $\mathrm{EC}_{50}$ of the synthetic cannabinoid, WIN55212-2 to inhibit the KCl-evoked release of glutamate is $\sim 7 \mathrm{nM}$ in isolated striatal nerve terminals, while this value is $\sim 70 \mathrm{nM}$ for the evoked release of GABA (Köfalvi, unpublished). This would suggest that glutamatergic nerve terminals are more sensitive to cannabinoid agonists, but this assumption is contrasted in a more complex system, in striatal slices where others found that striatal GABAergic neurotransmission is much more sensitive to endocannabinoids and WIN55212-2 than glutamatergic neurotransmission, hence, low-frequency (1 $\mathrm{Hz}$ ) stimulation elicits preferentially LTD [796]. One possible explanation for this discrepancy is that striatal $\mathrm{A}_{2 \mathrm{~A}}$ adenosine receptors may impede $\mathrm{CB}_{1} \mathrm{R}$ function (see Section 3.3.5.3) in the presence of adenosine in a striatal slice or in vivo, while in superfused nerve terminals, ambient adenosine levels around the receptors are negligible.

\subsubsection{Cannabinoid Receptor Heteromers}

A receptor is the smallest functional unit capable of transducing a chemical signal [797]. One receptor can be composed of several equal or different nonfunctional 
subunits, which make a receptor either homomeric or heteromeric [797]. These functional receptors can further assemble into receptor heteromers with physiopharmacology considerably different from that of the individual components [797]. For instance, metabotropic receptors typically signalize in heteromers formed with other metabotropic and ionotropic receptors, including but not limited to receptors for acetylcholine, adenosine, dopamine, glutamate and serotonin [798-800]. Receptor heteromer mosaics can be assembled from two or more different receptors [797,800,801], with striking consequences to the outcome of their signaling [802-804]. Such example is the $\mathrm{A}_{2 \mathrm{~A}} \mathrm{R}-\mathrm{D}_{2} \mathrm{R}-\mathrm{CB}_{1} \mathrm{R}$ heterotrimer, which assembles via triangular electrostatic interactions among the intracellular domains of individual receptors [805]. These domains are rich in arginine as well as phosphorylatable serine and threonine residues [805]. Endogenous ligands can activate intracellular signaling on the basis of "first come, first served", because the binding of an orthosteric agonist to its cognate receptor often render the partner receptor inactive [806]. This cross-desensitization has been described not only for agonists but also, for antagonists, and such is the case for the dopamine $D_{2}\left(D_{2} R\right)$ and adenosine $A_{2 A}$ receptors $\left(A_{2 A} R s\right)$ [806]. Heteromerization thus provides the cells with the capacity of cross-correlation analysis - the power of local computational units - to prioritize modulator signals.

\subsubsection{Heteromerization within the Cannabinoid Receptor Family}

Endocannabinoid receptors are also prone to assemble with each other, often generating functional diversity. The most trivial example is the $\mathrm{CB}_{1} \mathrm{R}-\mathrm{CB}_{1} \mathrm{R}$ homodimer - the way $C_{1}$ Rs are expected to exist in the cell membrane $[807,808]$. The $\mathrm{CB}_{1} \mathrm{R}-\mathrm{CB}_{2} \mathrm{R}$ heterodimer has been also described in both expression systems and in pinealocytes and in situ, in the nucleus accumbens [710]. The $\mathrm{CB}_{1} \mathrm{R}-\mathrm{CB}_{2} \mathrm{R}$ heterodimer shows negative allosteric interaction, because an inverse agonist of one receptor (AM251 or AM630) was capable of antagonizing the selective agonist of the other receptor, while the simultaneous use of two selective agonists, JWH133 and ACEA resulted in a much smaller effect amplitude (on Akt phosphorylation) than the individual effect amplitudes of each drug alone [710]. Additionally, there is a decrease in the density of the $\mathrm{CB}_{1} \mathrm{R}$, the $\mathrm{CB}_{2} \mathrm{R}$ and their heterodimer in the pallidothalamic pathway in experimental Parkinsonism, while L-DOPA treatment disrupts this heterodimer [809].

The $\mathrm{CB}_{2} \mathrm{R}-\mathrm{GPR} 55$ heterodimer has been recently reported by two groups, both in HEK293 [810,811] and human glioma and breast cancer cell lines [811]. Again, the action of the GPR55 agonist LPI was inhibited by both AM630 and HU308, i.e., the $\mathrm{CB}_{2} \mathrm{R}$ agonist and antagonist, respectively, and vice versa, the effect of HU308 was inhibited by LPI and the GPR55 antagonist, HBA [811]. The excellent twist of this latter study was testing $\Delta^{9}$-THC at a wide concentration-range: $\Delta^{9}$-THC at low concentrations first activated the $\mathrm{CB}_{2} \mathrm{R}$, and thus inhibited forskolin-induced CAMP accumulation and stimulated ERK1/2 phosphorylation. At higher concentrations, $\Delta^{9}$-THC begun to activate the GRP55, too, thus started to inhibit its own effect at the $\mathrm{CB}_{2} \mathrm{R}$ [811]. These data highlight that the concentration-response curve of anti-prolific $\Delta^{9}$-THC action is U-shaped: too high or too low concentrations are both ineffective.

Last but not least, the $\mathrm{CB}_{1} \mathrm{R}$ also forms dynamic protein complexes with the GPR55, for instance, in the vascular endothelium [500]. Here the authors tested the dual agonist, anandamide, which also showed cross-inhibition, but this was dependent of external $\mathrm{Ca}^{2+}$-induced recruitment of adapter proteins. Namely, integrin clustering prevented anandamide from inhibiting GPR55-mediated intraendothelial $\mathrm{Ca}^{2+}$ rises via $\mathrm{CB}_{1} \mathrm{R}$ activation [500]. $\mathrm{CB}_{1} \mathrm{R}-$ GPR55 heteromer is also present in the rat and monkey striatum, and it exhibits cross-antagonism on ERK1/2 phosphorylation and stimulation of nuclear factor of activated T-cells (NFAT) [812]. It is of course difficult to predict how cannabis constituents would affect this highly context-dependent heterodimer, because if $\Delta^{9}$-THC activates both receptors it would produce cross-antagonism on their common second messengers. The concentration of ligands is a crucial factor, because one of the two receptors often has greater affinity toward a common non-selective ligand of both receptors. Besides, the simultaneous presence of other ligands such as CBD and $\Delta^{9}-\mathrm{THCV}$ can greatly affect the signaling outcome of a $\mathrm{CB}_{1} \mathrm{R}-\mathrm{GPR} 55$ heterodimer. In the following paragraphs, we will discuss selected cases of $\mathrm{CB}_{1} \mathrm{R}-\mathrm{GPCR}$ heterodimers. Note that other heterodimers also exists: the $\mathrm{CB}_{1} \mathrm{R}$ has been described in functional heteromeric complexes with other receptors such as the angiotensin $\mathrm{AT}_{1}$ receptor [813], as well as the orexin $\mathrm{OX}_{1}$ receptor [814-816]. However, due to space limitations, these interesting receptor interactions will not be discussed.

In fact, these negative crosstalks among the cannabinoid receptor heteromers, irrespective of the use of agonists or antagonists, are in full agreement with data reported for the $A_{2 A} R-D_{2} R$ heterodimer [806]. It seems plausible that when a ligand binds to its cognate receptor and induces a conformational change impacting the conformation of the partner receptor(s), it is largely irrelevant if the ligand in question was an agonist or an inverse agonist, because either way the ligand affects conformation. Nevertheless, it would be interesting to test how a true silent agonist would affect the partner receptor in the heteromer.

\subsubsection{The $C B_{1} R-D_{2} R$ Heterodimer}

While the above examples covered the so far known $\mathrm{CB}_{2} \mathrm{R}$ and GPR55 heterodimers, the $\mathrm{CB}_{1} \mathrm{R}$ has been shown in much more functional complexes formed with a diverse array of receptors. Perhaps the first such identified heterodimer was with the $\mathrm{D}_{2} \mathrm{R}$ [817-820]. The interaction is directly physical (electrostatic) between the C-terminus and the third intracellular loop of the $\mathrm{CB}_{1} \mathrm{R}$ and the third intracellular loop of the $\mathrm{D}_{2} \mathrm{R}$ [805,820]. Although both receptors alone in homodimer signalize with $G_{i / o}$ proteins, their concurrent activation results in a coupling to $G_{s}$ and the stimulation of cAMP accumulation [817-819]. It is believed that concurrent stimulation of the two receptors would induce heterodimer formation [819]. Ultrastructural evidence documents that the two receptors have partially overlapping subcellular distributions in both dendritic and axonal profiles in the nucleus accumbens [821]. As we discussed above, $\mathrm{D}_{2} \mathrm{R}$-like receptor activation in the striatum triggers postsynaptic anandamide formation and release [715] (Fig. 8). Thus, it is possible that anandamide, produced upon $\mathrm{D}_{2} \mathrm{R}$ 
activation can exert a feedback on its release. This is hinted by the study discussed above [694].

\subsubsection{The $A_{2} R-C B_{1} R$ Heterodimer Filtering the Corticostriatal Inputs}

The striatum is also a venue for the highly studied $\mathrm{A}_{2 \mathrm{~A}} \mathrm{R}-\mathrm{CB}_{1} \mathrm{R}$ heterodimer [822-824]. Strong evidence supports that $\mathrm{A}_{2 \mathrm{~A}} \mathrm{R}$ activation dampens presynaptic $\mathrm{CB}_{1} \mathrm{R}$ signaling at rat corticostriatal nerve terminals $[553,571,825]$. Still there is more to it: pre- and post-synaptic location of $\mathrm{CB}_{1}$ and $\mathrm{A}_{2 \mathrm{~A}}$ receptors, their regulation by $\mathrm{D}_{2} \mathrm{Rs}$ and $\mathrm{mGluR}_{5}$ s, their converging intracellular signaling, and the control of endocannabinoid release by these receptors all make it difficult to disentangle the therapeutic value of the $\mathrm{A}_{2 \mathrm{~A}} \mathrm{R}-\mathrm{CB}_{1} \mathrm{R}$ heterodimer in the (palliative) treatments of Parkinson's disease and marijuana addiction (Fig. 8).

Here, we briefly overview the spatiotemporal and molecular orchestration of $\mathrm{A}_{2 \mathrm{~A}} \mathrm{R}-\mathrm{CB}_{1} \mathrm{R}$ interaction at the corticostriatal synapse, which also serves to highlight the complexity of endocannabinoid function in the regulation of network activity. Presynaptically, half of the corticostriatal nerve terminals is equipped with $\mathrm{CB}_{1} \mathrm{Rs}$, and the half of these $\mathrm{CB}_{1} \mathrm{R}^{+}$terminals is also positive for $\mathrm{A}_{2 \mathrm{~A}} \mathrm{R}$ [553] - the adenosine receptor subtype activated by phasic adenosine peaks originated from the extracellular catabolism of ATP co-released with glutamate from excitatory nerve terminals [826]. Presynaptic $\mathrm{CB}_{1} \mathrm{Rs}$ also inhibit ATP release from depolarized striatal nerve terminals [553], suggesting that $\mathrm{CB}_{1} \mathrm{Rs}$ could control the generation of synaptic adenosine, too. In fact, corticostriatal $A_{2 A}$ Rs form mutually inhibitory heterodimers with adenosine $A_{1}$ receptors [827]. The $A_{1}$ subtype is the receptor activated by ambient (low) synaptic levels of adenosine, keeping a brake on glutamatergic noise. Hence, presynaptically, we deal with at least an $A_{1} R-A_{2 A} R$ $\mathrm{CB}_{1} \mathrm{R}$ heterotrimer. Post-synaptically in the MSNs, $\mathrm{A}_{2 \mathrm{~A}} \mathrm{Rs}$ are present predominantly in the indirect pathway that projects to the globus pallidus [828]. Post-synaptic $A_{2 A} R s$ are highly coexpressed with both $\mathrm{D}_{2}$ Rs [828] and mGluR $\mathrm{R}_{5}$, which co-localize with DAGL $\alpha$ and microtubule-associated protein 2 (MAP2) in the perisynaptic border of dendritic spines of the MSNs [552] (Fig. 8). As briefly mentioned in Section 3.2.1.1, the activation of $\mathrm{G}_{\mathrm{q} / 11}$ protein-coupled metabotropic receptors such as the $\mathrm{mGluR}_{5}$ stimulates DAGL $\alpha$-mediated 2-AG release (Fig. 6). mGluR $_{5}$ is typically activated by glutamate spill-over as a result of highfrequency corticostriatal discharge [829], which leads to 2AG release in the synapse [552,830]. $\mathrm{D}_{2}$ Rs have been also reported in MSN dendritic spines to prolong $\mathrm{mGluR}_{5}-$ induced transient 2-AG release [716] (Fig. 8). Post-synaptic $A_{2 A}$ Rs are negatively coupled to both mGluR $R_{5}$ and $D_{2}$ Rs, thus forming dynamic trimeric complexes [801,823]. $\mathrm{A}_{2 \mathrm{~A}} \mathrm{R}$ activation by phasic adenosine levels inhibits post-synaptic metabotropic receptor-induced endocannabinoid formation $[823,831,832]$. Thus, if certain corticostriatal terminals reach sufficient firing frequency (or "saliency" - see e.g., [833]), the consequent retrograde endocannabinoid signaling will no longer affect glutamate release via presynaptic $\mathrm{CB}_{1} \mathrm{Rs}$ as simultaneously high adenosine levels will allow the $A_{2 A} R$ to overrule the $\mathrm{A}_{1} \mathrm{R}$-mediated $[826,827]$ and the $\mathrm{CB}_{1} \mathrm{R}$ mediated inhibition of corticostriatal glutamate release [553,571]. This rescues the most salient nerve terminals. Then what is the fuss about these interactions of many preand post-synaptic receptors? Our model predicts that neuromodulators, adenosine, dopamine and endocannabinoids may interact with surrounding synapses, too, within a confined space to cause heterosynaptic plasticity. Additionally, corticostriatal terminals which do not generate enough adenosine may be silenced, thus enhancing the saliency of the passing information. When this delicate system permanently loses one of its components the consequences are motor diseases or addiction, as the following two examples demonstrate.

Nevertheless, $\mathrm{CB}_{1}$ Rs are also present in the MSN of both the direct and the indirect pathway, and in the latter, $\mathrm{A}_{2 \mathrm{~A}}$ Rs may form heterodimers with $\mathrm{CB}_{1}$ Rs $[822,834]$. Postsynaptic $A_{2 A} R s$, however, were shown to enhance the action of $\mathrm{CB}_{1} \mathrm{Rs}$ at the corticostriatal terminal, because the selective genetic eliminiation of $\mathrm{A}_{2 \mathrm{~A}} \mathrm{Rs}$ from the MSN impaired the action of WIN55212 on the field potential and the pairedpulse facilitation [835]. The same group later elegantly showed how intricate this dichotomy is between pre- and post-synaptic $A_{2 A} R s$ : in rats overexpressing $A_{2 A} R s$ in cortical but not striatal neurons (i.e., are expected to abundantly express presynaptic $\mathrm{A}_{2 \mathrm{~A}} \mathrm{Rs}$ at corticostriatal terminals), $\mathrm{CB}_{1} \mathrm{R}$ function became impaired both in vivo and in vitro [836]. Certainly, there is more effort required to disentangle the interaction among pre- and posst-synaptic $\mathrm{A}_{2 \mathrm{~A}} \mathrm{Rs}$ and $\mathrm{CB}_{1} \mathrm{Rs}$ in the regulation of striatal information flow [834].

\subsubsection{4. $C B_{1} R$ and $A_{2 A} R$ Ligands as Palliative Therapy in Parkinson's Disease?}

The above-mentioned fine-tuned system can be thrown out of balance by the lack of dopamine in Parkinson's disease (PD) which precludes proper synaptic plasiticity at corticostriatal synapses, leading to dyskinesias $[831,837,838]$. There has been an increasing interest in the therapeutic use of cannabis in basal ganglia disorders to provide symptom relief or to slow the progression of the disease or both $[839,840]$. PD patients report beneficial effects of cannabis use on their symptoms [841,842]. Accordingly, the $\Delta^{9}$-THC analog, nabilone was found to decrease and delay the development of levodopa-induced dyskinesia in both non-human primates [843] and in human patients in a randomized, double-blind, placebo-controlled, crossover trial [844]. In contrast, some admit that the picture could be more nuanced [845]: the administration of phytocannabinoid agonists did not ameliorate dyskinesia and Parkinsonism syndromes in a randomized, double-blind, placebo-controlled crossover trial [846]. However, we believe that controlling the increased adenosine levels in the PD-affected striatum may result in better outcomes. Indeed, $A_{2 A} R$ antagonists mirror the motor effects of $D_{2} R$ agonists, and the overactivation of striatal $\mathrm{A}_{2 \mathrm{~A}} \mathrm{Rs}$ in PD contributes to motor symptoms [847]. The idea of using $\mathrm{A}_{2 \mathrm{~A}} \mathrm{R}$ antagonists (e.g., istradefylline) to delay the onset and mitigate the severity of L-DOPA-induced dyskinesia has been toyed for decades [847]. Thus, we foresee that a clinical trial addressing the beneficial effects of cannabimimetics under istradefylline adjunct therapy could provide a more consistent and probably more successful outcome. Also, the outcomes of clinical trials should be controlled for coffee consumption of the patients as caffeine is a general adenosine receptor antagonist [847].

In fact, the self-report studies admittedly carry some bias because those who regularly use cannabis to treat PD symptoms also get psychological relief from the anxiolytic 
and antidepressant effects of cannabis [310,848], besides, many patients refuse to respond queries [842]. As discussed above, long-term $\Delta^{9}$-THC-treatment or cannabis intake triggers $\mathrm{CB}_{1} \mathrm{R}$ desensitization and down-regulation in the brain [229,451,772]. Nevertheless, $\mathrm{CB}_{1} \mathrm{Rs}$ in the basal ganglia are quite resistant to chronic cannabis intake as shown in 30 male participants with the help of positron emission tomography (PET) using an inverse agonist radioligand of the $\left.\mathrm{CB}_{1} \mathrm{R},{ }^{18} \mathrm{~F}\right] \mathrm{FMPEP}-d_{2}$ [849]. This may contribute to the lack of tolerance to the euphoric effects of cannabis [216]. We also explained earlier that $\Delta^{9}$-THC is a weak agonist and its prolonged presence at low levels around central receptors may cause $\mathrm{CB}_{1} \mathrm{R}$ antagonism. In line with these, animal studies evidence that $\mathrm{CB}_{1} \mathrm{R}$ blockade could surpass the efficacy of cannabimimetics to control motor symptoms in PD $[837,840,850]$. As a matter of fact, the only test carried out in human PD patients [851] did not find improvement with the use of SR141716A (rimonabant). The patients of this study were well-responders to classic dopamine-replacement therapies, thus it would be interesting to involve poorer responders as well. Also, the use of a relatively low SR141716A dose could balance between decreased somatodendritic $\mathrm{CB}_{1} \mathrm{R}$ internalization versus $\mathrm{CB}_{1} \mathrm{R}$ blockade (see Section 3.2.4). Besides, it would be helpful to evaluate neutral antagonists such as NESS0327 on dyskinesias in animal models and in human patients. Last but not least, L-DOPA treatment in itself is a confounding factor that disrupts both $\mathrm{CB}_{1} \mathrm{R}-\mathrm{CB}_{2} \mathrm{R}$ and $\mathrm{A}_{2 \mathrm{~A}} \mathrm{R}-\mathrm{D}_{2} \mathrm{R}-\mathrm{CB}_{1} \mathrm{R}$ heteromers in the basal ganglia in experimental Parkinsonism [812,852]. In conclusion, one cannot directly extrapolate from physiological cannabis actions in the healthy basal ganglia to the potential of cannabis-based medicine in PD.

\subsubsection{The $A_{2 A} R-C B_{1} R$ Heterodimer and $\Delta^{9}$-THC-addiction}

Squirrel monkeys self-administrate $\Delta^{9}$-THC, anandamide and $R$-methanandamide intravenously [853], which subserves a sufficiently good research model to study human cannabis addiction. In fact, the subsequent two studies from the same research group nicely demonstrated that the blockade of presynaptic $\mathrm{A}_{2 \mathrm{~A}}$ Rs counteract while the blockade of post-synaptic $A_{2 A}$ Rs potentiate the intravenous self-administration of $\Delta^{9}$-THC and anandamide, but leaving cocaine self-administration or food intake unaffected $[854,855]$. The distinction between the pre- and postsynaptic $A_{2 A} R s$ is made possible by their different pharmacological profile [856]. These data cannot be conveniently explained by invoking only one responsible brain area. On the contrary, the complexity of these data testifies that $\Delta^{9}$-THC addiction is a result of multilevel interaction between the cerebral cortex and the basal ganglia, and may involve the concomitant modulation of GABA, glutamate and dopamine. Hence, further studies are required to map the therapeutic potential of different $\mathrm{A}_{2 \mathrm{~A}} \mathrm{R}$ ligands as either an adjunct therapy together with cannabinoids in PD or as a monotherapy in cannabis addiction.

Interestingly, adenosine and adenosinergic compounds also act as antagonists at the $\mathrm{TRPV}_{1} \mathrm{R}$ [857], which is another example for the antagonistic relationship between the endocannabinoid and adenosinergic systems.

\subsubsection{6. $C B_{1} R$ Heteromers with Opioid Receptors}

The endogenous opioid signaling constitutes the other major neuromodulator system involved in drug addiction [858]. Interestingly, there is a strong correlation between the opiatergic and endocannabinoid systems [859-862]. For instance, opioids and cannabimimetics exhibit similar pharmacological effects, including analgesia, hypotension, hypothermia, motor impairment and sedation [863-865]. Moreover, $\Delta^{9}$-THC-induced dopamine release in the shell of the rat nucleus accumbens is counteracted by the $\mu_{1}$ opioid receptor antagonist, naloxazine [681]. Some of these interactions are thus a likely result of physical complexing between opioid and $\mathrm{CB}_{1}$ receptors.

The first reported such heteromer was the $\mu$ opioid receptor $(\mu \mathrm{OPR})-\mathrm{CB}_{1} \mathrm{R}$ complex, at which the agonists WIN55212-2 or HU210 co-applied with morphin or DAMGO (a $\mu$ OPR-selective peptide) showed mutual crossantagonism on $\left[{ }^{35} \mathrm{~S}\right]-\mathrm{GTP} \gamma \mathrm{S}$ and the activation of the $\mathrm{G}_{\mathrm{\alpha i}}-$ Rap-Src-Signal transducer and activator of transcription 3 (Stat3) pathway that leads to neuritogenesis [866]. In another study, the coexpression of the $\mathrm{CB}_{1} \mathrm{R}$ was enough to decrease DAMGO-evoked signaling at the $\mu O P R$, and this was prevented by the inverse agonist SR141716A but not by the neutral antagonist, O-2050 [867]. The recently described $\delta$ opioid receptor $(\delta \mathrm{OPR})-\mathrm{CB}_{1} \mathrm{R}$ heterodimer is abundant in mouse cortical neurons, and in the absence of $\delta \mathrm{OPR}$, basal $\mathrm{CB}_{1} \mathrm{R}$ coupling is enhanced, and the potency of HU210 to stimulate $\left[{ }^{35} \mathrm{~S}\right]-\mathrm{GTP} \gamma \mathrm{S}$ binding increases from ultrapotent $\left(\mathrm{EC}_{50}, 190 \mathrm{pM}\right)$ to hyperpotent $(50 \mathrm{pM})$, together with its potency by $+10 \%$ [803]. In Neuro2A neuroblastoma cell line where endogenous $\mathrm{CB}_{1} \mathrm{R}$ is normally trafficked to endosomes, $\delta \mathrm{OPR}$ expression brought the $\mathrm{CB}_{1} \mathrm{R}$ to the cell surface, though diminished by 3-fold the potency of HU210 to stimulate intracellular pathways. Stimulation of intracellular $\mathrm{CB}_{1} \mathrm{Rs}$ by $\mathrm{HU} 210$ increased apoptosis in Neuro2A cell line, while $\delta$ OPR expression increased cell viability threefold. In contrast, in cortical neurons of wildtype (WT) mice, $\mathrm{CB}_{1} \mathrm{R}$ blockade by AM251 triggered an $80 \%$ cell death which was absent in cortical neurons of SOPR -/- mice [803]. Altogether, this study highlights the importance of $\delta \mathrm{OPR}$ in the $\mathrm{CB}_{1} \mathrm{R}$ control of cell fate, with consequences to neurodegenerative disorders and brain cancer. More, $\mathrm{CB}_{1} \mathrm{Rs}$ can also reciprocally control the function of $\delta O P R s:$ in peripheral nerve-lesioned rats, changes in the neocortical density and signaling of both receptors were observed, and a non-signaling concentration of HU210 (1 pM) strongly increased the efficacy of $\delta \mathrm{OPR}$ signaling [868], suggesting that this strategy can be helpful as a palliative treatment in neuropathic pain. Last but not least, pharmacological data prompt the existence of an intestinal $\quad \kappa$-opioid receptor( $(\mathrm{OPR})-\mathrm{CB}_{1} \mathrm{R}-\mathrm{CB}_{2} \mathrm{R}$ heterotrimer, which is activated by the diterpene salvinorin A from Salvia divinorum, resulting in decreases in colonic motility and in neurogenic ion transport [869].

Nonetheless, those who engage in the study of cannabinoid-opioid interactions should be advised that SR141716A and AM251 are potent antagonists/inverse agonists of the $\mu \mathrm{OPR}[389,870,871]$, the $\delta \mathrm{OPR}$ [872] and the

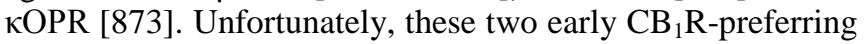
inverse agonists proved to be really "dirty drugs" in the last two decades and they should be replaced with more selective and silent antagonists, such as NESS0327. 


\subsubsection{Additional $C B_{1} R$ Heterodimers and Cell Fate - Receptor Tyrosine Kinases}

The $\mathrm{CB}_{1} \mathrm{R}$ has been found to control the signaling of a diverse array of growth hormone receptors including receptor tyrosine kinases (RTKs) for insulin and insulin-like growth hormone, epidermal growth factor (EGFR); plateletderived growth factor; fibroblast growth factor (FGFR), brain-derived neurotrophic factor (TrkB) and vascular endothelial growth factor in different cellular models such as CHO cells, murine N18TG2 neuronal cells; pancreatic $\beta$ cells, in glioblastoma and lung cancer cells, and neurons of the immature brain [351,874-878]. At the current level of evidence, $\mathrm{CB}_{1}$ Rs most probably control RTKs via adapter proteins or converging intracellular signaling. Nevertheless, it has been shown that $\mathrm{CB}_{1} \mathrm{Rs}$ form heteromeric complexes in the presence of agonists with the TrkB [879] and the insulin receptor $[878,880]$. Whether these complexes involve "bridges" of intracellular or intramembranal proteins or the two receptors are directly connected to each other, is unclear.

Accordingly, cannabimimetics including $\Delta^{9}$-THC have the capability of accelerating cancer growth via the transactivation of EGFR [874] or compromising pancreatic $\beta$ cell viability via the inhibition of insulin signaling [878]. Chronic activation of central $\mathrm{CB}_{1} \mathrm{Rs}$ also disrupts neuritogenesis, growth cone steering, synaptogenesis, and the migration and network integration of neurons, by perturbing signaling at FGFR and TrkB among other RTKs $[361,875,876,879,881,882]$. These led to the recognition that the developing brain is very sensitive to the deleterious effects of cannabimimetics, from the in utero life until the end of adolescence [883-889]. Prenatal cannabis exposure can also lead to epileptic phenotype of the offspring due to impaired connectomes and excitability in the developing brain [882,890,891].

As a footnote, interactions between trophic factors and the endocannabinoid systems also occur beyond the RTK$\mathrm{CB}_{1} \mathrm{R}$ heterodimers: $\mathrm{CB}_{1} \mathrm{R}$ activation stimulates insulin release in pancreatic $\beta$-cells [892] while insulin triggers 2AG release onto accumbal glutamatergic terminals, with a consequent LTD on these excitatory afferents [893]. A $\mathrm{CB}_{1} \mathrm{R}$ antibody has been shown by us to immunoprecipitate the insulin receptor $\beta$ chain in accumbal homogenates of the rat, and $\mathrm{CB}_{1} \mathrm{R}$ activation impaired the insulin-stimulated glucose uptake in rat accumbal slices [880] (see also Section 3.3.9).

\subsubsection{The $C_{2} R$-CXCR4 Chemokine Receptor Heterodimer in Tumor Progression}

A fresh sudy reported that CXCR4 can form an induced heterodimer with $\mathrm{CB}_{2} \mathrm{R}$ in human breast and prostate cancer cells, and simultaneous activation of $\mathrm{CB}_{2} \mathrm{Rs}$ and CXCR4 receptors resulted in reduced CXCR4-mediated expression of phosphorylated ERK1/2, and ultimately, reduced cancer cell functions [894].

\subsubsection{Is Cannabis a Friend or a Foe of the Adult Brain?}

Cannabinoids and their receptors confer neuroprotection $[895,896]$, but only when brain cells are challenged with noxious stimuli [897] such as ischemia and infarct [771-773,898,899], disruptors of ion gradients [900], reactive oxygen species (ROS) [640] or toxic proteins like $\beta$ amyloid [901], as evidenced from animal experiments. Cannabinoid neuroprotection involves both receptor- independent chemical and receptor-mediated mechanisms. The simplest direct action is the neutralization of harmful ROS molecules, because $\Delta^{9}$-THC and congeners - even some of those which lack known activity at any receptors can scavenge ROS [772,902,903]. Receptor-dependent mechanisms encompass a $\mathrm{CB}_{1} \mathrm{R}$-mediated inhibition of excitotoxicity [664,903], as well as the antineuroinflammatory roles of the $\mathrm{CB}_{2} \mathrm{R}[904,905]$, taken that neuroinflammation entails progressive neurodegeneration and impaired brain glucose metabolism.

Nevertheless, several lines of evidence demonstrate that cannabis is toxic to the healthy brain [906]. Long-term administration of $\Delta^{9}$-THC (5-times/week for 8 months) decreased neuronal density and increased astrogliosis in the hippocampi of laboratory rodents, and also augmented the levels of corticosteroids which are known to be neurotoxic on the long run ([907]; see also Section 3.3.9). In man, regular cannabis use decreases the grey matter volume in those brain regions which are associated with memory, cognition, and motivational, emotional as well as affective processing, including but not limited to the hippocampus, amygdala and several areas of the cerebral cortex [908-910]. These changes positively correlate with the frequency of cannabis use and negatively with CBD content. Strikingly, two of these studies found increased cerebellar grey matter volume in regular cannabis users [909,910], but the significance of this finding is unclear. Finally, cortisol levels are also augmented upon acute cannabis use both in healthy controls and frequent users [216]. These indicate that either neuronal viability or neurogenesis or both may be affected by cannabis. First, we discuss the possibility of neurotoxicity.

High $\Delta^{9}$-THC concentrations ( $\left.5 \mu \mathrm{M}\right)$ induce the activation of JNK, p53, then Bax (Bcl-2-associated $X$ protein), caspase-3 and Bcl (B-cell lymphoma oncogene), which pathway causes DNA fragmentation and apoptosis in cultured cortical neurons [911,912]. In hippocampal culture, even a short, 15min exposure to $3.5 \mu \mathrm{M} \Delta^{9}$-THC was found to induce $\sim 70 \%$ cell death within a day, but $0.5 \mu \mathrm{M} \Delta^{9}$-THC also caused $\sim 40 \%$ cell death within 6 days [913]. The authors concluded that $\mathrm{CB}_{1} \mathrm{Rs}$ stimulate the mobilization and the subsequent COX-2-dependent metabolism of arachidonic acid, resulting in the production of ROS and other toxic metabolites, which cause DNA fragmentation and neuronal shrinkage. Many years later, a study elaborated on these observations: the authors found that a single $\Delta^{9}$-THC injection $(5 \mathrm{mg} / \mathrm{kg})$ transiently increased COX-2 (rather than COX-1) expression in the mouse hippocampus, and repeated 7-day intraperitoneal injection increased COX-2 levels permanently in wild-type but not $\mathrm{CB}_{1} \mathrm{R}-/-$ mice [914]. This came as surprise to the authors because 2-AG inhibits COX2 expression in the hippocampus via $\mathrm{CB}_{1} \mathrm{R}$ activation, and $\mathrm{G}_{\text {ai1 }}$ stimulation [914,915]. In contrast, as it turned out, $\Delta^{9}$ THC rather stimulated COX-2 expression via a $\beta \gamma$, Akt, ERK, p38 MAPK, and NF- $\mathrm{BB}$ cascade, thus blaming biased agonism for the untoward effects of $\Delta^{9}$-THC. Next, the authors showed that 7-day $\Delta^{9}$-THC-injection hampered the integrity (decreased spine density) and plasticity of synapses in the hippocampus of these mice, with a consequent impairment in spatial and fear memories [914]. Genetic or pharmacological ablation of COX-2 prevented all these negative effects of $\Delta^{9}$-THC. Strikingly, COX-2 manipulation did not jeopardize the neuroprotective effects of $\Delta^{9}$-THC in 
an animal model of $\beta$-amyloidosis. The authors also discovered that $\Delta^{9}$-THC reduces $\beta$-amyloid levels via the upregulation of the endopeptidase neprilysin, which is a novel model of receptor-mediated neuroprotection [914].

Albeit in these above assays, $\Delta^{9}$-THC was tested for toxicity at relatively high doses and concentrations, there is uncertainty about the peak levels of $\Delta^{9}$-THC around its receptors in the brain during cannabis consumption. Our educated guess is that on an average occasion, a smoked full joint should equal a dosage of a $100-500 \mu \mathrm{g} / \mathrm{kg}$ net $\Delta^{9}$-THC intake. Taken the upper end and dividing it with a low estimate of $50 \mathrm{~kg}$ of soft tissue, we obtain a dose of $10 \mu \mathrm{g}$ $\Delta^{9}$-THC/kg, roughly equivalent with the concentration of $\sim 30 \mathrm{nM} / \mathrm{L}$ aqueous solution (regardless of hepatic metabolism and excretion). This is a crude and simplistic estimation of peak $\Delta^{9}$-THC levels calculated with homogenous drug distribution, although we expect the brain to sequester $\Delta^{9}$-THC from the plasma, hence cerebral $\Delta^{9}$ THC levels might be always greater than those in the plasma (see also Section 3.1.2.1). Altogether, in an infrequent marijuana smoker's brain, we do not expect peak levels of $\Delta^{9}$-THC to be greater than $1 \mu \mathrm{mol} / \mathrm{kg}$ wet tissue, not even under heavy cannabis intoxication, albeit the new trend of "dabbing" cannabis wax may transiently result in way greater peak doses than these estimates. Dabbing apparently poses a huge risk of emergent psychosis [185], which is the topic of Section 3.3.8. Nevertheless, our assumptions on the $\Delta^{9}$-THC doses in the brain are of course very limited because a large part of the brain volume is not a dilute aqueous solution but a dense lipophilic mass, rendering the theories of receptor pharmacology completely useless. Another confounding factor is the higher affinity of the human $\mathrm{CB}_{1} \mathrm{R}$ to $\Delta^{9}$-THC, as compared to the rodent $\mathrm{CB}_{1} \mathrm{R}$ [411], suggesting that lower $\Delta^{9}$-THC doses are sufficient to cause neurotoxicity.

As a result of biased agonism, cannabinoids at very low concentrations may stimulate cAMP production [370] (see Section 3.2.3.1), which in turn can entail excitotoxicity. Thus, the next study looked at the leftmost end of the $\Delta^{9}$ THC dose-response curve, and the striking findings were certainly a game-changer [916]. The extreme low dose of 1 $\mu \mathrm{g} / \mathrm{kg} \Delta^{9}$-THC caused an effect opposite to what intoxicating (psychoactive) doses of $\Delta^{9}$-THC would trigger in drug-naïve mice, i.e., it increased body temperature, potentiated responses to noxious stimuli and increased locomotor activity [916]. Hence, this is another good evidence for contrasting biphasic cannabinoid actions at different doses (see Section 3.3.4.4). Three weeks after the single $\Delta^{9}$-THC(or vehicle-) injection, the mice were trained and tested for their escape latencies in the Morris water maze test, and the $\Delta^{9}$-THC-injected mice still underperformed the vehicleinjected control mice. The authors speculated that extreme low doses of $\Delta^{9}$-THC cause lasting neurotoxicity, but paradoxically, the much higher recreational doses of $\Delta^{9}$-THC achieved on occasions of cannabis ingestion probably protect the brain from the toxic effects [916]. We hypothesize that $\Delta^{9}$-THC may accumulate in non-psychoactive (neurotoxic) quantities in individuals under exposure to cannabis smoke. For this reason, it is imperative to carry out human studies to screen passive cannabis smokers for their plasma $\Delta^{9}$-THC levels and their neurocognitive performance.

\subsubsection{Clearing the Smoke on Neurogenesis}

Adult neurogenesis and gliogenesis provide the adult brain with constant supply of new cells that migrate toward and integrate themselves in the circuitry where they are needed. This process has major implications in learning and forgetting, mood disorders and stroke repair [917-919]. Adult neuronal stem cells also contain $\mathrm{CB}_{1}$ Rs [920-923], and cannabimimetics in most cases stimulate the proliferation of neural progenitors in the neurogenic niches $[918,920,922,924]$. Quite remarkably, the $\mathrm{CB}_{1} \mathrm{R}-/-$ mice exhibit a $\sim 30-50 \%$ reduction in new bromodeoxyuridine(BrdU)-labeled cells [526,921]. Since $\mathrm{CB}_{1} \mathrm{R}$ activation readily stimulates astrogliogenesis beyond neurogenesis [921], this reduction in bromodeoxyuridine-labeling in the $\mathrm{CB}_{1} \mathrm{R}$-/- mice was also accompanied with a relative increase in neurogenesis over astrogliogenesis in the $\mathrm{CB}_{1} \mathrm{R}$-deficient mice [921].

Of course, there is no cannabinoid receptor involvement without contrasting data. Other studies in fact found that $\mathrm{CB}_{1} \mathrm{R}$ activation decreases neurogenesis and progenitor cell differentiation [925,926]. In concert with this, 6 weeks of orally fed $\Delta^{9}$-THC have been found to decrease neurogenesis, while CBD slightly increased proliferation, and even more, it strongly stimulated the survival of neuroblasts. However, this latter case could be attributed to $\mathrm{CB}_{1} \mathrm{R}$ desensitization and elimination upon prolonged activation. Besides, in one of the former studies, only anandamide was tested as agonist, hence the involvement of other receptors beyond the $\mathrm{CB}_{1} \mathrm{R}$ is also possible [925]. Indeed, $\mathrm{CB}_{2} \mathrm{Rs}$ also stimulate neurogenesis and migration of neuroblasts from the subventricular zone (SVZ) to the olfactory bulb, in positive correlation with the age of the mice, i.e., the older the animal the greater the stimulation of neurogenesis and migration by $\mathrm{CB}_{2} \mathrm{Rs}$, although the migration was rather inhibited in young mice [927]. This property of the $C_{2} R$ becomes very useful in the treatment of human immunodeficiency virus- (HIV-) induced neurodegeneration, because $\mathrm{CB}_{2} \mathrm{R}$ activation rescues neural progenitors from HIV-1 glycoprotein Gp120-induced apoptosis, and restores their proliferation [928].

\subsubsection{Cannabis and Psychosis}

The role of impaired endocannabinoid signaling in the etiology of schizophrenia has been widely recognized $[217,697,929]$. The association between psychosis and cannabis use is also clear and unequivocally accepted [930932]. However, many confounding factors - age of initiation of marijuana consumption, dose and type(s) of marijuana consumed including its $\Delta^{9}$-THC/CBD ratio, genetic predisposition, social factors, and abuse of other drugs - all make it very difficult to correctly estimate the weight of marijuana abuse in the onset of psychosis and schizophrenia.

First of all, cannabis use is associated with delusionallike experiences [933]. Even the late-onset cannabis use (i.e., in the range of 21-50 years of age) is associated with elevated risk of psychosis, but clearly, if cannabis dependence took place between the age of 12 and 16 years, the chance for delusional-like experiences was almost tenfold greater than in the non-user population [933].

Cannabis user schizophrenia patients experience the onset of the disease earlier with more positive and less negative symptoms, which is often combined with violent behavior and cannabis dependence [934-936]. Schizophrenia 
patients also found cannabis more rewarding than control subjects [936]. It is also observable that schizophrenia patients prefer cannabis of high $\Delta^{9}$-THC/CBD ratio, such as "skunk" which practically lacks CBD. Cannabis use not only speeds up the development of psychotic disorders, but also worsens the course of the illness, causing more frequent hospital visits, and poorer psychosocial functioning [937939].

There are more schizophrenics among cannabis users than in the general population and more cannabis users become schizophrenic in a given time-frame than non-users $[670,930,940]$. However, the causality between cannabis use and schizophrenia has not been settled. Some discrepancies could not be simply ignored, such that the increased frequency of cannabis use in the general population is not generally associated with greater incidence of schizophrenia population-wide [932,941]. The resolution of these discrepancies is that cannabis precipitates, accelerates the onset and worsens psychosis in susceptible individuals with genetic predisposition, but not in the rest of the population [941]. In other words, genetic traits that render the individual susceptible to schizophrenia (or its prodrome neurochemical status) increase the odds for cannabis use [942]. In concert with these, the Diagnostic and Statistical Manual (5 $5^{\text {th }}$ ed.) [943] already lists CUD as a moderately hereditary psychiatric condition [182,672]. It seems therefore convenient to conclude that cannabis abuse just accelerates the onset and worsens the course of psychosis in susceptible young people, but alarming reports suggest that the case is more complex than that. For instance, south London has startlingly high incidence of new schizophrenia cases, a rate that doubled since 1965, and this is likely due to the appearance of highly potent cannabis breeding lines such as "skunk" [944,945]. In fact, a recent study directly blames "skunk"-like cannabis for at least $1 / 4$ of the schizophrenia cases in south London [945], and "high-potency" marijuana has been aggressively and steadily gaining popularity in the last decades [885].

These latter studies leave open the possibility that the low-potency (classical) cannabis preparations would only facilitate the onset of schizophrenia in the presence of genetic and other environmental liabilities; however, cannabis of high $\Delta^{9}$-THC/CBD ratio itself may provoke schizophrenia in the absence of other major risk factors. A meta-analysis of previous studies has clearly concluded the strong association between CUD and psychosis, and was able to set temporal priority for cannabis use - especially during adolescence - as a causal factor for schizophreniform disorders [930].

Likely, the general belief that cannabis is not overtly dangerous lies in the partial agonist properties of $\Delta^{9}$-THC and its co-occurrence with other molecules (CBD, $\Delta^{9}$ THCV) that can dampen the effects of this psychoactive compound [946]. A confounding factor often ignored in animal studies is the use of the full agonist WIN55212-2 in regimens sufficient to produce clear-cut evidence of causality between $\mathrm{CB}_{1} \mathrm{R}$ activation and schizophrenia symptoms $[178,947]$. In these animal models, exposure to WIN55212-2 during their adolescence but not later, causes persistent psychobiological changes in the adult animals. These animals do not have genetic liability to schizophrenia but still, they exhibit some characteristics of the human psychosis patients if they are treated with WIN55212-2 during the critical neurodevelopmental window of adolescence [178]. Synthetic cannabinoids (e.g., "spice" preparations) are also more potent and efficacious than $\Delta^{9}$ THC (more "WIN55212-2-like"), often full agonists at the $\mathrm{CB}_{1} \mathrm{R}[946,948]$, and therefore they can frequently cause life-threatening or fatal accidents [257,885,946,948-950]. The incisive term "spiceophrenia" has also been coined based on a systematic review of 41 spice-related psychopathology studies [951]. This is a clear indication for the increased health risks associated with full agonist cannabimimetics. The forthcoming years will tell if the growing popularity of potent cannabis variations and synthetic full agonists indeed increases the overall incidence of schizophrenia in the general population, or irrespective of their potency and efficacy, all $\mathrm{CB}_{1} \mathrm{R}$ agonist are alike in that sense that they carry the risk of untoward health effects for those with pre-existing risk factors.

But even if only a fraction of the users were susceptible to cannabis-induced psychosis, the rest of the adolescent users will also have to face negative neurodevelopmental and psychobiological consequences [672,952-955]. Hourslong $\mathrm{CB}_{1} \mathrm{R}$ activation by acute cannabis intake overwhelms the delicate neuromodulator interactions and overrides the regulation of lower and higher order brain functions. This provokes a wide variety of recreational cannabis effects which we discussed above. Chronic exposure to cannabinoids may even offset the threshold sensitivity for feed-back and feed-forward cross-regulations among neuromodulators, often culminating in neuropsychiatric illnesses in the vulnerable. These adverse effects on mental health which are not necessarily dependent on neural development encompass impairment in emotional processing, associative learning, cognition, memory performance [697,954,955-958], substance use disorders $[698,701,959]$, mood disorders, anxiety, impaired sociability, and pathological neuroendocrine response to stress [761,954,960-965].

\subsection{9. $\triangle^{9}$-THC and Steroid Hormones}

Steroid hormones interact with the endocannabinoid system, which has significant therapeutic potential. One important feature of glucocorticoid receptors (GcRs) is that they co-localize with DAGL $\alpha$ in post-synaptic neural compartments apposing $\mathrm{CB}_{1} \mathrm{R}$-positive nerve terminals in the brain [397]. GcR activation in the prefrontal cortex triggers endocannabinoid release [966], which in turn downregulates the hypothalamo-pituitary-adrenal (HPA) axis to decrease circulating corticosteroid levels [967,968], facilitate the extinction and impair the retrieval of aversive memories [965,967,969-971]. A similar phenomenon was suspected based on a pharmacological approach in the rat nucleus accumbens: dexamethasone via GcR activation stimulated 2AG production, and consequently, activated $\mathrm{CB}_{1} \mathrm{Rs}$ in heteromeric complexes with insulin receptors, thus impairing insulin-stimulated accumbal glucose uptake [880]. Such mechanism could explain how chronic stress, Cushingsyndrome or chronic glucocorticoid treatment could impair the regulation of food intake or even, cerebral insulin sensitivity.

Albeit acute mild stress is beneficial and increases the fitness of the individual, acute extreme stress may persistently imprint painful (aversive) memories in the brain, 
leading to post-traumatic stress disorder (PTSD), which is a debilitating psychiatric condition lacking efficient therapeutic strategies [972]. It develops after an extremely stressful event, and the symptoms that can be divided into four clusters are eventually associated with the difficulties of forgetting those painful and shocking memories [972]. In animal models, the activation of amygdalar $\mathrm{CB}_{1}$ Rs facilitates while their blockade dampens the retrieval of aversive memories [969]. In contrast, medial habenular $\mathrm{CB}_{1} \mathrm{Rs}$ are critical for the expression of aversive memories [973]. There is a growing body of evidence for the favorable effects of cannabis on various symptoms of PTSD, including stress, insomnia and nightmares [974,975]. Although the long-term use of cannabis to treat PTSD is cautioned by its abuse potential and the above-mentioned deleterious effects on brain functioning - including susceptibility to psychosis and depression -, the promising small-scale studies and selfreports from PTSD patients prompt large-scale randomized clinical trials under controlled conditions. Besides acute extreme stress, chronic mild stress (as well as subclinical Cushing syndrome and long-term corticosterioid treatments) can also negatively affect the brain, in part because of chronic $\mathrm{CB}_{1} \mathrm{R}$ overactivation that hampers synapses, neuroenergetics and neurogenesis [976].

A recent interesting paper revealed that $\Delta^{9}$-THC and other cannabimimetics (WIN55212-2, HU210) stimulate a massive increase in the synthesis of pregnenolone, the inactive precursor of all steroid hormones, in the brain [406]. The authors also found that the $\mathrm{CB}_{1} \mathrm{R}$ PAM cocaine (see Sections 3.3.4.1 and 3.3.4.3) also stimulates pregnenolone synthesis, albeit with lower efficacy. Pregnenolone in turn acts as a NAM, thus dampening $\mathrm{CB}_{1} \mathrm{R}$-dependent effects in the brain, such as $\Delta^{9}$-THC-induced food intake, memory impairment, decrease in synaptic transmission, and increased accumbal dopamine release (see Sections 3.3.4.1 and 3.3.5.5) [406]. This well-positions pregnenolone in the list of potential $\mathrm{CB}_{1} \mathrm{R}$-inhibitor medicines without deleterious side effects. Other $\mathrm{CB}_{1} \mathrm{R}$-targeting strategies that involve glucocorticoid-induced illnesses are metabolic syndrome [977] and osteoporosis [652] among others.

\subsubsection{Other Atypical Targets}

The previously detailed off-target actions at opioid receptors are not exceptional: phytocannabinoids, endocannabinoids and other synthetic cannabinoid ligands are notorious for producing diverse off-target effects in the micromolar range [381,390,391,509,719,978-983] with unclear (patho)physiological significance. These must be taken into consideration, and whenever possible, cannabinoids should be used at the concentration not greater than $1 \mu \mathrm{M}$ in vitro. Nevertheless, studies directed to understand the molecular and physicochemical properties of off-target cannabinoid effects can help to design much more potent and selective ligands of testable therapeutic potential.

Several studies have documented therapeutic potential for phytocannabinoids in models where cannabinoid receptors were chemically or genetically ablated. Many times a compound's useful in vitro concentration range is too high, which anticipates that the therapeutic and the toxic in vivo doses would be too close to each other. For instance, (1) $\Delta^{9}$-THC competitively inhibits acetylcholinesterase by binding to its peripheral anionic binding site with the $K_{\mathrm{i}}$ of $10.2 \mu \mathrm{M}$, thereby preventing the enzyme from serving as a chaperon in the formation of $\beta$-amyloid fibrils [984]. (2) Micromolar $\Delta^{9}$-THC and other cannabinoids were found to inhibit monoamine oxidases $\mathrm{A}$ and $\mathrm{B}$, which may contribute to the antidepressant effects of cannabis [985]. (3) Micromolar concentrations of CBD and CBN, as well as WIN55212-2 (and its cannabinoid receptor inactive enantiomer, WIN55212-3) were found to inhibit a $\mathrm{Ca}^{2+}$ ionophore-induced interleukin-2 production in mouse splenocytes, even in the presence of the $C_{2} R$ antagonist/inverse agonist SR144528 [441] and the $C_{1} R$ antagonist/inverse agonist/GPR55 and GPR18 ligand SR141716A, and actually both SR141716A and SR144528 acted also as agonists [986]. (4) Micromolar concentrations of the non-psychoactive phytocannabinoids, CBC, CBD, CBDA, and CBG showed antitumoral and anticancer effects both in vivo and in vitro [522]. At those concentrations, phytocannabinoids were shown to interact with multiple targets of the endocannabinoid system, and their beneficial effects were not dependent on solely one protein [522].

\subsubsection{The Anti-inflammatory Effects of Ajulemic Acid}

More than 2 decades ago, the quest for $\Delta^{9}$-THC-like medicines which retain therapeutic usefulness without psychotropic activity has led to the discovery of a synthetic THC-COOH derivative called ajulemic acid (AJA) [987]. AJA has prolonged anti-inflammatory and analgesic action, which was virtually unrelated to $\mathrm{CB}_{1}$ or $\mathrm{CB}_{2}$ receptors. AJA in the low micromolar range can activate the peroxisome proliferator-activated receptor $\gamma$ (PPAR $\gamma$ ), being selective over the PPAR $\alpha$ and PPAR $\delta$ [988]. The PPAR $\gamma$ is a significant member of the nuclear receptor superfamily [989,990]. Intriguingly, 2-AG, noladin ether and NAGly (if not their COX-2 metabolites) are also activators of PPAR $\gamma$ [990,991]. Based on the potent actions of AJA, it came as no surprise that $\Delta^{9}$-THC and CBD also proved to be PPAR $\gamma$ activators: CBD and $\Delta^{9}$-THC induced slow vasorelaxation in rat aortae and 3T3-L1 fibroblast differentiation into adipocytes in a PPAR $\gamma$ antagonist- (GW9662) sensitive manner, which could be mimicked with the PPAR $\gamma$ agonist, rosiglitazone [992,993]. $\Delta^{9}$-THC and rosiglitazone (but surprisingly, not CBD or nabilone) protected a human neuroblastoma cell line via PPAR $\gamma$ activation from Parkinson's disease-relevant toxins (1-methyl-4phenylpyridinium - $\mathrm{MPP}^{+}$, paraquat and lactacystin) [994]. In the first clinical trial on patients with chronic neuropathic pain with or without allodynia and hyperalgesia, daily doses of 40 and $80 \mathrm{mg}$ AJA (also known as CT-3) proved more efficacious than placebo to reduce pain, without major adverse effects including psychoactivity [995,996]. The unexpected twists were those reports that found AJA to be more potent at the $C_{1} R$ and the $C_{2} R$ than at the PPAR $\gamma$, and to produce some psychoactivity at the useful analgesic dose [380,997-999]. It turns out that the contrasting findings were likely due to the impurity of the earlier AJA preparations, and that AJA is in fact a potent and selective $\mathrm{CB}_{2} \mathrm{R}$ agonist in the low nanomolar range [1000]. In fact, the highly purified AJA preparation is now termed as JBT101 which has 12-fold $C_{2}$ R-selectivity over the $C_{1} R$, while the original preparation is termed as HU239 which has 5-fold $\mathrm{CB}_{1}$ R-selectivity over the $\mathrm{CB}_{2} \mathrm{R}$ [1000]. JBT101 is a safe medicine with minor metabolism in the body [1001] and under the name Resunab ${ }^{\mathrm{TM}}$ (Corbus Pharmaceuticals) is currently being evaluated against inflammation and fibrosis in three phase II clinical trials. 


\section{CONCLUSIONS}

Our aim was to provide the reader with up-to-date information about the botany, psychobiology and therapeutic potential of cannabis. We advocate that the decision on cannabis legalization should not be the privilege of politicians, because researchers, health care professionals and social workers ought also to be involved in the discussion. It is not appropriate to promote the legalization of recreational cannabis based on its therapeutic potential. We presented numerous lines of evidence for the medical potential of cannabis constituents in a wide variety of diseases. Many clinical trials are already underway to market CBD and other inoffensive constituents to alleviate symptoms and mitigate the outcome of illnesses. This proves the concept that the cannabis plant is a true "treasure trove" [1002]. Yet, we should make it clear that whenever it seems meaningful to target the endocannabinoid system in a particular disease, it does not necessarily mean that cannabis would also serve as a remedy. On the contrary: first, because several health conditions require the inhibition rather than the activation of cannabinoid receptors, and second, cannabis is a double-edged sword and can do just as much harm as good. In our apposite example, cannabis is somewhat analogous to the way the mafia works: once you get involved you have hard time to turn your back on it. It will protect your cells in hour of need, but it will also take its protection racket from the healthy cells. Unfortunately, it does not always depend on the discretion and the good sense of cannabis users whether they open the treasure trove or instead, Pandora's box.

\section{CONFLICT OF INTEREST}

The authors declare no conflict of interest.

\section{ACKNOWLEDGEMENTS}

We are grateful to Dr. Samira G. Ferreira, Center for Neuroscience and Cell Biology of Coimbra, University of Coimbra, Portugal, for the immunohistochemistry and some of the neurochemistry experiments shown in Figs. 9 and $\mathbf{1 0 .}$ We would like to thank Dr. Samira G. Ferreira and Judit Solymosi for their precious help with the list of References. We are also thankful to Dr. Catherine Ledent and Ms. Catherine Vroonen, IRIBHM, Université Libre de Bruxelles, Brussels B-1070 for the kind supply of the $\mathrm{CB}_{1} \mathrm{R}-/-$ mice and their wild-type littermates. We are grateful to Dr. Tibor Harkany, Department of Molecular Neurosciences, Center for Brain Research, Medical University of Vienna, Vienna, Austria, for his kind contribution to Fig. 7. We also extend our gratitude to Ernest Small (Agriculture and Agri-Food Canada, Ottawa) for providing a SEM picture for Fig. 3 and would like to thank Béla Dános, Department of Plant Anatomy, Eötvös Loránd University, Budapest, Hungary, for discussions about the botanical part of the review. This work was supported by the Janos Bolyai Research Scholarship of the Hungarian Academy of Sciences (to K.S.), and by FEDER Funds through the Competitiveness Factors Operational Program - COMPETE 2020 and by National Funds through FCT - Foundation for Science and
Technology, in the scope of the Strategic Project: POCI-010145-FEDER-007440, and by national funds PTDC/SAUOSM/105663/2008 and PTDC/SAU-NEU/100729/2008 from FCT (to A.K.). 


\section{ABBREVIATIONS}

-/-, KO = "knockout" mouse lacking a specific protein (receptor)

2-AG = sn-2-arachidonoylglycerol

2-AGPI = 2-arachidonoyl-sn-glycero-3-phosphoinositol

$5-\mathrm{HT}_{1 \mathrm{~A} / 2 \mathrm{~A} / 3} \mathrm{R}=$ serotonin $5-\mathrm{HT}_{1 \mathrm{~A} / 2 \mathrm{~A} / 3}$ receptor

$\alpha_{2} \mathrm{R}=\alpha_{2}$ adrenoceptor

$\mathrm{A}_{1 / 2 \mathrm{~A}} \mathrm{R}(\mathrm{s})=$ adenosine $\mathrm{A}_{1 / 2 \mathrm{~A}}$ receptor(s)

$\mathrm{ABDH} 4 / 6 / 12=\alpha, \beta$ domain serine hydrolase 4 or 6 or 12

abn-CBD = abnormal cannabidiol

ACEA = arachidonoyl-2'-chloroethylamide

ACh $=$ acetylcholine

ACPA = arachidonoylcyclopropylamide

ADTR = agonist-directed trafficking of response

AJA = ajulemic acid (CT-3)

Akt $=$ protein kinase $\mathrm{B}$

Bax $=$ Bcl-2-associated $\mathrm{X}$ protein

$\mathrm{Bcl}=\mathrm{B}$-cell lymphoma oncogene

BrdU = bromodeoxyuridine

$\left[\mathrm{Ca}^{2+}\right]_{\mathrm{i}}=$ intracellular $\mathrm{Ca}^{2+}$ concentration

$\mathrm{Ca}^{2+}$-ER/RER $=\mathrm{Ca}^{2+} /$ receptor dependent endocannabinoid release

CaMKII $=\mathrm{Ca}^{2+} /$ calmodulin-dependent protein kinase II

cAMP = 3',5'-cyclic adenosine monophosphate

$\mathrm{CB}_{1} \mathrm{R}(\mathrm{s})$ and $\mathrm{CB}_{2} \mathrm{R}(\mathrm{s})=$ cannabinoid $\mathrm{CB}_{1}$ and $\mathrm{CB}_{2}$ receptor(s)

$\mathrm{CBC}=$ cannabichromene

CBCA = cannabichromenic acid

$\mathrm{CBCV}=$ cannabichromevarin

CBD $=(-)$-cannabidiol

CBDA = cannabidiolic acid

$\mathrm{CBDV}=$ cannabidivarin

$\mathrm{CBG}=$ cannabigerol

CBGA = cannabigerolic acid

$\mathrm{CBGV}=$ cannabigerovarin

$\mathrm{CBN}=$ cannabinol
cGMP = cyclic guanosine monophosphate

$\mathrm{CHO}=$ Chinese hamster ovary

COX-2 = cyclooxygenase -2

$\mathrm{CRIP} 1 \mathrm{a} / \mathrm{b}=\mathrm{CB}_{1} \mathrm{R}$ interacting protein $1 \mathrm{a}$ or $1 \mathrm{~b}$

CUD = cannabis use disorder

$\delta \mathrm{OpR}=\delta$ opioid receptor

$\Delta^{8}$-THC $=\Delta^{8}$-tetrahydrocannabinol

$\Delta^{9}$-THC $=\Delta^{9}$-tetrahydrocannabinol

$\Delta^{9}$-THCA $=\Delta^{9}-$ THC acid

$\Delta^{9}$-THCV $=\Delta^{9}$-tetrahydrocannabivarin

$\mathrm{DA}=$ divarinic acid

$\mathrm{D}_{1 / 2} \mathrm{R}(\mathrm{s})=$ dopamine $\mathrm{D}_{1 / 2}$ receptor(s)

DAG $=$ sn-2-diacylglycerol

DAGL $\alpha / \beta=$ sn-2-DAG lipase $\alpha$ or $\beta$

$\mathrm{DAT}=$ dopamine transporter

DEA = docosatetraenylethanolamide

DLE $=$ delusional-like experiences

DSI/DSE = depolarization-induced suppression of inhibition/excitation

EGFR = epidermal growth factor receptor

EMT $=$ putative endocannabinoid membrane transporter

ERK1/2 = extracellular regulated kinase $1 / 2$

FAAH-1/2 = fatty acid aminohydrolase- 1 or 2

FGFR $=$ fibroblast growth factor receptor

FLAT $=$ FAAH-like anandamide transporter

$\mathrm{GABA}=\gamma$-aminobutyric acid

GASP1 = GPCR-associated sorting protein 1

GIRKs = inwardly rectifying $\mathrm{K}^{+}$channels

GOT = geranylpyrophosphate:olivetolate geranyltransferase

GPCR(s) $=\mathrm{G}$ protein-coupled receptor(s)

GPP = geranyl pyrophosphate/geranyl diphosphate

GPR18 = GPR55, G protein-coupled receptor 18 or 55

GRKs $=$ GPCR kinases

$\left[{ }^{35} \mathrm{~S}\right]$-GTP $\gamma \mathrm{S}=\left[{ }^{35} \mathrm{~S}\right]$-guanosine 5'-O-[gammathio]triphosphate

HEA = homo- $\gamma$-linolenylethanolamide

HCN = cyclic nucleotide-gated $(\mathrm{HCN})$ channels 
HEK293 = human embryonic kidney cell line 293

JNKs = c-Jun N-terminal kinases

$\kappa \mathrm{OpR}=\kappa$ opioid receptor

KynA = kynurenic acid

LPI = L- $\alpha$-lysophosphatidylinositol

LTP, LTD = long-term potentiation, long-term depression

LysoPtdGlc = lysophosphatidyl- $\beta$-D-glucoside

$\mu \mathrm{OpR}=\mu$ opioid receptor

$\mathrm{M}_{1 / 3} \mathrm{R}(\mathrm{s})=$ muscarinic acetylcholine $\mathrm{M}_{1}$ or $\mathrm{M}_{3}$ receptor(s)

MAGL = monoacylglycerol lipase

MAPKs $=$ mitogen-activated protein kinases

$\operatorname{mGluR}_{5}(\mathrm{~s})=$ metabotropic glutamate receptor(s) 5

$\mathrm{MPP}^{+}=1$-methyl-4-phenylpyridinium

MSI/MSE = metabotropic stimulation-induced suppression of inhibition/excitation

$\operatorname{MSN}(\mathrm{s})=$ medium spiny neuron(s)

$\mathrm{nAChR}(\mathrm{s})=$ nicotinic acetylcholine receptor(s)

NADA $=N$-arachidonoyl dopamine

NAGly $=N$-arachidonoylglycine

NAM $=$ negative allosteric modulator

NAPE $=N$-arachidonoyl-phosphatidylethanolamine

NFAT $=$ nuclear factor of activated T-cells

NMR = nuclear magnetic resonance spectroscopy

$\mathrm{OA}=$ olivetolic acid

$\mathrm{OAC}=\mathrm{OA}$ cyclase

OLDA $=N$-oleoyldopamine

p38 $($ MAPK $)=$ p38 mitogen-activated protein kinases

PAM = positive allosteric modulator

$\mathrm{PD}=$ Parkinson's disease

$\mathrm{PI}_{3} \mathrm{~K}=$ phosphoinositide 3-kinase

$\mathrm{PIP}_{2}=$ phosphatidylinositol 4,5-bisphosphate

$\mathrm{PKA}=$ protein kinase $\mathrm{A}$

PLC $\beta=$ phospholipase $C \beta$

PPAR $\alpha / \delta / \gamma=$ peroxisome proliferator-activated receptor $\alpha$ or $\delta$ or $\gamma$

PTSD $=$ post-traumatic stress disorder

RhoA = Ras homolog gene family member $\mathrm{A}$
RIM1/2 $\alpha=$ Rab3-interacting molecule- $1 \alpha$ or $-2 \alpha$

ROCK $=$ Rho-associated protein kinase

ROS $=$ reactive oxygen species

$\mathrm{RTK}(\mathrm{s})$ = receptor tyrosine kinase(s)

Src = proto-oncogene tyrosine-protein kinase Src

STAT3 $=$ signal transducer and activator of transcription 3

STDP = spike-timing-dependent plasticity

TKS = tetraketide synthase

TrkB = tropomyosin receptor kinase B (brain-derived neurotrophic factor receptor)

$\mathrm{TRP}=$ transient receptor potential

$\mathrm{TRPA}_{1} \mathrm{R}=\mathrm{TRP}$ ankyrin family type 1 receptor

$\mathrm{TRPC}_{1} \mathrm{R}=\mathrm{TRP}$ canonical family type 1 receptor

$\mathrm{TRPM}_{8}=\mathrm{TRP}$ melastatin (melanoma suppressor) family type 8 receptor

$\mathrm{TRPV}_{1 / 2 / 4} \mathrm{R}=\mathrm{TRP}$ vanilloid family type 1 or 2 or 4 ion channels

VGCCs $=$ voltage-gated $\mathrm{Ca}^{2+}$ channels

$\mathrm{VTA}=$ ventral tegmental area

WT = wild-type

\section{REFERENCES}

[1] Petrovic, M.; Debeljak, Z.; Kezic, N.; Dzidara, P. Relationship between cannabinoids content and composition of fatty acids in hempseed oils. Food Chem., 2015, 170, 218-225.

[2] Small, E. Evolution and classification of Cannabis sativa (marijuana, hemp) in relation to human utilization. Bot. Rev., 2015, 81(3), 189294.

[3] Merlin, M.D. Archaeological evidence for the tradition of psychoactive plant use in the old world. Econ. Bot., 2003, 57(3), 295323.

[4] Bayer, I. A drogok történelme (History of drugs); Aranyhal kiadó: Budapest, 2000.

[5] Beutler, J.A.; der Marderosian, A.H. Chemotaxonomy of Cannabis I. Crossbreeding between Cannabis sativa and C. ruderalis, with analysis of cannabinoid content. Econ. Bot., 1978, 32(4), 387-397.

[6] Hillig, K.W.; Mahlberg, P.G. A chemotaxonomic analysis of cannabinoid variation in Cannabis (Cannabaceae). Am. J. Bot., 2004, 91(6), 966-975.

[7] Hillig, K. Genetic evidence for speciation in Cannabis (Cannabaceae). Genet. Resour. Crop Evol., 2005, 52(2), 161-180.

[8] Russo, E.B. History of Cannabis and its preparations in saga, science, and sobriquet. Chem. Biodivers., 2007, 4(8), 1614-1648.

[9] Small, E. Interfertility and chromosomal uniformity in Cannabis. Can. J. Bot., 1972, 50(9), 1947-1949.

[10] Kojoma, M.; Seki, H.; Yoshida, S.; Muranaka, T. DNA polymorphisms in the tetrahydrocannabinolic acid (THCA) synthase gene in "drug-type” and "fiber-type" Cannabis sativa L. Forensic Sci. Int., 2006, 159, 132-140.

[11] Marks, M.D.; Tian, L.; Wenger, J.P.; Omburo, S.N.; Soto-Fuentes, W.; He, J.; Gang, D.R.; Weiblen, G.D.; Dixon, R.A. Identification of candidate genes affecting $\Delta 9$-tetrahydrocannabinol biosynthesis in Cannabis sativa. J. Exp. Bot., 2009, 60(13), 3715-3726.

[12] van Bakel, H.; Stout, J.M.; Cote, A.G.; Tallon, C.M.; Sharpe, A.G.; Hughes, T.R.; Page, J.E. The draft genome and transcriptome of Cannabis sativa. Genome Biol., 2011, 12(10), R102. 
[13] Gilmore, S.; Peakall, R.; Robertson, J. Organelle DNA haplotypes reflect crop-use characteristics and geographic origins of Cannabis sativa. Forensic Sci. Int., 2007, 172(2-3), 179-190.

[14] Weiblen, G.D.; Wenger, J.P.; Craft, K.J.; ElSohly, M.A.; Mehmedic, Z.; Treiber, E.L.; Marks, M.D. Gene duplication and divergence affecting drug content in Cannabis sativa. New Phytol., 2015, 208(4), 1241-1250.

[15] Pate, D.W. Chemical ecology of Cannabis. J. Int. Hemp Assoc., 1994, 2(29), 32-37.

[16] United Nations Office on Drugs and Crime. Recommended methods for the identification and analysis of cannabis and cannabis products; United Nations Publication, 2009.

[17] Small, E.; Naraine, S.G.U. Size matters: evolution of large drugsecreting resin glands in elite pharmaceutical strains of Cannabis sativa (marijuana). Genet. Resour. Crop Evol., 2015, 63(2), 349-359.

[18] Ecsedy, I.; Bökönyi, S. The people of the pit-grave kurgans in eastern Hungary; Akadémiai Kiadó: Budapest, 1979.

[19] Ben Amar, M. Cannabinoids in medicine: A review of their therapeutic potential. J. Ethnopharmacol., 2006, 105(1-2), 1-25.

[20] Hazekamp, A.; Grotenhermen, F. Review on clinical studies with cannabis and cannabinoids 2005-2009. Cannabinoids, 2010, 5(SI), 121.

[21] Owens, B. Drug development: The treasure chest. Nature, 2015, 525(7570), S6-8.

[22] Shelef, A.; Barak, Y.; Berger, U.; Paleacu, D.; Tadger, S.; Plopsky, I.; Baruch, Y. Safety and efficacy of medical cannabis oil for behavioral and psychological symptoms of dementia: An-open label, add-on, pilot study. J. Alzheimers Dis., 2016, 51(1), 15-19.

[23] Tzadok, M.; Uliel-Siboni, S.; Linder, I.; Kramer, U.; Epstein, O.; Menascu, S.; Nissenkorn, A.; Yosef, O.B.; Hyman, E.; Granot, D.; Dor, M.; Lerman-Sagie, T.; Ben-Zeev, B. CBD-enriched medical cannabis for intractable pediatric epilepsy: The current Israeli experience. Seizure, 2016, 35, 41-44.

[24] Clark, P.A.; Capuzzi, K.; Fick, C. Medical marijuana: medical necessity versus political agenda. Med. Sci. Monit., 2011, 17(12), RA249-RA261.

[25] Leung, L. Cannabis and its derivatives: review of medical use. J. Am. Board Fam. Med., 2011, 24(4), 452-462.

[26] Gould, J. The cannabis crop. Nature, 2015, 525(7570), S2-S3.

[27] United Nations Office on Drugs and Crime. World Drug Report 2014. http://www.unodc.org/wdr/en/cannabis.html. (Accessed September 10, 2015).

[28] Mehmedic, Z.; Chandra, S.; Slade, D.; Denham, H.; Foster, S.; Patel, A.S.; Ross, S.A.; Khan, I.A.; ElSohly, M.A. Potency trends of $\Delta 9$ THC and other cannabinoids in confiscated cannabis preparations from 1993 to 2008. J. Forensic Sci., 2010, 55(5), 1209-1217.

[29] ElSohly, M.A.; Gul, W. In: Handbook of Cannabis; Pertwee, R.G., Ed. Oxford University Press: New York, 2014; pp. 3-22.

[30] Brenneisen, R. In: Marijuana and the Cannabinoids SE - 2; ElSohly, M.A., Ed. Forensic Science And Medicine; Humana Press: New Jersey, 2007; pp. 17-49.

[31] Docimo, T.; Consonni, R.; Coraggio, I.; Mattana, M. Early phenylpropanoid biosynthetic steps in Cannabis sativa: Link between genes and metabolites. Int. J. Mol. Sci., 2013, 14, 13626-13644.

[32] ElSohly, M.A.; Slade, D. Chemical constituents of marijuana: The complex mixture of natural cannabinoids. Life Sci., 2005, 78, 539548.

[33] Ibrahim, A.K.; Radwan, M.M.; Ahmed, S.A.; Slade, D.; Ross, S.A.; Elsohly, M.A.; Khan, I.A. Microbial metabolism of cannflavin A and B isolated from Cannabis sativa. Phytochemistry, 2010, 71(8-9), 1014-1019.

[34] Martin, L.; Morison Smith, D.; Farmilo, C.G. Essential oil from fresh Cannabis sativa and its use in identification. Nature, 1961, 191(4790), 774-776.

[35] Nigam, M.C.; Handa, K.L.; Nigam, I.C.; Levi, L. Essential oils and their constituents XXIX. The essential oil of marihuana: Composition of genuine Indian Cannabis sativa L. Can. J. Chem., 1965, 43(12), 3372-3376.

[36] Turner, C.E.; ElSohly, M.A.; Boeren, E.G. Constituents of Cannabis sativa L. XVII. A review of natural constituents. J. Nat. Prod., 1980, 43, 169-234.

[37] Russo, E.B. Taming THC: potential cannabis synergy and phytocannabinoid-terpenoid entourage effects. Br. J .Pharmacol., 2011, 163(7), 1344-1364.

[38] Flemming, T.; Muntendam, R.; Steup, C.; Kayser, O. In: Bioactive Heterocycles IV. Topics in Heterocyclic Chemistry Vol. 10; Khan, M.T.H., Ed. Springer: Berlin, 2007; pp. 1-42.

[39] Rätsch, C. Marijuana medicine: A world tour of the healing and visionary powers of Cannabis; Healing Arts Press: Rochester, 2001.

[40] Stahl, E.; Kunde, R. Die Leitsubstanzen der Haschisch-Suchhunde. Kriminalistik, 1973, 9, 385-388.

[41] Ross, S.A.; ElSohly, M.A. The volatile oil composition of fresh and air-dried buds of Cannabis sativa. J. Nat. Prod., 1996, 59(1), 49-51.

[42] Fischedick, J.T.; Hazekamp, A.; Erkelens, T.; Choi, Y.H.; Verpoorte, R. Metabolic fingerprinting of Cannabis sativa L., cannabinoids and terpenoids for chemotaxonomic and drug standardization purposes. Phytochemistry, 2010, 71(17-18), 2058-2073.

[43] Mansouri, H.; Asrar, Z. Effects of abscisic acid on content and biosynthesis of terpenoids in Cannabis sativa at vegetative stage. Biol. Plant., 2012, 56(1), 153-156.

[44] Mansouri, H.; Asrar, Z.; Amarowicz, R. The response of terpenoids to exogenous gibberellic acid in Cannabis sativa $\mathrm{L}$. at vegetative stage. Acta Physiol. Plant., 2011, 33, 1085-1091.

[45] Mansouri, H.; Asrar, Z.; Szopa, J. Effects of ABA on primary terpenoids and $\Delta 9$-tetrahydrocannabinol in Cannabis sativa L. at flowering stage. Plant Growth Regul., 2009, 58, 269-277.

[46] Mansouri, H.; Salari, F.; Asrar, Z. Ethephon application stimulats cannabinoids and plastidic terpenoids production in Cannabis sativa at flowering stage. Ind. Crops Prod., 2013, 46, 269-273.

[47] Günnewich, N.; Page, J.E.; Köllner, T.G.; Degenhardt, J.; Kutchan, T.M. Functional expression and characterization of trichome-specific (-)- limonene synthase and $(+)-\alpha$-pinene synthase from Cannabis sativa. Nat. Prod. Commun., 2007, 2, 223-232.

[48] Toyota, M.; Shimamura, T.; Ishii, H.; Renner, M.; Braggins, J.; Asakawa, Y. New bibenzyl cannabinoid from the New Zealand liverwort Radula marginata. Chem. Pharm. Bull. (Tokyo), 2002, 50(10), 1390-1392.

[49] Toyota, M.; Kinugawa, T.; Asakawa, Y. Bibenzyl cannabinoid and bisbibenzyl derivative from the liverwort Radula perrottetii. Phytochemistry, 1994, 37(3), 859-862.

[50] Bohlmann, F.; Hoffmann, E. Cannabigerol-ähnliche verbindungen aus Helichrysum umbraculigerum. Phytochemistry, 1979, 18(8), 1371-1374.

[51] Thakur, G.A.; Duclos, R.I.; Makriyannis, A. Natural cannabinoids: Templates for drug discovery. Life Sci., 2005, 78, 454-466.

[52] Kimura, M.; Okamoto, K. Distribution of tetrahydrocannabinolic acid in fresh wild cannabis. Experientia, 1970, 26(8), 819-820.

[53] Yamauchi, T.; Shoyama, Y.; Aramaki, H.; Azuma, T.; Nishioka, I. Tetrahydrocannabinolic acid, a genuine substance of tetrahydrocannabinol. Chem. Pharm. Bull., 1967, 15, 1075-1076.

[54] Shoyama, Y.; Yagi, M.; Nishioka, I.; Yamauchi, T. Biosynthesis of cannabinoid acids. Phytochemistry, 1975, 14(10), 2189-2192.

[55] Taura, F.; Sirikantaramas, S.; Shoyama, Y.; Shoyama, Y.; Morimoto, S. Phytocannabinoids in Cannabis sativa: Recent studies on biosynthetic enzymes. Chem. Biodivers., 2007, 4(8), 1649-1663.

[56] Wollner, H.J.; Matchett, J.R.; Levine, J.; Loewe, S. Isolation of a physiologically active tetrahydrocannabinol from Cannabis sativa resin. J. Am. Chem. Soc., 1942, 64(1), 26-29.

[57] Gaoni, Y.; Mechoulam, R. Isolation, structure and partial synthesis of an active constituent of hashish. J. Am. Chem. Soc., 1964, 86(8), 1646-1647.

[58] Ashton, C.H.; Moore, P.B. Endocannabinoid system dysfunction in mood and related disorders. Acta Psychiatr. Scand., 2011, 124(4), 250-261.

[59] Carlini, E.A.; Cunha, J.M. Hypnotic and antiepileptic effects of cannabidiol. J. Clin. Pharmacol., 1981, 21(S1), 417S-427S.

[60] Mechoulam, R.; Parker, L.A.; Gallily, R. Cannabidiol: An overview of some pharmacological aspects. J. Clin. Pharmacol., 2002, 42(S1), 11S-19S.

[61] Mechoulam, R.; Peters, M.; Murillo-Rodriguez, E.; Hanuš, L.O. Cannabidiol - Recent advances. Chem. Biodivers., 2007, 4(8), 16781692.

[62] Oláh, A.; Tóth, B.I.; Borbíró, I.; Sugawara, K.; Szöllősi, A.G.; Czifra, G.; Pál, B.; Ambrus, L.; Kloepper, J.; Camera, E.; Ludovici, M.; Picardo, M.; Voets, T.; Zouboulis, C.C.; Paus, R.; Bíró, T. Cannabidiol exerts sebostatic and antiinflammatory effects on human sebocytes. J. Clin. Invest., 2014, 124(9), 3713-3724.

[63] DeLong, G.T.; Wolf, C.E.; Poklis, A.; Lichtman, A.H. Pharmacological evaluation of the natural constituent of Cannabis sativa, cannabichromene and its modulation by $\Delta 9$ tetrahydrocannabinol. Drug Alcohol Depend., 2010, 112(1-2), 126133.

[64] Radwan, M.M.; ElSohly, M.A.; Slade, D.; Ahmed, S.A.; Khan, I.A.; Ross, S.A. Biologically active cannabinoids from high-potency Cannabis sativa. J. Nat. Prod., 2009, 72(5), 906-911.

[65] Russo, E.; Guy, G.W. A tale of two cannabinoids: The therapeutic 
rationale for combining tetrahydrocannabinol and cannabidiol. Med. Hypotheses, 2015, 66(2), 234-246.

[66] Ferrè, L.; Nuara, A.; Pavan, G.; Radaelli, M.; Moiola, L.; Rodegher, M.; Colombo, B.; Keller Sarmiento, I.; Martinelli, V.; Leocani, L.; Martinelli Boneschi, F.; Comi, G.; Esposito, F. Efficacy and safety of nabiximols (Sativex ${ }^{\circledR}$ ) on multiple sclerosis spasticity in a real-life Italian monocentric study. Neurol. Sci., 2015, 1-8.

[67]

http://www.gwpharm.com/GW\%20Pharmaceuticals\%20and\%20Otsu ka\%20Announce\%20Results\%20in\%20First\%20of\%20Three\%20 Sativex\%20Phase\%203\%20Cancer\%20Pain\%20Trials.aspx (Accessed 26 June, 2016)

[68] http://www.gwpharm.com/GWPOtsukaResults271015.aspx (Accessed 26 June, 2016)

[69] de Meijer, E.P.M.; Hammond, K.M.; Micheler, M. The inheritance of chemical phenotype in Cannabis sativa L. (III): Variation in cannabichromene proportion. Euphytica, 2009, 165, 293-311.

[70] Pacifico, D.; Miselli, F.; Carboni, A.; Moschella, A.; Mandolino, G. Time course of cannabinoid accumulation and chemotype development during the growth of Cannabis sativa L. Euphytica, 2008, 160, 231-240.

[71] Fetterman, P.S.; Keith, E.S.; Waller, C.W.; Guerrero, O.; Doorenbos, N.J.; Quimby, M.W. Mississippi-grown cannabis sativa L.: Preliminary observation on chemical definition of phenotype and variations in tetrahydrocannabinol content versus age, sex, and plant part. J. Pharm. Sci., 1971, 60(8), 1246-1249.

[72] Barni-Comparini, I.; Ferri, S.; Centini, F. Cannabinoid level in the leaves as a tool for the early discrimination of Cannabis chemiovariants. Forensic Sci. Int., 1984, 24(1), 37-42.

[73] Mandolino, G.; Bagatta, M.; Carboni, A.; Ranalli, P.; de Meijer, E.P.M. Qualitative and quantitative aspects of the inheritance of chemical phenotype in Cannabis. J. Ind. Hemp, 2003, 8(2), 51-72.

[74] Bócsa, I.; Máthé, P.; Hangyel, L. Effect of nitrogen on tetrahydrocannabinol (THC) content in hemp (Cannabis sativa L.) leaves at different positions. J. Int. Hemp Assoc., 1997, 4(2), 78-79.

[75] Vogelmann, A.F.; Turner, J.C.; Mahlberg, P.G. Cannabinoid occurrence in seedlings of Cannabis sativa L.: Quantitation in seedlings of known age and primary leaf length. Bot. Gaz., 1987, 148(4), 468-474.

[76] Mechtler, K.; Bailer, J.; de Hueber, K. Variations of $\Delta 9$-THC content in single plants of hemp varieties. Ind. Crops Prod., 2004, 19(1), 1924.

[77] de Meijer, E.P.M.; Bagatta, M.; Carboni, A.; Crucitti, P.; Moliterni, V.; Ranalli, P.; Mandolino, G. The inheritance of chemical phenotype in Cannabis sativa L. Genetics, 2003, 163, 335-346.

[78] Small, E.; Beckstead, H. Common cannabinoid phenotypes in 350 stocks of Cannabis. Lloydia, 1973, 36(2), 144-165.

[79] Small, E.; Beckstead, H.D. Cannabinoid phenotypes in Cannabis sativa. Nature, 1973, 245(5421), 147-148.

[80] de Meijer, E.P.M.; Hammond, K.M. The inheritance of chemical phenotype in Cannabis sativa L. (II): Cannabigerol predominant plants. Euphytica, 2005, 145, 189-198.

[81] de Meijer, E.P.M.; Hammond, K.M.; Sutton, A. The inheritance of chemical phenotype in Cannabis sativa L. (IV): Cannabinoid-free plants. Euphytica, 2009, 168, 95-112.

[82] Sutipatanasomboon, A.; Panvisavas, N. Discrimination of "fibertype" and "drug-type" Cannabis sativa L. by fluorescent duplex PCR. Forensic Sci. Int. Genet. Suppl. Ser., 2011, 3(1), e522-e523.

[83] Rotherham, D.; Harbison, S.A. Differentiation of drug and non-drug Cannabis using a single nucleotide polymorphism (SNP) assay. Forensic Sci. Int., 2011, 207(1-3), 193-197.

[84] Hewavitharana, A.K.; Golding, G.; Tempany, G.; King, G.; Holling, N. Quantitative GC-MS analysis of D(9)-tetrahydrocannabinol in fiber hemp varieties. J. Anal. Toxicol., 2005, 29(4), 258-261.

[85] Commission Regulation (EC) No. 1124/2008 of 12 November 2008. Off. J. Eur. Union, 2008, L303, 7-9.

[86] Small, E.; Jui, P.Y.; Lefkovitch, L.P. A numerical taxonomic analysis of Cannabis with special reference to species delimitation. Syst. Bot., 1976, 1(1), 67-84.

[87] Appendino, G.; Gibbons, S.; Giana, A.; Pagani, A.; Grassi, G.; Stavri, M.; Smith, E.; Rahman, M.M. Antibacterial cannabinoids from Cannabis sativa: A structure-activity study. J. Nat. Prod., 2008, 71(8), 1427-1430.

[88] McPartland, J.; Clarke, R.; Watson, D. Hemp diseases and pests: management and biological control - an advanced treatise; CABI: Wallingford, 2000.

[89] Morimoto, S.; Tanaka, Y.; Sasaki, K.; Tanaka, H.; Fukamizu, T.; Shoyama, Y.; Shoyama, Y.; Taura, F. Identification and characterization of cannabinoids that induce cell death through mitochondrial permeability transition in cannabis leaf cells. J. Biol. Chem., 2007, 282, 20739-20751.

[90] Pate, D.W. Possible role of ultraviolet radiation in evolution of Cannabis chemotypes. Econ. Bot., 1983, 37(4), 396-405.

[91] Lydon, J.; Teramura, A.H.; Coffman, C.B. UV-B radiation effects on photosynthesis, growth and cannabinoid production of two Cannabis sativa chemotypes. Photochem. Photobiol., 1987, 46(2), 201-206.

[92] Lydon, J.; Teramura, A.H. Photochemical decomposition of cannabidiol in its resin base. Phytochemistry, 1987, 26(4), 12161217.

[93] Raharjo, T.J.; Verpoorte, R. Methods for the analysis of cannabinoids in biological materials: a review. Phytochem. Anal., 2004, 15, 79-94.

[94] de Backer, B.; Debrus, B.; Lebrun, P.; Theunis, L.; Dubois, N. Decock, L.; Verstraete, A.; Hubert, P.; Charlier, C. Innovative development and validation of an HPLC/DAD method for the qualitative and quantitative determination of major cannabinoids in cannabis plant material. J. Chromatogr. B Anal. Technol. Biomed. Life Sci., 2009, 877(32), 4115-4124.

[95] Flores-Sanchez, I.J.; Verpoorte, R. PKS activities and biosynthesis of cannabinoids and flavonoids in Cannabis sativa L. plants. Plant Cell Physiol., 2008, 49(12), 1767-1782.

[96] Muntendam, R.; Happyana, N.; Erkelens, T.; Bruining, F.; Kayser, O. Time dependent metabolomics and transcriptional analysis of cannabinoid biosynthesis in Cannabis sativa var. Bedrobinol and Bediol grown under standardized condition and with genetic homogeneity. Online Int. J. Med. Plants Res., 2012, 1(2), 31-40.

[97] Fairbairn, J.W. The trichomes and glands of Cannabis sativa L. UN Bull. Narcotics, 1972, 24, 29-33.

[98] Lanyon, V.S.; Turner, J.C.; Mahlberg, P.G. Quantitative analysis of cannabinoids in the secretory product from capitate-stalked glands of Cannabis sativa L. (Cannabaceae). Bot. Gaz., 1981, 142(3), 316-319.

[99] Turner, J.C.; Hemphill, J.K.; Mahlberg, P.G. Gland distribution and cannabinoid content in clones of Cannabis sativa L. Am. J. Bot, 1977, 64(6), 687-693.

[100] Turner, J.C.; Hemphill, J.K.; Mahlberg, P.G. Quantitative determination of cannabinoids in individual glandular trichomes of Cannabis sativa L. (Cannabaceae). Am. J. Bot., 1978, 65(10), 11031106.

[101] Stolker, A.A.M.; van Schoonhoven, J.; de Vries, A.J.; BobeldijkPastorova, I.; Vaes, W.H.J.; van den Berg, R. Determination of cannabinoids in cannabis products using liquid chromatography-ion trap mass spectrometry. J. Chromatogr. A, 2004, 1058(1-2), 143-151.

[102] Oroszlán, P.; Verzár-Petri, G.; Mincsovics, E.; Székely, T. Separation, quantitation and isolation of cannabinoids from Cannabis sativa L. by overpressured layer chromatography. J. Chromatogr., 1987, 388, 217-224.

[103] Szabady, B.; Hidvégi, E.; Nyiredy, S. Determination of neutral cannabinoids in hemp samples by overpressured-layer chromatography. Chromatographia, 2002, 56, S165-S168.

[104] Cascini, F.; Passerotti, S.; Martello, S. A real-time PCR assay for the relative quantification of the tetrahydrocannabinolic acid (THCA) synthase gene in herbal Cannabis samples. Forensic Sci. Int., 2012, 217, 134-138.

[105] Galand, N.; Ernouf, D.; Montigny, F.; Dollet, J.; Pothier, J. Separation and identification of cannabis components by different planar chromatography techniques (TLC, AMD, OPLC). J. Chromatogr. Sci., 2004, 42(3), 130-134.

[106] Miller Coyle, H.; Palmbach, T.; Juliano, N.; Ladd, C.; Lee, H. C. An overview of DNA methods for the identification and individualization of marijuana. Croat. Med. J., 2003, 44, 315-321.

[107] Fellermeier, M.; Zenk, M.H. Prenylation of olivetolate by a hemp transferase yields cannabigerolic acid, the precursor of tetrahydrocannabinol. FEBS Lett., 1998, 427, 283-285.

[108] Sirikantaramas, S.; Taura, F.; Tanaka, Y.; Ishikawa, Y.; Morimoto, S.; Shoyama, Y. Tetrahydrocannabinolic acid synthase, the enzyme controlling marijuana psychoactivity, is secreted into the storage cavity of the glandular trichomes. Plant Cell Physiol., 2005, 46(9), 1578-1582.

[109] Taura, F.; Morimoto, S.; Shoyama, Y.; Mechoulam, R. First direct evidence for the mechanism of delta-1-tetrahydrocannabinolic acid biosynthesis. J. Am. Chem. Soc., 1995, 117(38), 9766-9767.

[110] Morimoto, S.; Komatsu, K.; Taura, F.; Shoyama, Y. Purification and characterization of cannabichromenic acid synthase from Cannabis sativa. Phytochemistry, 1998, 49, 1525-1529.

[111] Morimoto, S.; Komatsu, K.; Taura, F.; Shoyama, Y. Enzymological evidence for cannabichromenic acid biosynthesis. J. Nat. Prod., 1997, 60(8), 854-857. 
[112] Taura, F.; Morimoto, S.; Shoyama, Y. Purification and characterization of cannabidiolic-acid synthase from Cannabis sativa L . J. Biol. Chem., 1996, 271(29), 17411-17416.

[113] Taura, F.; Sirikantaramas, S.; Shoyama, Y.; Yoshikai, K.; Shoyama, Y.; Morimoto, S. Cannabidiolic-acid synthase, the chemotypedetermining enzyme in the fiber-type Cannabis sativa. FEBS Lett., 2007, 581, 2929-2934.

[114] Sirikantaramas, S.; Morimoto, S.; Shoyama, Y.; Ishikawa, Y.; Wada, Y.; Shoyama, Y.; Taura, F. The gene controlling marijuana psychoactivity. Molecular cloning and heterologous expression of $\Delta$ 1-tetrahydrocannabinolic acid synthase from Cannabis sativa L. J. Biol. Chem., 2004, 279(38), 39767-39774.

[115] de Zeeuw, R.A.; Wijsbek, J.; Breimer, D.D.; Vree, T.B.; van Ginneken, C.A.; van Rossum, J.M. Cannabinoids with a propyl side chain in Cannabis. Occurrence and chromatographic behaviour. Science, 1972, 175, 778-779.

[116] Shoyama, Y.; Hirano, H.; Nishioka, I. Biosynthesis of propyl cannabinoid acid and its biosynthetic relationship with pentyl and methyl cannabinoid acids. Phytochemistry, 1984, 23(9), 1909-1912.

[117] Hillig, K.W. Letter to the editor. J. Ind. Hemp, 2002, 7(1), 5-6.

[118] Page, J.E.; Boubakir, Z. Aromatic prenyltransferase from Cannabis. Canadian Patent WO/2011/017798, February 17, 2011. (Also available as U. S. Patent 8884100 B2, November 11, 2014).

[119] Gagne, S.J.; Stout, J.M.; Liu, E.; Boubakir, Z.; Clark, S.M.; Page, J.E. Identification of olivetolic acid cyclase from Cannabis sativa reveals a unique catalytic route to plant polyketides. Proc. Natl. Acad. Sci. USA, 2012, 109, 12811-12816.

[120] Taura, F.; Tanaka, S.; Taguchi, C.; Fukamizu, T.; Tanaka, H.; Shoyama, Y.; Morimoto, S. Characterization of olivetol synthase, a polyketide synthase putatively involved in cannabinoid biosynthetic pathway. FEBS Lett., 2009, 583(12), 2061-2066.

[121] Flores-Sanchez, I.J.; Linthorst, H.J.M.; Verpoorte, R. In silicio expression analysis of PKS genes isolated from Cannabis sativa L. Genet. Mol. Biol., 2010, 33, 703-713.

[122] Raharjo, T.; Widjaja, I.; Roytrakul, S.; Verpoorte, R. Comparative proteomics of Cannabis sativa plant tissues. J. Biomol. Tech., 2004, 15, 97-106.

[123] Raharjo, T.J.; Chang, W.T.; Choi, Y.H.; Peltenburg-Looman, A.M.G.; Verpoorte, R. Olivetol as product of a polyketide synthase in Cannabis sativa L. Plant Sci., 2004, 166, 381-385.

[124] Raharjo, T.J.; Chang, W.T.; Verberne, M.C.; Peltenburg-Looman, A.M.G.; Linthorst, H.J.M.; Verpoorte, R. Cloning and overexpression of a cDNA encoding a polyketide synthase from Cannabis sativa. Plant Physiol. Biochem., 2004, 42, 291-297.

[125] Stout, J.M.; Boubakir, Z.; Ambrose, S.J.; Purves, R.W.; Page, J.E. The hexanoyl-CoA precursor for cannabinoid biosynthesis is formed by an acyl-activating enzyme in Cannabis sativa trichomes. Plant J., 2012, 71, 353-365.

[126] Fellermeier, M.; Eisenreich, W.; Bacher, A.; Zenk, M.H. Biosynthesis of cannabinoids. Eur. J. Biochem., 2001, 268, 15961604.

[127] Hazekamp, A.; Simons, R.; Peltenburg-Looman, A.; Sengers, M.; van Zweden, R.; Verpoorte, R. Preparative isolation of cannabinoids from Cannabis sativa by centrifugal partition chromatography. J. Liq. Chromatogr. Rel. Technol., 2004, 27(15), 2421-2439.

[128] Flockhart, I.; Wheatley, G.W.; Dring, S.; Archer, L. Methods of purifying cannabinoids from plant material. Patent WO/2004/026857, April 1, 2004. (Also available as U.S. patent, 20050266108 A1, December 1, 2004.)

[129] Lehmann, T.; Brenneisen, R. A new chromatographic method for the isolation of (-)- $\Delta$ 9-(trans)-tetrahydrocannabinolic acid A. Phytochem. Anal., 1992, 3(2), 88-90.

[130] Wohlfarth, A.; Mahler, H.; Auwärter, V. Rapid isolation procedure for $\Delta 9$-tetrahydrocannabinolic acid A (THCA) from Cannabis sativa using two flash chromatography systems. J. Chromatogr. B Anal. Technol. Biomed. Life Sci., 2011, 879(28), 3059-3064.

[131] Dussy, F.E.; Hamberg, C.; Luginbühl, M.; Schwerzmann, T.; Briellmann, T.A. Isolation of $\triangle 9$-THCA-A from hemp and analytical aspects concerning the determination of $\triangle 9$-THC in cannabis products. Forensic Sci. Int., 2005, 149(1), 3-10.

[132] Taura, F.; Dono, E.; Sirikantaramas, S.; Yoshimura, K.; Shoyama, Y.; Morimoto, S. Production of delta-1-tetrahydrocannabinolic acid by the biosynthetic enzyme secreted from transgenic Pichia pastoris. Biochem. Biophys. Res. Commun., 2007, 361, 675-680.

[133] Shoyama, Y.; Takeuchi, A.; Taura, F.; Tamada, T.; Adachi, M.; Kuroki, R.; Shoyama, Y.; Morimoto, S. Crystallization of $\Delta 1$ tetrahydrocannabinolic acid (THCA) synthase from Cannabis sativa. Acta Crystallogr. Sect. F Struct. Biol. Cryst. Commun., 2005, 61(8),
799-801.

[134] Happyana, N.; Agnolet, S.; Muntendam, R.; van Dam, A.; Schneider, B.; Kayser, O. Analysis of cannabinoids in laser-microdissected trichomes of medicinal Cannabis sativa using LCMS and cryogenic NMR. Phytochemistry, 2013, 87, 51-59.

[135] Hammond, C.T.; Mahlberg, P.G. Morphology of glandular hairs of Cannabis sativa from scanning electron microscopy. Am. J. Bot., 1973, 60(6), 524-528.

[136] Hammond, C.T.; Mahlberg, P.G. Morphogenesis of capitate glandular hairs of Cannabis sativa (Cannabaceae). Am. J. Bot., 1977, 64(8), 1023-1031.

[137] Dayanandan, P.; Kaufman, P.B. Trichomes of Cannabis sativa L. (Cannabaceae). Am. J. Bot., 1976, 63(5), 578-591.

[138] Mahlberg, P.G.; Kim, E.-S. Accumulation of cannabinoids in glandular trichomes of Cannabis (Cannabaceae). J. Ind. Hemp, 2004, 9(1), 15-36.

[139] Small, E.; Naraine, S.U. Expansion of female sex organs in response to prolonged virginity in Cannabis sativa (marijuana). Genet. Resour. Crop Evol., 2015, 63(2), 339-348.

[140] Hammond, C.T.; Mahlberg, P.G. Ultrastructural development of capitate glandular hairs of Cannabis sativa L. (Cannabaceae). Am. J. Bot., 1978, 65(2), 140-151.

[141] Ohlsson, A.; Abou-Chaar, C.I.; Agurell, S.; Nilsson, I.M.; Olofsson, K.; Sandberg, F. Cannabinoid constituents of male and female Cannabis sativa. UN Bull. Narcotics, 1971, 23, 29-32.

[142] Paris, M.; Boucher, F.; Cosson, L. The constituents of Cannabis sativa pollen. Econ. Bot., 1975, 29(3), 245-253.

[143] Petri, G.; Oroszlán, P.; Fridvalszky, L. Histochemical detection of hemp trichomes and their correlation with the THC content. Acta Biol Hung., 1988, 39(1), 59-73.

[144] Turner, J.C.; Hemphill, J.K.; Mahlberg, P.G. Trichomes and cannabinoid content of developing leaves and bracts of Cannabis sativa L.(Cannabaceae). Am. J. Bot., 1980, 67(10), 1397-1406.

[145] Fischedick, J.; van der Kooy, F.; Verpoorte, R. Cannabinoid receptor 1 binding activity and quantitative analysis of Cannabis sativa L. smoke and vapor. Chem. Pharm. Bull. (Tokyo), 2010, 58(2), 201207.

[146] Hammond, C.T.; Mahlberg, P.G. Phloroglucinol glucoside as a natural constituent of Cannabis sativa. Phytochemistry, 1994, 37(3), 755-756

[147] Mahlberg, P.G.; Kim, E.-S. Cuticle development on glandular trichomes of Cannabis sativa (Cannabaceae). Am. J. Bot., 1991, 78(8), 1113-1122.

[148] Kim, E.-S.; Mahlberg, P.G. Cytochemical localization of cellulase in glandular trichomes of Cannabis (Cannabaceae). J. Plant Biol., 1997, 40(1), 61-66.

[149] Kim, E.-S.; Mahlberg, P.G. Plastid development in disc cells of glandular trichomes of Cannabis (Cannabaceae). Mol. Cells, 1997, 7(3), 352-359.

[150] Solymosi, K.; Schoefs, B. Etioplast and etio-chloroplast formation under natural conditions: The dark side of chlorophyll biosynthesis in angiosperms. Photosynth. Res., 2010, 105, 143-166.

[151] Amelunxen, F. Elektronenmikroskopische Untersuchungen an den Drüsenshuppen von Mentha piperita L. Planta Med., 1965, 13(4), 457-453.

[152] Schnepf, E. Plastidenstrukturen bei Passiflora. Protoplasma, 1961, 54(2), 310-313.

[153] Tollsten, L.; Bergström, L.G. Fragrance chemotypes of Platanthera (Orchidaceae) - the result of adaptation to pollinating moths? Nord. J. Bot., 1993, 13(6), 607-613.

[154] Turner, G.W.; Gershenzon, J.; Croteau, R.B. Development of peltate glandular trichomes of peppermint. Plant Physiol., 2000, 124(2), 665-680.

[155] Vermeer, J.; Peterson, R.L. Glandular trichomes on the inflorescence of Chrysanthemum-morifolium cv dramatic (Compositae). 1. Development and morphology. Can. J. Bot. Can. Bot., 1979, 57(7), 705-713.

[156] Kashina, T.K.; Danilova, M.F. Ultrastructure of glandular hair plastids and nictophylness of Perilla ocymoides L. Russ. J. Plant Physiol., 1993, 40(6), 785-790.

[157] Ascensao, L.; Pais, M.S.S. Secretory trichomes from Artemisia crithmifolia: some ultrastructural aspects. Bull. la Société Bot. Fr. Actual. Bot., 1982, 129(1), 83-87.

[158] Johansson, M.; Walles, B. Functional anatomy of the ovule in broad bean (Vicia faba L.) I. Histogenesis prior to and after pollination. Int J. Plant Sci., 1993, 154(1), 80-89.

[159] Johansson, M.; Walles, B. Functional anatomy of the ovule in broad bean (Vicia faba L.) II. Ultrastructural seed development and nutrient 
pathways. Ann. Bot., 1994, 74(3), 233-244.

[160] Wredle, U.; Walles, B.; Hakman, I. Chromoplasts are formed inVicia faba suspensor cells. Int. J. Plant Sci., 2000, 161(5), 713-719.

[161] Kim, E.-S.; Mahlberg, P.G. Secretory vesicle formation in the secretory cavity of glandular trichomes of Cannabis sativa $\mathrm{L}$. (Cannabaceae). Mol. Cells, 2003, 15(2), 387-395.

[162] Kim, E.-S.; Mahlberg, P.G. Secretory cavity development in glandular trichomes of Cannabis sativa L. (Cannabaceae). Am. J. Bot., 1991, 78(2), 220-229.

[163] Mahlberg, P.G.; Kim, E.-S. Secretory vesicle formation in glandular trichomes of Cannabis sativa (Cannabaceae). Am. J. Bot., 1992, 79(2), 166-173.

[164] Kim, E.-S.; Mahlberg, P.G. Glandular cuticle formation in Cannabis (Cannabaceae). Am. J. Bot., 1995, 82(10), 1207-1214.

[165] Kim, E.-S.; Mahlberg, P.G. Immunochemical localization of tetrahydrocannabinol (THC) in cryofixed glandular trichomes of Cannabis (Cannabaceae). Am. J. Bot., 1997, 84(3), 336-342.

[166] Kim, E.-S.; Mahlberg, P.G. Immunochemical localization of tetrahydrocannabinol (THC) in chemically fixed glandular thrichomes of Cannabis (Cannabaceae). Korean J. Biol. Sci., 1999, 3(2), 215-219.

[167] Mandolino, G.; Ranalli, P. In: Advances in hemp research; Ranalli, P., Ed. Food Products Press (of Haworth Press): New York, 1998; pp. 185-212.

[168] Flores-Sanchez, I.J.; Peč, J.; Fei, J.; Choi, Y.H.; Dušek, J.; Verpoorte, R. Elicitation studies in cell suspension cultures of Cannabis sativa L. J. Biotechnol., 2009, 143, 157-168.

[169] Furr, M.; Mahlberg, P.G. Histochemical analyses of laticifers and glandular trichomes in Cannabis sativa. J. Nat. Prod., 1981, 44(2), 153-159.

[170] Garbacik, E.T.; Korai, R.P.; Frater, E.H.; Korterik, J.P.; Otto, C.; Offerhaus, H.L. In planta imaging of $\Delta^{9}$-tetrahydrocannabinolic acid in Cannabis sativa L. with hyperspectral coherent anti-Stokes Raman scattering microscopy. J. Biomed. Opt., 2013, 18, 046009.

[171] Kelly, J.F. The non-specificity of the Duquenois-Levine field test for marijuana. Open Forensic Sci. J., 2012, 5(1), 4-8.

[172] Yerger, E.H.; Grazzini, R.A.; Hesk, D.; Cox-Foster, D.L.; Craig, R.; Mumma, R.O. A rapid method for isolating glandular trichomes. Plant Physiol., 1992, 99(1), 1-7.

[173] Gershenzon, J.; McCaskill, D.; Rajaonarivony, J.I.; Mihaliak, C.; Karp, F.; Croteau, R. Isolation of secretory cells from plant glandular trichomes and their use in biosynthetic studies of monoterpenes and other gland products. Anal. Biochem., 1992, 200(1), 130-138.

[174] Degenhardt, L.; Chiu, W.T.; Sampson, N.; Kessler, R.C.; Anthony, J.C.; Angermeyer, M.; Bruffaerts, R.; de Girolamo, G.; Gureje, O.; Huang, Y.; Karam, A.; Kostyuchenko, S.; Lepine, J.P.; Mora, M.E.; Neumark, Y.; Ormel, J.H.; Pinto-Meza, A.; Posada-Villa, J.; Stein, D.J.; Takeshima, T.; Wells, J.E. Toward a global view of alcohol, tobacco, cannabis, and cocaine use: findings from the WHO World Mental Health Surveys. PLoS Med., 2008, 5, e141.

[175] Henquet, C.; Kuepper, R. Does cannabidiol protect against the negative effects of THC? Br. J. Psychiatry, 2010, 197(4), 259-260.

[176] Substance Abuse and Mental Health Services Administration Results from the 2010 National Survey on Drug Use and Health. Summary of National Findings. U.S. Department of Health and Human Services: Rockville, MD, 2011.

[177] Volkow, N.D.; Compton, W.M.; Weiss, S.R. Adverse health effects of marijuana use. N. Engl. J. Med., 2014, 370(23), 2219-2227.

[178] Gleason, K.A.; Birnbaum, S.G.; Shukla, A.; Ghose, S. Susceptibility of the adolescent brain to cannabinoids: long-term hippocampal effects and relevance to schizophrenia. Transl. Psychiatry, 2012, 2, e199.

[179] Neto, C.; Fraga, S.; Ramos, E. Consumo de sustancias ilícitas por adolescentes portugueses. Rev. Saude Publica, 2012, 46(5), 808-815.

[180] Hasin, D.S.; Saha, T.D.; Kerridge, B.T.; Goldstein, R.B.; Chou, S.P.; Zhang, H.; Jung, J.; Pickering, R.P.; Ruan, W.J.; Smith, S.M.; Huang, B.; Grant, B.F. Prevalence of Marijuana Use Disorders in the United States Between 2001-2002 and 2012-2013. JAMA Psychiatry, 2015, 72(12), 1235-1242.

[181] Hasin, D.S.; O'Brien, C.P.; Auriacombe, M.; Borges, G.; Bucholz, K.; Budney, A.; Compton, W.M.; Crowley, T.; Ling, W.; Petry, N.M.; Schuckit, M.; Grant, B.F. DSM-5 criteria for substance use disorders: recommendations and rationale. Am. J. Psychiatry, 2013, 170(8), 834-851.

[182] Agrawal, A.; Lynskey, M.T.; Bucholz, K.K.; Kapoor, M.; Almasy, L.; Dick, D.M.; Edenberg, H.J.; Foroud, T.; Goate, A.; Hancock, D.B.; Hartz, S.; Johnson, E.O.; Hesselbrock, V.; Kramer, J.R.; Kuperman, S.; Nurnberger, J.I. Jr; Schuckit, M.; Bierut, L.J. DSM-5 cannabis use disorder: a phenotypic and genomic perspective. Drug Alcohol Depend., 2014, 134, 362-369.

[183] Jaffe, J.H. In: The Pharmacological Basis of Therapeutics. (7th Edition), Gilman, A.G.; Goodman, L.S.; Murad, F., Eds. Macmillan: New York, 1985, pp. 532-581.

[184] Romano, L.L.; Hazekamp, A. Cannabis oil: chemical evaluation of an upcoming cannabis-based medicine. Cannabinoids, 2013, 1(1), 1-11.

[185] Pierre, J.M.; Gandal, M.; Son, M. Cannabis-induced psychosis associated with high potency "wax dabs". Schizophr. Res., 2016, 172(1-3):211-212.

[186] Lemberger, L.; Axelrod, J.; Kopin, I.J. Metabolism disposition of delta-9-tetrahydrocannabinol in man. Pharmacol. Rev., 1971, 23 371-380.

[187] Ohlsson, A.; Lindgren, J.E.; Wahlén, A.; Agurell, S.; Hollister, L.E.; Gillespie, H.K. Plasma delta-9 tetrahydrocannabinol concentrations and clinical effects after oral and intravenous administration and smoking. Clin. Pharmacol. Ther., 1980, 28(3), 409-416.

[188] Lemberger, L.; Martz, R.; Rodda, B.; Forney, R.; Rowe, H. Comparative pharmacology of $\Delta^{9}$-tetrahydrocannabinol and its metabolite, 11-hydroxy- $\Delta^{9}$-tetrahydrocannabinol. J. Clin. Invest., 1973, 52, 2411-2417.

[189] Watanabe, K.; Kijima, T.; Narimatsu, S.; Nishikami, J.; Yamamoto, I.; Yoshimura, H. Comparison of pharmacological effects of tetrahydrocannabinols and their 11-hydroxy-metabolites in mice. Chem. Pharm. Bull., 1990, 38, 2317-2319.

[190] Bisogno, T.; Hanuš, L.; De Petrocellis, L.; Tchilibon, S.; Ponde, D.E.; Brandi, I.; Moriello, A.S.; Davis, J.B.; Mechoulam, R.; Di Marzo, V. Molecular targets for cannabidiol and its synthetic analogues: effect on vanilloid $\mathrm{VR}_{1}$ receptors and on the cellular uptake and enzymatic hydrolysis of anandamide. Br. J. Pharmacol., 2001, 134, 845-852.

[191] De Petrocellis, L.; Ligresti, A.; Moriello, A.S.; Allarà, M.; Bisogno, T.; Petrosino, S.; Stott, C.G.; Di Marzo, V. Effects of cannabinoids and cannabinoid-enriched Cannabis extracts on TRP channels and endocannabinoid metabolic enzymes. Br. J. Pharmacol., 2011, 163(7), 1479-1494

[192] Lindgren, J.E. Quantification of delta 1-tetrahydrocannabinol in tissues and body fluids. Arch. Toxicol. Suppl., 1983, 6, 74-80.

[193] Raikos, N.; Schmid, H.; Nussbaumer, S.; Ambach, L.; Lanz, S.; Längin, A.; König, S.; Roth, N.; Auwärter, V.; Weinmann, W. Determination of $\Delta^{9}$-tetrahydrocannabinolic acid $\mathrm{A}\left(\Delta^{9}\right.$-THCA-A) in whole blood and plasma by LC-MS/MS and application in authentic samples from drivers suspected of driving under the influence of cannabis. Forensic Sci. Int., 2014, 243, 130-136.

[194] Ohlsson, A.; Lindgren, J.E.; Wahlén, A.; Agurell, S.; Hollister, L.E.; Gillespie, H.K. Single dose kinetics of deuterium labelled delta 1tetrahydrocannabinol in heavy and light cannabis users. Biomed. Mass. Spectrom., 1982, 9(1), 6-10.

[195] Johansson, E.; Agurell, S.; Hollister, L.E.; Halldin, M.M. Prolonged apparent half-life of delta 1-tetrahydrocannabinol in plasma of chronic marijuana users. J. Pharm. Pharmacol., 1988, 40(5), 374375.

[196] Dackis, C.A.; Pottash, A.L.C.; Annitto, W.; Gold, M.S. Persistence of urinary marijuana levels after supervised abstinence. American Journal of Psychiatry, 1982, 139(9), 1196-1198.

[197] Ellis, G.M.; Mann, M.A.; Judson, B.A.; Schramm, N.T.; Tashchian, A. Excretion patterns of cannabinoid metabolites after last use in a group of chronic users. Clinical Pharmacology and Therapeutics, 1985, 38, 572-578.

[198] Ashton, C.H. Pharmacology and effects of cannabis: a brief review. Br. J. Psychiatry, 2001, 178, 101-106.

[199] Wall, M.E.; Sadler, B.M.; Brine, D.; Taylor, H.; Perez-Reyes, M. Metabolism, disposition, and kinetics of delta-9-tetrahydrocannabinol in men and women. Clin. Pharmacol. Ther., 1983, 34(3), 352-363.

[200] Watanabe, K.; Yamaori, S.; Funahashi, T.; Kimura, T.; Yamamoto, I. Cytochrome P450 enzymes involved in the metabolism of tetrahydrocannabinols and cannabinol by human hepatic microsomes. Life Sci., 2007, 80(15), 1415-1459.

[201] Ujváry, I.; Grotenhermen, F. 11-Nor-9-carboxy- $\Delta$ 9tetrahydrocannabinol - a ubiquitous yet underresearched cannabinoid. A re-view of the literature. Cannabinoids, 2014, 9(1), 18.

[202] Moosmann, B.; Roth, N.; Auwärter, V. Finding cannabinoids in hair does not prove cannabis consumption. Sci. Rep., 2015, 5, 14906.

[203] Hawks, R.L. In: The Analysis of Cannabinoids in Biological Fluids, National Institute on Drug Abuse Research Monograph No. 42, Hawks, R.L., Ed. U.S. Department of Health and Human Services: Rockville, 1982, pp. 125-137. 
[204] Gross, S.J.; Worthy, T.E.; Nerder, L., Zimmermann, E.G.; Soares, J.R.; Lomax, P. Detection of recent cannabis use by saliva delta 9THC radioimmunoassay. J. Anal. Toxicol., 1985, 9(1), 1-5.

[205] Cohen, S. The 94-day cannabis study. Ann. N. Y. Acad. Sci., 1976, 282, 211-220.

[206] Jones, R.T. In: Marijuana '84: Proceedings of the Oxford Symposium on Cannabis, Harvey, D.J.; Paton, W.; Nahas G.G., Eds. IRL Press: Oxford, 1984.

[207] Maykut, M.O. Health Consequences of Acute and Chronic Marihuana Use, Pergamon Press: Oxford, 1984.

[208] Foltin, R.W.; Fischman, M.W.; Byrne, M.F. Effects of smoked marijuana on food intake and body weight of humans living in a residential laboratory. Appetite, 1988, 11, 1-14.

[209] Huber, G.L.; Griffith, D.L.; Langsjoen, P.M. In: Marijuana: An International Research Report. National Campaign Against Drug Abuse Monograph Number 7, Chesher, G.; Consroe, P.; Musty, R., Eds. Australian Government Publishing Service: Canberra, 1988.

[210] Rodondi, N.; Pletcher, M.J.; Liu, K.; Hulley, S.B.; Sidney, S. Coronary Artery Risk Development in Young Adults (CARDIA) Study. Marijuana use, diet, body mass index, and cardiovascular risk factors (from the CARDIA study). Am. J. Cardiol., 2006, 98(4), 478484.

[211] Smith, D.E. Acute and chronic toxicity of marijuana. J. Psychedelic Drugs, 1968, 2, 37-47.

[212] Tart, C. Marijuana intoxication: Common experiences. Nature, 1970, 226, 701-704.

[213] Weil, A. Adverse reactions to marihuana. New England Journal of Medicine, 1970, 282, 997-1000.

[214] Thomas, H. Psychiatric symptoms in cannabis users. Br. J. Psychiatry, 1993, 163, 141-149.

[215] D'Souza, D.C.; Perry, E.; MacDougall, L.; Ammerman, Y.; Cooper, T.; Wu, Y.T.; Braley, G.; Gueorguieva, R.; Krystal, J.H. The psychotomimetic effects of intravenous delta-9-tetrahydrocannabinol in healthy individuals: Implications for psychosis. Neuropsychopharmacol., 2004, 29, 1558-1572.

[216] D'Souza, D.C.; Ranganathan, M.; Braley, G.; Gueorguieva, R.; Zimolo, Z.; Cooper, T.; Perry, E.; Krystal, J. Blunted psychotomimetic and amnestic effects of delta-9tetrahydrocannabinol in frequent users of cannabis. Neuropsychopharmacol., 2008, 33(10), 2505-2516.

[217] Köfalvi, A.; Fritzsche, M. In: Cannabinoids and the Brain; Köfalvi, A., Ed. Springer US, 2008, pp. 485-528.

[218] Sewell, R.A.; Schnakenberg, A.; Elander, J.; Radhakrishnan, R.; Williams, A.; Skosnik, P.D.; Pittman, B.; Ranganathan, M.; D'Souza, D.C. Acute effects of THC on time perception in frequent and infrequent cannabis users. Psychopharmacology (Berl)., 2013, 226(2), 401-413.

[219] Smith, P.B.; Compton, D.R.; Welch, S.P.; Razdan, R.K.; Mechoulam, R.; Martin, B.R. The pharmacological activity of anandamide, a putative endogenous cannabinoid, in mice. J. Pharmacol. Exp. Ther., 1994, 270(1), 219-227.

[220] Varvel, S.A.; Bridgen, D.T.; Tao, Q.; Thomas, B.F.; Martin, B.R.; Lichtman, A.H. Delta ${ }^{9}$-tetrahydrocannabinol accounts for the antinociceptive, hypothermic, and cataleptic effects of marijuana in mice. J. Pharmacol. Exp. Ther., 2005, 314, 329-337.

[221] Lawrence, D.K.; Pertwee, R.G. Brain levels of $\Delta^{1}$ tetrahydrocannabinol and its metabolites in mice tolerant to the hypothermic effect of delta1-tetrahydrocannabinol. $\mathrm{Br} . \mathrm{J}$ Pharmacol., 1973, 49(2), 373-375.

[222] Oviedo, A.; Glowa, J.; Herkenham, M. Chronic cannabinoid administration alters cannabinoid receptor binding in rat brain: a quantitative autoradiographic study. Brain Res., 1993, 616(1-2), 293302.

[223] Tsou, K.; Patrick, S.L.; Walker, J.M. Physical withdrawal in rats tolerant to delta 9-tetrahydrocannabinol precipitated by a cannabinoid receptor antagonist. Eur. J .Pharmacol., 1995, 280(3), R13-15.

[224] Wu, X.; French, E.D. Effects of chronic $\Delta^{9}$-tetrahydrocannabinol on rat midbrain dopamine neurons: an electrophysiological assessment. Neuropharmacology, 2000, 39(3), 391-398.

[225] Perez-Reyes, M.; White, W.R.; McDonald, S.A.; Hicks, R.E.; Jeffcoat, A.R.; Cook, CE. The pharmacologic effects of daily marijuana smoking in humans. Pharmacol. Biochem. Behav., 1991, 40(3), 691-694.

[226] Hart, C.L.; van Gorp, W.; Haney, M.; Foltin, R.W.; Fischman, M.W. Effects of acute smoked marijuana on complex cognitive performance. Neuropsychopharmacol., 2001, 25, 757-765.

[227] Stone, J.M.; Morrison, P.D.; Nottage, J.; Bhattacharyya, S.; Feilding, A.; McGuire, P.K. Delta-9-tetrahydrocannabinol disruption of time perception and of self-timed actions. Pharmacopsychiatry, 2010, 43, 236-237.

[228] Gorelick, D.A.; Goodwin, R.S.; Schwilke, E.; Schroeder, J.R.; Schwope, D.M.; Kelly, D.L.; Ortemann-Renon, C.; Bonnet, D.; Huestis, M.A. Around-the-clock oral THC effects on sleep in male chronic daily cannabis smokers. Am. J. Addict., 2013, 22(5), 510-514.

[229] Dudok, B.; Barna, L.; Ledri, M.; Szabó, S.I.; Szabadits, E.; Pintér, B.; Woodhams, S.G.; Henstridge, C.M.; Balla, G.; Nyilas, R.; Varga, C.; Lee, S.H.; Matolcsi, M.; Cervenak, J.; Kacskovics, I.; Watanabe, M.; Sagheddu, C.; Melis, M.; Pistis, M.; Soltesz, I.; Katona, I. Cellspecific STORM super-resolution imaging reveals nanoscale organization of cannabinoid signaling. Nat. Neurosci., 2015, 18(1), 75-86.

[230] Rinaldi-Carmona, M.; Barth, F.; Héaulme, M.; Shire, D.; Calandra, B.; Congy, C.; Martinez, S.; Maruani, J.; Néliat, G.; Caput, D.; Ferrara, P.; Soubrié, P.; Brelière, J.C.; Le Fur, G. SR141716A, a potent and selective antagonist of the brain cannabinoid receptor. FEBS Lett., 1994, 350, 240-244.

[231] Leterrier, C.; Lainé, J.; Darmon, M.; Boudin, H.; Rossier, J.; Lenkei, Z. Constitutive activation drives compartment-selective endocytosis and axonal targeting of type 1 cannabinoid receptors. J. Neurosci., 2006, 26(12), 3141-3153.

[232] Meye, F.J.; Ramakers, G.M.; Adan, R.A. The vital role of constitutive GPCR activity in the mesolimbic dopamine system. Transl. Psychiatry., 2014, 4, e361.

[233] Aceto, M.D.; Scates, S.M.; Lowe, J.A.; Martin, B.R. Dependence on delta 9-tetrahydrocannabinol: studies on precipitated and abrupt withdrawal. J. Pharmacol. Exp. Ther., 1996, 278(3), 1290-1295.

[234] Canas, P.M.; Duarte, J.M.; Rodrigues, R.J.; Köfalvi, A.; Cunha, R.A. Modification upon aging of the density of presynaptic modulation systems in the hippocampus. Neurobiol. Aging, 2009, 30(11), 18771884.

[235] Feliszek, M.; Bindila, L.; Lutz, B.; Zimmer, A.; Bilkei-Gorzo, A.; Schlicker, E. Lack of hippocampal CB1 receptor desensitization by $\Delta(9)$-tetrahydrocannabinol in aged mice and by low doses of JZL 184. Naunyn. Schmiedebergs Arch. Pharmacol., 2016, 389(6), 603612.

[236] Hall, W. The mental health risks of adolescent cannabis use. PLoS Med., 2006, 3, 159-162.

[237] Balter, R.E.; Cooper, Z.D.; Haney, M. Novel Pharmacologic Approaches to Treating Cannabis Use Disorder. Curr. Addict. Rep., 2014, 1(2), 137-143.

[238] Hoch, E.; Bonnetn, U.; Thomasius, R.; Ganzer, F.; HavemannReinecke, U.; Preuss, U.W. Risks associated with the non-medicinal use of cannabis. Dtsch. Arztebl. Int., 2015, 112(16), 271-278.

[239] Sañudo-Peña, M.C.; Force, M.; Tsou, K.; McLemore, G.; Roberts, L.; Walker, J.M. Dopaminergic system does not play a major role in the precipitated cannabinoid withdrawal syndrome. Zhongguo Yao Li Xue Bao, 1999, 20(12), 1121-1124.

[240] McClure, E.A.; Sonne, S.C.; Winhusen, T.; Carroll, K.M.; Ghitza, U.E.; McRae-Clark, A.L.; Matthews, A.G.; Sharma, G.; van Veldhuisen, P.; Vandrey, R.G.; Levin, F.R.; Weiss, R.D.; Lindblad, R.; Allen, C.; Mooney, L.J.; Haynes, L.; Brigham, G.S.; Sparenborg, S.; Hasson, A.L.; Gray, K.M. Achieving cannabis cessation -evaluating $\mathrm{N}$-acetylcysteine treatment (ACCENT): design and implementation of a multi-site, randomized controlled study in the National Institute on Drug Abuse Clinical Trials Network. Contemp. Clin. Trials, 2014, 39(2), 211-223.

[241] Ehlers, C.L.; Gizer, I.R.; Vieten, C.; Gilder, D.A.; Stouffer, G.M.; Lau, P.; Wilhelmsen, K.C. Cannabis dependence in the San Francisco Family Study: age of onset of use, DSM-IV symptoms, withdrawal, and heritability. Addict. Behav., 2010, 35(2), 102-110.

[242] Hernandez-Avila, C.A.; Rounsaville, B.J.; Kranzler, H.R. Opioidcannabis-and alcohol-dependent women show more rapid progression to substance abuse treatment. Drug Alcohol Depend., 2004, 74(3), 265-272.

[243] Schepis, T.S.; Desai, R.A.; Cavallo, D.A.; Smith, A.E.; McFetridge, A.; Liss, T.B.; Potenza, M.N.; Krishnan-Sarin, S. Gender differences in adolescent marijuana use and associated psychosocial characteristics. J. Addict. Med., 2011, 5(1), 65-73.

[244] Westermeyer, J.; Boedicker, A.E. Course, severity, and treatment of substance abuse among women versus men. Am. J. Drug Alcohol Abuse, 2000, 26(4), 523-535.

[245] Fogel, J.S.; Kelly, T.H.; Westgate, P.M.; Lile, J.A. Sex differences in the subjective effects of oral $\Delta^{9}$-THC in cannabis users. Pharmacol. Biochem. Behav., 2016, in press, doi:10.1016/j.pbb.2016.01.007.

[246] Moir, D.; Rickert, W.S.; Levasseur, G.; Larose, Y.; Maertens, R.; White, P.; Desjardins, S. A comparison of mainstream and sidestream 
marijuana and tobacco cigarette smoke produced under two machine smoking conditions. Chem. Res. Toxicol., 2008, 21(2), 494-502.

[247] Cohen, S. Adverse effects of marijuana: selected issues. Ann. N. Y. Acad. Sci., 1981, 362, 119-124.

[248] Tashkin, D.P. Airway effects of marijuana, cocaine, and other inhaled illicit agents. Curr. Opin. Pulm. Med., 2001, 7(2), 43-61.

[249] Owen, K.P.; Sutter, M.E.; Albertson, T.E. Marijuana: respiratory tract effects. Clin. Rev. Allergy Immunol., 2014, 46(1), 65-81.

[250] de Carvalho, M.F.; Dourado, M.R.; Fernandes, I.B.; Araújo, C.T.; Mesquita, A.T.; Ramos-Jorge, M.L. Head and neck cancer among marijuana users: A meta-analysis of matched case-control studies. Arch. Oral Biol., 2015, 60(12), 1750-1755.

[251] Geller, T.; Loftis, L.; Brink, D.S. Cerebellar infarction in adolescent males associated with acute marijuana use. Pediatrics, 2004, 113(4), e365-370.

[252] Mateo, I.; Pinedo, A.; Gomez-Beldarrain, M.; Basterretxea, J.M.; Garcia-Monco, J.C. Recurrent stroke associated with cannabis use. J. Neurol .Neurosurg. Psychiatry, 2005, 76, 435-437.

[253] Wolff, V.; Lauer, V.; Rouyer, O.; Sellal, F.; Meyer, N.; Raul, J.S.; Sabourdy, C.; Boujan, F.; Jahn, C.; Beaujeux, R.; Marescaux, C. Cannabis use, ischemic stroke, and multifocal intracranial vasoconstriction: a prospective study in 48 young patients. Stroke, 2011, 42, 1778-1780.

[254] Singh, N.; Pan, Y.; Muengtaweeponsa, S.; Geller, T.J.; Cruz-Flores, S. Cannabis-related stroke: case series and review of the literature. $J$. Stroke Cerebrovasc. Dis., 2012, 21, 555-560.

[255] Bachs, L.; Mørland, H. Acute cardiovascular fatalities following cannabis use. Forensic Sci. Int., 2001, 124(2-3), 200-203.

[256] Kayar, Y.; Eroğlu, H.; Pamukçu, O.; Cetin, H.; Koçaş, O.; Atçı, M.; Turk, J. Cannabinoid-induced acute pancreatitis. Gastroenterol., 2014, 25(3), 335-336.

[257] Clark, B.C.; Georgekutty, J.; Berul, C.I. Myocardial ischemia secondary to synthetic cannabinoid (K2) use in pediatric patients. $J$. Pediatr., 2015, 167(3), 757-761.

[258] Penner, E.A.; Buettner, H.; Mittleman, M.A. The impact of marijuana use on glucose, insulin, and insulin resistance among US adults. Am. J. Med., 2013, 126(7), 583-589.

[259] Rajavashisth, T.B.; Shaheen, M.; Norris, K.C.; Pan, D.; Sinha, S.K.; Ortega, J.; Friedman, T.C. Decreased prevalence of diabetes in marijuana users: cross-sectional data from the National Health and Nutrition Examination Survey (NHANES) III. BMJ Open, 2012, 2, e000494.

[260] Kunos, G.; Osei-Hyiaman, D.; Liu, J.; Godlewski, G.; Bátkai, S. Endocannabinoids and the control of energy homeostasis. J. Biol. Chem., 2008, 283(48), 33021-33025.

[261] Matias, I.; Marzo, V.D.; Köfalvi, A. In: Cannabinoids and the Brain; Köfalvi, A., Ed. Springer US, 2008, pp. 277-316.

[262] Tam, J.; Vemuri, V.K.; Liu, J.; Bátkai, S.; Mukhopadhyay, B.; Godlewski, G.; Osei-Hyiaman, D.; Ohnuma, S.; Ambudkar, S.V.; Pickel, J.; Makriyannis, A.; Kunos, G. Peripheral $\mathrm{CB}_{1}$ cannabinoid receptor blockade improves cardiometabolic risk in mouse models of obesity. J. Clin. Invest., 2010, 120(8), 2953-2966.

[263] Topol, E.J.; Bousser, M.G.; Fox, K.A.; Creager, M.A.; Despres, J.P.; Easton, J.D.; Hamm, C.W.; Montalescot, G.; Steg, P.G.; Pearson, T.A.; Cohen, E.; Gaudin, C.; Job, B.; Murphy, J.H.; Bhatt, D.L.; CRESCENDO Investigators. Rimonabant for prevention of cardiovascular events (CRESCENDO): a randomised, multicentre, placebo-controlled trial. Lancet, 2010, 376(9740), 517-523.

[264] Mechoulam, R.; Hanuš, L.O.; Pertwee, R.; Howlett, A.C. Early phytocannabinoid chemistry to endocannabinoids and beyond. Nat. Rev. Neurosci., 2014, 15(11), 757-764.

[265] Di Marzo, V.; Fontana, A.; Cadas, H.; Schinelli, S.; Cimino, G.; Schwartz, J.C.; Piomelli, D. Formation and inactivation of endogenous cannabinoid anandamide in central neurons. Nature, 1994, 372(6507), 686-691.

[266] Di Marzo, V.; Fontana, A. Anandamide, an endogenous cannabinomimetic eicosanoid: 'killing two birds with one stone'. Prostaglandins Leukot. Essent. Fatty Acids, 1995, 53, 1-11.

[267] Izzo, A.A.; Borrelli, F.; Capasso, R.; Di Marzo, V.; Mechoulam, R. Non-psychotropic plant cannabinoids: new therapeutic opportunities from an ancient herb. Trends Pharmacol. Sci., 2009, 30(10), 515-527.

[268] Gertsch, J.; Pertwee, R.G.; Di Marzo, V. Phytocannabinoids beyond the Cannabis plant - do they exist? Br. J .Pharmacol., 2010, 160(3), 523-529.

[269] Ligresti, A.; Villano, R.; Allarà, M.; Ujváry, I.; Di Marzo, V. Kavalactones and the endocannabinoid system: the plant-derived yangonin is a novel $\mathrm{CB}_{1}$ receptor ligand. Pharmacol. Res., 2012, 66(2), 163-169.
[270] Rempel, V.; Fuchs, A.; Hinz, S.; Karcz, T.; Lehr, M.; Koetter, U.; Müller, C.E. Magnolia extract, magnolol, and metabolites: Activation of cannabinoid CB2 receptors and blockade of the related GPR55. ACS Med. Chem. Lett., 2012, 4(1), 41-45.

[271] Gérard, C.; Mollereau, C.; Vassart, G.; Parmentier, M. Nucleotide sequence of a human cannabinoid receptor cDNA. Nucleic. Acids Res., 1990, 18, 7142

[272] Gérard, C.M.; Mollereau, C.; Vassart, G.; Parmentier, M. Molecular cloning of a human cannabinoid receptor which is also expressed in testis. Biochem. J., 1991, 279, 129-134.

[273] Matsuda, L.A.; Lolait, S.J.; Brownstein, M.J.; Young, A.C.; Bonner, T.I. Structure of a cannabinoid receptor and functional expression of the cloned cDNA. Nature, 1990, 346, 561-564.

[274] Devane, W.A.; Hanuš, L.; Breuer, A.; Pertwee, R.G.; Stevenson, L.A.; Griffin, G.; Gibson, D.; Mandelbaum, A.; Etinger, A.; Mechoulam, R. Isolation and structure of a brain constituent that binds to the cannabinoid receptor. Science, 1992, 258, 1946-1949.

[275] Felder, C.C.; Briley, E.M.; Axelrod, J.; Simpson, J.T.; Mackie, K. Devane, W.A. Anandamide, an endogenous cannabimimetic eicosanoid, binds to the cloned human cannabinoid receptor and stimulates receptor-mediated signal transduction. Proc. Natl. Acad. Sci. USA, 1993, 90(16), 7656-7660.

[276] Munro, S.; Thomas, K.L.; Abu-Shaar, M. Molecular characterization of a peripheral receptor for cannabinoids. Nature, 1993, 365, 61-65.

[277] Mechoulam, R.; Ben-Shabat, S.; Hanuš, L.; Ligumsky, M.; Kaminski, N.E.; Schatz, A.R.; Gopher, A.; Almog, S.; Martin, B.R.; Compton, D.R.; Pertwee, R.G.; Griffin, G.; Bayewitch, M.; Barg, J.; Vogel, Z. Identification of an endogenous 2-monoglyceride, present in canine gut, that binds to cannabinoid receptors. Biochem. Pharmacol., 1995 50, 83-90.

[278] Sugiura, T.; Kondo, S.; Sukagawa, A.; Nakane, S.; Shinoda, A.; Itoh, K.; Yamashita, A.; Waku, K. 2-Arachidonoylglycerol: a possible endogenous cannabinoid receptor ligand in brain. Biochem. Biophys. Res. Commun., 1995, 215, 89-97.

[279] Stella, N.; Schweitzer, P.; Piomelli, D. A second endogenous cannabinoid that modulates long-term potentiation. Nature, 1997, 388(6644), 773-778.

[280] Bayewitch, M.; Avidor-Reiss, T.; Levy, R.; Barg, J.; Mechoulam, R.; Vogel, Z. The peripheral cannabinoid receptor: adenylate cyclase inhibition and G protein coupling. FEBS Lett., 1995, 375, 143-147.

[281] Felder, C.C.; Nielsen, A.; Briley, E.M.; Palkovits, M.; Priller, J.; Axelrod, J.; Nguyen, D.N.; Richardson, J.M.; Riggin, R.M.; Koppel, G.A.; Paul, S.M.; Becker, G.W. Isolation and measurement of the endogenous cannabinoid receptor agonist, anandamide, in brain and peripheral tissues of human and rat. FEBS Lett., 1996, 393(2-3), 231235.

[282] Rinaldi-Carmona, M.; Calandra, B.; Shire, D.; Bouaboula, M.; Oustric, D.; Barth, F.; Casellas, P.; Ferrara, P.; Le Fur, G. Characterization of two cloned human $\mathrm{CB}_{1}$ cannabinoid receptor isoforms. J. Pharmacol. Exp. Ther., 1996, 278, 871-878.

[283] Sugiura, T.; Kodaka, T.; Nakane, S.; Miyashita, T.; Kondo, S.; Suhara, Y.; Takayama, H.; Waku, K.; Seki, C.; Baba, N.; Ishima, Y. Evidence that the cannabinoid $\mathrm{CB}_{1}$ receptor is a 2 arachidonoylglycerol receptor. Structure-activity relationship of 2arachidonoylglycerol, ether-linked analogues, and related compounds. J. Biol. Chem., 1999, 274(5), 2794-2801.

[284] Sugiura, T.; Kondo, S.; Kishimoto, S.; Miyashita, T.; Nakane, S.; Kodaka, T.; Suhara, Y.; Takayama, H.; Waku, K. Evidence that 2arachidonoylglycerol but not N-palmitoylethanolamine or anandamide is the physiological ligand for the cannabinoid $\mathrm{CB}_{2}$ receptor. Comparison of the agonistic activities of various cannabinoid receptor ligands in HL-60 cells. J. Biol. Chem., 2000, 275(1), 605-612.

[285] Piomelli, D. The molecular logic of endocannabinoid signalling. Nat. Rev. Neurosci., 2003, 4, 873-884.

[286] Katona, I.; Freund, T.F. Multiple functions of endocannabinoid signaling in the brain. Annu. Rev. Neurosci., 2012, 35, 529-558.

[287] Placzek, E.A.; Okamoto, Y.; Ueda, N.; Barker, E.L. Membrane microdomains and metabolic pathways that define anandamide and 2 arachidonyl glycerol biosynthesis and breakdown. Neuropharmacology, 2008, 55(7), 1095-1104.

[288] Sugiura, T. In: Cannabinoids and the Brain; Köfalvi, A., Ed. Springer US, 2008, pp. 15-30.

[289] Nyilas, R.; Dudok, B.; Urbán, G.M.; Mackie, K.; Watanabe, M.; Cravatt, B.F.; Freund, T.F.; Katona, I. Enzymatic machinery for endocannabinoid biosynthesis associated with calcium stores in glutamatergic axon terminals. J. Neurosci., 2008, 28(5), 1058-1063. 
[290] Pickel, V.M.; Shobin, E.T.; Lane, D.A.; Mackie, K. Cannabinoid-1 receptors in the mouse ventral pallidum are targeted to axonal profiles expressing functionally opposed opioid peptides and contacting $\mathrm{N}$ acylphosphatidylethanolamine-hydrolyzing phospholipase D terminals. Neuroscience, 2012, 227, 10-21.

[291] Farooqui, A.A.; Rammohan, K.W.; Horrocks, L.A. Isolation, characterization, and regulation of diacylglycerol lipases from the bovine brain. Ann. N. Y. Acad. Sci., 1989, 559, 25-36.

[292] Bisogno, T.; Melck, D.; De Petrocellis, L.; Di Marzo, V. Phosphatidic acid as the biosynthetic precursor of the endocannabinoid 2arachidonoylglycerol in intact mouse neuroblastoma cells stimulated with ionomycin. J. Neurochem., 1999, 72(5), 2113-2119.

[293] Gao, Y.; Vasilyev, D.V.; Goncalves, M.B.; Howell, F.V.; Hobbs, C.; Reisenberg, M.; Shen, R.; Zhang, M.Y.; Strassle, B.W.; Lu, P.; Mark, L.; Piesla, M.J.; Deng, K.; Kouranova, E.V.; Ring, R.H.; Whiteside, G.T.; Bates, B.; Walsh, F.S.; Williams, G.; Pangalos, M.N.; Samad, T.A.; Doherty, P. Loss of retrograde endocannabinoid signaling and reduced adult neurogenesis in diacylglycerol lipase knock-out mice. J. Neurosci., 2010, 30(6), 2017-2024.

[294] Tanimura, A.; Yamazaki, M.; Hashimotodani, Y.; Uchigashima, M.; Kawata, S.; Abe, M.; Kita, Y.; Hashimoto, K.; Shimizu, T.; Watanabe, M.; Sakimura, K.; Kano, M. The endocannabinoid 2arachidonoylglycerol produced by diacylglycerol lipase alpha mediates retrograde suppression of synaptic transmission. Neuron, 2010, 65(3), 320-327.

[295] Higgs, H.N.; Glomset, J.A. Identification of a phosphatidic acidpreferring phospholipase A1 from bovine brain and testis. Proc. Natl. Acad. Sci. USA, 1994, 91, 9574-9578.

[296] Nakane, S.; Oka, S.; Arai, S.; Waku, K.; Ishima, Y.; Tokumura, A.; Sugiura, T. 2-Arachidonoyl-sn-glycero-3-phosphate, an arachidonic acid-containing lysophosphatidic acid: occurrence and rapid enzymatic conversion to 2-arachidonoyl-sn-glycerol, a cannabinoid receptor ligand, in rat brain. Arch. Biochem. Biophys., 2002, 402, 5158.

[297] Di Marzo, V.; Melck, D.; Bisogno, T.; De Petrocellis, L. Endocannabinoids: endogenous cannabinoid receptor ligands with neuromodulatory action. Trends Neurosci., 1998, 21, 521-528.

[298] Maejima, T.; Oka, S.; Hashimotodani, Y.; Ohno-Shosaku, T.; Aiba, A.; Wu, D.; Waku, K.; Sugiura, T.; Kano, M. Synaptically driven endocannabinoid release requires $\mathrm{Ca}^{2+}$-assisted metabotropic glutamate receptor subtype 1 to phospholipase $C \beta 4$ signaling cascade in the cerebellum. J. Neurosci., 2005, 25, 6826-6835.

[299] Castillo, P.E.; Younts, T.J.; Chávez, A.E.; Hashimotodani, Y. Endocannabinoid signaling and synaptic function. Neuron, 2012, 76(1), 70-81.

[300] Turu, G.; Várnai, P.; Gyombolai, P.; Szidonya, L.; Offertaler, L.; Bagdy, G.; Kunos, G.; Hunyady, L. Paracrine transactivation of the CB1 cannabinoid receptor by AT1 angiotensin and other Gq/11 protein-coupled receptors. J. Biol. Chem., 2009, 284(25), 1691416921.

[301] Min, R.; Di Marzo, V.; Mansvelder, H.D. DAG lipase involvement in depolarization-induced suppression of inhibition: does endocannabinoid biosynthesis always meet the demand? Neuroscientist, 2010, 16(6), 608-613.

[302] Alger, B.E.; Kim, J. Supply and demand for endocannabinoids. Trends Neurosci., 2011, 34, 304-315.

[303] Hashimotodani, Y.; Ohno-Shosaku, T.; Tanimura, A.; Kita, Y.; Sano, Y.; Shimizu, T.; Di Marzo, V.; Kano, M. Acute inhibition of diacylglycerol lipase blocks endocannabinoid-mediated retrograde signalling: evidence for on-demand biosynthesis of 2arachidonoylglycerol. J. Physiol., 2013, 591(19), 4765-4776.

[304] Cravatt, B.F.; Giang, D.K.; Mayfield, S.P.; Boger, D.L.; Lerner, R.A.; Gilula, N.B. Molecular characterization of an enzyme that degrades neuromodulatory fatty-acid amides. Nature, 1996, 384(6604), 83-87.

[305] Wei, B.Q.; Mikkelsen, T.S.; McKinney, M.K.; Lander, E.S.; Cravatt, B.F. A second fatty acid amide hydrolase with variable distribution among placental mammals. J. Biol. Chem., 2006, 281(48), 3656936578.

[306] McKinney, M.K.; Cravatt, B.F. Structure and function of fatty acid amide hydrolase. Annu. Rev. Biochem., 2005, 74, 411-432.

[307] Ahn, K.; Johnson, D.S.; Cravatt, B.F. Fatty acid amide hydrolase as a potential therapeutic target for the treatment of pain and CNS disorders. Expert Opin. Drug Discov., 2009, 4, 763-784.

[308] Panlilio, L.V.; Justinova, Z.; Goldberg, S.R. Inhibition of FAAH and activation of PPAR: new approaches to the treatment of cognitive dysfunction and drug addiction. Pharmacol. Ther., 2013, 138(1), 84102.
[309] Sałaga, M.; Sobczak, M.; Fichna, J. Inhibition of fatty acid amide hydrolase (FAAH) as a novel therapeutic strategy in the treatment of pain and inflammatory diseases in the gastrointestinal tract. Eur. J. Pharm. Sci., 2014, 52, 173-179.

[310] Fowler, C.J. The potential of inhibitors of endocannabinoid metabolism as anxiolytic and antidepressive drugs -- A practical view. Eur. Neuropsychopharmacol., 2015, 25(6), 749-762.

[311] Fu, J.; Bottegoni, G.; Sasso, O.; Bertorelli, R.; Rocchia, W.; Masetti, M.; Guijarro, A.; Lodola, A.; Armirotti, A.; Garau, G.; Bandiera, T.; Reggiani, A.; Mor, M.; Cavalli, A.; Piomelli, D. A catalytically silent FAAH-1 variant drives anandamide transport in neurons. Nat. Neurosci., 2011, 15(1), 64-69.

[312] Leung, K.; Elmes, M.W.; Glaser, S.T.; Deutsch, D.G.; Kaczocha, M. Role of FAAH-like anandamide transporter in anandamide inactivation. PLoS ONE, 2013, 8(11), e79355.

[313] Dinh, T.P.; Carpenter, D.; Leslie, F.M.; Freund, T.F.; Katona, I.; Sensi, S.L.; Kathuria, S.; Piomelli, D. Brain monoglyceride lipase participating in endocannabinoid inactivation. Proc. Natl. Acad. Sci. USA, 2002, 99(16), 10819-10824.

[314] Dinh, T.P.; Freund, T.F.; Piomelli, D. A role for monoglyceride lipase in 2-arachidonoylglycerol inactivation. Chem. Phys. Lipids, 2002, 121(1-2), 149-158.

[315] Hashimotodani, Y.; Ohno-Shosaku, T.; Kano, M. Presynaptic monoacylglycerol lipase activity determines basal endocannabinoid tone and terminates retrograde endocannabinoid signaling in the hippocampus. J. Neurosci., 2007, 27, 1211-1219.

[316] Pan, B.; Wang, W.; Long, J.Z.; Sun, D.; Hillard, C.J.; Cravatt, B.F.; Liu, Q-S. Blockade of 2-arachidonoylglycerol hydrolysis by selective monoacylglycerol lipase inhibitor 4-nitrophenyl 4(dibenzo[d][1,3]dioxol-5-yl(hydroxy)methyl)piperidine-1carboxylate (JZL184) enhances retrograde endocannabinoid signaling. J. Pharmacol. Exp. Ther., 2009, 331, 591-597.

[317] Straiker, A.; Hu, S.S.; Long, J.Z.; Arnold, A.; Wager-Miller, J.; Cravatt, B.F.; Mackie, K. Monoacylglycerol lipase limits the duration of endocannabinoid-mediated depolarization-induced suppression of excitation in autaptic hippocampal neurons. Mol. Pharmacol., 2009, 76, 1220-1227.

[318] Chanda, P.K.; Gao, Y.; Mark, L.; Btesh, J.; Strassle, B.W.; Lu, P.; Piesla, M.J.; Zhang, M.Y.; Bingham, B.; Uveges, A.; Kowal, D.; Garbe, D.; Kouranova, E.V.; Ring, R.H.; Bates, B.; Pangalos, M.N.; Kennedy, J.D.; Whiteside, G.T.; Samad, T.A. Monoacylglycerol lipase activity is a critical modulator of the tone and integrity of the endocannabinoid system. Mol. Pharmacol., 2010, 78, 996-1003.

[319] Schlosburg, J.E.; Blankman, J.L.; Long, J.Z.; Nomura, D.K.; Pan, B.; Kinsey, S.G.; Nguyen, P.T.; Ramesh, D.; Booker, L.; Burston, J.J.; Thomas, E.A.; Selley, D.E.; Sim-Selley, L.J.; Liu, Q.S.; Lichtman, A.H.; Cravatt, B.F. Chronic monoacylglycerol lipase blockade causes functional antagonism of the endocannabinoid system. Nat Neurosci., 2010, 13, 1113-1119.

[320] Zhong, P.; Pan, B.; Gao, X.P.; Blankman, J.L.; Cravatt, B.F.; Liu, Q.S. Genetic deletion of monoacylglycerol lipase alters endocannabinoid-mediated retrograde synaptic depression in the cerebellum. J. Physiol., 2011, 589(20), 4847-4855.

[321] Muccioli, G.G.; Xu, C.; Odah, E.; Cudaback, E.; Cisneros, J.A.; Lambert, D.M.; López Rodríguez, M.L.; Bajjalieh, S.; Stella, N. Identification of a novel endocannabinoid-hydrolyzing enzyme expressed by microglial cells. J. Neurosci., 2007, 27(11), 2883-2889.

[322] Blankman, J.L.; Simon, G.M.; Cravatt, B.F. A comprehensive profile of brain enzymes that hydrolyze the endocannabinoid 2arachidonoylglycerol. Chem. Biol., 2007, 14, 1347-1356.

[323] Savinainen, J.R.; Saario, S.M.; Laitinen, J.T. The serine hydrolases MAGL, ABHD6 and ABHD12 as guardians of 2arachidonoylglycerol signalling through cannabinoid receptors. Acta Physiol. (Oxf)., 2012, 204(2), 267-276.

[324] Murataeva, N.; Straiker, A.; Mackie, K. Parsing the players: 2arachidonoylglycerol synthesis and degradation in the CNS. Br. J .Pharmacol., 2014, 171(6), 1379-1391.

[325] Kozak, K.R.; Gupta, R.A.; Moody, J.S.; Ji, C.; Boeglin, W.E.; DuBois, R.N.; Brash, A.R.; Marnett, L.J. 15-Lipoxygenase metabolism of 2-arachidonylglycerol. Generation of a peroxisome proliferator-activated receptor alpha agonist. J. Biol. Chem., 2002, 277, 23278-23286.

[326] Glaser, S.T.; Kaczocha, M. Cyclooxygenase-2 mediates anandamide metabolism in the mouse brain. J. Pharmacol. Exp. Ther., 2010, 335, 380-388.

[327] Pamplona, F.A.; Menezes-de-Lima, O.; Takahashi, R.N. Aspirintriggered lipoxin induces CB1-dependent catalepsy in mice. Neurosci. Lett., 2010, 470, 33-37. 
[328] Chicca, A.; Gachet, M.S.; Petrucci, V.; Schuehly, W.; Charles, R.P.; Gertsch, J. 4'-O-methylhonokiol increases levels of 2-arachidonoyl glycerol in mouse brain via selective inhibition of its COX-2mediated oxygenation. J. Neuroinflammation, 2015, 12, 89.

[329] Moody, J.S.; Kozak, K.R.; Ji, C.; Marnett, L.J. Selective oxygenation of the endocannabinoid 2-arachidonylglycerol by leukocyte-type 12lipoxygenase. Biochemistry, 2001, 40, 861-866.

[330] van Tienhoven, M.; Atkins, J.; Li, Y.; Glynn, P. Human neuropathy target esterase catalyzes hydrolysis of membrane lipids. J. Biol. Chem., 2002, 277, 20942-20948.

[331] Glaser, S.T.; Abumrad, N.A.; Fatade, F.; Kaczocha, M.; Studholme, K.M.; Deutsch, D.G. Evidence against the presence of an anandamide transporter. Proc. Natl. Acad. Sci. USA, 2003, 100(7), 4269-4274.

[332] Fowler, C.J. Transport of endocannabinoids across the plasma membrane and within the cell. FEBS J., 2013, 280(9), 1895-1904.

[333] Wu, Y.; Dissing-Olesen, L.; MacVicar, B.A.; Stevens, B. Microglia: Dynamic mediators of synapse development and plasticity. Trends Immunol., 2015, 36(10), 605-613.

[334] Gabrielli, M.; Battista, N.; Riganti, L.; Prada, I.; Antonucci, F.; Cantone, L.; Matteoli, M.; Maccarrone, M.; Verderio, C. Active endocannabinoids are secreted on extracellular membrane vesicles. EMBO Rep., 2015, 16(2), 213-220.

[335] Matsuda, L.A.; Bonner, T.I.; Lolait, S.J. Localization of cannabinoid receptor mRNA in rat brain. J. Comp. Neurol., 1993, 327, 535-550.

[336] Slipetz, D.M.; O'Neill, G.P.; Favreau, L.; Dufresne, C.; Gallant, M.; Gareau, Y.; Guay, D.; Labelle, M.; Metters, K.M. Activation of the human peripheral cannabinoid receptor results in inhibition of adenylyl cyclase. Mol. Pharmacol., 1995, 48(2), 352-361.

[337] Brown, S.M.; Wager-Miller, J.; Mackie, K. (2002) Cloning and molecular characterization of the rat $\mathrm{CB}_{2}$ cannabinoid receptor. Biochim. Biophys. Acta, 2002, 1576, 255-264.

[338] Galiègue, S.; Mary, S.; Marchand, J.; Dussossoy, D.; Carriere, D.; Carayon, P.; Bouaboula, M.; Shire, D.; Le Fur, G.; Casellas, P. Expression of central and peripheral cannabinoid receptors in human immune tissues and leukocyte subpopulations. Eur. J. Biochem., 1995, 232, 54-61.

[339] Irving, A.J.; McDonald, N.A.; Harkany, T. In: Cannabinoids and the Brain, Köfalvi, A., Ed. Springer US: 2008. pp. 59-73.

[340] Shire, D.; Carillon, C.; Kaghad, M.; Calandra, B.; Rinaldi-Carmona, M.; Le Fur, G.; Caput, D.; Ferrara, P. An amino-terminal variant of the central cannabinoid receptor resulting from alternative splicing. $J$. Biol. Chem., 1995, 270(8), 3726-3731.

[341] Ryberg, E.; Vu, H.K.; Larsson, N.; Groblewski, T.; Hjorth, S.; Elebring, T.; Sjögren, S.; Greasley, P.J. Identification and characterisation of a novel splice variant of the human $\mathrm{CB}_{1}$ receptor. FEBS Lett., 2005, 579(1), 259-264.

[342] Meschler, J.P.; Howlett, A.C. Signal transduction interactions between $\mathrm{CB}_{1}$ cannabinoid and dopamine receptors in the rat and monkey striatum. Neuropharmacology, 2001, 40, 918-926.

[343] Mackie, K.; Stella, N. Cannabinoid receptors and endocannabinoids: evidence for new players. AAPS J., 2006, 8, E298-E306.

[344] Zhang, H.Y.; Gao, M.; Liu, Q.R.; Bi, G.H.; Li, X.; Yang, H.J.; Gardner, E.L.; Wu, J.; Xi, Z.X. Cannabinoid $\mathrm{CB}_{2}$ receptors modulate midbrain dopamine neuronal activity and dopamine-related behavior in mice. Proc. Natl. Acad. Sci. USA, 2014, 111(46), E5007-5015.

[345] Zhang, H.Y.; Bi, G.H.; Li, X.; Li, J.; Qu, H.; Zhang, S.J.; Li, C.Y.; Onaivi, E.S.; Gardner, E.L.; Xi, Z.X.; Liu, Q.R. Species differences in cannabinoid receptor 2 and receptor responses to cocaine selfadministration in mice and rats. Neuropsychopharmacol., 2015, 40(4), 1037-1051.

[346] Howlett, A.C. Pharmacology of cannabinoid receptors. Ann. Rev. Pharmacol. Toxicol., 1995, 35, 607-634.

[347] Howlett, A.C.; Breivogel, C.S.; Childers, S.R.; Deadwyler, S.A.; Hampson, R.E.; Porrino, L.J. Cannabinoid physiology and pharmacology: 30 years of progress. Neuropharmacology, 2004, 47(S1), 345-358.

[348] Kaminski, N.E.; Koh, W.S.; Yang, K.H.; Lee, M.; Kessler, F.K. Suppression of the humoral immune response by cannabinoids is partially mediated through inhibition of adenylate cyclase by a pertussis toxin-sensitive $G$ protein coupled mechanism. Biochem. Pharmacol., 1994, 48, 1899-1908.

[349] Howlett, A.C.; Mukhopadhyay, S. Cellular signal transduction by anandamide and 2-arachidonoylglycerol. Chem. Phys. Lipids, 2000, 108, 53-70.

[350] Bouaboula, M.; Poinot-Chazel, C.; Marchand, J.; Canat, X.; Bourrie, B.; Rinaldi-Carmona, M.; Calandra, B.; Le Fur, G.; Casellas, P. Signaling pathway associated with stimulation of $\mathrm{CB}_{2}$ peripheral cannabinoid receptor. Involvement of both mitogen-activated protein kinase and induction of Krox-24 expression. Eur. J. Biochem., 1996, 237, 704-711.

[351] Bouaboula, M.; Perrachon, S.; Milligan, L.; Canat, X.; RinaldiCarmona, M.; Portier, M.; Barth, F.; Calandra, B.; Pecceu, F.; Lupker, J.; Maffrand, J.P.; Le Fur, G.; Casellas, P. A selective inverse agonist for central cannabinoid receptor inhibits mitogen-activated protein kinase activation stimulated by insulin or insulin-like growth factor 1. Evidence for a new model of receptor/ligand interactions. $J$. Biol. Chem., 1997, 272, 22330-22339.

[352] Sánchez, C.; Galve-Roperh, I.; Rueda, D.; Guzmán, M. Involvement of sphingomyelin hydrolysis and the mitogen-activated protein kinase cascade in the $\Delta^{9}$-tetrahydrocannabinol-induced stimulation of glucose metabolism in primary astrocytes. Mol. Pharmacol., 1998, 54, 834-843.

[353] Gómez del Pulgar, T.; Velasco, G.; Guzmán, M. The CB cannabinoid receptor is coupled to the activation of protein kinase B/Akt. Biochem. J., 2000, 347, 369-373.

[354] Rueda, D.; Galve-Roperh, I.; Haro, A.; Guzmán, M. The CB cannabinoid receptor is coupled to the action of c-Jun Nterminal kinase. Mol. Pharmacol., 2000, 58, 814-820.

[355] Galve-Roperh, I.; Rueda, D.; Gómez del Pulgar, T.; Velasco, G.; Guzmán, M. Mechanism of extracellular signal-regulated kinase activation by the $\mathrm{CB}_{1}$ cannabinoid receptor. Mol. Pharmacol., 2002, 62(6), 1385-1392.

[356] Molina-Holgado, E.; Vela, J.M.; Arevalo-Martin, A.; Almazan, G.; Molina-Holgado, F.; Borrell, J.; Guaza, C. Cannabinoids promote oligodendrocyte progenitor survival: involvement of cannabinoid receptors and phosphatidylinositol-3 kinase/Akt signaling. $J$. Neurosci., 2002, 22, 9742-9753.

[357] Molina-Holgado, F.; Pinteaux, E.; Heenan, L.; Moore, J.D.; Rothwell, N.J.; Gibson, R.M. Neuroprotective effects of the synthetic cannabinoid HU-210 in primary cortical neurons are mediated by phosphatidylinositol 3-kinase/Akt signaling. Mol. Cell. Neurosci., 2005, 28, 189-194.

[358] Viscomi, M.T.; Oddi, S.; Latini, L.; Pasquariello, N.; Florenzano, F.; Bernardi, G.; Molinari, M.; Maccarrone, M. Selective $\mathrm{CB}_{2}$ receptor agonism protects central neurons from remote axotomy-induced apoptosisthrough the PI3K/Akt pathway. J. Neurosci., 2009, 29, 4564-4570.

[359] Latini, L.; Bisicchia, E.; Sasso, V.; Chiurchiù, V.; Cavallucci, V.; Molinari, M.; Maccarrone, M.; Viscomi, M.T. Cannabinoid CB receptor $\left(\mathrm{CB}_{2} \mathrm{R}\right)$ stimulation delays rubrospinal mitochondrialdependent degeneration and improves functional recovery after spinal cord hemisection by ERK1/2 inactivation. Cell Death Dis., 2014, 5 , e1404.

[360] Guzmán, M.; Galve-Roperh, I.; Sánchez, C. Ceramide: a new second messenger of cannabinoid action. Trends Pharmacol. Sci., 2001, 22, 19-22.

[361] Harkany, T.; Guzmán, M.; Galve-Roperh, I.; Berghuis, P.; Devi, L.A.; Mackie, K. The emerging functions of endocannabinoid signaling during CNS development. Trends Pharmacol. Sci., 2007, 28(2), 83-92.

[362] Sánchez, C.; de Ceballos, M.L.; del Pulgar, T.G.; Rueda, D.; Corbacho, C.; Velasco, G.; Galve-Roperh, I.; Huffman, J.W.; Ramon y Cajal, S.; Guzmán, M. Inhibition of glioma growth in vivo by selective activation of the $\mathrm{CB}_{2}$ cannabinoid receptor. Cancer Res., 2001, 61, 5784-5789.

[363] Herrera, B.; Carracedo, A.; Diez-Zaera, M.; Gomez del Pulgar, T.; Guzman, M.; Velasco, G. The $\mathrm{CB}_{2}$ cannabinoid receptor signals apoptosis via ceramide-dependent activation of the mitochondrial intrinsic pathway. Exp. Cell. Res., 2006, 312, 2121-2131.

[364] Mackie, K.; Hille, B. Cannabinoids inhibit N-type calcium channels in neuroblastoma-glioma cells. PNAS, 1992, 89, 3825-3829.

[365] Felder, C.C.; Joyce, K.E.; Briley, E.M.; Mansouri, J.; Mackie, K.; Blond, O.; Lai, Y.; Ma, A.L.; Mitchell, R.L. Comparison of the pharmacology and signal transduction of the human cannabinoid $\mathrm{CB}_{1}$ and $\mathrm{CB}_{2}$ receptors. Mol. Pharm., 1995, 48, 443-450.

[366] Mackie, K.; Lai, Y.; Westenbroek, R.; Mitchell, R. Cannabinoids activate an inwardly rectifying potassium conductance and inhibit Qtype calcium currents in AtT20 cells transfected with rat brain cannabinoid receptor. J. Neurosci., 1995, 15, 6552-6561.

[367] McAllister, S.D.; Griffin, G.; Satin, L.S.; Abood, M.E. Cannabinoid receptors can activate and inhibit $G$ protein-coupled inwardly rectifying potassium channels in a xenopus oocyte expression system. J. Pharmacol. Exp. Ther., 1999, 291, 618-626.

[368] Roche, J.P.; Bounds, S.; Brown, S.; Mackie, K. A mutation in the second transmembrane region of the $\mathrm{CB}_{1}$ receptor selectively disrupts 
$\mathrm{G}$ protein signaling and prevents receptor internalization. Mol. Pharmacol., 1999, 56(3), 611-618.

[369] Lozovaya, N.; Min, R.; Tsintsadze, V.; Burnashev, N. Dual modulation of CNS voltage-gated calcium channels by cannabinoids: Focus on CB1 receptor-independent effects. Cell. Calcium., 2009, 46(3), 154-162.

[370] Calandra, B.; Portier, M.; Kernéis, A.; Delpech, M.; Carillon, C.; Le Fur, G.; Ferrara, P.; Shire, D. Dual intracellular signaling pathways mediated by the human cannabinoid $\mathrm{CB}_{1}$ receptor. Eur. J. Pharmacol., 1999, 374(3), 445-455.

[371] Lauckner, J.E.; Hille, B.; Mackie, K. The cannabinoid agonist WIN55,212-2 increases intracellular calcium via $\mathrm{CB}_{1}$ receptor coupling to $\mathrm{G}_{\mathrm{q} / 11} \mathrm{G}$ proteins. Proc. Natl. Acad. Sci. USA, 2005, 102, 19144-19149.

[372] Shoemaker, J.L.; Ruckle, M.B.; Mayeux, P.R.; Prather, P.L. Agonistdirected trafficking of response by endocannabinoids acting at $\mathrm{CB}_{2}$ receptors. J. Pharmacol. Exp. Ther., 2005, 315, 828-838.

[373] Ross, R.A.; Coutts, A.A.; McFarlane, S.M.; Anavi-Goffer, S.; Irving, A.J.; Pertwee, R.G.; MacEwan, D.J.; Scott, R.H. Actions of cannabinoid receptor ligands on rat cultured sensory neurones: implications for antinociception. Neuropharmacology, 2001, 40, 221232.

[374] Mukherjee, S.; Adams, M.; Whiteaker, K.; Daza, A.; Kage, K.; Cassar, S.; Meyer, M.; Yao, B.B. Species comparison and pharmacological characterization of rat and human $\mathrm{CB}_{2}$ cannabinoid receptors. Eur. J. Pharmacol., 2004, 505, 1-9.

[375] Bingham, B.; Jones, P.G.; Uveges, A.J.; Kotnis, S.; Lu, P.; Smith, V.A.; Sun, S.C.; Resnick, L.; Chlenov, M.; He, Y.; Strassle, B.W.; Cummons, T.A.; Piesla, M.J.; Harrison, J.E.; Whiteside, G.T.; Kennedy, J.D. Species-specific in vitro pharmacological effects of the cannabinoid receptor $2\left(\mathrm{CB}_{2}\right)$ selective ligand AM1241 and its resolved enantiomers. Br. J. Pharmacol., 2007, 151, 1061-1070.

[376] Atwood, B.K.; Wager-Miller, J.; Haskins, C.; Straiker, A.; Mackie, K. Functional selectivity in $\mathrm{CB}_{2}$ cannabinoid receptor signaling and regulation: implications for the therapeutic potential of $\mathrm{CB}_{2}$ ligands. Mol. Pharmacol., 2012, 81(2), 250-263.

[377] Haycock, D.A.; Kuster, J.E.; Stevenson, J.I.; Ward, S.J.; D'Ambra, T. Characterization of aminoalkylindole binding: selective displacement by cannabinoids. NIDA Res. Monogr., 1990, 105, 304-305.

[378] Bell, M.R.; D'Ambra, T.E.; Kumar, V.; Eissenstat, M.A.; Herrmann, J.L. Jr; Wetzel, J.R.; Rosi, D.; Philion, R.E.; Daum, S.J.; Hlasta, D.J.; Kullnig, R.K.; Ackerman, J.H.; Haubrich, D.R.; Luttinger, D.A.; Baizman, E.R.; Miller, M.S.; Ward S.J. Antinociceptive (aminoalkyl)indoles. J. Med. Chem., 1991, 34(3), 1099-1110.

[379] Martin, B.R.; Compton, D.R.; Thomas, B.F.; Prescott, W.R.; Little, P.J.; Razdan, R.K.; Johnson, M.R.; Melvin, L.S.; Mechoulam, R.; Ward, S.J. Behavioral, biochemical, and molecular modeling evaluations of cannabinoid analogs. Pharmacol. Biochem. Behav., 1991, 40(3), 471-478.

[380] Pertwee, R.G.; Howlett, A.C.; Abood, M.E.; Alexander, S.P.; Di Marzo, V.; Elphick, M.R.; Greasley, P.J.; Hansen, H.S.; Kunos, G.; Mackie, K.; Mechoulam, R.; Ross, R.A. International Union of Basic and Clinical Pharmacology. LXXIX. Cannabinoid receptors and their ligands: beyond $\mathrm{CB}_{1}$ and $\mathrm{CB}_{2}$. Pharmacol. Rev., 2010, 62(4), 588631.

[381] Pertwee, R.G. Receptors and channels targeted by synthetic cannabinoid receptor agonists and antagonists. Curr. Med. Chem., 2010, 17(14), 1360-1381.

[382] Clarke, W.P.; Bond, R.A. The elusive nature of intrinsic efficacy. Trends Pharmacol. Sci., 1998, 19(7), 270-276.

[383] Makita, N.; Iiri, T. Biased agonism: a novel paradigm in G proteincoupled receptor signaling observed in acquired hypocalciuric hypercalcemia. Endocr. J., 2014, 61(4), 303-309.

[384] Bonhaus, D.W.; Chang, L.K.; Kwan, J.; Martin, G.R. Dual activation and inhibition of adenylyl cyclase by cannabinoid receptor agonists: evidence for agonist-specific trafficking of intracellular responses. $J$. Pharmacol. Exp. Ther., 1998, 287(3), 884-888.

[385] Prather, P.L. In: Cannabinoids and the Brain; Köfalvi, A., Ed. Springer US, 2008, pp. 59-73.

[386] Mukhopadhyay, S.; Howlett, A.C. Chemically distinct ligands promote differential $\mathrm{CB}_{1}$ cannabinoid receptor-Gi protein interactions. Mol. Pharmacol., 2005, 67(6), 2016-2024.

[387] Howlett, A.C.; Fleming, R.M. Cannabinoid inhibition of adenylatecyclase-pharmacology of the response in neuroblastoma cell membranes. Mol. Pharmacol., 1984, 26, 532-538.

[388] Griffin, G.; Atkinson, P.J.; Showalter, V.M.; Martin, B.R.; Abood, M.E. Evaluation of cannabinoid receptor agonists and antagonists using the guanosine-5'-O-(3-[ $\left.{ }^{35} \mathrm{~S}\right]$ thio)-triphosphate binding assay in rat cerebellar membranes. J. Pharmacol. Exp. Ther., 1998, 285(2), 553-560.

[389] Cinar, R.; Szücs, M. CB 1 receptor-independent actions of SR141716 on $\mathrm{G}$-protein signaling: coapplication with the $\mu$-opioid agonist TyrD-Ala-Gly-(NMe)Phe-Gly-ol unmasks novel, pertussis toxininsensitive opioid signaling in $\mu$-opioid receptor-Chinese hamster ovary cells. J. Pharmacol. Exp. Ther., 2009, 330(2), 567-574.

[390] Köfalvi, A.; Rodrigues, R.J.; Ledent, C.; Mackie, K.; Vizi, E.S.; Cunha, R.A.; Sperlágh, B. Involvement of cannabinoid receptors in the regulation of neurotransmitter release in the rodent striatum: a combined immunochemical and pharmacological analysis. $J$. Neurosci., 2005, 25, 2874-2884.

[391] Köfalvi, A.; Pereira, M.F.; Rebola, N.; Rodrigues, R.J.; Oliveira, C.R.; Cunha, R.A. Anandamide and NADA bi-directionally modulate presynaptic $\mathrm{Ca}^{2+}$ levels and transmitter release in the hippocampus. Br. J. Pharmacol., 2007, 151(4), 551-563.

[392] Pertwee, R.G. Inverse agonism and neutral antagonism at cannabinoid $\mathrm{CB}_{1}$ receptors. Life Sci., 2005, 76(12), 1307-1324.

[393] Wiley, J.L.; Breivogel, C.S.; Mahadevan, A.; Pertwee, R.G.; Cascio, M.G.; Bolognini, D.; Huffman, J.W.; Walentiny, D.M.; Vann, R.E.; Razdan, R.K.; Martin, B.R. Structural and pharmacological analysis of O-2050, a putative neutral cannabinoid $\mathrm{CB}_{1}$ receptor antagonist. Eur. J .Pharmacol., 2011, 651(1-3), 96-105.

[394] Ruiu, S.; Pinna, G.A.; Marchese, G.; Mussinu, J.M.; Saba, P.; Tambaro, S.; Casti, P.; Vargiu, R.; Pani, L. Synthesis and characterization of NESS 0327: a novel putative antagonist of the $\mathrm{CB}_{1}$ cannabinoid receptor. J. Pharmacol. Exp. Ther., 2003, 306(1), 363-370.

[395] Meye, F.J.; Trezza, V.; Vanderschuren, L.J.; Ramakers, G.M.; Adan, R.A. Neutral antagonism at the cannabinoid 1 receptor: a safer treatment for obesity. Mol. Psychiatry, 2013, 18(12), 1294-1301.

[396] Ferreira, S.G.; Teixeira, F.M.; Garção, P.; Agostinho, P.; Ledent, C.; Cortes, L.; Mackie, K.; Köfalvi, A. Presynaptic $\mathrm{CB}_{1}$ cannabinoid receptors control frontocortical serotonin and glutamate release species differences. Neurochem. Int., 2012, 61(2), 219-226.

[397] Bitencourt, R.M.; Alpár, A.; Cinquina, V.; Ferreira, S.G.; Pinheiro, B.S.; Lemos, C.; Ledent, C.; Takahashi, R.N.; Sialana, F.J.; Lubec, G.; Cunha, R.A.; Harkany, T.; Köfalvi, A. Lack of presynaptic interaction between glucocorticoid and $\mathrm{CB}_{1}$ cannabinoid receptors in GABA- and glutamatergic terminals in the frontal cortex of laboratory rodents. Neurochem. Int., 2015, 90, 72-84.

[398] Wade, M.R.; Tzavara, E.T.; Nomikos, G.G. Cannabinoids reduce cAMP levels in the striatum of freely moving rats: an in vivo microdialysis study. Brain Res., 2004, 1005(1-2), 117-123.

[399] Felder, C.C.; Joyce, K.E.; Briley, E.M.; Glass, M.; Mackie, K.P.; Fahey, K.J.; Cullinan, G.J.; Hunden, D.C.; Johnson, D.W.; Chaney, M.O.; Koppel, G.A.; Brownstein, M. LY320135, a novel cannabinoid $\mathrm{CB}_{1}$ receptor antagonist, unmasks coupling of the $\mathrm{CB}_{1}$ receptor to stimulation of cAMP accumulation. J. Pharmacol. Exp. Ther., 1998, 284, 291-297.

[400] Hermans, E. Biochemical and pharmacological control of the multiplicity of coupling at G-protein-coupled receptors. Pharmacol. Ther., 2003, 99(1), 25-44.

[401] Straiker, A.; Mitjavila, J.; Yin, D.; Gibson, A.; Mackie, K. Aiming for allosterism: Evaluation of allosteric modulators of $\mathrm{CB}_{1}$ in a neuronal model. Pharmacol. Res., 2015, 99, 370-376.

[402] Price, M.R.; Baillie, G.L.; Thomas, A.; Stevenson, L.A.; Easson, M Goodwin, R.; McLean, A.; McIntosh, L.; Goodwin, G.; Walker, G.; Westwood, P.; Marrs, J.; Thomson, F.; Cowley, P.; Christopoulos, A.; Pertwee, R.G.; Ross, R.A. Allosteric modulation of the cannabinoid CB1 receptor. Mol. Pharmacol., 2005, 68(5), 1484-1495.

[403] Horswill, J.G.; Bali, U.; Shaaban, S.; Keily, J.F.; Jeevaratnam, P.; Babbs, A.J.; Reynet, C.; Wong Kai In, P. PSNCBAM-1, a novel allosteric antagonist at cannabinoid CB1 receptors with hypophagic effects in rats. Br. J. Pharmacol., 2007, 152, 805-814.

[404] Oddi, S.; Dainese, E.; Fezza, F.; Lanuti, M.; Barcaroli, D.; De Laurenzi, V.; Centonze, D.; Maccarrone, M. Functional characterization of putative cholesterol binding sequence (CRAC) in human type-1 cannabinoid receptor: CRAC sequence in human cannabinoid receptors. J. Neurochem., 2011, 116, 858-865.

[405] Stornaiuolo, M.; Bruno, A.; Botta, L.; La Regina, G.; Cosconati, S.; Silvestri, R.; Marinelli, L.; Novellino, E. Endogenous vs exogenous allosteric modulators in GPCRs: A dispute for shuttling CB1 among different membrane microenvironments. Sci. Rep., 2015, 5, 15453.

[406] Vallée, M.; Vitiello, S.; Bellocchio, L.; Hébert-Chatelain, E.; Monlezun, S.; Martin-Garcia, E.; Kasanetz, F.; Baillie, G.L.; Panin, F.; Cathala, A.; Roullot-Lacarrière, V.; Fabre, S.; Hurst, D.P.; Lynch, D.L.; Shore, D.M.; Deroche-Gamonet, V.; Spampinato, U.; Revest, 
J.M.; Maldonado, R.; Reggio, P.H.; Ross, R.A.; Marsicano, G.; Piazza, P.V. Pregnenolone can protect the brain from cannabis intoxication. Science, 2014, 343(6166), 94-98.

[407] Navarro, H.A.; Howard, J.L.; Pollard, G.T.; Carroll, F.I. Positive allosteric modulation of the human cannabinoid (CB) receptor by RTI-371, a selective inhibitor of the dopamine transporter. Br. J. Pharmacol., 2009, 156(7), 1178-1184.

[408] Pamplona, F.A.; Ferreira, J.; Menezes de Lima, O. Jr.; Duarte, F.S.; Bento, A.F.; Forner, S.; Villarinho, J.G.; Bellocchio, L.; Wotjak, C.T.; Lerner, R.; Monory, K.; Lutz, B.; Canetti, C.; Matias, I.; Calixto, J.B.; Marsicano, G.; Guimarães, M.Z.; Takahashi, R.N. Antiinflammatory lipoxin A4 is an endogenous allosteric enhancer of CB1 cannabinoid receptor. Proc. Natl. Acad. Sci. USA, 2012, 109, 21134-21139.

[409] Mechoulam, R.; Lander, N.; Srebnik, M.; Breuer, A.; Segal, M.; Feigenbaum, J.J.; Jarbe, T.U.; Consroe, P. Stereochemical requirements for cannabimimetic activity. NIDA Res. Monogr., 1987, 79, 15-30.

[410] Petitet, F.; Jeantaud, B.; Reibaud, M.; Imperato, A.; Dubroeucq, M.C. Complex pharmacology of natural cannabinoids: evidence for partial agonist activity of $\Delta^{9}$-tetrahydrocannabinol and antagonist activity of cannabidiol on rat brain cannabinoid receptors. Life Sci., 1998, 63(1), PL1-6.

[411] McPartland, J.M.; Glass, M.; Pertwee, R.G. Meta-analysis of cannabinoid ligand binding affinity and receptor distribution: interspecies differences. Br. J .Pharmacol., 2007, 152(5), 583-593.

[412] Ryberg, E.; Larsson, N.; Sjögren, S.; Hjorth, S.; Hermansson, N.O.; Leonova, J.; Elebring, T.; Nilsson, K.; Drmota, T.; Greasley, P.J. The orphan receptor GPR55 is a novel cannabinoid receptor. Br. J. Pharmacol., 2007, 152(7), 1092-1101.

[413] Harris, R.A.; Stokes, J.A. Cannabinoids inhibit calcium uptake by brain synaptosomes. J. Neurosci., 1982, 2(4), 443-447.

[414] Wood, T.B.; Spivey, W.T.N.; Easterfield, T.H. Charas, the resin of Indian hemp. J. Chem. Soc., 1986, 69, 539-546.

[415] Pertwee, R.G. The diverse $\mathrm{CB}_{1}$ and $\mathrm{CB}_{2}$ receptor pharmacology of three plant cannabinoids: $\Delta^{9}$-tetrahydrocannabinol, cannabidiol and $\Delta^{9}$-tetrahydrocannabivarin. Br. J. Pharmacol., 2008, 153, 199-215.

[416] Gill, E.W.; Paton, W.D.; Pertwee, R.G. Preliminary experimentson the chemistry and pharmacology of cannabis. Nature, 1970, 228, 134136.

[417] Pertwee, R.G.; Thomas, A.; Stevenson, L.A.; Ross, R.A.; Varvel, S.A.; Lichtman, A.H.; Martin, B.R.; Razdan, R.K. The psychoactive plant cannabinoid, Delta ${ }^{9}$-tetrahydrocannabinol, is antagonized by Delta $^{8}$ - and Delta ${ }^{9}$-tetrahydrocannabivarin in mice in vivo. Br. $J$. Pharmacol., 2007, 150(5), 586-594.

[418] Fernández-Ruiz, J.; Sagredo, O.; Pazos, M.R.; García, C.; Pertwee, R.; Mechoulam, R.; Martínez-Orgado, J. Cannabidiol for neurodegenerative disorders: important new clinical applications for this phytocannabinoid? Br. J. Clin. Pharmacol., 2013, 75(2), 323333.

[419] Stanley, C.P.; Hind, W.H.; Tufarelli, C.; O'Sullivan, S.E. Cannabidiol causes endothelium-dependent vasorelaxation of human mesenteric arteries via $\mathrm{CB}_{1}$ activation. Cardiovasc. Res., 2015, 107(4), 568-578.

[420] Thomas, A.; Baillie, G.L.; Phillips, A.M.; Razdan, R.K.; Ross, R.A.; Pertwee, R.G. Cannabidiol displays unexpectedly high potency as an antagonist of $\mathrm{CB}_{1}$ and $\mathrm{CB}_{2}$ receptor agonists in vitro. Br. J. Pharmacol., 2007, 150(5), 613-623.

[421] Laprairie, R.B.; Bagher, A.M.; Kelly, M.E.; Denovan-Wright, E.M. Cannabidiol is a negative allosteric modulator of the type 1 cannabinoid receptor. Br. J .Pharmacol., 2015, 172(20), 4790-4805.

[422] Gertsch, J.; Leonti, M.; Raduner, S.; Racz, I.; Chen, J.Z.; Xie, X.Q.; Altmann, K.H.; Karsak, M.; Zimmer, A. $\beta$-caryophyllene is a dietary cannabinoid. Proc. Natl. Acad. Sci. USA, 2008, 105(26), 9099-9104.

[423] Bahi, A.; Al Mansouri, S.; Al Memari, E.; Al Ameri, M.; Nurulain, S.M.; Ojha, S. $\beta$-Caryophyllene, a $\mathrm{CB}_{2}$ receptor agonist produces multiple behavioral changes relevant to anxiety and depression in mice. Physiol. Behav., 2014, 135, 119-124.

[424] Davis, W.M.; Hatoum, N.S. Neurobehavioral actions of cannabichromene and interactions with delta 9-tetrahydrocannabinol. Gen. Pharmacol., 1983, 14(2), 247-252.

[425] Maione, S.; Piscitelli, F.; Gatta, L.; Vita, D.; De Petrocellis, L.; Palazzo, E.; de Novellis, V.; Di Marzo, V. Non-psychoactive cannabinoids modulate the descending pathway of antinociception in anaesthetized rats through several mechanisms of action. Br. J. Pharmacol., 2011, 162(3), 584-596.

[426] Hanuš, L.; Abu-Lafi, S.; Fride, E.; Breuer, A.; Vogel, Z.; Shalev, D.E.; Kustanovich, I.; Mechoulam, R. 2-Arachidonyl glyceryl ether, an endogenous agonist of the cannabinoid CB1 receptor. Proc. Natl. Acad. Sci. USA, 2001, 98, 3662-3665.

[427] Bisogno, T.; Melck, D.; Bobrov, M.Yu.; Gretskaya, N.M.; Bezuglov, V.V.; De Petrocellis, L.; Di Marzo, V. N-acyl-dopamines: novel synthetic $\mathrm{CB}_{1}$ cannabinoid-receptor ligands and inhibitors of anandamide inactivation with cannabimimetic activity in vitro and in vivo. Biochem. J., 2000, 351(3), 817-824.

[428] Leggett, J.D.; Aspley, S.; Beckett, S.R.; D'Antona, A.M.; Kendall, D.A.; Kendall, D.A. Oleamide is a selective endogenous agonist of rat and human CB1 cannabinoid receptors. Br. J .Pharmacol., 2004 141(2), 253-262.

[429] Barg, J.; Fride, E.; Hanuš, L.; Levy, R.; Matus-Leibovitch, N.; Heldman, E.; Bayewitch, M.; Mechoulam, R.; Vogel, Z. Cannabinomimetic behavioral effects of and adenylate cyclase inhibition by two new endogenous anandamides. Eur. J. Pharmacol., 1995, 287(2), 145-152.

[430] Walter, L.A.; Franklin, A.; Mhyre, A.; Mackie, K.; Stella, N. Microglial cells produce endocannabinoids and express functional cannabinoid receptors Evidence that docosatetraenylethanolamide is an additional endocannabinoid. Society For Neuroscience Abstracts, 2001, 27(2), 1671.

[431] Walter, L.; Franklin, A.; Witting, A.; Moller, T.; Stella, N. Astrocytes in culture produce anandamide and other acylethanolamides. J. Biol. Chem., 2002, 277(23), 20869-20876.

[432] Porter, A.C.; Sauer, J.M.; Knierman, M.D.; Becker, G.W.; Berna, M.J.; Bao, J.; Nomikos, G.G.; Carter, P.; Bymaster, F.P.; Leese, A.B.; Felder, C.C. Characterization of a novel endocannabinoid, virodhamine, with antagonist activity at the $\mathrm{CB}_{1}$ receptor. $J$. Pharmacol. Exp. Ther., 2002, 301(3), 1020-1024.

[433] Abadji, V.; Lin, S.; Taha, G.; Griffin, G.; Stevenson, L.A.; Pertwee, R.G.; Makriyannis, A. (R)-methanandamide: a chiral novel anandamide possessing higher potency and metabolic stability. $J$. Med. Chem., 1994, 37(12), 1889-1893.

[434] Hillard, C.J.; Manna, S.; Greenberg, M.J.; DiCamelli, R.; Ross, R.A.; Stevenson, L.A.; Murphy, V.; Pertwee, R.G.; Campbell, W.B. Synthesis and characterization of potent and selective agonists of the neuronal cannabinoid receptor $\left(\mathrm{CB}_{1}\right)$. J. Pharmacol. Exp. Ther., 1999, 289(3), 1427-1433

[435] Gatley, S.J.; Lan, R.; Pyatt, B.; Gifford, A.N.; Volkow, N.D.; Makriyannis, A. Binding of the non-classical cannabinoid CP 55,940, and the diarylpyrazole AM251 to rodent brain cannabinoid receptors. Life Sci., 1997, 61(14), PL 191-197.

[436] Lan, R.; Gatley, J.; Lu, Q.; Fan, P.; Fernando, S.R.; Volkow, N.D.; Pertwee, R.; Makriyannis, A. Design and synthesis of the $\mathrm{CB}_{1}$ selective cannabinoid antagonist AM281: a potential human SPECT ligand. AAPS PharmSci., 1999, 1(2), E4.

[437] Huffman, J.W.; Liddle, J.; Yu, S.; Aung, M.M.; Abood, M.E.; Wiley, J.L.; Martin, B.R. 3-(1',1'-Dimethylbutyl)-1-deoxy-delta8-THC and related compounds: synthesis of selective ligands for the CB2 receptor. Bioorg. Med. Chem., 1999, 7, 2905-2914.

[438] Hanuš, L.; Breuer, A.; Tchilibon, S.; Shiloah, S.; Goldenberg, D.; Horowitz, M.; Pertwee, R.G.; Ross, R.A.; Mechoulam, R.; Fride, E. HU-308: a specific agonist for $\mathrm{CB}_{2}$, a peripheral cannabinoid receptor. Proc. Natl. Acad. Sci. USA, 1999, 96(25), 14228-14233.

[439] Murineddu, G.; Lazzari, P.; Ruiu, S.; Sanna, A.; Loriga, G.; Manca, I.; Falzoi, M.; Dessì, C.; Curzu, M.M.; Chelucci, G.; Pani, L.; Pinna, G.A. Tricyclic pyrazoles. 4. Synthesis and biological evaluation of analogues of the robust and selective CB2 cannabinoid ligand 1(2',4'-dichlorophenyl)-6-methyl-N-piperidin-1-yl-1,4dihydroindeno[1,2-c]pyrazole-3-carboxamide. J. Med. Chem., 2006, 49, 7502-7512.

[440] Hosohata, K.; Quock, R.M.; Hosohata, Y.; Burkey, T.H.; Makriyannis, A.; Consroe, P.; Roeske, W.R.; Yamamura, H.I AM630 is a competitive cannabinoid receptor antagonist in the guinea pig brain. Life Sci., 1997, 61(9), PL115-118.

[441] Rinaldi-Carmona, M.; Barth, F.; Millan, J.; Derocq, J.M.; Casellas, P.; Congy, C.; Oustric, D.; Sarran, M.; Bouaboula, M.; Calandra, B.; Portier, M.; Shire, D.; Brelière, J.C.; Le Fur, G.L. SR144528, the first potent and selective antagonist of the $\mathrm{CB}_{2}$ cannabinoid receptor. $J$. Pharmacol. Exp. Ther., 1998, 284, 644-650.

[442] Iwamura, H.; Suzuki, H.; Ueda, Y.; Kaya, T.; Inaba, T. In vitro and in vivo pharmacological characterization of JTE-907, a novel selective ligand for cannabinoid $\mathrm{CB}_{2}$ receptor. J. Pharmacol. Exp. Ther., 2001, 296(2), 420-425.

[443] Savinainen, J.R.; Kokkola, T.; Salo, O.M.; Poso, A.; Järvinen, T. Laitinen, J.T. Identification of WIN55212-3 as a competitive neutral antagonist of the human cannabinoid CB2 receptor. Br. J .Pharmacol., 2005, 145(5), 636-645. 
[444] Smith, T.H.; Sim-Selley, L.J.; Selley, D.E. Cannabinoid CB1 receptor-interacting proteins: novel targets for central nervous system drug discovery? Br. J .Pharmacol., 2010, 160(3), 454-466.

[445] Gainetdinov, R.R.; Premont, R.T.; Bohn, L.M.; Lefkowitz, R.J.; Caron, M.G. Desensitization of $G$ protein-coupled receptors and neuronal functions. Annu. Rev. Neurosci., 2004, 27, 107-144.

[446] Gurevich, E.V.; Tesmer, J.J.; Mushegian, A.; Gurevich, V.V. G protein-coupled receptor kinases: more than just kinases and not only for GPCRs. Pharmacol. Ther., 2012, 133, 40-69.

[447] Jin, W.; Brown, S.; Roche, J.P.; Hsieh, C.; Celver, J.P.; Kovoor, A.; Chavkin, C.; Mackie, K. Distinct domains of the CB1 cannabinoid receptor mediate desensitization and internalization. J. Neurosci., 1999, 19(10), 3773-3780.

[448] Gyombolai, P.; Boros, E.; Hunyady, L.; Turu, G. Differential $\beta$ arrestin2 requirements for constitutive and agonist-induced internalization of the $\mathrm{CB}_{1}$ cannabinoid receptor. Mol. Cell. Endocrinol., 2013, 372(1-2), 116-127.

[449] Daigle, T.L.; Kearn, C.S.; Mackie, K. Rapid $\mathrm{CB}_{1}$ cannabinoid receptor desensitization defines the time course of ERK1/2 MAP kinase signaling. Neuropharmacology, 2008, 54(1), 36-44.

[450] Rubino, T.; Viganò, D.; Premoli, F.; Castiglioni, C.; Bianchessi, S.; Zippel, R.; Parolaro, D. Changes in the expression of $G$ proteincoupled receptor kinases and beta-arrestins in mouse brain during cannabinoid tolerance: a role for RAS-ERK cascade. Mol. Neurobiol., 2006, 33, 199-213.

[451] Sim-Selley, L.J.; Schechter, N.S.; Rorrer, W.K.; Dalton, G.D.; Hernandez, J.; Martin, B.R.; Selley, D.E. Prolonged recovery rate of $\mathrm{CB}_{1}$ receptor adaptation after cessation of long-term cannabinoid administration. Mol. Pharmacol., 2006, 70, 986-996.

[452] Villares, J. Chronic use of marijuana decreases cannabinoid receptor binding and mRNA expression in the human brain. Neuroscience, 2007, 145, 323-334.

[453] Breivogel, C.S.; Puri, V.; Lambert, J.M.; Hill, D.K.; Huffman, J.W.; Razdan, R.K. The influence of beta-arrestin2 on cannabinoid $\mathrm{CB}_{1}$ receptor coupling to G-proteins and subcellular localization and relative levels of beta-arrestin 1 and 2 in mouse brain. J. Recept Signal Transduct. Res., 2013, 33(6), 67-79.

[454] Luk, T.; Jin, W.; Zvonok, A.; Lu, D.; Lin, X.Z.; Chavkin, C.; Makriyannis, A.; Mackie, K. Identification of a potent and highly efficacious, yet slowly desensitizing $\mathrm{CB}_{1}$ cannabinoid receptor agonist. Br. J. Pharmacol., 2004, 142(3), 495-500.

[455] Ahn, K.H.; Mahmoud, M.M.; Shim, J.Y.; Kendall, D.A. Distinct roles of $\beta$-arrestin 1 and $\beta$-arrestin 2 in ORG27569-induced biased signaling and internalization of the cannabinoid receptor 1 (CB1). $J$. Biol. Chem., 2013, 288(14), 9790-9800.

[456] Hájková, A.; Techlovská, Š.; Dvořáková, M.; Chambers, J.N.; Kumpošt, J.; Hubálková, P.; Prezeau, L.; Blahos, J. SGIP1 alters internalization and modulates signaling of activated cannabinoid receptor 1 in a biased manner. Neuropharmacology, 2016, 107, 201214.

[457] Grimsey, N.L.; Graham, E.S.; Dragunow, M.; Glass, M. Cannabinoid Receptor 1 trafficking and the role of the intracellular pool: implications for therapeutics. Biochem. Pharmacol., 2010, 80, 10501062.

[458] Duarte, J.M.N.; Ferreira, S.G.; Carvalho, R.A.; Cunha, R.A.; Köfalvi, A. $\mathrm{CB}_{1}$ receptor activation inhibits neuronal and astrocytic intermediary metabolism in the rat hippocampus. Neurochem. Int., 2012, 60, 1-8.

[459] Hebert-Chatelain, E.; Reguero, L.; Puente, N.; Lutz, B.; Chaouloff, F.; Rossignol, R.; Piazza, P.V.; Benard, G.; Grandes, P.; Marsicano, G. Cannabinoid control of brain bioenergetics: Exploring the subcellular localization of the CB1 receptor. Mol. Metab., 2014, 3(4), 495-504.

[460] Martini, L.; Waldhoer, M.; Pusch, M.; Kharazia, V.; Fong, J.; Lee, J.H.; Freissmuth, C.; Whistler, J.L. Ligand-induced down-regulation of the cannabinoid 1 receptor is mediated by the G-protein-coupled receptor-associated sorting protein GASP1. FASEB J., 2007, 21, 802811.

[461] Tappe-Theodor, A.; Agarwal, N.; Katona, I.; Rubino, T.; Martini, L.; Swiercz, J.; Mackie, K.; Monyer, H.; Parolaro, D.; Whistler, J.; Kuner, T.; Kuner, R. A molecular basis of analgesic tolerance to cannabinoids. J. Neurosci., 2007, 27(15), 4165-4177.

[462] Botanas, C.J.; de la Peña, J.B.; Dela Pena, I.J.; Tampus, R.; Kim, H.J.; Yoon, S.S.; Seo, J.W.; Jeong, E.J.; Cheong, J.H. Evaluation of the abuse potential of AM281, a new synthetic cannabinoid $\mathrm{CB}_{1}$ receptor antagonist. Eur. J. Pharmacol., 2015, 766, 135-141.
[463] Rozenfeld, R.; Devi, L.A. Regulation of $\mathrm{CB}_{1}$ cannabinoid receptor trafficking by the adaptor protein AP-3. FASEB J., 2008, 22(7), 23112322.

[464] Niehaus, J.L.; Liu, Y.; Wallis, K.T.; Egertová, M.; Bhartur, S.G.; Mukhopadhyay, S.; Shi, S.; He, H.; Selley, D.E.; Howlett, A.C.; Elphick, M.R.; Lewis, D.L. CB1 cannabinoid receptor activity is modulated by the cannabinoid receptor interacting protein CRIP $1 \mathrm{a}$. Mol. Pharmacol., 2007, 72(6), 1557-1566.

[465] Smith, T.H.; Blume, L.C.; Straiker, A.; Cox, J.O.; David, B.G.; McVoy, J.R.; Sayers, K.W.; Poklis, J.L.; Abdullah, R.A.; Egertová, M.; Chen, C.K.; Mackie, K.; Elphick, M.R.; Howlett, A.C.; Selley, D.E. Cannabinoid receptor-interacting protein 1a modulates $\mathrm{CB}_{1}$ receptor signaling and regulation. Mol. Pharmacol., 2015, 87(4), 747765.

[466] Guggenhuber, S.; Alpár, A.; Chen, R.; Schmitz, N.; Wickert, M.; Mattheus, T.; Harasta, A.E.; Purrio, M.; Kaiser, N.; Elphick, M.R.; Monory, K.; Kilb, W.; Luhmann, H.J.; Harkany, T.; Lutz, B.; Klugmann, M. Cannabinoid receptor-interacting protein Crip1a modulates $\mathrm{CB}_{1}$ receptor signaling in mouse hippocampus. Brain Struct. Funct., 2016, 221(4), 2061-2074.

[467] Blume, L.C.; Eldeeb, K.; Bass, C.E.; Selley, D.E.; Howlett, A.C. Cannabinoid receptor interacting protein (CRIP1a) attenuates $\mathrm{CB}_{1} \mathrm{R}$ signaling in neuronal cells. Cell. Signal., 2015, 27(3), 716-726.

[468] Stauffer, B.; Wallis, K.T.; Wilson, S.P.; Egertová, M.; Elphick, M.R.; Lewis, D.L.; Hardy, L.R. CRIP1a switches cannabinoid receptor agonist/antagonist-mediated protection from glutamate excitotoxicity. Neurosci. Lett., 2011, 503(3), 224-228.

[469] Ludányi, A.; Erőss, L.; Czirják, S.; Vajda, J.; Halász, P.; Watanabe, M.; Palkovits, M.; Maglóczky, Z.; Freund, T.F.; Katona, I Downregulation of the $\mathrm{CB}_{1}$ cannabinoid receptor and related molecular elements of the endocannabinoid system in epileptic human hippocampus. J. Neurosci., 2008, 28(12), 2976-2990.

[470] McGuinness, D.; Malikzay, A.; Visconti, R.; Lin, K.; Bayne, M.; Monsma, F.; Lunn, C.A. Characterizing cannabinoid $\mathrm{CB}_{2}$ receptor ligands using DiscoveRx PathHunter beta-arrestin assay. J. Biomol. Screen., 2009, 14(1), 49-58.

[471] Mukhopadhyay, P.; Baggelaar, M.; Erdelyi, K.; Cao, Z.; Cinar, R.; Fezza, F.; Ignatowska-Jankowska, B.; Wilkerson, J.; van Gils, N.; Hansen, T.; Ruben, M.; Soethoudt, M.; Heitman, L.; Kunos, G.; Maccarrone, M.; Lichtman, A.; Pacher, P.; van der Stelt, M. The novel, orally available and peripherally restricted selective cannabinoid $\mathrm{CB}_{2}$ receptor agonist LEI-101 prevents cisplatin-induced nephrotoxicity. Br. J .Pharmacol., 2016, 173(3), 446-458.

[472] Gantz, I.; Muraoka, A.; Yang, Y.K.; Samuelson, L.C.; Zimmerman, E.M.; Cook, H.; Yamada, T. Cloning and chromosomal localization of a gene (GPR18) encoding a novel seven transmembrane receptor highly expressed in spleen and testis. Genomics, 1997, 42(3), 462466.

[473] McHugh, D.; Page, J.; Dunn, E.; Bradshaw, H.B. $\Delta^{9}-$ Tetrahydrocannabinol and $\mathrm{N}$-arachidonyl glycine are full agonists at GPR18 receptors and induce migration in human endometrial HEC1B cells. Br. J .Pharmacol., 2012, 165(8), 2414-2424.

[474] Járai, Z.; Wagner, J.A.; Varga, K.; Lake, K.D.; Compton, D.R.; Martin, B.R.; Zimmer, A.M.; Bonner, T.I.; Buckley, N.E.; Mezey, E.; Razdan, R.K.; Zimmer, A.; Kunos, G. Cannabinoid-induced mesenteric vasodilation through an endothelial site distinct from CB1 or CB2 receptors. Proc. Natl. Acad. Sci. USA, 1999, 96(24), 1413614141.

[475] Mo, F.M.; Offertáler, L.; Kunos, G. Atypical cannabinoid stimulates endothelial cell migration via a $G_{i} / G_{0}$-coupled receptor distinct from CB1, CB2 or EDG-1. Eur. J .Pharmacol., 2004, 489(1-2), 21-27.

[476] Kohno, M.; Hasegawa, H.; Inoue, A.; Muraoka, M.; Miyazaki, T.; Ok,a K.; Yasukawa, M. Identification of N-arachidonylglycine as the endogenous ligand for orphan G-protein-coupled receptor GPR18. Biochem. Biophys. Res. Commun., 2006, 347(3), 827-832.

[477] McHugh, D.; Hu, S.S.; Rimmerman, N.; Juknat, A.; Vogel, Z.; Walker, J.M.; Bradshaw, H.B. N-arachidonoyl glycine, an abundant endogenous lipid, potently drives directed cellular migration through GPR18, the putative abnormal cannabidiol receptor. BMC Neuroscience, 2010, 11, 44.

[478] McHugh, D.; Bradshaw, H.B. In: Endocannabinoids: actions at nonCB1/CB2 cannabinoid receptors, Abood, M.E., Ed. Springer: New York, 2012. pp. 135-142

[479] McHugh, D.; Roskowski, D.; Xie, S.; Bradshaw, H.B. $\Delta^{9}$-THC and $\mathrm{N}$-arachidonoyl glycine regulate BV-2 microglial morphology and cytokine release plasticity: implications for signaling at GPR18. Front. Pharmacol., 2014, 4, 162 . 
[480] Chiang, N.; Dalli, J.; Colas, R.A.; Serhan, C.N. Identification of resolvin D2 receptor mediating resolution of infections and organ protection. J. Exp. Med., 2015, 212(8), 1203-1217.

[481] Kakarala, K.K.; Jamil, K. Sequence-structure based phylogeny of GPCR Class A Rhodopsin receptors. Mol. Phylogenet. Evol., 2014, 74, 66-96.

[482] Wagner, J.A.; Varga, K.; Jarai, Z.; Kunos, G. Mesenteric vasodilation mediated by endothelial anandamide receptors. Hypertension, 1999, 33, 429-434.

[483] Alexander, S.P. So what do we call GPR18 now? Br. J. Pharmacol, 2012, 165(8), 2411-2413.

[484] Caldwell, M.D.; Hu, S.S.; Viswanathan, S.; Bradshaw, H.; Kelly, M.E.; Straiker, A. A GPR18-based signalling system regulates IOP in murine eye. Br. J. Pharmacol., 2013, 169(4), 834-843.

[485] Console-Bram, L.; Brailoiu, E.; Brailoiu, G.C.; Sharir, H.; Abood, M.E. Activation of GPR18 by cannabinoid compounds: a tale of biased agonism. Br. J. Pharmacol., 2014, 171(16), 3908-3917.

[486] Lu, V.B.; Puhl, H.L.; Ikeda, S.R. N-Arachidonyl glycine does not activate $G$ protein-coupled receptor 18 signaling via canonical pathways. Mol. Pharm., 2013, 83(1), 267-282.

[487] Offertáler, L.; Mo, F.M.; Bátkai, S.; Liu, J.; Begg, M.; Razdan, R.K.; Martin, B.R.; Bukoski, R.D.; Kunos, G. Selective ligands and cellular effectors of a $\mathrm{G}$ protein-coupled endothelial cannabinoid receptor. Mol. Pharmacol., 2003, 63(3), 699-705.

[488] Sawzdargo, M.; Nguyen, T.; Lee, D.K.; Lynch, K.R.; Cheng, R.; Heng, H.H.; George, S.R.; O'Dowd, B.F. Identification and cloning of three novel human $G$ protein-coupled receptor genes GPR52, PsiGPR53 and GPR55: GPR55 is extensively expressed in human brain. Brain Res. Mol. Brain Res., 1999, 64(2), 193-198.

[489] Baker, D.; Pryce, G.; Davies, W.L.; Hiley, C.R. In silico patent searching reveals a new cannabinoid receptor. Trends Pharmacol. Sci., 2006, 27(1), 1-4

[490] Shore, D.M.; Reggio, P.H. The therapeutic potential of orphan GPCRs, GPR35 and GPR55. Front. Pharmacol., 2015, 6, 69.

[491] Henstridge, C.M.; Balenga, N.A.; Ford, L.A.; Ross, R.A.; Waldhoer, M.; Irving, A.J. The GPR55 ligand L- $\alpha$-lysophosphatidylinositol promotes RhoA-dependent $\mathrm{Ca}^{2+}$ signaling and NFAT activation. FASEB Journal, 2009, 23(1), 183-193.

[492] Lauckner, J.E.; Jensen, J.B.; Chen, H.Y.; Lu, H.C.; Hille, B.; Mackie, K. GPR55 is a cannabinoid receptor that increases intracellular calcium and inhibits M current. Proc. Natl. Acad. Sci. USA, 2008, 105(7), 2699-2704.

[493] Henstridge, C.M.; Balenga, N.A.; Schröder, R.; Kargl, J.K.; Platzer, W.; Martini, L.; Arthur, S.; Penman, J.; Whistler, J.L.; Kostenis, E.; Waldhoer, M.; Irving, A.J. GPR55 ligands promote receptor coupling to multiple signalling pathways. Br. J. Pharmacol., 2010; 160, 604614.

[494] Kapur, A.; Zhao, P.; Sharir, H.; Bai, Y.; Caron, M.G.; Barak, L.S.; Abood, M.E. Atypical responsiveness of the orphan receptor GPR55 to cannabinoid ligands. J. Biol. Chem., 2009, 284, 29817-29827.

[495] Oka, S.; Kimura, S.; Toshida, T.; Ota, R.; Yamashita, A.; Sugiura, T. Lysophosphatidylinositol induces rapid phosphorylation of p38 mitogen-activated protein kinase and activating transcription factor 2 in HEK293 cells expressing GPR55 and IM-9 lymphoblastoid cells. J. Biochem., 2010, 147(5), 671-678.

[496] Oka, S.; Nakajima, K.; Yamashita, A.; Kishimoto, S.; Sugiura, T. Identification of GPR55 as a lysophosphatidylinositol receptor. Biochem. Biophys. Res. Commun., 2007, 362(4), 928-934.

[497] Oka, S.; Toshida, T.; Maruyama, K.; Nakajima, K.; Yamashita, A.; Sugiura, T. 2-Arachidonoyl-sn-glycero-3-phosphoinositol: a possible natural ligand for GPR55. J. Biochem., 2009, 145(1), 13-20.

[498] Guy, A.T.; Nagatsuka, Y.; Ooashi, N.; Inoue, M.; Nakata, A.; Greimel, P.; Inoue, A.; Nabetani, T.; Murayama, A.; Ohta, K.; Ito, Y.; Aoki, J.; Hirabayashi, Y.; Kamiguchi, H. Glycerophospholipid regulation of modality-specific sensory axon guidance in the spinal cord. Science, 2015, 349(6251), 974-977.

[499] Overton, H.A.; Babbs, A.J.; Doel, S.M.; Fyfe, M.C.; Gardner, L.S.; Griffin, G.; Jackson, H.C.; Procter, M.J.; Rasamison, C.M.; TangChristensen, M.; Widdowson, P.S.; Williams, G.M.; Reynet, C. Deorphanization of a G protein-coupled receptor for oleoylethanolamide and its use in the discovery of small-molecule hypophagic agents. Cell. Metab., 2006, 3(3), 167-175.

[500] Waldeck-Weiermair, M.; Zoratti, C.; Osibow, K.; Balenga, N.; Goessnitzer, E.; Waldhoer, M.; Malli, R.; Graier, W.F. Integrin clustering enables anandamide-induced $\mathrm{Ca}^{2+}$ signalling in endothelial cells via GPR55 by protection against $\mathrm{CB}_{1}$-receptor-triggered repression. J. Cell. Sci., 2008, 121, 1704-1717.
[501] Nevalainen, T.; Irving, A.J. GPR55, a lysophosphatidylinositol receptor with cannabinoid sensitivity? Curr. Top. Med. Chem., 2010, 10(8), 799-813.

[502] Sharir, H.; Console-Bram, L.; Mundy, C.; Popoff, S.N.; Kapur, A.; Abood, M.E. The endocannabinoids anandamide and virodhamine modulate the activity of the candidate cannabinoid receptor GPR55. J. Neuroimmune Pharmacol., 2012, 7(4), 856-865.

[503] Sharir, H.; Abood, M.E. Pharmacological characterization of GPR55, a putative cannabinoid receptor. Pharmacol. Ther., 2010, 126, 301313.

[504] Henstridge, C.M. Off-target cannabinoid effects mediated by GPR55. Pharmacology, 2012, 89(3-4), 179-187.

[505] Ross, R.A. The enigmatic pharmacology of GPR55. Trends in Pharmacological Sciences, 2009, 30(3), 156-163.

[506] Anavi-Goffer, S.; Baillie, G.; Irving, A.J.; Gertsch, J.; Greig, I.R.; Pertwee, R.G.; Ross, R.A. Modulation of L- $\alpha-$ lysophosphatidylinositol/GPR55 mitogen-activated protein kinase (MAPK) signaling by cannabinoids. J. Biol. Chem., 2012, 287(1), 91104.

[507] Anavi-Goffer, S.; Irving, A.J; Ross, R.A. Modulation of l- $\alpha$ lysophosphatidylinositol/GPR55 MAP kinase signalling by $\mathrm{CB}_{2}$ receptor agonists: identifying novel GPR55 inhibitors. J. Basic Clin. Physiol. Pharmacol., 2016, 27(3), 303-310.

[508] Vollner, L.; Bieniek, D.; Korte, F. Haschisch XX. Cannabidivarin, ein neuer Haschisch-Inhaltsstoff. Tetrahedron Lett., 1969, 3, 145147.

[509] Köfalvi, A. In: Cannabinoids and the Brain; Köfalvi, A., Ed. Springer US, 2008, pp. 131-160.

[510] Kargl, J.; Brown, A.J.; Andersen, L.; Dorn, G.; Schicho, R.; Waldhoer, M.; Heinemann, A. A selective antagonist reveals potential role of $\mathrm{G}$ protein-coupled receptor 55 in platelet and endothelial cell function. J. Pharmacol. Exp. Ther., 2013, 346(1), 5466.

[511] Heynen-Genel, S.; Dahl, R.; Shi, S.; Milan, L.; Hariharan, S.; Bravo, Y.; Sergienko, E.; Hedrick, M.; Dad, S.; Stonich, D.; Su, Y.; Vicchiarelli, M.; Mangravita-Novo, A.; Smith, L.H.; Chung, T.D.; Sharir, H.; Barak, L.S.; Abood, M.E. Screening for Selective Ligands for GPR55 - Agonists. Probe Reports from the NIH Molecular Libraries Program [Internet]. Bethesda (MD): National Center for Biotechnology Information (US); 2010-. Available from: http://www.ncbi.nlm.nih.gov/books/NBK66153/ (Accessed October 15, 2015).

[512] Nilius, B.; Szallasi, A. Transient receptor potential channels as drug targets: from the science of basic research to the art of medicine. Pharmacol. Rev., 2014, 66(3), 676-814.

[513] Caterina, M.J.; Schumacher, M.A.; Tominaga, M.; Rosen, T.A.; Levine, J.D.; Julius, D. The capsaicin receptor: a heat-activated ion channel in the pain pathway. Nature, 1997, 389, 816-824.

[514] Caterina, M.J.; Leffler, A.; Malmberg, A.B.; Martin, W.J.; Trafton, J.; Petersen-Zeitz, K.R.; Koltzenburg, M.; Basbaum, A.I.; Julius, D. (2000) Impaired nociception and pain sensation in mice lacking the capsaicin receptor. Science, 2000, 288, 306-313.

[515] Starowicz, K.; Nigam, S.; Di Marzo, V. Biochemistry and pharmacology of endovanilloids. Pharmacol. Ther., 2007, 114, 1333.

[516] Zygmunt, P.M.; Petersson, J.; Andersson, D.A.; Chuang, H.; Sørgård, M.; Di Marzo, V.; Julius, D.; Högestätt, E.D. Vanilloid receptors on sensory nerves mediate the vasodilator action of anandamide. Nature, 1999, 400, 452-457.

[517] Huang, S.M.; Bisogno, T.; Trevisani, M.; Al-Hayani, A.; De Petrocellis, L.; Fezza, F.; Tognetto, M.; Petros, T.J.; Krey, J.F.; Chu, C.J.; Miller, J.D.; Davies, S.N.; Geppetti, P.; Walker, J.M.; Di Marzo, V. An endogenous capsaicin-like substance with high potency at recombinant and native vanilloid VR1 receptors. Proc. Natl. Acad. Sci. USA, 2002, 99(12), 8400-8405.

[518] Zygmunt, P.M.; Ermund, A.; Movahed, P.; Andersson, D.A.; Simonsen, C.; Jönsson, B.A.; Blomgren, A.; Birnir, B.; Bevan, S.; Eschalier, A.; Mallet, C.; Gomis, A.; Högestätt, E.D. Monoacylglycerols activate $\mathrm{TRPV}_{1}$ - a link between phospholipase $\mathrm{C}$ and TRPV. . PLOS ONE, 2013, 8(12), e81618.

[519] Cao, E.; Liao, M.; Cheng, Y.; Julius, D. TRPV ${ }_{1}$ structures in distinct conformations reveal activation mechanisms. Nature, 2013, 504, 113118.

[520] Liao, M.; Cao, E.; Julius, D.; Cheng, Y. Structure of the TRPV 1 ion channel determined by electron cryo-microscopy. Nature, 2013, 504 107-112.

[521] Hofmann, N.A.; Barth, S.; Waldeck-Weiermair, M.; Klec, C.; Strunk, D.; Malli, R.; Graier, W.F. TRPV 1 mediates cellular uptake of 
anandamide and thus promotes endothelial cell proliferation and network-formation. Biol. Open., 2014, 3(12), 1164-1172.

[522] Ligresti, A.; Moriello, A.S.; Starowicz, K.; Matias, I.; Pisanti, S.; De Petrocellis, L.; Laezza, C.; Portella, G.; Bifulco, M.; Di Marzo, V. Antitumor activity of plant cannabinoids with emphasis on the effect of cannabidiol on human breast carcinoma. J. Pharmacol. Exp. Ther., 2006, 318(3), 1375-1387.

[523] Szallasi, A. Piperine: researchers discover new flavor in an ancient spice. Trends Pharmacol. Sci., 2005, 26(9), 437-439.

[524] Qin, N.; Neeper, M.P.; Liu, Y.; Hutchinson, T.L.; Lubin, M.L.; Flores, C.M. TRPV 2 is activated by cannabidiol and mediates CGRP release in cultured rat dorsal root ganglion neurons. J. Neurosci., 2008, 28, 6231-6238.

[525] Gibson, H.E.; Edwards, J.G.; Page, R.S.; van Hook, M.J.; Kauer, J.A. $\mathrm{TRPV}_{1}$ channels mediate long-term depression at synapses on hippocampal interneurons. Neuron, 2008, 57, 746-759.

[526] Jin, K.; Xie, L.; Kim, S.H.; Parmentier-Batteur, S.; Sun, Y.; Mao, X.O.; Childs, J.; Greenberg, D.A. Defective adult neurogenesis in $\mathrm{CB}_{1}$ cannabinoid receptor knockout mice. Mol. Pharmacol., 2004, 66(2), 204-208.

[527] Micale, V.; Di Marzo, V.; Sulcova, A.; Wotjak, C.T.; Drago, F. Endocannabinoid system and mood disorders: priming a target for new therapies. Pharmacol. Ther., 2013, 138(1), 18-37.

[528] Köles, L.; Garção, P.; Zádori, Z.S.; Ferreira, S.G.; Pinheiro, B.S.; da Silva-Santos, C.S.; Ledent, C.; Köfalvi, A. Presynaptic TRPV 1 vanilloid receptor function is age- but not $\mathrm{CB}_{1}$ cannabinoid receptordependent in the rodent forebrain. Brain Res. Bull., 2013, 97, 126135.

[529] Maccarrone, M.; Rossi, S.; Bari, M.; De Chiara, V.; Fezza, F.; Musella, A.; Gasperi, V.; Prosperetti, C.; Bernardi, G.; Finazzi-Agrò, A.; Cravatt, B.F.; Centonze, D. Anandamide inhibits metabolism and physiological actions of 2-arachidonoylglycerol in the striatum. Nat. Neurosci., 2008, 11(2), 152-159.

[530] Lee, S.H.; Ledri, M.; Tóth, B.; Marchionni, I.; Henstridge, C.M.; Dudok, B.; Kenesei, K.; Barna, L.; Szabó, S.I.; Renkecz, T.; Oberoi, M.; Watanabe, M.; Limoli, C.L.; Horvai, G.; Soltesz, I.; Katona, I. Multiple forms of endocannabinoid and endovanilloid signaling regulate the tonic control of GABA release. J. Neurosci., 2015, 35(27), 10039-10057.

[531] Jordt, S.-E.; Bautista, D.M.; Chuang, H.-h.; McKemy, D.D.; Zygmunt, P.M.; Hogestatt, E.D.; Meng, I.D.; Julius, D. Mustard oils and cannabinoids excite sensory nerve fibres through the TRP channel ANKTM1. Nature, 2004, 427, 260-265.

[532] Schepers, R.J.; Ringkamp, M. Thermoreceptors and thermosensitive afferents. Neurosci. Biobehav. Rev., 2010, 34(2), 177-184.

[533] De Petrocellis, L.; Vellani, V.; Schiano-Moriello, A.; Marini, P.; Magherini, P.C.; Orlando, P.; Di Marzo, V. Plant-derived cannabinoids modulate the activity of transient receptor potential channels of ankyrin type-1 and melastatin type-8. .J Pharmacol. Exp. Ther., 2008, 325(3), 1007-1015.

[534] Rao, G.K.; Kaminski, N.E. Cannabinoid-mediated elevation of intracellular calcium: a structure-activity relationship. J. Pharmacol. Exp. Ther., 2006, 317, 820-829.

[535] Rao, G.K.; Kaminski, N.E. Induction of intracellular calcium elevation by Delta9-tetrahydrocannabinol in T cells involves TRPC1 channels. J. Leukoc. Biol., 2006, 79, 202-213.

[536] De Petrocellis, L.; Starowicz, K.; Moriello, A.S.; Vivese, M.; Orlando, P.; Di Marzo, V. Regulation of transient receptor potential channels of melastatin type $8\left(\mathrm{TRPM}_{8}\right)$ : effect of cAMP, cannabinoid $\mathrm{CB}_{1}$ receptors and endovanilloids. Exp. Cel.l Res., 2007, 313(9), 1911-1920.

[537] Zhao, P.; Abood, M.E. GPR55 and GPR35 and their relationship to cannabinoid and lysophospholipid receptors. Life Sci., 2013, 92(8-9), 453-457.

[538] Drzazga, A.; Sowińska, A.; Koziołkiewicz, M. Lysophosphatidylcholine and lysophosphatidylinosiol--novel promissing signaling molecules and their possible therapeutic activity. Acta Pol. Pharm., 2014, 71(6), 887-899.

[539] Brown, A.J. Novel cannabinoid receptors. Br. J. Pharmacol., 2007, 152(5), 567-575.

[540] Godlewski, G.; Offertáler, L.; Wagner, J.A.; Kunos, G. Receptors for acylethanolamides-GPR55 and GPR119. Prostaglandins Other Lipid Mediat., 2009, 89(3-4), 105-111.

[541] Lenkey, N.; Kirizs, T.; Holderith, N.; Máté, Z.; Szabó, G.; Vizi, E.S.; Hájos, N.; Nusser, Z. Tonic endocannabinoid-mediated modulation of
GABA release is independent of the CB1 content of axon terminals. Nat. Commun., 2015, 6, 6557.

[542] Herkenham, M.; Lynn, A.B.; Little, M.D.; Johnson, M.R.; Melvin, L.S.; de Costa, B.R.; Rice, K.C. Cannabinoid receptor localization in brain. Proc. Natl. Acad. Sci. USA, 1990, 87(5), 1932-1936.

[543] Herkenham, M.; Lynn, A.B.; Johnson, M.R.; Melvin, L.S.; De Costa, B.R.; Rice, K.C. Characterization and localization of cannabinoid receptors in rat brain: a quantitative in vitro autoradiographic study. $J$. Neurosci., 1991, 11,563-583.

[544] Mailleux, P.; Vanderhaeghen, J.J. Distribution of neuronal cannabinoid receptor in the adult rat brain: a comparative receptor binding radioautography and in situ hybridization histochemistry. Neuroscience, 1992, 48, 655-668.

[545] Mailleux, P.; Parmentier, M.; Vanderhaeghen, J.J. Distribution of cannabinoid receptor messenger RNA in the human brain: an in situ hybridization histochemistry with oligonucleotides. Neurosci. Lett., 1992, 143, 200-204.

[546] Glass, M.; Dragunow, M.; Faull, R.L. Cannabinoid receptors in the human brain: a detailed anatomical and quantitative autoradiographic study in the fetal, neonatal and adult human brain. Neuroscience, 1997, 77, 299-318.

[547] Tsou, K.; Brown, S.; Sañudo-Peña, M.C.; Mackie, K.; Walker, J.M. Immunohistochemical distribution of cannabinoid CB1 receptors in the rat central nervous system. Neuroscience, 1998, 83(2), 393-411.

[548] Marsicano, G.; Lutz, B. Expression of the cannabinoid receptor CB in distinct neuronal subpopulations in the adult mouse forebrain. Eur. J. Neurosci., 1999, 11, 4213-4225.

[549] Egertová, M.; Elphick, M.R. Localisation of cannabinoid receptors in the rat brain using antibodies to the intracellular C-terminal tail of $\mathrm{CB}_{1}$. J. Comp. Neurol., 2000, 422, 159-171.

[550] Mackie, K. Distribution of cannabinoid receptors in the central and peripheral nervous system. Handb. Exp. Pharmacol., 2005, 299-325.

[551] Hollister, L.E. Health aspects of Cannabis. Pharmacol. Rev., 1986, 38(1), 1-20.

[552] Uchigashima, M.; Narushima, M.; Fukaya, M.; Katona, I.; Kano, M.; Watanabe, M. Subcellular arrangement of molecules for 2arachidonoyl-glycerol-mediated retrograde signaling and its physiological contribution to synaptic modulation in the striatum. $J$. Neurosci., 2007, 27(14), 3663-3676.

[553] Ferreira, S.G.; Gonçalves, F.Q.; Marques, J.M.; Tomé, Â.R.; Rodrigues, R.J.; Nunes-Correia, I.; Ledent, C.; Harkany, T.; Venance, L.; Cunha, R.A.; Köfalvi, A. Presynaptic adenosine $A_{2 A}$ receptors dampen cannabinoid $\mathrm{CB}_{1}$ receptor-mediated inhibition of corticostriatal glutamatergic transmission. Br. J. Pharmacol., 2015, 172(4), 1074-1086.

[554] Katona, I.; Sperlágh, B.; Sík, A.; Köfalvi, A.; Vizi, E.S.; Mackie, K.; Freund, T.F. Presynaptically located $\mathrm{CB}_{1}$ cannabinoid receptors regulate GABA release from axon terminals of specific hippocampal interneurons. J. Neurosci., 1999, 19(11), 4544-4558.

[555] Savonenko, A.V.; Melnikova, T.; Wang, Y.; Ravert, H.; Gao, Y.; Koppel, J.; Lee, D.; Pletnikova, O.; Cho, E.; Sayyida, N.; Hiatt, A.; Troncoso, J.; Davies, P.; Dannals, R.F.; Pomper, M.G.; Horti, A.G. Cannabinoid $\mathrm{CB}_{2}$ receptors in a mouse model of $\mathrm{A} \beta$ amyloidosis: Immunohistochemical analysis and suitability as a PET biomarker of neuroinflammation. PLOS ONE, 2015, 10(6), e0129618.

[556] Li, Y.; Kim, J. Neuronal expression of $\mathrm{CB}_{2}$ cannabinoid receptor mRNAs in the mouse hippocampus. Neuroscience, 2015, 311, 253267.

[557] Andó, R.D.; Bíró, J.; Csölle, C.; Ledent, C.; Sperlágh, B. The inhibitory action of exo- and endocannabinoids on $\left[{ }^{3} \mathrm{H}\right] \mathrm{GABA}$ release are mediated by both $\mathrm{CB}_{1}$ and $\mathrm{CB}_{2}$ receptors in the mouse hippocampus. Neurochem. Int., 2012, 60(2), 145-152.

[558] Morgan, N.H.; Stanford, I.M.; Woodhall, G.L. Functional $\mathrm{CB}_{2}$ type cannabinoid receptors at CNS synapses. Neuropharmacology, 2009, 57(4), 356-368.

[559] den Boon, F.S.; Chameau, P.; Schaafsma-Zhao, Q.; van Aken, W.; Bari, M.; Oddi, S.; Kruse, C.G.; Maccarrone, M.; Wadman, W.J.; Werkman, T.R. Excitability of prefrontal cortical pyramidal neurons is modulated by activation of intracellular type- 2 cannabinoid receptors. Proc. Natl. Acad. Sci. USA, 2012, 109(9), 3534-3539. 
[560] den Boon, F.S.; Chameau, P.; Houthuijs, K.; Bolijn, S.; Mastrangelo, N.; Kruse, C.G.; Maccarrone, M.; Wadman, W.J.; Werkman, T.R. Endocannabinoids produced upon action potential firing evoke a $\mathrm{Cl}^{-}$ current via type-2 cannabinoid receptors in the medial prefrontal cortex. Pflugers Arch., 2014, 466(12), 2257-2268.

[561] Stempel, A.V.; Stumpf, A.; Zhang, H.Y.; Özdoğan, T.; Pannasch, U.; Theis, A.K.; Otte, D.M.; Wojtalla, A.; Rácz, I.; Ponomarenko, A.; Xi, Z.X.; Zimmer, A.; Schmitz, D. Cannabinoid type 2 receptors mediate a cell type-specific plasticity in the hippocampus. Neuron, 2016, 90(4), 795-809.

[562] Köfalvi, A.; Lemos, C.; Martín-Moreno, A.M.; Pinheiro, B.S.; García-García, L.; Pozo, M.A.; Valério-Fernandes, Â.; Beleza, R.O.; Agostinho, P.; Rodrigues, R.J.; Pasquaré, S.J.; Cunha, R.A.; de Ceballos, M.L. Stimulation of brain glucose uptake by cannabinoid CB2 receptors and its therapeutic potential in Alzheimer's disease. Neuropharmacology, 2016, S0028-3908(16), 30087-30089.

[563] Ashton, J.C. The atypical cannabinoid o-1602: Targets, actions, and the central nervous system. Cent. Nerv. Syst. Agents Med. Chem., 2012, 12(3), 233-239.

[564] Abed, E.; Labelle, D.; Martineau, C.; Loghin, A.; Moreau, R. Expression of transient receptor potential (TRP) channels in human and murine osteoblast-like cells. Mol. Membr. Biol., 2009, 26, 146158.

[565] Rossi, F.; Siniscalco, D.; Luongo, L.; De Petrocellis, L.; Bellini, G.; Petrosino, S.; Torella, M.; Santoro, C.; Nobili, B.; Perrotta, S.; Di Marzo, V.; Maione, S. The endovanilloid/endocannabinoid system in human osteoclasts: possible involvement in bone formation and resorption. Bone, 2009, 44(3), 476-484.

[566] Whyte, L.S.; Ryberg, E.; Sims, N.A.; Ridge, S.A.; Mackie, K.; Greasley, P.J.; Ross, R.A.; Rogers, M.J. The putative cannabinoid receptor GPR55 affects osteoclast function in vitro and bone mass in vivo. Proc. Natl. Acad. Sci. USA, 2009, 106, 16511-16516.

[567] Romero-Zerbo, S.Y.; Rafacho, A.; Díaz-Arteaga, A.; Suárez, J.; Quesada, I.; Imbernon, M.; Ross, R.A.; Dieguez, C.; Rodríguez de Fonseca, F.; Nogueiras, R.; Nadal, A.; Bermúdez-Silva, F.J. A role for the putative cannabinoid receptor GPR55 in the islets of Langerhans. J. Endocrinol., 2011, 211(2), 177-185.

[568] Moreno-Navarrete, J.M.; Catalán, V.; Whyte, L.; Díaz-Arteaga, A.; Vázquez-Martínez, R.; Rotellar, F.; Guzmán, R.; Gómez-Ambrosi, J.; Pulido, M.R.; Russell, W.R.; Imbernón, M.; Ross, R.A.; Malagón, M.M.; Dieguez, C.; Fernández-Real, J.M.; Frühbeck, G.; Nogueiras, R. The L- $\alpha$-lysophosphatidylinositol/GPR55 system and its potential role in human obesity. Diabetes, 2012, 61(2), 281-291.

[569] Sylantyev, S.; Jensen, T.P.; Ross, R.A.; Rusakov, D.A. Cannabinoidand lysophosphatidylinositol-sensitive receptor GPR55 boosts neurotransmitter release at central synapses. Proc. Natl. Acad. Sci. USA, 2013, 110(13), 5193-5198.

[570] Ross, R.A. L- $\alpha$-lysophosphatidylinositol meets GPR55: a deadly relationship. Trends Pharmacol. Sci., 2011, 32(5), 265-269.

[571] Martire, A.; Tebano, M.T.; Chiodi, V.; Ferreira, S.G.; Cunha, R.A.; Köfalvi, A.; Popoli, P. Pre-synaptic adenosine $A_{2 A}$ receptors control cannabinoid $\mathrm{CB}_{1}$ receptor-mediated inhibition of striatal glutamatergic neurotransmission. J. Neurochem., 2011, 116(2), 273280.

[572] Ledent, C.; Valverde, O.; Cossu, G.; Petitet, F.; Aubert, J.F.; Beslot, F.; Böhme, G.A.; Imperato, A.; Pedrazzini, T.; Roques, B.P.; Vassart, G.; Fratta, W.; Parmentier, M. Unresponsiveness to cannabinoids and reduced addictive effects of opiates in $\mathrm{CB}_{1}$ receptor knockout mice. Science, 1999, 283, 401-404.

[573] Ferreira, S.G.; Lomaglio, T.; Avelino, A.; Cruz, F.; Oliveira, C.R.; Cunha, R.A.; Köfalvi, A. N-acyldopamines control striatal input terminals via novel ligand-gated cation channels. Neuropharmacology, 2009, 56(3), 676-683.

[574] Han, J.; Kesner, P.; Metna-Laurent, M.; Duan, T.; Xu, L.; Georges, F.; Koehl, M.; Abrous, D.N.; Mendizabal-Zubiaga, J.; Grandes, P.; Liu, Q.; Bai, G.; Wang, W.; Xiong, L.; Ren, W.; Marsicano, G.; Zhang, X. Acute cannabinoids impair working memory through astroglial $\mathrm{CB}_{1}$ receptor modulation of hippocampal LTD. Cell, 2012, 148(5), 10391050 .

[575] Navarrete, M.; Araque, A. Endocannabinoids potentiate synaptic transmission through stimulation of astrocytes. Neuron, 2010, 68(1), 113-126.
[576] Bosier, B.; Bellocchio, L.; Metna-Laurent, M.; Soria-Gomez, E.; Matias, I.; Hebert-Chatelain, E.; Cannich, A.; Maitre, M.; LesteLasserre, T.; Cardinal, P.; Mendizabal-Zubiaga, J.; Canduela, M.J.; Reguero, L.; Hermans, E.; Grandes, P.; Cota, D.; Marsicano, G. Astroglial $\mathrm{CB}_{1}$ cannabinoid receptors regulate leptin signaling in mouse brain astrocytes. Mol. Metab., 2013, 2(4), 393-404.

[577] Tsou, K.; Mackie, K.; Sañudo-Peña, M.C.; Walker, J.M. Cannabinoid $\mathrm{CB}_{1}$ receptors are localized primarily on cholecystokinin-containing GABAergic interneurons in the rat hippocampal formation. Neuroscience, 1999, 93(3), 969-975.

[578] Katona, I.; Sperlágh, B.; Maglóczky, Z.; Sántha, E.; Köfalvi, A.; Czirják, S.; Mackie, K.; Vizi, E.S.; Freund, T.F. GABAergic interneurons are the targets of cannabinoid actions in the human hippocampus. Neuroscience, 2000, 100(4), 797-804.

[579] Freund, T.F.; Katona, I.; Piomelli, D. Role of endogenous cannabinoids in synaptic signaling. Physiol. Rev., 2003, 83(3), 10171066.

[580] Degroot, A.; Köfalvi, A.; Wade, M.R.; Davis, R.J.; Rodrigues, R.J.; Rebola, N.; Cunha, R.A.; Nomikos, G.G. CB $_{1}$ receptor antagonism increases hippocampal acetylcholine release: site and mechanism of action. Mol. Pharmacol., 2006, 70(4), 1236-1245.

[581] Cachope, R. Functional diversity on synaptic plasticity mediated by endocannabinoids. Philos. T. R. Soc. B., 2012, 367(1607), 3242-3253.

[582] Kano, M. Control of synaptic function by endocannabinoid-mediated retrograde signaling. Proc. Jpn. Acad. Ser. B. Phys. Biol. Sci., 2014, 90(7), 235-250.

[583] Straiker, A.; Mackie, K. Depolarization-induced suppression of excitation in murine autaptic hippocampal neurones. J. Physiol., 2005, 569(2), 501-517.

[584] Straiker, A.; Mackie, K. Metabotropic suppression of excitation in murine autaptic hippocampal neurons. J. Physiol., 2007, 578(3), 773785.

[585] Guo, J.; Ikeda, S.R. Endocannabinoids modulate N-type calcium channels and G-protein-coupled inwardly rectifying potassium channels via $\mathrm{CB}_{1}$ cannabinoid receptors heterologously expressed in mammalian neurons. Mol. Pharmacol., 2004, 65(3), 665-674.

[586] Gerdeman, G.L. In: Cannabinoids and the Brain; Köfalvi, A., Ed. Springer US, 2008, pp. 203-236.

[587] Varma, N.; Carlson, G.C.; Ledent, C.; Alger, B.E. Metabotropic glutamate receptors drive the endocannabinoid system in hippocampus. J. Neurosci., 2001, 21(24), RC188.

[588] Heifets, B.D.; Chevaleyre, V.; Castillo, P.E. Interneuron activity controls endocannabinoid-mediated presynaptic plasticity through calcineurin. Proc. Natl. Acad. Sci. USA, 2008, 105(29), 10250-10255.

[589] Zachariou, M.; Alexander, S.P.; Coombes, S.; Christodoulou, C. A biophysical model of endocannabinoid-mediated short term depression in hippocampal inhibition. PLoS ONE, 2013, 8(3), e58926.

[590] Kreitzer, A.C.; Regehr, W.G. Retrograde inhibition of presynaptic calcium influx by endogenous cannabinoids at excitatory synapses onto Purkinje cells. Neuron, 2001, 29, 717-727.

[591] Ohno-Shosaku, T.; Maejima, T.; Kano; M. Endogenous cannabinoids mediate retrograde signals from depolarized postsynaptic neurons to presynaptic terminals. Neuron, 2001, 29, 729-738.

[592] Wilson, R.I.; Nicoll, R.A. Endogenous cannabinoids mediate retrograde signalling at hippocampal synapses. Nature, 2001, 410, 588-592.

[593] Hashimotodani, Y.; Ohno-Shosaku, T.; Tsubokawa, H.; Ogata, H.; Emoto, K.; Maejima, T.; Araishi, K.; Shin, H.S.; Kano, M. Phospholipase $C \beta$ serves as a coincidence detector through its $\mathrm{Ca}^{2+}$ dependency for triggering retrograde endocannabinoid signal. Neuron, 2005, 45, 257-268.

[594] Hashimotodani, Y.; Ohno-Shosaku, T.; Watanabe, M.; Kano, M. Roles of phospholipase Cbeta and NMDA receptor in activitydependent endocannabinoid release. J. Physiol., 2007, 584(2), 373380.

[595] Chevaleyre, V.; Heifets, B.D.; Kaeser, P.S.; Südhof, T.C.; Castillo, P.E. Endocannabinoid-mediated long-term plasticity requires cAMP/PKA signaling and RIM1 $\alpha$. Neuron, 2007, 54(5), 801-812. 
[596] Tsetsenis, T.; Younts, T.J.; Chiu, C.Q.; Kaeser, P.S.; Castillo, P.E.; Südhof, T.C. Rab3B protein is required for long-term depression of hippocampal inhibitory synapses and for normal reversal learning. Proc. Natl. Acad. Sci. USA, 2011, 108(34), 14300-14305.

[597] Caporale, N.; Dan, Y. Spike timing-dependent plasticity: a Hebbian learning rule. Annu. Rev. Neurosci., 2008, 31, 25-46.

[598] Cui, Y.; Paillé, V.; Xu, H.; Genet, S.; Delord, B.; Fino, E.; Berry, H.; Venance, L. Endocannabinoids mediate bidirectional striatal spiketiming-dependent plasticity. J. Physiol., 2015, 593(13), 2833-2849.

[599] Cui, Y.; Prokin, I.; Xu, H.; Delord, B.; Genet, S.; Venance, L.; Berry, H. Endocannabinoid dynamics gate spike-timing dependent depression and potentiation. Elife, 2016, 5, e13185.

[600] Marinelli, S.; Pacioni, S.; Cannich, A.; Marsicano, G.; Bacci, A. Selfmodulation of neocortical pyramidal neurons by endocannabinoids. Nat. Neurosci., 2009, 12(12), 1488-1490.

[601] Bacci, A.; Huguenard, J.R.; Prince, D.A. Long-lasting self-inhibition of neocortical interneurons mediated by endocannabinoids. Nature, 2004, 431(7006), 312-316.

[602] Maroso, M.; Szabo, G.G.; Kim, H.K.; Alexander, A.; Bui, A.D.; Lee, S.H.; Lutz, B.; Soltesz, I. Cannabinoid control of learning and memory through HCN channels. Neuron, 2016, 89(5), 1059-1073.

[603] Pape, H.C. Queer current and pacemaker: the hyperpolarizationactivated cation current in neurons. Annu. Rev. Physiol., 1996, 58, 299-327.

[604] Nolan, M.F.; Malleret, G.; Dudman, J.T.; Buhl, D.L.; Santoro, B.; Gibbs, E.; Vronskaya, S.; Buzsáki, G.; Siegelbaum, S.A.; Kandel, E.R.; Morozov, A. A behavioral role for dendritic integration: HCN1 channels constrain spatial memory and plasticity at inputs to distal dendrites of CA1 pyramidal neurons. Cell, 2004, 119, 719-732.

[605] Shah, M.M. Cortical HCN channels: function, trafficking and plasticity. J. Physiol., 2014, 592, 2711-2719.

[606] Whiting, P.F.; Wolff, R.F.; Deshpande, S.; Di Nisio, M.; Duffy, S.; Hernandez, A.V.; Keurentjes, J.C.; Lang, S.; Misso, K.; Ryder, S.; Schmidlkofer, S.; Westwood, M.; Kleijnen, J. Cannabinoids for Medical Use: A Systematic Review and Meta-analysis. JAMA, 2015, 313(24), 2456-2473.

[607] Eisenstein, M. Medical marijuana: Showdown at the cannabis corral. Nature, 2015, 525(7570), S15-S17.

[608] Page, J.; Ware, M. Perspective: Close the knowledge gap. Nature, 2015, 525(7570), S9.

[609] Alexander, S.P. Therapeutic potential of cannabis-related drugs. Prog. Neuropsychopharmacol. Biol. Psychiatry, 2016, 64, 157-166.

[610] Pernía-Andrade, A.J.; Kato, A.; Witschi, R.; Nyilas, R.; Katona, I.; Freund, T.F.; Watanabe, M.; Filitz, J.; Koppert, W.; Schüttler, J.; Ji, G.; Neugebauer, V.; Marsicano, G.; Lutz, B.; Vanegas, H.; Zeilhofer, H.U. Spinal endocannabinoids and $\mathrm{CB}_{1}$ receptors mediate C-fiberinduced heterosynaptic pain sensitization. Science, 2009, 325(5941), 760-764.

[611] Piomelli, D.; Hohmann, A.G.; Seybold, V.; Hammock, B.D. A lipid gate for the peripheral control of pain. J. Neurosci., 2014, 34(46), 15184-15191.

[612] Woodhams, S.G.; Sagar, D.R.; Burston, J.J.; Chapman, V. The role of the endocannabinoid system in pain. Handb. Exp. Pharmacol., 2015, 227, 119-143.

[613] Kaplan, B.L. The role of $\mathrm{CB}_{1}$ in immune modulation by cannabinoids. Pharmacol. Ther., 2013, 137(3), 365-374.

[614] Rom, S.; Persidsky, Y. Cannabinoid receptor 2: potential role in immunomodulation and neuroinflammation. J. Neuroimmune Pharmacol., 2013, 8(3), 608-620.

[615] Suárez-Pinilla, P.; López-Gil, J.; Crespo-Facorro, B. Immune system: a possible nexus between cannabinoids and psychosis. Brain Behav. Immun., 2014, 40, 269-282.

[616] Centonze, D.; Finazzi-Agrò, A.; Bernardi, G.; Maccarrone, M. The endocannabinoid system in targeting inflammatory neurodegenerative diseases. Trends Pharmacol. Sci., 2007, 28(4), 180-187.

[617] Wang, D.; Couture, R.; Hong, Y. Activated microglia in the spinal cord underlies diabetic neuropathic pain. Eur. J .Pharmacol., 2014, 728, 59-66.
[618] Di Marzo, V.; Stella, N.; Zimmer, A. Endocannabinoid signalling and the deteriorating brain. Nat. Rev. Neurosci., 2015, 16(1), 30-42.

[619] Avraham, Y.; Davidi, N.; Porat, M.; Chernoguz, D.; Magen, I.; Vorobeiv, L.; Berry, E.M.; Leker, R.R. Leptin reduces infarct size in association with enhanced expression of $\mathrm{CB}_{2}, \mathrm{TRPV}_{1}$, SIRT-1 and leptin receptor. Curr. Neurovasc. Res., 2010, 7(2), 136-143.

[620] Viscomi, M.T.; Oddi, S.; Latini, L.; Bisicchia, E.; Maccarrone, M.; Molinari, M. The endocannabinoid system: a new entry in remote cell death mechanisms. Exp. Neurol., 2010, 224(1), 56-65.

[621] Fernández-Ruiz, J.; Moro, M.A.; Martínez-Orgado, J. Cannabinoids in neurodegenerative disorders and stroke/brain trauma: From preclinical models to clinical applications. Neurotherapeutics, 2015, 12(4), 793-806.

[622] Rossi, S.; Furlan, R.; De Chiara, V.; Muzio, L.; Musella, A.; Motta, C.; Studer, V.; Cavasinni, F.; Bernardi, G.; Martino, G.; Cravatt, B.F.; Lutz, B.; Maccarrone, M.; Centonze, D. Cannabinoid $\mathrm{CB}_{1}$ receptors regulate neuronal TNF- $\alpha$ effects in experimental autoimmune encephalomyelitis. Brain Behav. Immun., 2011, 25(6), 1242-1248.

[623] Pryce, G.; Visintin, C.; Ramagopalan, S.V.; Al-Izki, S.; De Faveri, L.E.; Nuamah, R.A.; Mein, C.A.; Montpetit, A.; Hardcastle, A.J.; Kooij, G.; de Vries, H.E.; Amor, S.; Thomas, S.A.; Ledent, C.; Marsicano, G.; Lutz, B.; Thompson, A.J.; Selwood, D.L.; Giovannoni, G.; Baker, D. Control of spasticity in a multiple sclerosis model using central nervous system-excluded CB1 cannabinoid receptor agonists. FASEB J., 2014, 28(1), 117-130.

[624] Pryce, G.; Baker, D. Endocannabinoids in Multiple Sclerosis and Amyotrophic Lateral Sclerosis. Handb. Exp. Pharmacol., 2015, 231, 213-231.

[625] Bilkei-Gorzó, A. The endocannabinoid system in normal and pathological brain ageing. Philos. T. R. Soc. B., 2012, 367(1607), 3326-3341.

[626] Vinod, K.Y.; Arango, V.; Xie, S.; Kassir, S.A.; Mann, J.J.; Cooper, T.B.; Hungund, B.L. Elevated levels of endocannabinoids and $\mathrm{CB}_{1}$ receptor-mediated G-protein signaling in the prefrontal cortex of alcoholic suicide victims. Biol. Psychiatry, 2005, 57(5), 480-486.

[627] Vinod, K.Y. In: The Neurobiological Basis of Suicide; Dwivedi, Y., Ed. CRC Press: Boca Raton (FL), 2012. pp. 87-112.

[628] Avraham, Y.; Grigoriadis, N.; Poutahidis, T.; Vorobiev, L.; Magen, I.; Ilan, Y.; Mechoulam, R.; Berry, E. Cannabidiol improves brain and liver function in a fulminant hepatic failure-induced model of hepatic encephalopathy in mice. Br. J. Pharmacol., 2011, 162(7), 1650-1658.

[629] Tam, J.; Liu, J.; Mukhopadhyay, B.; Cinar, R.; Godlewski, G.; Kunos, G. Endocannabinoids in liver disease. Hepatology, 2011, 53(1), 346-355.

[630] Alswat, K.A. The role of endocannabinoids system in fatty liver disease and therapeutic potentials. Saudi J. Gastroenterol., 2013, 19(4), 144-151.

[631] Bellocchio, L.; Lafenêtre, P.; Cannich, A.; Cota, D.; Puente, N.; Grandes, P.; Chaouloff, F.; Piazza, P.V.; Marsicano, G. Bimodal control of stimulated food intake by the endocannabinoid system. Nat. Neurosci., 2010, 13(3), 281-283.

[632] Scherma, M.; Fattore, L.; Castelli, M.P.; Fratta, W.; Fadda, P. The role of the endocannabinoid system in eating disorders: neurochemical and behavioural preclinical evidence. Curr. Pharm. Des., 2014, 20(13), 2089-2099.

[633] Soria-Gómez, E.; Bellocchio, L.; Marsicano, G. New insights on food intake control by olfactory processes: the emerging role of the endocannabinoid system. Mol. Cell. Endocrinol., 2014, 397(1-2), 5966.

[634] Koch, M.; Varela, L.; Kim, J.G.; Kim, J.D.; Hernández-Nuño, F.; Simonds, S.E.; Castorena, C.M.; Vianna, C.R.; Elmquist, J.K.; Morozov, Y.M.; Rakic, P.; Bechmann, I.; Cowley, M.A.; SzigetiBuck, K.; Dietrich, M.O.; Gao, X.B.; Diano, S.; Horvath, T.L. Hypothalamic POMC neurons promote cannabinoid-induced feeding. Nature, 2015, 519(7541), 45-50.

[635] Cardinal, P.; Bellocchio, L.; Clark, S.; Cannich, A.; Klugmann, M.; Lutz, B.; Marsicano, G.; Cota, D. Hypothalamic CB1 cannabinoid receptors regulate energy balance in mice. Endocrinology, 2012, 153(9), 4136-4143. 
[636] Silvestri, C.; Di Marzo, V. The endocannabinoid system in energy homeostasis and the etiopathology of metabolic disorders. Cell Metab., 2013, 17(4), 475-490.

[637] Gatta-Cherifi, B.; Cota, D. New insights on the role of the endocannabinoid system in the regulation of energy balance. Int. J. Obes. (Lond)., 2016, 40(2), 210-219.

[638] Bénard, G.; Massa, F.; Puente, N.; Lourenço, J.; Bellocchio, L.; Soria-Gómez, E.; Matias, I.; Delamarre, A.; Metna-Laurent, M.; Cannich, A.; Hebert-Chatelain, E.; Mulle, C.; Ortega-Gutiérrez, S.; Martín-Fontecha, M.; Klugmann, M.; Guggenhuber, S.; Lutz, B.; Gertsch, J.; Chaouloff, F.; López-Rodríguez, M.L.; Grandes, P.; Rossignol, R.; Marsicano, G. Mitochondrial $\mathrm{CB}_{1}$ receptors regulate neuronal energy metabolism. Nat. Neurosci., 2012, 15, 558-564.

[639] Lipina, C.; Irving, A.J.; Hundal, H.S. Mitochondria: a possible nexus for the regulation of energy homeostasis by the endocannabinoid system? Am. J. Physiol. Endocrinol. Metab., 2014, 307(1), E1-13.

[640] Ma, L.; Jia, J.; Niu, W.; Jiang, T.; Zhai, Q.; Yang, L.; Bai, F.; Wang, Q.; Xiong, L. Mitochondrial $\mathrm{CB}_{1}$ receptor is involved in ACEAinduced protective effects on neurons and mitochondrial functions. Sci. Rep., 2015, 5, 12440.

[641] Chen, C.C.; Lee, T.Y.; Kwok, C.F.; Hsu, Y.P.; Shih, K.C.; Lin, Y.J.; Ho, L.T. Cannabinoid receptor type 1 mediates high-fat diet-induced insulin resistance by increasing forkhead box $\mathrm{O} 1$ activity in a mouse model of obesity. Int. J. Mol. Med., 2016, 37(3), 743-754.

[642] PDQ Cancer Complementary and Alternative Medicine Editorial Board. Cannabis and Cannabinoids (PDQ $\left.{ }^{\circledR}\right)$ : Patient Version. PDQ Cancer Information Summaries [Internet]. National Cancer Institute (US): Bethesda, MD, 2002-. 2015.

[643] PDQ Cancer Complementary and Alternative Medicine Editorial Board. Cannabis and Cannabinoids (PDQ $\left.{ }^{\circledR}\right)$ : Health Professional Version. PDQ Cancer Information Summaries [Internet]. National Cancer Institute (US): Bethesda, MD, 2002-. 2015.

[644] Abrams, D.I.; Guzman, M. Cannabis in cancer care. Clin. Pharmacol. Ther., 2015, 97(6), 575-586.

[645] Fowler, C.J. Delta ${ }^{9}$-tetrahydrocannabinol and cannabidiol as potential curative agents for cancer: A critical examination of the preclinical literature. Clin. Pharmacol. Ther., 2015b, 97(6), 587-596.

[646] Velasco, G.; Hernández-Tiedra, S.; Dávila, D.; Lorente, M. The use of cannabinoids as anticancer agents. Prog. Neuropsychopharmacol. Biol. Psychiatry, 2016, 64, 259-266.

[647] Dunn, S.L.; Wilkinson, J.M.; Crawford, A.; Le Maitre, C.L.; Bunning, R.A. Cannabinoids: novel therapies for arthritis? Future Med. Chem., 2012, 4(6), 713-725.

[648] La Porta, C.; Bura, S.A.; Negrete, R.; Maldonado, R. Involvement of the endocannabinoid system in osteoarthritis pain. Eur. J. Neurosci., 2014, 39(3), 485-500.

[649] Bab, I.; Smoum, R.; Bradshaw, H.; Mechoulam, R. Skeletal lipidomics: regulation of bone metabolism by fatty acid amide family. Br. J. Pharmacol., 2011, 163(7), 1441-1446.

[650] Schuehly, W.; Paredes, J.M.V.; Kleyer, J.; Huefner, A.; AnaviGoffer, S.; Raduner, S.; Altmann, K.-H.; Gertsch, J. Mechanisms of osteoclastogenesis inhibition by a novel class of biphenyl-type cannabinoid $\mathrm{CB}(2)$ receptor inverse agonists. Chem. Biol., 2011, 18(8), 1053-1064.

[651] Gowran, A.; McKayed, K.; Campbell, V.A. The cannabinoid receptor type 1 is essential for mesenchymal stem cell survival and differentiation: implications for bone health. Stem Cells Int., 2013, 2013, 796715.

[652] Samir, S.M.; Malek, H.A. Effect of cannabinoid receptors 1 modulation on osteoporosis in a rat model of different ages. $J$. Physiol. Pharmacol., 2014, 65(5), 687-694.

[653] Calignano, A.; Katona, I.; Désarnaud, F.; Giuffrida, A.; La Rana, G.; Mackie, K.; Freund, T.F.; Piomelli, D. Bidirectional control of airway responsiveness by endogenous cannabinoids. Nature, 2000, 408(6808), 96-101.

[654] Tomida, I.; Pertwee, R.G.; Azuara-Blanco, A. Cannabinoids and glaucoma. Br. J. Ophthalmol., 2004, 88(5), 708-713.

[655] Sun, X.; Xu, C.S.; Chadha, N.; Chen, A.; Liu, J. Marijuana for glaucoma: A recipe for disaster or treatment? Yale J. Biol. Med., 2015, 88(3), 265-269.
[656] Pagano, E.; Montanaro, V.; Di Girolamo, A.; Pistone, A.; Altieri, V.; Zjawiony, J.K.; Izzo, A.A.; Capasso, R. Effect of Non-psychotropic plant-derived cannabinoids on bladder contractility: Focus on cannabigerol. Nat. Prod. Commun., 2015, 10(6), 1009-1012.

[657] Yang, Z.M.; Paria, B.C.; Dey, S.K. Activation of brain-type cannabinoid receptors interferes with preimplantation mouse embryo development. Biol. Reprod., 1996, 55(4), 756-761.

[658] Trabucco, E.; Acone, G.; Marenna, A.; Pierantoni, R.; Cacciola, G.; Chioccarelli, T.; Mackie, K.; Fasano, S.; Colacurci, N.; Meccariello, R.; Cobellis, G.; Cobellis, L. Endocannabinoid system in first trimester placenta: low FAAH and high CB1 expression characterize spontaneous miscarriage. Placenta, 2009, 30(6), 516-522.

[659] Marsicano, G.; Goodenough, S.; Monory, K.; Hermann, H.; Eder, M.; Cannich, A.; Azad, S.C.; Cascio, M.G.; Gutierrez, S.O.; Van, D.; Lopez, R.; Casanova, E.; Schutz, G.; Zieglgänsberger, W.; Di Marzo, V.; Behl, C.; Lutz, B. $\mathrm{CB}_{1}$ cannabinoid receptors and on-demand defense against excitotoxicity. Science, 2003, 302, 84-88.

[660] Chen, K.; Ratzliff, A.; Hilgenberg, L.; Gulyás, A.; Freund, T.F.; Smith, M.; Dinh, T.P.; Piomelli, D.; Mackie, K.; Soltesz, I. Longterm plasticity of endocannabinoid signaling induced by developmental febrile seizures. Neuron, 2003, 39(4), 599-611.

[661] Monory, K.; Massa, F.; Egertová, M.; Eder, M.; Blaudzun, H.; Westenbroek, R.; Kelsch, W.; Jacob, W.; Marsch, R.; Ekker, M.; Long, J.; Rubenstein, J.L.; Goebbels, S.; Nave, K.A.; During, M.; Klugmann, M.; Wölfel, B.; Dodt, H.U.; Zieglgänsberger, W.; Wotjak, C.T.; Mackie, K.; Elphick, M.R.; Marsicano, G.; Lutz, B. The endocannabinoid system controls key epileptogenic circuits in the hippocampus. Neuron, 2006, 51(4), 455-466.

[662] Katona, I.; Freund, T.F. Endocannabinoid signaling as a synaptic circuit breaker in neurological disease. Nat Med., 2008, 14(9), 923930.

[663] Detyniecki, K.; Hirsch, L. Marijuana use in epilepsy: The myth and the reality. Curr. Neurol. Neurosci. Rep., 2015, 15(10), 586.

[664] Katona, I. Cannabis and endocannabinoid signaling in epilepsy. Handb. Exp. Pharmacol., 2015, 231, 285-316.

[665]

http://www.gwpharm.com/GW\%20Pharmaceuticals\%20Announ ces\%20Positive\%20Phase\%203\%20Pivotal\%20Study\%20Results\% 20for\%20Epidiolex\%20cannabidiol.aspx (Accessed 26 June, 2016)

[666] Cools, R.; Nakamura, K.; Daw, N.D. Serotonin and dopamine: unifying affective, activational, and decision functions. Neuropsychopharmacol., 2011, 36(1), 98-113.

[667] Roiser, J.P.; Sahakian, B.J. Hot and cold cognition in depression. CNS Spectr., 2013, 18(3), 139-149.

[668] Patton, G.C.; Coffey, C.; Carlin, J.B.; Degenhardt, L.; Lynskey, M.; Hall, W. Cannabis use and mental health in young people: cohort study. Br. Med. J., 2002, 325, 1195-1198.

[669] Degenhardt, L.; Hall, W.; Lynskey, M. Exploring the association between cannabis use and depression. Addiction, 2003, 98, 14931504.

[670] Boydell, J.; van Os, J.; Caspi, A.; Kennedy, N.; Giouroukou, E.; Fearon, P.; Farrell, M.; Murray, R.M. Trends in cannabis use prior to first presentation with schizophrenia, in South-East London between 1965 and 1999. Psychol. Med., 2006, 36(10), 1441-1446.

[671] Lu, M.L.; Agito, M.D. Cannabinoid hyperemesis syndrome: Marijuana is both antiemetic and proemetic. Cleve. Clin. J. Med., 2015, 82(7), 429-434.

[672] Verweij, K.J.; Agrawal, A.; Nat, N.O.; Creemers, H.E.; Huizink, A.C.; Martin, N.G.; Lynskey, M.T. A genetic perspective on the proposed inclusion of cannabis withdrawal in DSM-5. Psychol. Med., 2013, 43(8), 1713-1722.

[673] Rimonabant: depression and suicide. Prescrire Int. 2009, 18(99):24.

[674] Di Chiara, G.; Imperato, A. Drugs abused by humans preferentially increase synaptic dopamine concentrations in the mesolimbic system of freely moving rats. Proc. Natl. Acad. USA, 1988, 85, 5274-5278.

[675] Wise, R.A. Dopamine, learning and motivation. Nat. Rev. Neurosci., 2004, 5, 483-494.

[676] Hyman, S.E.; Malenka, R.C.; Nestler, E.J. Neural mechanisms of addiction: the role of reward-related learning and memory. Аnnu. Rev. Neurosci., 2006, 29, 565-598. 
[677] Ton, J.M.N.C.; Gerhardt, G.A.; Friedemann, M.; Etgen, A.M.; Rose, G.M.; Sharpless, N.S.; Gardner, E.L. The effects of $\Delta^{9}$ tetrahydrocannabinol on potassium-evoked release of dopamine in the rat caudate nucleus: an in vivo electrochemical and in vivo microdialysis study. Brain Res., 1988, 451, 59-68.

[678] Chen, J.P.; Paredes, W.; Li, J.; Smith, D.; Lowinson, J.; Gardner, E.L. $\Delta^{9}$-tetrahydrocannabinol produces naloxone-blockable enhancement of presynaptic basal dopamine efflux in nucleus accumbens of conscious, freely-moving rats as measured by intracerebral microdialysis. Psychopharmacology (Berl.), 1990, 102, 156-162.

[679] French, E.D. $\Delta^{9}$-tetrahydrocannabinol excites rat VTA dopamine neurons through activation of cannabinoid $\mathrm{CB}_{1}$ but not opioid receptors. Neurosci. Lett., 1997, 226, 159-162.

[680] French, E.D.; Dillon, K.; Wu, X. Cannabinoids excite dopamine neurons in the ventral tegmentum and substantia nigra. Neuroreport, 1997, 8, 649-652.

[681] Tanda, G.; Pontieri, F.E.; Di Chiara, G. Cannabinoid and heroin activation of mesolimbic dopamine transmission by a common $\mu 1$ opioid receptor mechanism. Science, 1997, 276, 2048-2050.

[682] Gessa, G.L.; Melis, M.; Muntoni, A.L.; Diana, M. Cannabinoids activate mesolimbic dopamine neurons by an action on cannabinoid $\mathrm{CB}_{1}$ receptors. Eur. J. Pharmacol., 1998, 341, 39-44.

[683] Malone, D.T.; Taylor, D.A. Modulation by fluoxetine of striatal dopamine release following $\Delta^{9}$-tetrahydrocannabinol: a microdialysis study in conscious rats. Br. J. Pharmacol., 1999, 128, 21-26.

[684] Cheer, J.F.; Wassum, K.M.; Heien, M.L.; Phillips, P.E.; Wightman, R.M. Cannabinoids enhance subsecond dopamine release in the nucleus accumbens of awake rats. J. Neurosci., 2004, 24, 4393-4400.

[685] Cheer, J.F.; Wassum, K.M.; Sombers, L.A.; Heien, M.L.; Ariansen, J.L.; Aragona, B.J.; Phillips, P.E.; Wightman, R.M. Phasic dopamine release evoked by abused substances requires cannabinoid receptor activation. J. Neurosci., 2007, 27(4), 791-795.

[686] Fadda, P.; Scherma, M.; Spano, M.S.; Salis, P.; Melis, V.; Fattore, L.; Fratta, W. Cannabinoid self-administration increases dopamine release in the nucleus accumbens. Neuroreport, 2006, 17, 1629-1632.

[687] Bossong, M.G.; van Berckel, B.N.; Boellaard, R.; Zuurman, L.; Schuit, R.C.; Windhorst, A.D.; van Gerven, J.M.; Ramsey, N.F.; Lammertsma, A.A.; Kahn, R.S. $\Delta^{9}$-tetrahydrocannabinol induces dopamine release in the human striatum. Neuropsychopharmacol., 2009, 34(3), 759-766.

[688] Miller, A.S.; Walker, J.M. Effects of a cannabinoid on spontaneous and evoked neuronal activity in the substantia nigra pars reticulata. Eur. J .Pharmacol., 1995, 279(2-3), 179-185.

[689] Szabo, B.; Siemes, S., Wallmichrath, I. Inhibition of GABAergic neurotransmission in the ventral tegmental area by cannabinoids. Eur. J. Neurosci., 2002, 15(12), 2057-2061.

[690] Barrot, M.; Sesack, S.R.; Georges, F.; Pistis, M.; Hong, S.; Jhou, T.C. Braking dopamine systems: a new GABA master structure for mesolimbic and nigrostriatal functions. J. Neurosci., 2012, 32(41), 14094-14101.

[691] Julian, M.D.; Martin, A.B.; Cuellar, B.; Rodríguez De Fonseca, F.; Navarro, M.; Moratalla, R.; Garcia-Segura, L.M. Neuroanatomical relationship between type 1 cannabinoid receptors and dopaminergic systems in the rat basal ganglia. Neuroscience, 2003, 119, 309-318.

[692] Szabo, B.; Muller, T.; Koch, H. Effects of cannabinoids on dopamine release in the corpus striatum and the nucleus accumbens in vitro. $J$. Neurochem., 1999, 73, 1084-1089.

[693] Sidló, Z.; Reggio, P.H.; Rice, M.E. Inhibition of striatal dopamine release by $\mathrm{CB}_{1}$ receptor activation requires nonsynaptic communication involving GABA, $\mathrm{H}_{2} \mathrm{O}_{2}$, and $\mathrm{K}_{\text {ATP }}$ channels. Neurochem. Int., 2008, 52, 80-88.

[694] O'Neill, C.; Evers-Donnelly, A.; Nicholson, D.; O'Boyle, K.M.; O'Connor, J.J. D2 receptor-mediated inhibition of dopamine release in the rat striatum in vitro is modulated by CB1 receptors: studies using fast cyclic voltammetry. J. Neurochem., 2009, 108, 545-551.

[695] Cadogan, A.K.; Alexander, S.P.; Boyd, E.A.; Kendall, D.A. Influence of cannabinoids on electrically evoked dopamine release and cyclic AMP generation in the rat striatum. J. Neurochem., 1997, 69(3), 1131-1137.
[696] Sperlágh, B.; Windisch, K.; Andó, R.D.; Vizi, E.S. Neurochemical evidence that stimulation of $\mathrm{CB}_{1}$ cannabinoid receptors on GABAergic nerve terminals activates the dopaminergic reward system by increasing dopamine release in the rat nucleus accumbens. Neurochem. Int., 2009, 54, 452-457.

[697] Laviolette, S.R.; Grace, A.A. The roles of cannabinoid and dopamine receptor systems in neural emotional learning circuits: implications for schizophrenia and addiction. Cell Mol. Life Sci., 2006, 63(14), 1597-1613.

[698] Covey, D.P.; Wenzel, J.M.; Cheer, J.F. Cannabinoid modulation of drug reward and the implications of marijuana legalization. Brain Res., 2015, 1628(Pt A), 233-243.

[699] Wenzel, J.M.; Cheer, J.F. Endocannabinoid-dependent modulation of phasic dopamine signaling encodes external and internal rewardpredictive cues. Front. Psychiatry, 2014, 5, 118.

[700] Vinod, K.Y.; Yalamanchili, R.; Thanos, P.K.; Vadasz, C.; Cooper, T.B.; Volkow, N.D.; Hungund, B.L. Genetic and pharmacological manipulations of the $\mathrm{CB}_{1}$ receptor alter ethanol preference and dependence in ethanol preferring and nonpreferring mice. Synapse, 2008, 62(8), 574-581.

[701] Maldonado, R.; Robledo, P.; Berrendero, F. Endocannabinoid system and drug addiction: new insights from mutant mice approaches. Curr. Opin. Neurobiol., 2013, 23(4), 480-486.

[702] De Vries, T.J.; Shaham, Y.; Homberg, J.R.; Crombag, H.; Schuurman, K.; Dieben, J.; Vanderschuren, L.J.; Schoffelmeer, A.N. A cannabinoid mechanism in relapse to cocaine seeking. Nat. Med., 2001, 7(10), 1151-1154.

[703] Fattore, L.; Spano, M.S.; Deiana, S.; Melis, V.; Cossu, G.; Fadda, P.; Fratta, W. An endocannabinoid mechanism in relapse to drug seeking: a review of animal studies and clinical perspectives. Brain Res. Rev., 2007, 53, 1-16.

[704] Alvarez-Jaimes, L.; Polis, I.; Parsons, L.H. Attenuation of cueinduced heroin-seeking behavior by cannabinoid $\mathrm{CB}_{1}$ antagonist infusions into the nucleus accumbens core and prefrontal cortex, but not basolateral amygdala. Neuropsychopharmacol., 2008, 33, 24832493.

[705] Beardsley, P.M.; Thomas, B.F.; Mcmahon, L.R. Cannabinoid CB receptor antagonists as potential pharmacotherapies for drug abuse disorders. Int. Rev. Psychiatr., 2009, 21(2), 134-142.

[706] Ward, S.J.; Rosenberg, M.; Dykstra, L.A.; Walke,r E.A. The CB $_{1}$ antagonist rimonabant (SR141716) blocks cue-induced reinstatement of cocaine seeking and other context and extinction phenomena predictive of relapse. Drug Alcohol Depend., 2009, 105, 248-255.

[707] Cahill, K.; Ussher, M.H. Cannabinoid type 1 receptor antagonists for smoking cessation. Cochrane Database Syst. Rev., 2011, 3, CD005353.

[708] Jing, L.; Qiu, Y.; Zhang, Y.; Li, J.X. Effects of the cannabinoid CB 1 receptor allosteric modulator ORG 27569 on reinstatement of cocaine- and methamphetamine-seeking behavior in rats. Drug Alcohol Depend., 2014, 143, 251-256.

[709] Yang, P.; Wang, L.; Xie, X.Q. Latest advances in novel cannabinoid $\mathrm{CB}_{2}$ ligands for drug abuse and their therapeutic potential. Future Med. Chem., 2012, 4(2), 187-204.

[710] Callén, L.; Moreno, E.; Barroso-Chinea, P.; Moreno-Delgado, D.; Cortés, A.; Mallol, J.; Casadó, V.; Lanciego, J.L.; Franco, R.; Lluis, C.; Canela, E.I.; McCormick, P.J. Cannabinoid receptors $\mathrm{CB}_{1}$ and $\mathrm{CB}_{2}$ form functional heteromers in brain. J. Biol. Chem., 2012, 287, 20851-20865.

[711] Fergusson, D.M.; Horwood, L.J. Does cannabis use encourage other forms of illicit drug use? Addiction, 2000, 95(4), 505-520.

[712] Hall, W.D.; Lynskey, M. Is cannabis a gateway drug? Testing hypotheses about the relationship between cannabis use and the use of other illicit drugs. Drug Alcohol Rev., 2005, 24(1), 39-48.

[713] Mayet, A.; Legleye, S.; Beck, F.; Falissard, B.; Chau, N. The gateway hypothesis, common liability to addictions or the route of administration model a modelling process linking the three theories. Eur. Addict. Res., 2016, 22(2), 107-117.

[714] van de Giessen, E.; Weinstein, J.J.; Cassidy, C.M.; Haney, M.; Dong, Z.; Ghazzaoui, R.; Ojeil, N.; Kegeles, L.S.; Xu, X.; Vadhan, N.P.; Volkow, N.D.; Slifstein, M.; Abi-Dargham, A. Deficits in striatal 
dopamine release in cannabis dependence. Mol. Psychiatry, 2016, in press, doi:10.1038/mp.2016.21.

[715] Giuffrida, A.; Parsons, L.H.; Kerr, T.M.; Rodríguez de Fonseca, F.; Navarro, M.; Piomelli, D. Dopamine activation of endogenous cannabinoid signaling in dorsal striatum. Nat. Neurosci., 1999, 2(4), 358-363.

[716] Kreitzer, A.C.; Malenka, R.C. Striatal plasticity and basal ganglia circuit function. Neuron, 2008, 60(4), 543-554.

[717] Steffens, M.; Feuerstein, T.J. Receptor-independent depression of DA and 5-HT uptake by cannabinoids in rat neocortex--involvement of $\mathrm{Na}^{+} / \mathrm{K}^{+}$-ATPase. Neurochem. Int., 2004, 44, 529-538.

[718] Price, D.A.; Owens, W.A.; Gould, G.G.; Frazer, A.; Roberts, J.L.; Daws, L.C.; Giuffrida, A. CB1-independent inhibition of dopamine transporter activity by cannabinoids in mouse dorsal striatum. $J$. Neurochem., 2007, 101, 389-396.

[719] Pandolfo, P.; Silveirinha, V.; dos Santos-Rodrigues, A.; Venance, L.; Ledent, C.; Takahashi, R.N.; Cunha, R.A.; Köfalvi, A. Cannabinoids inhibit the synaptic uptake of adenosine and dopamine in the rat and mouse striatum. Eur. J .Pharmacol., 2011, 655(1-3), 38-45.

[720] Soreq, H. Checks and balances on cholinergic signaling in brain and body function. Trends Neurosci., 2015, 38(7), 448-458.

[721] Gifford, A.N.; Ashby, Jr C.R. Electrically evoked acetylcholine release from hippocampal slices is inhibited by the cannabinoid receptor agonist, WIN 55212-2, and is potentiated by the cannabinoid antagonist, SR141716A. J. Pharmacol. Exp. Ther., 1996, 277, 14311436.

[722] Nakazi, M.; Bauer, U.; Nickel, T.; Kathmann, M.; Schlicker, E. Inhibition of serotonin release in the mouse brain via presynaptic cannabinoid $\mathrm{CB}_{1}$ receptors. Naunyn. Schmiedebergs Arch. Pharmacol., 2000, 361(1), 19-24.

[723] Kathmann, M.; Weber, B.; Schlicker, E. Cannabinoid CB1 receptormediated inhibition of acetylcholine release in the brain of NMRI, CD-1 and C57BL/6J mice. Naunyn. Schmiedebergs Arch. Pharmacol., 2001, 363, 50-56.

[724] Steffens, M.; Szabo, B.; Klar, M.; Rominger, A.; Zentner, J.; Feuerstein, T.J. Modulation of electrically evoked acetylcholine release through cannabinoid CB1 receptors: evidence for an endocannabinoid tone in the human neocortex. Neuroscience, 2003, 120(2), 455-465

[725] Gessa, G.L.; Mascia, M.S.; Casu, M.A.; Carta, G. Inhibition of hippocampal acetylcholine release by cannabinoids: reversal by SR 141716A. Eur. J. Pharmacol., 1997, 327, R1-R2.

[726] Gessa, G.L.; Casu, M.A.; Carta, G.; Mascia, M.S. Cannabinoids decrease acetylcholine release in the medial-prefrontal cortex and hippocampus; reversal by SR 141716A. Eur. J .Pharmacol., 1998, 355(2-3), 119-124.

[727] Tzavara, E.T.; Wade, M.; Nomikos, G.G. Biphasic effects of cannabinoids on acetylcholine release in the hippocampus: site and mechanism of action. J. Neurosci., 2003a, 23(28), 9374-9384.

[728] Nyíri, G.; Szabadits, E.; Cserép, C.; Mackie, K.; Shigemoto, R.; Freund, T.F. $\mathrm{GABA}_{\mathrm{B}}$ and $\mathrm{CB}_{1}$ cannabinoid receptor expression identifies two types of septal cholinergic neurons. Eur. J. Neurosci., 2005, 21(11), 3034-3042.

[729] Keimpema, E.; Alpár, A.; Howell, F.; Malenczyk, K.; Hobbs, C.; Hurd, Y.L.; Watanabe, M.; Sakimura, K.; Kano, M.; Doherty, P.; Harkany, T. Diacylglycerol lipase $\alpha$ manipulation reveals developmental roles for intercellular endocannabinoid signaling. Sci. Rep., 2013, 3, 2093.

[730] Harkany, T.; Härtig, W.; Berghuis, P.; Dobszay, M.B.; Zilberter, Y.; Edwards, R.H.; Mackie, K.; Ernfors, P. Complementary distribution of type 1 cannabinoid receptors and vesicular glutamate transporter 3 in basal forebrain suggests input-specific retrograde signalling by cholinergic neurons. Eur. J. Neurosci., 2003, 18(7), 1979-1992.

[731] Harkany, T.; Dobszay, M.B.; Cayetanot, F.; Härtig, W.; Siegemund, T.; Aujard, F.; Mackie, K. Redistribution of $\mathrm{CB}_{1}$ cannabinoid receptors during evolution of cholinergic basal forebrain territories and their cortical projection areas: a comparison between the gray mouse lemur (Microcebus murinus, primates) and rat. Neuroscience, 2005, 135(2), 595-609.
[732] Hohmann, A.G.; Herkenham, M. Localization of cannabinoid $\mathrm{CB}_{\mathrm{s}}$ receptor mRNA in neuronal subpopulations of rat striatum: A doublelabel in situ hybridization study. Synapse, 2000, 37, 71-80.

[733] Narushima, M.; Uchigashima, M.; Fukaya, M.; Matsui, M.; Manabe, T.; Hashimoto, K.; Watanabe, M.; Kano, M. Tonic enhancement of endocannabinoid-mediated retrograde suppression of inhibition by cholinergic interneuron activity in the striatum. J. Neurosci., 2007, 27(3), 496-506.

[734] Alger, B.E.; Nagode, D.A.; Tang, A.H. Muscarinic cholinergic receptors modulate inhibitory synaptic rhythms in hippocampus and neocortex. Front. Synaptic Neurosci., 2014, 6, 18.

[735] Barann, M.; Molderings, G.; Brüss, M.; Bönisch, H.; Urban, B.W.; Göthert, M. Direct inhibition by cannabinoids of human $5-\mathrm{HT}_{3 \mathrm{~A}}$ receptors: probable involvement of an allosteric modulatory site. $\mathrm{Br}$. J. Pharmacol., 2002, 137(5), 589-596.

[736] Oz, M.; Al Kury, L.; Keun-Hang, S.Y.; Mahgoub, M.; Galadari, S. Cellular approaches to the interaction between cannabinoid receptor ligands and nicotinic acetylcholine receptors. Eur. J. Pharmacol., 2014, 731, 100-105.

[737] Mahgoub, M.; Keun-Hang, S.Y.; Sydorenko, V.; Ashoor, A.; Kabbani, N.; Al Kury, L.; Sadek, B.; Howarth, C.F.; Isaev, D.; Galadari, S.; Oz, M. Effects of cannabidiol on the function of $\alpha 7$ nicotinic acetylcholine receptors. Eur. J. Pharmacol., 2013, 720(1-3), 310-319.

[738] Justinová, Z.; Mascia, P.; Wu, H.Q.; Secci, M.E.; Redhi, G.H.; Panlilio, L.V.; Scherma, M.; Barnes, C.; Parashos, A.; Zara, T. Fratta, W.; Solinas, M.; Pistis, M.; Bergman, J.; Kangas, B.D.; Ferré, S.; Tanda, G.; Schwarcz, R.; Goldberg, S.R. Reducing cannabinoid abuse and preventing relapse by enhancing endogenous brain levels of kynurenic acid. Nat. Neurosci., 2013, 16(11), 1652-1661.

[739] Adler, L.E.; Olincy, A.; Waldo, M.; Harris, J.G.; Griffith, J.; Stevens, K.; Flach, K.; Nagamoto, H.; Bickford, P.; Leonard, S.; Freedman, R. Schizophrenia, sensory gating, and nicotinic receptors. Schizophr. Bull., 1998, 24, 189-202.

[740] Olincy, A.; Johnson, L.L.; Ross, R.G. Differential effects of cigarette smoking on performance of a smooth pursuit and a saccadic eye movement task in schizophrenia. Psychiatry Res., 2003, 117, 223236.

[741] Olincy, A.; Harris, J.G.; Johnson, L.L.; Pender, V.; Kongs, S.; Allensworth, D.; Ellis, J.; Zerbe, G.O.; Leonard, S.; Stevens, K.E.; Stevens, J.O.; Martin, L.; Adler, L.E.; Soti, F.; Kem, W.R.; Freedman, R. Proof-of-concept trial of an alpha7 nicotinic agonist in schizophrenia. Arch. Gen. Psychiatry, 2006, 63, 630-638.

[742] Pichat, P.; Bergis, O.E.; Terranova, J.P.; Urani, A.; Duarte, C.; Santucci, V.; Gueudet, C.; Voltz, C.; Steinberg, R.; Stemmelin, J.; Oury-Donat, F.; Avenet, P.; Griebel, G.; Scatton, B. SSR180711, a novel selective alpha7 nicotinic receptor partial agonist: (II) efficacy in experimental models predictive of activity against cognitive symptoms of schizophrenia. Neuropsychopharmacol., 2007, 32, 1734.

[743] Stella, N.; Piomelli, D. Receptor-dependent formation of endogenous cannabinoids in cortical neurons. Eur. J. Pharmacol., 2001, 425(3), 189-196.

[744] Tzavara, E.T.; Davis, R.J.; Perry, K.W.; Li, X.; Salhoff, C.; Bymaster, F.P.; Witkin, J.M.; Nomikos, G.G. The $\mathrm{CB}_{1}$ receptor antagonist SR141716A selectively increases monoaminergic neurotransmission in the medial prefrontal cortex: implications for therapeutic actions. Br. J .Pharmacol., 2003, 138(4), 544-553.

[745] Ortega, J.E.; Gonzalez-Lira, V.; Horrillo, I.; Herrera-Marschitz, M.; Callado, L.F.; Meana, J.J. Additive effect of rimonabant and citalopram on extracellular serotonin levels monitored with in vivo microdialysis in rat brain. Eur. J. Pharmacol., 2013, 709(1-3), 13-19.

[746] Tao, R.; Ma, Z. Neural circuit in the dorsal raphe nucleus responsible for cannabinoid-mediated increases in 5-HT efflux in the nucleus accumbens of the rat brain. ISRN Pharmacol., 2012, 2012, 276902.

[747] Häring, M.; Marsicano, G.; Lutz, B.; Monory, K. Identification of the cannabinoid receptor type 1 in serotonergic cells of raphe nuclei in mice. Neuroscience, 2007, 146(3), 1212-1219.

[748] Oropeza, V.C.; Mackie, K.; van Bockstaele, E.J. Cannabinoid receptors are localized to noradrenergic axon terminals in the rat frontal cortex. Brain Res., 2007, 1127(1), 36-44. 
[749] Richter, H.; Teixeira, F.M.; Ferreira, S.G.; Kittel, Á.; Köfalvi, A.; Sperlágh, B. Presynaptic $\alpha 2$-adrenoceptors control the inhibitory action of presynaptic $\mathrm{CB}_{1}$ cannabinoid receptors on prefrontocortical norepinephrine release in the rat. Neuropharmacology, 2012, 63(5), 784-797.

[750] Reyes, B.A.; Heldt, N.A.; Mackie, K.; van Bockstaele, E.J. Ultrastructural evidence for synaptic contacts between cortical noradrenergic afferents and endocannabinoid-synthesizing postsynaptic neurons. Neuroscience, 2015, 303, 323-337.

[751] Schlicker, E.; Timm, J.; Zentner, J.; Göthert, M. Cannabinoid $\mathrm{CB}_{1}$ receptor-mediated inhibition of noradrenaline release in the human and guinea-pig hippocampus. Naunyn. Schmiedebergs Arch. Pharmacol., 1997, 356(5), 583-589.

[752] Balázsa, T.; Bíró, J.; Gullai, N.; Ledent, C.; Sperlágh, B. CB - $_{1-1}$ cannabinoid receptors are involved in the modulation of non-synaptic $\left[{ }^{3} \mathrm{H}\right]$ serotonin release from the rat hippocampus. Neurochem. Int., 2008, 52(1-2), 95-102.

[753] El-Alfy, A.T.; Ivey, K.; Robinson, K.; Ahmed, S.; Radwan, M.; Slade, D.; Khan, I.; ElSohly, M.A.; Ross, S. Antidepressant-like effect of $\Delta 9$-tetrahydrocannabinol and other cannabinoids isolated from Cannabis sativa L. Pharmacol. Biochem. Behav., 2010, 95(4), 434-442.

[754] Hill, M.N.; Sun, J.C.; Tse, M.T.; Gorzalka, B.B. Altered responsiveness of serotonin receptor subtypes following long-term cannabinoid treatment. Int J Neuropsychopharmacol, 2006, 9(3), 277-286.

[755] Sagredo, O.; Ramos, J.A.; Fernández-Ruiz, J.; Rodríguez, M.L.; de Miguel, R. Chronic $\Delta^{9}$-tetrahydrocannabinol administration affects serotonin levels in the rat frontal cortex. Naunyn. Schmiedebergs Arch. Pharmacol., 2006, 372(4), 313-317.

[756] Franklin, J.M.; Carrasco, G.A. G-protein receptor kinase 5 regulates the cannabinoid receptor 2-induced up-regulation of serotonin 2A receptors. J. Biol. Chem., 2013, 288(22), 15712-15724.

[757] Franklin, J.M.; Vasiljevik, T.; Prisinzano, T.E.; Carrasco, G.A. Cannabinoid agonists increase the interaction between $\beta$-arrestin 2 and ERK1/2 and upregulate $\beta$-arrestin 2 and 5- $\mathrm{HT}_{2 \mathrm{~A}}$ receptors. Pharmacol. Res., 2013, 68(1), 46-58.

[758] Braida, D.; Limonta, V.; Malabarba, L.; Zani, A.; Sala, M. 5-HT ${ }_{1 \mathrm{~A}}$ receptors are involved in the anxiolytic effect of $\Delta^{9}$ tetrahydrocannabinol and AM 404, the anandamide transport inhibitor, in Sprague-Dawley rats. Eur. J. Pharmacol., 2007, 555(23), 156-163.

[759] Bambico, F.R.; Hattan, P.R.; Garant, J.P.; Gobbi, G. Effect of delta9-tetrahydrocannabinol on behavioral despair and on pre- and postsynaptic serotonergic transmission. Prog. Neuropsychopharmacol. Biol. Psychiat., 2012, 38(1), 88-96.

[760] Häring, M.; Grieb, M.; Monory, K.; Lutz, B.; Moreira, F.A. Cannabinoid $\mathrm{CB}_{1}$ receptor in the modulation of stress coping behavior in mice: the role of serotonin and different forebrain neuronal subpopulations. Neuropharmacology, 2013, 65, 83-89.

[761] Häring, M.; Enk, V.; Aparisi Rey, A.; Loch, S.; Ruiz de Azua, I.; Weber, T.; Bartsch, D.; Monory, K.; Lutz, B. Cannabinoid type-1 receptor signaling in central serotonergic neurons regulates anxietylike behavior and sociability. Front. Behav. Neurosci., 2015, 9, 235.

[762] McLaughlin, R.J.; Hill, M.N.; Bambico, F.R.; Stuhr, K.L.; Gobbi, G.; Hillard, C.J.; Gorzalka, B.B. Prefrontal cortical anandamide signaling coordinates coping responses to stress through a serotonergic pathway. Eur. Neuropsychopharmacol., 2012, 22(9), 664-671.

[763] Bolognini, D.; Rock, E.M.; Cluny, N.L.; Cascio, M.G.; Limebeer, C.L.; Duncan, M.; Stott, C.G.; Javid, F.A.; Parker, L.A.; Pertwee, R.G. Cannabidiolic acid prevents vomiting in Suncus murinus and nausea-induced behaviour in rats by enhancing $5-\mathrm{HT}_{1 \mathrm{~A}}$ receptor activation. Br. J. Pharmacol., 2013, 168(6), 1456-1470.

[764] Cascio, M.G.; Zamberletti, E.; Marini, P.; Parolaro, D.; Pertwee, R.G. The phytocannabinoid, $\Delta^{9}$-tetrahydrocannabivarin, can act through 5$\mathrm{HT}_{1 \mathrm{~A}}$ receptors to produce antipsychotic effects. Br. J. Pharmacol., 2015, 172(5), 1305-1318.

[765] Russo, E.B.; Burnett, A.; Hall, B.; Parker, K.K. Agonistic properties of cannabidiol at 5-HT $\mathrm{Ha}_{1 \mathrm{a}}$ receptors. Neurochem. Res., 2005, 30, 10371043.
[766] Campos, A.C.; Guimaraes, F.S. Involvement of $5 \mathrm{HT}_{1 \mathrm{~A}}$ receptors in the anxiolytic-like effects of cannabidiol injected into the dorsolateral periaqueductal gray of rats. Psychopharmacology (Berl), 2008, 199, 223-230.

[767] Rock, E.M.; Bolognini, D.; Limebeer, C.L.; Cascio, M.G.; AnaviGoffer, S.; Fletcher, P.J.; Mechoulam, R.; Pertwee, R.G.; Parker, L.A. Cannabidiol, a non-psychotropic component of cannabis, attenuates vomiting and nausea-like behaviour via indirect agonism of 5- $\mathrm{HT}_{1 \mathrm{~A}}$ somatodendritic autoreceptors in the dorsal raphe nucleus. $\mathrm{Br}$. $J$ .Pharmacol., 2012, 165(8), 2620-2634.

[768] Gomes, F.V.; Del Bel, E.A.; Guimarães, F.S. Cannabidiol attenuates catalepsy induced by distinct pharmacological mechanisms via 5$\mathrm{HT}_{1 \mathrm{~A}}$ receptor activation in mice. Prog. Neuropsychopharmacol. Biol. Psychiatry, 2013, 46, 43-47.

[769] Ward, S.J.; McAllister, S.D.; Kawamura, R.; Murase, R.; Neelakantan, H.; Walker, E.A. Cannabidiol inhibits paclitaxelinduced neuropathic pain through 5-HT(1A) receptors without diminishing nervous system function or chemotherapy efficacy. $B r . J$. Pharmacol., 2014, 171(3), 636-645.

[770] Zanelati, T.V.; Biojone, C.; Moreira, F.A.; Guimaraes, F.S.; Joca, S.R. Antidepressant-like effects of cannabidiol in mice: possible involvement of 5- $\mathrm{HT}_{1 \mathrm{~A}}$ receptors. Br. J. Pharmacol., 2010, 159, 122128.

[771] Mishima, K.; Hayakawa, K.; Abe, K.; Ikeda, T.; Egashira, N.; Iwasaki, K.; Fujiwara, M. Cannabidiol prevents cerebral infarction via a serotonergic 5-hydroxytryptamine1A receptor-dependent mechanism. Stroke, 2005, 36(5), 1077-1082.

[772] Hayakawa, K.; Mishima, K.; Nozako, M.; Ogata, A.; Hazekawa, M.; Liu, A.X.; Fujioka, M.; Abe, K.; Hasebe, N.; Egashira, N.; Iwasaki, K.; Fujiwara, M. Repeated treatment with cannabidiol but not $\Delta^{9}$ tetrahydrocannabinol has a neuroprotective effect without the development of tolerance. Neuropharmacology, 2007, 52(4), 10791087.

[773] Hayakawa, K.; Mishima, K.; Irie, K.; Hazekawa, M.; Mishima, S.; Fujioka, M.; Orito, K.; Egashira, N.; Katsurabayashi, S.; Takasaki, K.; Iwasaki, K.; Fujiwara, M. Cannabidiol prevents a post-ischemic injury progressively induced by cerebral ischemia via a high-mobility group box1-inhibiting mechanism. Neuropharmacology, 2008, 55(8), 1280-1286.

[774] Parker, L.A.; Limebeer, C.L. In: Cannabinoids and the Brain; Köfalvi, A., Ed. Springer US, 2008, pp. 259-276.

[775] Hill, K.P. Medical marijuana: more questions than answers. $J$. Psychiatr. Pract., 2014, 20(5), 389-391.

[776] Todaro, B. Cannabinoids in the treatment of chemotherapy-induced nausea and vomiting. J. Natl. Compr. Canc. Netw., 2012, 10(4), 487492.

[777] van Sickle, M.D.; Duncan, M.; Kingsley, P.J.; Mouihate, A.; Urbani, P.; Mackie, K.; Stella, N.; Makriyannis, A.; Piomelli, D.; Davison, J.S.; Marnett, L.J.; Di Marzo, V.; Pittman, Q.J.; Patel, K.D.; Sharkey, K.A. Identification and functional characterization of brainstem cannabinoid CB2 receptors. Science, 2005, 310, 329-332.

[778] Parker, L.A.; Rock, E.M.; Limebeer, C.L. Regulation of nausea and vomiting by cannabinoids. Br. J. Pharmacol., 2011, 163(7), 14111422.

[779] Patterson, D.A.; Smith, E.; Monahan, M.; Medvecz, A.; Hagerty, B.; Krijger, L.; Chauhan, A.; Walsh, M. Cannabinoid hyperemesis and compulsive bathing: a case series and paradoxical pathophysiological explanation. J. Am. Board Fam. Med., 2010, 23(6), 790-793.

[780] Beech, R.A.; Sterrett, D.R.; Babiuk, J.; Fung, H. Cannabinoid hyperemesis syndrome: A case report and literature review. J. Oral Maxillofac. Surg., 2015, 73(10), 1907-1910.

[781] Habboushe, J.; Sedor, J. Cannabinoid hyperemesis acute renal failure: a common sequela of cannabinoid hyperemesis syndrome. Am. J. Emerg. Med., 2014, 32(6), 690.e1-2.

[782] Cheer, J.F.; Cadogan, A.K.; Marsden, C.A.; Fone, K.C.; Kendall, D.A. Modification of 5- $\mathrm{HT}_{2}$ receptor mediated behaviour in the rat by oleamide and the role of cannabinoid receptors. Neuropharmacology, 1999, 38(4), 533-541.

[783] Devlin, M.G.; Christopoulos, A. Modulation of cannabinoid agonist binding by $5-\mathrm{HT}$ in the rat cerebellum. J. Neurochem., 2002, 80(6), 1095-1102. 
[784] Viñals, X.; Moreno, E.; Lanfumey, L.; Cordomí, A.; Pastor, A.; de La Torre, R.; Gasperini, P.; Navarro, G.; Howell, L.A.; Pardo, L.; Lluís, C.; Canela, E.I.; McCormick, P.J.; Maldonado, R.; Robledo, P. Cognitive impairment induced by $\Delta^{9}$-tetrahydrocannabinol occurs through heteromers between cannabinoid $\mathrm{CB}_{1}$ and serotonin $5-\mathrm{HT}_{2 \mathrm{~A}}$ receptors. PLoS Biol., 2015, 13(7), e1002194.

[785] Wang, H.; Treadway, T.; Covey, D.P.; Cheer, J.F.; Lupica, C.R. Cocaine-Induced Endocannabinoid Mobilization in the Ventral Tegmental Area. Cell Rep., 2015, 12(12), 1997-2008.

[786] Haj-Dahmane, S.; Shen, R.Y. Chronic stress impairs $\alpha_{1}$-adrenoceptorinduced endocannabinoid-dependent synaptic plasticity in the dorsal raphe nucleus. J. Neurosci., 2014, 34(44), 14560-14570.

[787] Best, A.R.; Regehr, W.G. Serotonin evokes endocannabinoid release and retrogradely suppresses excitatory synapses. J. Neurosci., 2008, 28(25), 6508-6515.

[788] Cascio, M.G.; Gauson, L.A.; Stevenson, L.A.; Ross, R.A.; Pertwee, R.G. Evidence that the plant cannabinoid cannabigerol is a highly potent $\alpha 2$-adrenoceptor agonist and moderately potent $5 \mathrm{HT}_{1 \mathrm{~A}}$ receptor antagonist. Br. J. Pharmacol., 2010, 159(1), 129-141.

[789] Cathel, A.M.; Reyes, B.A.; Wang, Q.; Palma, J.; Mackie, K.; van Bockstaele, E.J.; Kirby, L.G. Cannabinoid modulation of alpha adrenergic receptor function in rodent medial prefrontal cortex. Eur. J. Neurosci., 2014, 40(8), 3202-3214.

[790] Margulies, J.E.; Hammer, R.P. $\Delta^{9}$-tetrahydrocannabinol alter cerebral metabolism in a biphasic, dose-dependent manner in rat brain. Eur. J. Pharmacol., 1991, 202, 373-378.

[791] Turkanis, S.A.; Karler, R. Excitatory and depressant effects of delta 9-tetrahydrocannabinol and cannabidiol on cortical evoked responses in the conscious rat. Psychopharmacology (Berl.), 1981, 75, 294-298.

[792] Metna-Laurent, M.; Soria-Gómez, E.; Verrier, D.; Conforzi, M.; Jégo, P.; Lafenêtre, P.; Marsicano, G. Bimodal control of fear-coping strategies by $\mathrm{CB}_{1}$ cannabinoid receptors. J. Neurosci., 2012, 32(21), 7109-7118.

[793] Sales-Carbonell, C.; Rueda-Orozco, P.E.; Soria-Gómez, E.; Buzsáki, G.; Marsicano, G.; Robbe, D. Striatal GABAergic and cortical glutamatergic neurons mediate contrasting effects of cannabinoids on cortical network synchrony. Proc. Natl. Acad. Sci. USA, 2013, 110(2), 719-724.

[794] Piet, R.; Garenne, A.; Farrugia, F.; Le Masson, G.; Marsicano, G.; Chavis, P.; Manzoni, O.J. State-dependent, bidirectional modulation of neural network activity by endocannabinoids. J. Neurosci., 2011, 31(46), 16591-16596.

[795] Steindel, F.; Lerner, R.; Häring, M.; Ruehle, S.; Marsicano, G.; Lutz, B.; Monory, K. Neuron-type specific cannabinoid-mediated G protein signalling in mouse hippocampus. J. Neurochem., 2013, 124(6), 795807.

[796] Ademark, L.; Lovinger, D.M. Frequency-dependent inversion of net striatal output by endocannabinoid-dependent plasticity at different synaptic inputs. J. Neurosci., 2009, 29(5), 1375-1380.

[797] Ferré, S.; Baler, R.; Bouvier, M.; Caron, M.G.; Devi, L.A.; Durroux, T.; Fuxe, K.; George, S.R.; Javitch, J.A.; Lohse, M.J.; Mackie, K.; Milligan, G.; Pfleger, K.D.; Pin, J.P.; Volkow, N.D.; Waldhoer, M.; Woods, A.S.; Franco, R. Building a new conceptual framework for receptor heteromers. Nat. Chem. Biol., 2009, 5(3) 131-134.

[798] Ferré, S.; Agnati, L.F.; Ciruela, F.; Lluis, C.; Woods, A.S.; Fuxe, K.; Franco, R. Neurotransmitter receptor heteromers and their integrative role in 'local modules': the striatal spine module. Brain Res. Rev., 2007, 55(1), 55-67.

[799] Fuxe, K.; Marcellino, D.; Guidolin, D.; Woods, A.S.; Agnati, L.F. Heterodimers and receptor mosaics of different types of G-proteincoupled receptors. Physiology (Bethesda), 2008, 23, 322-332.

[800] Fuxe, K.; Marcellino, D.; Woods, A.S.; Giuseppina, L.; Antonelli, T.; Ferraro, L.; Tanganelli, S.; Agnati, L.F. Agnati Integrated signaling in heterodimers and receptor mosaics of different types of GPCRs of the forebrain: relevance for schizophrenia. J. Neural. Transm., 2009, 116(8), 923-939.

[801] Cabello, N.; Gandía, J.; Bertarelli, D.C.; Watanabe, M.; Lluís, C.; Franco, R.; Ferré, S.; Luján, R.; Ciruela, F. Metabotropic glutamate type 5 , dopamine $D_{2}$ and adenosine $A_{2 a}$ receptors form higher-order oligomers in living cells. J. Neurochem., 2009, 109(5), 1497-1507.
[802] Ciruela, F.; Fernández-Dueñas, V.; Llorente, J.; Borroto-Escuela, D.; Cuffí, M.L.; Carbonell, L.; Sánchez, S.; Agnati, L.F.; Fuxe, K.; Tasca, C.I. G protein-coupled receptor oligomerization and brain integration: focus on adenosinergic transmission. Brain Res., 2012 1476, 86-95.

[803] Rozenfeld, R.; Bushlin, I.; Gomes, I.; Tzavaras, N.; Gupta, A.; Neves, S.; Battini, L.; Gusella, G.L.; Lachmann, A.; Ma'ayan, A.; Blitzer, R.D.; Devi, L.A. Receptor heteromerization expands the repertoire of cannabinoid signaling in rodent neurons. PLOS ONE, 2012, 7(1), e29239.

[804] Brugarolas, M.; Navarro, G.; Martínez-Pinilla, E.; Angelats, E.; Casadó, V.; Lanciego, J.L.; Franco, R. G-protein-coupled receptor heteromers as key players in the molecular architecture of the central nervous system. CNS Neurosci. Ther., 2014, 20(8), 703-709.

[805] Navarro, G.; Ferré, S.; Cordomi, A.; Moreno, E.; Mallol, J.; Casadó, V.; Cortés, A.; Hoffmann, H.; Ortiz, J.; Canela, E.I.; Lluís, C.; Pardo, L.; Franco, R.; Woods, A.S. Interactions between intracellular domains as key determinants of the quaternary structure and function of receptor heteromers. J. Biol. Chem., 2010, 285(35), 27346-27359.

[806] Bonaventura, J.; Navarro, G.; Casadó-Anguera, V.; Azdad, K.; Rea, W.; Moreno, E.; Brugarolas, M.; Mallol, J.; Canela, E.I.; Lluís, C.; Cortés, A.; Volkow, N.D.; Schiffmann, S.N.; Ferré, S.; Casadó, V. Allosteric interactions between agonists and antagonists within the adenosine $\mathrm{A}_{2 \mathrm{~A}}$ receptor-dopamine $\mathrm{D}_{2}$ receptor heterotetramer. Proc. Natl. Acad. Sci. USA, 2015, 112(27), E3609-3618.

[807] Katona, I.; Rancz, E.A.; Acsady, L.; Ledent, C.; Mackie, K.; Hajos, N.; Freund, T.F. Distribution of $\mathrm{CB}_{1}$ cannabinoid receptors in the amygdala and their role in the control of GABAergic transmission. $J$. Neurosci., 2001, 21, 9506-9518.

[808] Wager-Miller, J.; Westenbroek, R.; Mackie, K. Dimerization of G protein-coupled receptors: $\mathrm{CB}_{1}$ cannabinoid receptors as an example. Chem. Phys. Lipids, 2002, 121, 83-89.

[809] Sierra, S.; Luquin, N.; Rico, A.J.; Gómez-Bautista, V.; Roda, E.; Dopeso-Reyes, I.G.; Vázquez, A.; Martínez-Pinilla, E.; LabandeiraGarcía, J.L.; Franco, R.; Lanciego, J.L. Detection of cannabinoid receptors $\mathrm{CB}_{1}$ and $\mathrm{CB}_{2}$ within basal ganglia output neurons in macaques: changes following experimental parkinsonism. Brain Struct Funct., 2015, 220(5), 2721-2738.

[810] Balenga, N.A.,; Martínez-Pinilla, E.; Kargl, J.; Schröder, R.; Peinhaupt, M.; Platzer, W.; Bálint, Z.; Zamarbide, M.; DopesoReyes, I.G.; Ricobaraza, A.; Pérez-Ortiz, J.M.; Kostenis, E.; Waldhoer, M.; Heinemann, A.; Franco, R. Heteromerization of GPR55 and cannabinoid $\mathrm{CB}_{2}$ receptors modulates signalling. Br. $J$. Pharmacol., 2014, 171(23), 5387-5406.

[811] Moreno, E.; Andradas, C.; Medrano, M.; Caffarel, M.M.; PérezGómez, E.; Blasco-Benito, S.; Gómez-Cañas, M.; Pazo,s M.R.; Irving, A.J.; Lluís, C.; Canela, E.I.; Fernández-Ruiz, J.; Guzmán, M.; McCormick, P.J.; Sánchez, C. Targeting CB $_{2}$-GPR55 receptor heteromers modulates cancer cell signaling. J. Biol. Chem., 2014, 289(32), 21960-20972.

[812] Martínez-Pinilla, E.; Reyes-Resina, I.; Oñatibia-Astibia, A.; Zamarbide, M.; Ricobaraza, A.; Navarro, G.; Moreno, E.; DopesoReyes, I.G.; Sierra, S.; Rico, A.J.; Roda, E.; Lanciego, J.L.; Franco, R. $\mathrm{CB}_{1}$ and GPR55 receptors are co-expressed and form heteromers in rat and monkey striatum. Exp. Neurol., 2014, 261, 44-52.

[813] Rozenfeld, R.; Gupta, A.; Gagnidze, K.; Lim, M.P.; Gomes, I.; LeeRamos, D.; Nieto, N.; Devi, L.A. $\mathrm{AT}_{1} \mathrm{R}-\mathrm{CB}_{1} \mathrm{R}$ heteromerization reveals a new mechanism for the pathogenic properties of angiotensin II. EMBO J., 2011, 30, 2350-2363.

[814] Ellis, J.; Pediani, J.D.; Canals, M.; Milasta, S.; Milligan, G. Orexin-1 receptor-cannabinoid $\mathrm{CB}_{1}$ receptor heterodimerization results in both ligand-dependent and -independent coordinated alterations of receptor localization and function. J. Biol. Chem., 2006, 281(50), 38812-38824.

[815] Ward, R.J.; Pediani, J.D.; Milligan, G. Heteromultimerization of cannabinoid $\mathrm{CB}_{1}$ receptor and orexin $\mathrm{OX}_{1}$ receptor generates a unique complex in which both protomers are regulated by orexin A. J. Biol. Chem., 2011, 286, 37414-37428.

[816] Perrey, D.A.; Gilmour, B.P.; Thomas, B.F.; Zhang, Y. Toward the Development of Bivalent Ligand Probes of Cannabinoid $\mathrm{CB}_{1}$ and Orexin OX1 Receptor Heterodimers. ACS Med. Chem. Lett., 2014, 5(6), 634-638. 
[817] Glass, M.; Felder, C.C. Concurrent stimulation of cannabinoid $\mathrm{CB}_{1}$ and dopamine $\mathrm{D}_{2}$ receptors augments cAMP accumulation in striatal neurons: evidence for a $\mathrm{G}_{\mathrm{s}}$ linkage to the $\mathrm{CB}_{1}$ receptor. J. Neurosci., 1997, 17(14), 5327-5333.

[818] Jarrahian, A.; Watts, V.J.; Barker, E.L. $\mathrm{D}_{2}$ dopamine receptors modulate $\mathrm{G} \alpha$-subunit coupling of the $\mathrm{CB}_{1}$ cannabinoid receptor. $J$. Pharmacol. Exp. Ther., 2004, 308, 880-886.

[819] Kearn, C.S.; Blake-Palmer, K.; Daniel, E.; Mackie, K.; Glass, M. Concurrent stimulation of cannabinoid $\mathrm{CB}_{1}$ and dopamine $\mathrm{D}_{2}$ receptors enhances heterodimer formation: A mechanism for receptor cross-talk? Molecular Pharmacology, 2005, 67, 1697-1704.

[820] Khan, S.S.; Lee, F.J. Delineation of domains within the cannabinoid $\mathrm{CB}_{1}$ and dopamine $\mathrm{D}_{2}$ receptors that mediate the formation of the heterodimer complex. J. Mol. Neurosci., 2014, 53(1), 10-21.

[821] Pickel, V.M.; Chan, J.; Kearn, C.S.; Mackie, K. Targeting dopamine $\mathrm{D}_{2}$ and cannabinoid-1 $\left(\mathrm{CB}_{1}\right)$ receptors in rat nucleus accumbens. $J$. Comp. Neurol., 2006, 495(3), 299-313.

[822] Carriba, P.; Ortiz, O.; Patkar, K.; Justinova, Z.; Stroik, J.; Themann, A.; Müller, C.; Woods, A. S.; Hope, B. T.; Ciruela, F.; Casadó, V.; Canela, E. I.; Lluis, C.; Goldberg, S. R.; Moratalla, R.; Franco, R.; Ferré, S. Striatal adenosine $\mathrm{A}_{2 \mathrm{~A}}$ and cannabinoid $\mathrm{CB}_{1}$ receptors form functional heteromeric complexes that mediate the motor effects of cannabinoids. Neuropsychopharmacol., 2007, 32, 2249-2259.

[823] Ferré, S.; Lluís, C.; Justinova, Z.; Quiroz, C.; Orru, M.; Navarro, G.; Canela, E.I.; Franco, R.; Goldberg, S.R. Adenosine-cannabinoid receptor interactions. Implications for striatal function. $\mathrm{Br}$. J. Pharmacol., 2010, 160(3), 443-453.

[824] Ferré, S.; Quiroz, C.; Orru, M.; Guitart, X.; Navarro, G.; Cortés, A.; Casadó, V.; Canela, E.I.; Lluis, C.; Franco, R. Adenosine $\mathrm{A}_{2 \mathrm{~A}}$ receptors and $A_{2 A}$ receptor heteromers as key players in striatal function. Front. Neuroanat., 2011, 5, 36.

[825] Chiodi, V.; Ferrante, A.; Ferraro, L.; Potenza, R.L.; Armida, M.; Beggiato, S.; Pèzzola, A.; Bader, M.; Fuxe, K.; Popoli, P.; Domenici, M.R. Striatal adenosine-cannabinoid receptor interactions in rats overexpressing adenosine $\mathrm{A}_{2 \mathrm{~A}}$ receptors. J. Neurochem., 2015, 136(5), 907-917.

[826] Cunha, R.A. Different cellular sources and different roles of adenosine: $A_{1}$ receptor-mediated inhibition through astrocytic-driven volume transmission and synapse-restricted $\mathrm{A}_{2 \mathrm{~A}}$ receptor-mediated facilitation of plasticity. Neurochem. Int., 2008, 52(1-2), 65-72.

[827] Ciruela, F.; Ferré, S.; Casadó, V.; Cortés, A.; Cunha, R.A.; Lluis, C.; Franco, R. Heterodimeric adenosine receptors: a device to regulate neurotransmitter release. Cell. Mol. Life Sci., 2006, 63(21), 24272431.

[828] Schiffmann, S.N.; Vanderhaeghen, J.J. Adenosine A2 receptors regulate the gene expression of striatopallidal and striatonigral neurons. J. Neurosci., 1993, 13(3), 1080-1087.

[829] Zhang, H.; Sulzer, D. Glutamate spillover in the striatum depresses dopaminergic transmission by activating group I metabotropic glutamate receptors. J. Neurosci., 2003, 23(33), 10585-10592.

[830] Jung, K.M.; Mangieri, R.; Stapleton, C.; Kim, J.; Fegley, D.; Wallace, M.; Mackie, K.; Piomelli, D. Stimulation of endocannabinoid formation in brain slice cultures through activation of group I metabotropic glutamate receptors. Mol. Pharmacol., 2005, 68(5), 1196-1202.

[831] Shen, W.; Flajolet, M.; Greengard, P.; Surmeier, D.J. Dichotomous dopaminergic control of striatal synaptic plasticity. Science, 2008, 321(5890), 848-851.

[832] Lerner, T.N.; Horne, E.A.; Stella, N.; Kreitzer, A.C. Endocannabinoid signaling mediates psychomotor activation by adenosine $\mathrm{A}_{2 \mathrm{~A}} \mathrm{R}$ antagonists. J. Neurosci., 2010, 30(6), 2160-2164.

[833] Yin, H.H.; Lovinger, D.M. Frequency-specific and $\mathrm{D}_{2}$ receptormediated inhibition of glutamate release by retrograde endocannabinoid signaling. Proc. Natl. Acad. Sci. USA, 2006, 103(21), 8251-8256.

[834] Ferré, S.; Sebastião, A.M. Dissecting striatal adenosine-cannabinoid receptor interactions. New clues from rats over-expressing adenosine $\mathrm{A}_{2 \mathrm{~A}}$ receptors. J. Neurochem., 2016, 136(5), 897-899.

[835] Tebano, M.T.; Martire, A.; Chiodi, V.; Pepponi, R.; Ferrante, A.; Domenici, M.R.; Frank, C.; Chen, J.F.; Ledent, C.; Popoli, P. Adenosine $\mathrm{A}_{2 \mathrm{~A}}$ receptors enable the synaptic effects of cannabinoid
CB1 receptors in the rodent striatum. J. Neurochem., 2009, 110(6), 1921-1930.

[836] Chiodi, V.; Ferrante, A.; Ferraro, L.; Potenza, R.L.; Armida, M.; Beggiato, S.; Pèzzola, A.; Bader, M.; Fuxe, K.; Popoli, P.; Domenici, M.R. Striatal adenosine-cannabinoid receptor interactions in rats over-expressing adenosine $\mathrm{A}_{2 \mathrm{~A}}$ receptors. J. Neurochem., 2016, 136(5), 907-917.

[837] Brotchie, J.M. CB1 cannabinoid receptor signalling in Parkinson's disease. Curr. Opin. Pharmacol., 2003, 3(1), 54-61.

[838] Kreitzer, A.C.; Malenka, R.C. Endocannabinoid-mediated rescue of striatal LTD and motor deficits in Parkinson's disease models. Nature, 2007, 445, 643-647.

[839] Müller-Vahl, K.R.; Kolbe, H.; Schneider, U.; Emrich, H.M. Cannabis in movement disorders. Forsch. Komplementarmed., 1999, S3, 23-27.

[840] Gerdeman, G.L.; Fernández-Ruiz, J. In: Cannabinoids and the Brain; Köfalvi, A., Ed. Springer US, 2008, pp. 423-484.

[841] Venderová, K.; Růzicka, E.; Vorísek, V.; Visnovský, P. Survey on cannabis use in Parkinson's disease: subjective improvement of motor symptoms. Mov. Disord., 2004, 19(9), 1102-1106.

[842] Finseth, T.A.; Hedeman, .JL.; Brown, R.P. 2nd; Johnson, K.I.; Binder, M.S.; Kluger, B.M. Self-reported efficacy of cannabis and other complementary medicine modalities by Parkinson's disease patients in Colorado. Evid. Based Complement. Alternat. Med., 2015; 2015, 874849.

[843] Fox, S.H.; Henry, B.; Hill, M.; Crossman, A.; Brotchie, J. Stimulation of cannabinoid receptors reduces levodopa-induced dyskinesia in the MPTP-lesioned nonhuman primate model of Parkinson's disease. Mov. Disord., 2002, 17, 1180-1187.

[844] Sieradzan, K.A.; Fox, S.H.; Hill, M.; Dick, J.P.; Crossman, A.R.; Brotchie, J.M. Cannabinoids reduce levodopa-induced dyskinesia in Parkinson's disease: a pilot study. Neurology, 2001, 57, 2108-2111.

[845] Arjmand, S.; Vaziri, Z.; Behzadi, M.; Abbassian, H.; Stephens, G.J.; Shabani, M. Cannabinoids and tremor induced by motor-related Disorders: Friend or foe? Neurotherapeutics, 2015, 12(4), 778-787.

[846] Carroll, C.B.; Bain, P.G.; Teare, L.; Liu, X.; Joint, C.; Wroath, C.; Parkin, S.G.; Fox, P.; Wright, D.; Hobart, J.; Zajicek, J.P. Cannabis for dyskinesia in Parkinson disease: a randomized double-blind crossover study. Neurology, 2004, 63, 1245-1250.

[847] Gomes, C.V.; Kaster, M.P.; Tomé, A.R.; Agostinho, P.M.; Cunha, R.A. Adenosine receptors and brain diseases: neuroprotection and neurodegeneration. Biochim. Biophys. Acta, 2011, 1808(5), 13801399.

[848] de Mello Schier, A.R.; de Oliveira Ribeiro, N.P.; Coutinho, D.S.; Machado, S.; Arias-Carrión, O.; Crippa, J.A.; Zuardi, A.W.; Nardi, A.E.; Silva, A.C. Antidepressant-like and anxiolytic-like effects of cannabidiol: a chemical compound of Cannabis sativa. CNS Neuro.l Disord. Drug Targets, 2014, 13(6), 953-960.

[849] Hirvonen, J.; Goodwin, R.S.; Li, C.T.; Terry, G.E.; Zoghbi, S.S.; Morse, C.; Pike, V.W.; Volkow, N.D.; Huestis, M.A.; Innis, R.B. Reversible and regionally selective downregulation of brain cannabinoid $\mathrm{CB}_{1}$ receptors in chronic daily cannabis smokers. Mol. Psychiatry, 2012, 17(6), 642-649.

[850] Fernández-Ruiz, J.; Moreno-Martet, M.; Rodríguez-Cueto, C.; Palomo-Garo, C.; Gómez-Cañas, M.; Valdeolivas, S.; Guaza, C.; Romero, J.; Guzmán, M.; Mechoulam, R.; Ramos, J.A. Prospects for cannabinoid therapies in basal ganglia disorders. Br. J. Pharmacol., 2011, 163(7), 1365-1378.

[851] Mesnage, V.; Houeto, J.L.; Bonnet, A.M.; Clavier, I.; Arnulf, I.; Cattelin, F.; Le Fur, G.; Damier, P.; Welter, M.L.; Agid, Y. Neurokinin B, neurotensin, and cannabinoid receptor antagonists and Parkinson disease. Clin. Neuropharmacol., 2004, 27, 108-110.

[852] Pinna, A.; Bonaventura, J.; Farré, D.; Sánchez, M.; Simola, N.; Mallol, J.; Lluís, C.; Costa, G.; Baqi, Y.; Müller, C.E.; Cortés, A.; McCormick, P.; Canela, E.I.; Martínez-Pinilla, E.; Lanciego, J.L.; Casadó, V.; Armentero, M.T.; Franco, R. L-DOPA disrupts adenosine $\mathrm{A}_{2 \mathrm{~A}}$-cannabinoid $\mathrm{CB}_{1}$-dopamine $\mathrm{D}_{2}$ receptor heteromer cross-talk in the striatum of hemiparkinsonian rats: biochemical and behavioral studies. Exp. Neurol., 2014, 253, 180-191.

[853] Justinová, Z.; Solinas, M.; Tanda, G.; Redhi, G.H.; Goldberg, S.R. The endogenous cannabinoid anandamide and its synthetic analog 
$\mathrm{R}(+)$-methanandamide are intravenously self-administered by squirrel monkeys. J. Neurosci., 2005, 25(23), 5645-5650.

[854] Justinová, Z.; Ferré, S.; Redhi, G.H.; Mascia, P.; Stroik, J.; Quarta, D.; Yasar, S.; Müller, C.E.; Franco, R.; Goldberg, S.R. Reinforcing and neurochemical effects of cannabinoid $\mathrm{CB}_{1}$ receptor agonists, but not cocaine, are altered by an adenosine $\mathrm{A}_{2 \mathrm{~A}}$ receptor antagonist. Addict. Biol., 2011, 16, 405-415.

[855] Justinová, Z.; Redhi, G.H.; Goldberg, S.R.; Ferré, S. Differential effects of presynaptic versus postsynaptic adenosine $A_{2 A}$ receptor blockade on $\Delta^{9}$-tetrahydrocannabinol (THC) self-administration in squirrel monkeys. J. Neurosci., 2014, 34, 6480-6484.

[856] Orru, M.; Bakesová, J.; Brugarolas, M.; Quiroz, C.; Beaumont, V.; Goldberg, S.R.; Lluís, C.; Cortés, A.; Franco, R.; Casadó, V.; Canela, E.I.; Ferré, S. Striatal pre- and postsynaptic profile of adenosine $\mathrm{A}_{2 \mathrm{~A}}$ receptor antagonists. PLOS ONE, 2011, 6, e16088.

[857] Puntambekar, P.; van Buren, J.; Raisinghani, M.; Premkumar, L.S.; Ramkumar, V. Direct interaction of adenosine with the TRPV 1 channel protein. J. Neurosci., 2004, 24, 3663-3671.

[858] Koob, G.F.; Le Moal, M. Drug addiction, dysregulation of reward, and allostasis. Neuropsychopharmacol., 2001, 24, 97-129.

[859] Welch, S.P. Blockade of cannabinoid-induced antinociception by norbinaltorphimine, but no by N,N-diallyl-tyrosine-aibphenylalanineleucine, ICI 174,864 or naloxone in mice. J. Pharmacol. Exp. Ther., 1993, 265, 633-640.

[860] Ambrosio, E.; Martin, S.; Garcio-Llecumberri, C.; Crespo, J.A. The neurobiology of cannabinoid dependence: sex difference and potential interactions between cannabinoid and opioid system. Life Sci., 1999, 65, 687-694.

[861] Navarro, M.; Carrera; M.R.; Fratta, A.; Valverde, W.; Cossu, O.; Fattore, G.; Chowen, L.; Gómez, J.A.; del Arco, R.; Villanúa, I.; Maldonado, M.A.; Koob, R.G.F.; Rodríguez de Fonseca, F. Functional interaction between opioid and cannabinoid receptors in drug self-administration. J. Neurosci., 2001, 21, 5344-5350.

[862] Capasso, A.; Gallo, C. Molecules Acting on $\mathrm{CB}_{1}$ Receptor and their Effects on Morphine Withdrawal In Vitro. Open Biochem. J., 2009, 3, 78-84.

[863] Manzanares, J.; Corchero, J.; Romero, J.; Fernandez-Ruiz, J.J.; Ramos, J.A.; Fuentes J.A. Pharmacological and biochemical interactions between opioids and cannabinoids. Trends Pharmacol. Sci., 1999, 20, 287-294.

[864] Massi, P.; Vaccani, A.; Romorini, S.; Parolaro, D. Comparative characterization in the rat of the interaction between cannabinoids and opiates for their immunosuppressive and analgesic effects. $J$. Neuroimmunol., 2001, 117, 116-124.

[865] Cichewicz, D.L. Synergistic interactions between cannabinoid and opioid analgesics. Life Sci., 2004, 74(11), 1317-1324.

[866] Rios, C.; Gomes, I.; Devi, L.A. mu opioid and $\mathrm{CB}_{1}$ cannabinoid receptor interactions: reciprocal inhibition of receptor signaling and neuritogenesis. Br. J. Pharmacol., 2006, 148, 387-395.

[867] Rios, C.; Gomes, I.; Devi, L.A. mu opioid and $\mathrm{CB}_{1}$ cannabinoid receptor interactions: reciprocal inhibition of receptor signaling and neuritogenesis. Br. J. Pharmacol., 2006, 148, 387-395.

[868] Bushlin, I.; Gupta, A.; Stockton, S.D. Jr; Miller, L.K.; Devi, L.A. Dimerization with cannabinoid receptors allosterically modulates delta opioid receptor activity during neuropathic pain. PLoS ONE, 2012, 7(12), e49789.

[869] Fichna, J.; Schicho, R.; Andrews, C.N.; Bashashati, M.; Klompus, M.; McKay, D.M.; Sharkey, K.A.; Zjawiony, J.K.; Janecka, A.; Storr, M.A. Salvinorin A inhibits colonic transit and neurogenic ion transport in mice by activating kappa-opioid and cannabinoid receptors. Neurogastroenterol. Motil., 2009, 21, 1326-e128.

[870] Seely, K.A.; Brents, L.K.; Franks, L.N.; Rajasekaran, M.; Zimmerman, S.M.; Fantegrossi, W.E.; Prather, P.L. AM-251 and rimonabant act as direct antagonists at $\mu$-opioid receptors: implications for opioid/cannabinoid interaction studies. Neuropharmacology, 2012, 63(5), 905-915.

[871] Zádor, F.; Ötvös, F.; Benyhe, S.; Zimmer, A.; Páldy, E. Inhibition of forebrain $\mu$-opioid receptor signaling by low concentrations of rimonabant does not require cannabinoid receptors and directly involves $\mu$-opioid receptors. Neurochem. Int., 2012, 61(3), 378-388.
[872] Zádor, F.; Kocsis, D.; Borsodi, A.; Benyhe, S. Micromolar concentrations of rimonabant directly inhibits delta opioid receptor specific ligand binding and agonist-induced G-protein activity. Neurochem. Int., 2014, 67, 14-22.

[873] Zádor, F.; Lénárt, N.; Csibrány, B.; Sántha, M.; Molnár, M.; Tuka, B.; Samavati, R.; Klivényi, P.; Vécsei, L.; Marton, A.; Vizler, C.; Nagy, G.M.; Borsodi, A.; Benyhe, S.; Páldy, E. Low dosage of rimonabant leads to anxiolytic-like behavior via inhibiting expression levels and G-protein activity of kappa opioid receptors in a cannabinoid receptor independent manner. Neuropharmacology, 2015, 89, 298-307.

[874] Hart, S.; Fischer, O.M.; Ullrich, A. Cannabinoids induce cancer cell proliferation via tumor necrosis factor alpha-converting enzyme (TACE/ADAM17)-mediated transactivation of the epidermal growth factor receptor. Cancer Res., 2004, 64(6), 1943-1950.

[875] Williams, E.J.; Walsh, F.S.; Doherty, P. The FGF receptor uses the endocannabinoid signaling system to couple to an axonal growth response. J. Cell. Biol., 2003, 160(4), 481-486.

[876] Berghuis, P.; Rajnicek, A.M.; Morozov, Y.M.; Ross, R.A.; Mulder, J.; Urbán, G.M.; Monory, K.; Marsicano, G.; Matteoli, M.; Canty, A.; Irving, A.J.; Katona, I.; Yanagawa, Y.; Rakic, P.; Lutz, B.; Mackie, K.; Harkany, T. Hardwiring the brain: endocannabinoids shape neuronal connectivity. Science, 2007, 316(5828), 1212-1216.

[877] Dalton, G.D.; Howlett, A.C. Cannabinoid CB $_{1}$ receptors transactivate multiple receptor tyrosine kinases and regulate serine/threonine kinases to activate ERK in neuronal cells. Br. J. Pharmacol., 2012, 165(8), 2497-2511.

[878] Kim, W.; Lao, Q.; Shin, Y.K.; Carlson, O.D.; Lee, E.K.; Gorospe, M.; Kulkarni, R.N.; Egan, J.M. Cannabinoids induce pancreatic $\beta$-cell death by directly inhibiting insulin receptor activation. Sci. Signal, 2012, 5(216), ra23.

[879] Berghuis, P.; Dobszay, M.B.; Wang, X.; Spano, S.; Ledda, F.; Sousa, K.M.; Schulte, G.; Ernfors, P.; Mackie, K.; Paratcha, G.; Hurd, Y.L.; Harkany, T. Endocannabinoids regulate interneuron migration and morphogenesis by transactivating the TrkB receptor. Proc. Natl. Acad. Sci. USA, 2005, 102(52), 19115-19120.

[880] Pinheiro, B.S.; Lemos, C.; Neutzling-Kaufmann, F.; Marques, J.M.; da Silva-Santos, C.S.; Carvalho, E.; Mackie, K.; Rodrigues, R.J.; Cunha, R.A.; Köfalvi, A. Hierarchical glucocorticoidendocannabinoid interplay regulates the activation of the nucleus accumbens by insulin. Brain Res. Bull., 2016, 124, 222-230.

[881] Kim, D.; Thayer, S.A. Cannabinoids inhibit the formation of new synapses between hippocampal neurons in culture. J. Neurosci., 2001, 21(10), RC146.

[882] Díaz-Alonso, J.; Guzmán, M.; Galve-Roperh, I. Endocannabinoids via $\mathrm{CB}_{1}$ receptors act as neurogenic niche cues during cortical development. Philos. T. R. Soc. B., 2012, 367(1607), 3229-3241.

[883] Galve-Roperh, I.; Palazuelos, J.; Aguado, T.; Guzmán, M. The endocannabinoid system and the regulation of neural development: potential implications in psychiatric disorders. Eur. Arch. Psychiatry Clin. Neurosci., 2009, 259(7), 371-382.

[884] Campolongo, P.; Trezza, V.; Palmery, M.; Trabace, L.; Cuomo, V. Developmental exposure to cannabinoids causes subtle and enduring neurofunctional alterations. Int. Rev. Neurobiol., 2009, 85, 117-133.

[885] Psychoyos, D.; Vinod, K.Y. Marijuana, Spice 'herbal high', and early neural development: implications for rescheduling and legalization. Drug Test Anal., 2013, 5(1), 27-45.

[886] Calvigioni, D.; Hurd, Y.L.; Harkany, T.; Keimpema, E. Neuronal substrates and functional consequences of prenatal cannabis exposure. Eur. Child Adolesc. Psychiatry, 2014, 23(10), 931-941.

[887] Tortoriello, G.; Morris, C.V.; Alpár, A.; Fuzik, J.; Shirran, S.L.; Calvigioni, D.; Keimpema, E.; Botting, C.H.; Reinecke, K.; Herdegen, T.; Courtney, M.; Hurd, Y.L.; Harkany, T. Miswiring the brain: $\Delta^{9}$-tetrahydrocannabinol disrupts cortical development by inducing an SCG10/stathmin-2 degradation pathway. EMBO J., 2014, 33(7), 668-685.

[888] Alpár, A.; Di Marzo, V.; Harkany, T. At the tip of an iceberg: prenatal marijuana and its possible relation to neuropsychiatric outcome in the offspring. Biol. Psychiatry, 2016, 79(7), e33-45.

[889] Volkow, N.D.; Swanson, J.M.; Evins, A.E.; DeLisi, L.E.; Meier, M.H.; Gonzalez, R.; Bloomfield, M.A.; Curran, H.V.; Baler, R. 
Effects of cannabis use on human behavior, including cognition, motivation, and psychosis: A review. JAMA Psychiatry, 2016, 73(3), 292-297.

[890] Bernard, C.; Milh, M.; Morozov, Y.M.; Ben-Ari, Y.; Freund, T.F.; Gozlan, H. Altering cannabinoid signaling during development disrupts neuronal activity. Proc. Natl. Acad. Sci. USA, 2005, 102(26), 9388-9393.

[891] Zurolo, E.; Iyer, A.M.; Spliet, W.G.; van Rijen, P.C.; Troost, D.; Gorter, J.A.; Aronica, E. $\mathrm{CB}_{1}$ and $\mathrm{CB}_{2}$ cannabinoid receptor expression during development and in epileptogenic developmental pathologies. Neuroscience, 2010, 170(1), 28-41.

[892] Malenczyk, K.; Jazurek, M.; Keimpema, E.; Silvestri, C.; Janikiewicz, J.; Mackie, K.; Di Marzo, V.; Redowicz, M.J.; Harkany, T.; Dobrzyn, A. $\mathrm{CB}_{1}$ cannabinoid receptors couple to focal adhesion kinase to control insulin release. J. Biol. Chem., 2013, 288(45), 32685-32699.

[893] Labouèbe, G.; Liu, S.; Dias, C.; Zou, H.; Wong, J.C.; Karunakaran, S.; Clee, S.M.; Phillips, A.G.; Boutrel, B.; Borgland, S.L. Insulin induces long-term depression of ventral tegmental area dopamine neurons via endocannabinoids. Nat. Neurosci., 2013, 16(3), 300-308.

[894] Coke, C.J.; Scarlett, K.A.; Chetram, M.A.; Jones, K.J.; Sandifer, B.J.; Davis, A.S.; Marcus, A.I.; Hinton, C.V. Simultaneous activation of induced heterodimerization between CXCR4 chemokine receptor and cannabinoid receptor 2 (CB2) reveals a mechanism for regulation of tumor progression. J. Biol. Chem., 2016, 291(19), 9991-10005.

[895] Fagan, S.G.; Campbell, V.A. The influence of cannabinoids on generic traits of neurodegeneration. Br. J. Pharmacol., 2014, 171(6), $1347-1360$.

[896] Gowran, A.; Noonan, J.; Campbell, V.A. The multiplicity of action of cannabinoids: implications for treating neurodegeneration. CNS Neurosci. Ther., 2011, 17(6), 637-644.

[897] Fowler, C.J.; Rojo, M.L.; Rodriguez-Gaztelumendi, A. Modulation of the endocannabinoid system: neuroprotection or neurotoxicity? Exp. Neurol., 2010, 224(1), 37-47.

[898] Nagayama, T.; Sinor, A.D.; Simon, R.P.; Chen, J.; Graham, S.H.; Jin, K.; Greenberg, D.A. Cannabinoids and neuroprotection in global and focal cerebral ischemia and in neuronal cultures. J. Neurosci., 1999, 19, 2987-2995.

[899] Bai, F.; Guo, F.; Jiang, T.; Wei, H.; Zhou, H.; Yin, H.; Zhong, H.; Xiong, L.; Wang, Q. Arachidonyl-2-chloroethylamide alleviates cerebral ischemia injury through glycogen synthase kinase-3 $\beta$ mediated mitochondrial biogenesis and functional improvement. Mol. Neurobiol., 2016, in press, doi:10.1007/s12035-016-9731-7

[900] van der Stelt, M.; Veldhuis, W.B.; Bär, P.R.; Veldink, G.A.; Vliegenthart, J.F.; Nicolay, K. Neuroprotection by Delta9tetrahydrocannabinol, the main active compound in marijuana, against ouabain-induced in vivo excitotoxicity. J. Neurosci., 2001, 21(17), 6475-6479.

[901] Ramírez, B.G.; Blázquez, C.; Gómez del Pulgar, T.; Guzmán, M.; de Ceballos, M.L. Prevention of Alzheimer's disease pathology by cannabinoids: neuroprotection mediated by blockade of microglial activation. J. Neurosci. Off. J. Soc. Neurosci. 2005, 25, 1904-1913.

[902] Marsicano, G.; Moosmann, B.; Hermann, H.; Lutz, B.; Behl, C. Neuroprotective properties of cannabinoids against oxidative stress: role of the cannabinoid receptor $\mathrm{CB}_{1}$. J. Neurochem., 2002, 80(3), 448-456.

[903] Fernández-Ruiz, J.; García, C.; Sagredo, O.; Gómez-Ruiz, M.; de Lago, E. The endocannabinoid system as a target for the treatment of neuronal damage. Expert Opin. Ther. Targets, 2010, 14(4), 387-404.

[904] Martín-Moreno, A.M.; Brera, B.; Spuch, C.; Carro, E.; García-García, L.; Delgado, M.; Pozo, M.A.; Innamorato, N.G.; Cuadrado, A.; de Ceballos, M.L. Prolonged oral cannabinoid administration prevents neuroinflammation, lowers $\beta$-amyloid levels and improves cognitive performance in Tg APP 2576 mice. J. Neuroinflammation, 2012, 9, 8.

[905] Gómez-Gálvez, Y.; Palomo-Garo, C.; Fernández-Ruiz, J.; García, C. Potential of the cannabinoid $\mathrm{CB}_{2}$ receptor as a pharmacological target against inflammation in Parkinson's disease. Prog. Neuropsychopharmacol. Biol. Psychiatry, 2016, 64, 200-208.

[906] Rocchetti, M.; Crescini, A.; Borgwardt, S.; Caverzasi, E.; Politi, P.; Atakan, Z.; Fusar-Poli, P. Is cannabis neurotoxic for the healthy brain? A meta-analytical review of structural brain alterations in nonpsychotic users. Psychiatry Clin. Neurosci., 2013, 67(7), 483-492.

[907] Landfield, P.W.; Cadwallader, L.B.; Vinsant, S. Quantitative changes in hippocampal structure following long-term exposure to THC: possible mediation by glucocorticoid systems. Brain Res., 1988, 443, 47-62.

[908] Demirakca, T.; Sartorius, A.; Ende, G.; Meyer, N.; Welzel, H.; Skopp, G.; Mann, K.; Hermann, D. Diminished gray matter in the hippocampus of cannabis users: possible protective effects of cannabidiol. Drug Alcohol Depend., 2011, 114(2-3), 242-245.

[909] Cousijn, J.; Wiers, R.W.; Ridderinkhof, K.R.; van den Brink, W.; Veltman, D.J.; Goudriaan, A.E. Grey matter alterations associated with cannabis use: results of a VBM study in heavy cannabis users and healthy controls. Neuroimage, 2012, 59(4), 3845-3851.

[910] Battistella, G.; Fornari, E.; Annoni, J.M.; Chtioui, H.; Dao, K.; Fabritius, M.; Favrat, B.; Mall, J.F.; Maeder, P.; Giroud, C. Longterm effects of cannabis on brain structure. Neuropsychopharmacol., 2014, 39(9), 2041-2048.

[911] Downer, E.J.; Fogarty, M.P.; Campbell, V.A. Tetrahydrocannabinolinduced neurotoxicity depends on $\mathrm{CB}_{1}$ receptor-mediated c-Jun Nterminal kinase activation in cultured cortical neurons. Br. J. Pharmacol., 2003, 140(3), 547-557.

[912] Downer, E.J.; Gowran, A.; Murphy, A.C.; Campbell, V.A. The tumour suppressor protein, p53, is involved in the activation of the apoptotic cascade by Delta9-tetrahydrocannabinol in cultured cortical neurons. Eur. J. Pharmacol., 2007, 564(1-3), 57-65.

[913] Chan, G.C.; Hinds, T.R.; Impey, S.; Storm, D.R. Hippocampal neurotoxicity of Delta9-tetrahydrocannabinol. J. Neurosci., 1998, 18, 5322-5332.

[914] Chen, R.; Zhang, J.; Fan, N.; Teng, Z.Q.; Wu, Y.; Yang, H.; Tang Y.P.; Sun, H.; Song, Y.; Chen, C. $\Delta^{9}$-THC-caused synaptic and memory impairments are mediated through COX-2 signaling. Cell, 2013, 155(5), 1154-1165.

[915] Zhang, J.; Chen, C. Endocannabinoid 2-arachidonoylglycerol protects neurons by limiting COX-2 elevation. J. Biol. Chem., 2008, 283, 22601-22611.

[916] Tselnicker, I.; Keren, O.; Hefetz, A.; Pick, C.G.; Sarne, Y. A single low dose of tetrahydrocannabinol induces long-term cognitive deficits. Neurosci. Lett., 2007, 411(2), 108-111.

[917] Machado, V.M.; Morte, M.I.; Carreira, B.P.; Azevedo, M.M.; Takano, J.; Iwata, N.; Saido, T.C.; Asmussen, H.; Horwitz, A.R.; Carvalho, C.M.; Araújo, I.M. Involvement of calpains in adult neurogenesis: implications for stroke. Front. Cell. Neurosci., 2015, 9 , 22.

[918] Prenderville, J.A.; Kelly, Á.M.; Downer, E.J. The role of cannabinoids in adult neurogenesis. Br. J. Pharmacol., 2015, 172(16), 3950-3963.

[919] Yau, S.Y.; Li, A.; So, K.F. Involvement of adult hippocampal neurogenesis in learning and forgetting. Neural. Plast., 2015, 2015, 717958.

[920] Aguado, T.; Monory, K.; Palazuelos, J.; Stella, N.; Cravatt, B.; Lutz, B.; Marsicano, G.; Kokaia, Z.; Guzman, M.; Galve-Roperh, I. The endocannabinoid system drives neural progenitor proliferation. FASEB J., 2005, 19, 1704-1706.

[921] Aguado, T.; Palazuelos, J.; Monory, K.; Stella, N.; Cravatt, B.; Lutz, B.; Marsicano, G.; Kokaia, Z.; Guzman, M.; Galve-Roperh, I. The endocannabinoid system promotes astroglial differentiation by acting on neural progenitor cells. J. Neurosci., 2006, 26, 1551-1561.

[922] Jiang, W.; Zhang, Y.; Xiao, L.; Van, C.J.; Ji, S.P.; Bai, G.; Zhang, X. Cannabinoids promote embryonic and adult hippocampus neurogenesis and produce anxiolytic- and antidepressant-like effects. J. Clin. Invest., 2005, 115, 3104-3116.

[923] Galve-Roperh, I.; Aguado, T.; Palazuelos, J.; Guzman, M. The endocannabinoid system and neurogenesis in health and disease. Neuroscientist, 2007, 13, 109-114.

[924] Xapelli, S.; Agasse, F.; Sardà-Arroyo, L.; Bernardino, L.; Santos, T.; Ribeiro, F.F.; Valero, J.; Bragança, J.; Schitine, C.; de Melo Reis, R.A.; Sebastião, A.M.; Malva, J.O. Activation of type 1 cannabinoid receptor $\left(\mathrm{CB}_{1} \mathrm{R}\right)$ promotes neurogenesis in murine subventricular zone cell cultures. PLoS ONE, 2013, 8(5), e63529. 
[925] Rueda, D.; Navarro, B.; Martinez-Serrano, A.; Guzman, M.; GalveRoperh, I. The endocannabinoid anandamide inhibits neuronal progenitor cell differentiation through attenuation of the Rap1/BRaf/ERK pathway. J. Biol. Chem., 2002, 277, 46645-46650.

[926] Mackowiak, M.; Chocyk, A.; Markowicz-Kula, K.; Wedzony, K. Acute activation of $\mathrm{CB}_{1}$ cannabinoid receptors transiently decreases PSA-NCAM expression in the dentate gyrus of the rat hippocampus. Brain Res., 2007, 1148, 43-52.

[927] Goncalves, M.B.; Suetterlin, P.; Yip, P.; Molina-Holgado, F.; Walker, D.J.; Oudin, M.J.; Zentar, M.P.; Pollard, S.; Yáñez-Muñoz, R.J.; Williams, G.; Walsh, F.S.; Pangalos, M.N.; Doherty, P. A diacylglycerol lipase- $\mathrm{CB}_{2}$ cannabinoid pathway regulates adult subventricular zone neurogenesis in an age-dependent manner. Mol. Cell. Neurosci., 2008, 38(4), 526-536.

[928] Avraham, H.K.; Jiang, S.; Fu, Y.; Rockenstein, E.; Makriyannis, A.; Zvonok, A.; Masliah, E.; Avraham, S. The cannabinoid $\mathrm{CB}_{2}$ receptor agonist AM1241 enhances neurogenesis in GFAP/Gp120 transgenic mice displaying deficits in neurogenesis. Br. J. Pharmacol., 2014, 171(2), 468-479.

[929] Parolaro, D.; Realini, N.; Vigano, D.; Guidali, C.; Rubino, T. The endocannabinoid system and psychiatric disorders. Exp. Neurol., 2010, 224(1), 3-14.

[930] Arseneault, L.; Cannon, M.; Witton, J.; Murray, R.M. Causal association between cannabis and psychosis: examination of the evidence. Br. J. Psychiatry, 2004, 184, 110-117.

[931] Murray, R.M.; Morrison, P.D.; Henquet, C.; Di Forti, M. Cannabis, the mind and society: the hash realities. Nat. Rev. Neurosci., 2007, 8, 885-895.

[932] Hill, M.N. Clearing the smoke: what do we know about adolescent cannabis use and schizophrenia? J Psychiatry Neurosci., 2014, 39(2), 75-77.

[933] Saha, S.; Scott, J.G.; Varghese, D.; Degenhardt, L.; Slade, T.; McGrath, J.J. The association between delusional-like experiences, and tobacco, alcohol or cannabis use: a nationwide population-based survey. BMC Psychiatry, 2011, 11, 202.

[934] Compton, M.T.; Furman, A.C.; Kaslow, N.J. Lower negative symptom scores among cannabis-dependent patients with schizophrenia-spectrum disorders: preliminary evidence from an African American first-episode sample. Schizophr. Res., 2004, 71, 61-64.

[935] Rehman, I.U.; Farooq, S. Cannabis abuse in patients with schizophrenia: pattern and effects on symptomatology. J. Coll. Physicians Surg. Pak., 2007, 17(3), 158-161.

[936] Henquet, C.; van Os, J.; Kuepper, R.; Delespaul, P.; Smits, M.; Campo, J.A.; Myin-Germeys, I. Psychosis reactivity to cannabis use in daily life: an experience sampling study. Br. J. Psychiatry, 2010, 196(6), 447-453.

[937] Linszen, D.H.; Dingemans, P.M.; Lenior, M.E. Cannabis abuse and the course of recent-onset schizophrenic disorders. Arch. Gen. Psychiatry, 1994, 51, 273-279.

[938] Caspari, D. Cannabis and schizophrenia: results of a follow-up study. Eur. Arch. Psychiatry Clin. Neurosci., 1999, 249, 45-49.

[939] Grech, A.; van Os, J.; Jones, P.B.; Lewis, S.W.; Murray, R.M. Cannabis use and outcome of recent onset psychosis. Eur. Psychiatry, 2005, 20, 349-353.

[940] Zammit, S.; Allebeck, P.; Andreasson, S.; Lundberg, I.; Lewis, G. Self reported cannabis use as a risk factor for schizophrenia in Swedish conscripts of 1969: historical cohort study. BMJ, 2002, 325(7374), 1199.

[941] Hill, M.N. Perspective: Be clear about the real risks. Nature, 2015, 525(7570), S14.

[942] Power, R.A.; Verweij, K.J.; Zuhair, M.; Montgomery, G.W.; Henders, A.K.; Heath, A.C.; Madden, P.A.; Medland, S.E.; Wray, N.R.; Martin, N.G. Genetic predisposition to schizophrenia associated with increased use of cannabis. Mol. Psychiatry, 2014, 19(11), 12011204.

[943] American Psychiatric Association. Diagnostic and Statistical Manual, $5^{\text {th }}$ ed.; American Psychiatric Association: Arlington, 2013.

[944] Boydell, J.; van Os, J.; Lambri, M.; Castle, D.; Allardyce, J.; McCreadie, R.G.; Murray, R.M. Incidence of schizophrenia in south- east London between 1965 and 1997. Br. J. Psychiatry, 2003, 182, 45-49.

[945] Di Forti, M.; Marconi, A.; Carra, E.; Fraietta, S.; Trotta, A.; Bonomo, M.; Bianconi, F.; Gardner-Sood, P.; O'Connor, J.; Russo, M.; Stilo, S.A.; Marques, T.R.; Mondelli, V.; Dazzan, P.; Pariante, C.; David, A.S.; Gaughran, F.; Atakan, Z.; Iyegbe, C.; Powell, J.; Morgan, C.; Lynskey, M.; Murray, R.M. Proportion of patients in south London with first-episode psychosis attributable to use of high potency cannabis: a case-control study. Lancet Psychiatry, 2015, 2(3), 233238.

[946] van Amsterdam, J.; Brunt, T.; van den Brink, W. The adverse health effects of synthetic cannabinoids with emphasis on psychosis-like effects. J. Psychopharmacol., 2015, 29(3), 254-263.

[947] Schneider, M.; Koch, M. Chronic pubertal, but not adult chronic cannabinoid treatment impairs sensorimotor gating, recognitionmemory, and the performance in a progressive ratio task in adult rats. Neuropsychopharmacol., 2003, 28, 1760-1769.

[948] Wiley, J.L.; Marusich, J.A.; Lefever, T.W.; Antonazzo, K.R.; Wallgren, M.T.; Cortes, R.A.; Patel, P.R.; Grabenauer, M.; Moore, K.N.; Thomas, B.F. AB-CHMINACA, AB-PINACA, and FUBIMINA: Affinity and potency of novel synthetic cannabinoids in producing $\Delta^{9}$-tetrahydrocannabinol-like effects in mice. $J$. Pharmacol. Exp. Ther., 2015, 354(3), 328-339.

[949] Bernson-Leung, M.E.; Leung, L.Y.; Kumar, S. Synthetic cannabis and acute ischemic stroke. J. Stroke Cerebrovasc. Dis., 2014, 23(5), 1239-1241.

[950] Shanks, K.G.; Behonick, G.S. Death after use of the synthetic cannabinoid 5F-AMB. Forensic Sci. Int., 2016, 262, e21-e24.

[951] Papanti, D.; Schifano, F.; Botteon, G.; Bertossi, F.; Mannix, J.; Vidoni, D.; Impagnatiello, M.; Pascolo-Fabrici, E.; Bonavigo, T. "Spiceophrenia": a systematic overview of "spice"-related psychopathological issues and a case report. Hum. Psychopharmacol., 2013, 28(4), 379-389.

[952] Sundram, S. Cannabis and neurodevelopment: implications for psychiatric disorders. Hum. Psychopharmacol., 2006, 21(4), 245-254.

[953] Chadwick, B.; Miller, M.L.; Hurd, Y.L. Cannabis use during adolescent development: Susceptibility to psychiatric illness. Front. Psychiatry, 2013, 4, 129.

[954] Renard, J.; Krebs, M.O.; Le Pen, G.; Jay, T.M. Long-term consequences of adolescent cannabinoid exposure in adult psychopathology. Front. Neurosci., 2014, 8, 361.

[955] Rubino, T.; Parolaro, D. The impact of exposure to cannabinoids in adolescence: Insights from animal models. Biol. Psychiatry, 2016, 79(7), 578-585

[956] Terranova, J.P.; Storme, J.J.; Lafon, N.; Pério, A.; Rinaldi-Carmona, M.; Le Fur, G.; Soubrié, P. Improvement of memory in rodents by the selective $\mathrm{CB}_{1}$ cannabinoid receptor antagonist, SR141716. Psychopharmacology (Berl.), 1996, 126, 165-172.

[957] Nava, F.; Carta, G.; Battesi, A.M.; Gessa, G.L. D ${ }_{2}$ dopamine receptors enable D-9-tetrahydrocannabinol induced memory impairment and reduction of hippocampal extracellular acetylcholine concentration. Br. J. Pharmacol., 2000, 130, 1201-1210.

[958] Mishima, K.; Egashira, N.; Matsumoto, Y.; Iwasaki, K.; Fujiwara, M Involvement of reduced acetylcholine release in $\Delta^{9}$ tetrahydrocannabinol-induced impairment of spatial memory in the 8arm radial maze. Life Sci., 2002, 72, 397-407.

[959] Blanco, C.; Hasin, D.S.; Wall, M.M.; Flórez-Salamanca, L.; Hoertel, N.; Wang, S.; Kerridge, B.T.; Olfson, M. Cannabis use and risk of psychiatric disorders: Prospective evidence from a US national longitudinal study. JAMA Psychiatry, 2016, 73(4), 388-395.

[960] Witkin, J.M.; Tzavara, E.T.; Davis, R.J.; Li, X.; Nomikos, G.G. A therapeutic role for cannabinoid CB1 receptor antagonists in major depressive disorders. Trends Pharmacol. Sci., 2005a, 26(12), 609617.

[961] Witkin, J.M.; Tzavara, E.T.; Nomikos, G.G. A role for cannabinoid $\mathrm{CB}_{1}$ receptors in mood and anxiety disorders. Behav. Pharmacol., 2005b, 16(5-6), 315-331.

[962] Tzavara, E.T.; Witkin, J.M. In: Cannabinoids and the Brain; Köfalvi, A., Ed. Springer US, 2008, pp. 529-558. 
[963] Degroot, A. In: Cannabinoids and the Brain; Köfalvi, A., Ed. Springer US, 2008, pp. 559-572.

[964] Riebe, C.J.; Wotjak, C.T. Endocannabinoids and stress. Stress, 2011, 14(4), 384-397.

[965] Bitencourt, R.M.; Pamplona, F.A.; Takahashi, R.N. A current overview of cannabinoids and glucocorticoids in facilitating extinction of aversive memories: potential extinction enhancers. Neuropharmacology, 2013, 64, 389-395.

[966] Hill, M.N.; McEwen, B.S. Endocannabinoids: The silent partner of glucocorticoids in the synapse. Proc. Natl. Acad. Sci. USA, 2009, 106, 4579-4580.

[967] Hill, M.N.; McLaughlin, R.J.; Pan, B.; Fitzgerald, M.L.; Roberts, C.J.; Lee, T.T.-Y.; Karatsoreos, I.N.; Mackie, K.; Viau, V.; Pickel, V.M.; McEwen, B.S.; Liu, Q.; Gorzalka, B.B.; Hillard, C.J. Recruitment of prefrontal cortical endocannabinoid signaling by glucocorticoids contributes to termination of the stress response. $J$. Neurosci. Off. J. Soc. Neurosci., 2011, 31, 10506-10515.

[968] Hill, M.N.; Tasker, J.G. Endocannabinoid signaling, glucocorticoidmediated negative feedback, and regulation of the hypothalamicpituitary-adrenal axis. Neuroscience, 2012, 204, 5-16.

[969] Marsicano, G.; Wotjak, C.T.; Azad, S.C.; Bisogno, T.; Rammes, G.; Cascio, M.G.; Hermann, H.; Tang, J.; Hofmann, C.; Zieglgänsberger, W.; Di Marzo, V.; Lutz, B. The endogenous cannabinoid system controls extinction of aversive memories. Nature, 2002, 418, 530534.

[970] Atsak, P.; Hauer, D.; Campolongo, P.; Schelling, G.; McGaugh, J.L.; Roozendaal, B. Glucocorticoids interact with the hippocampal endocannabinoid system in impairing retrieval of contextual fear memory. Proc. Natl. Acad. Sci. USA, 2012, 109(9), 3504-3509.

[971] Bitencourt, R.M.; Pamplona, F.A.; Takahashi, R.N. Corticosteroidendocannabinoid loop supports decrease of fear-conditioned response in rats. Eur. Neuropsychopharmacol., 2014, 24(7), 1091-1102.

[972] Belkin, M.R.; Schwartz, T.L. Alpha-2 receptor agonists for the treatment of posttraumatic stress disorder. Drugs in Context, 2015, 4, 212286.

[973] Soria-Gómez, E.; Busquets-Garcia, A.; Hu, F.; Mehidi, A.; Cannich, A.; Roux, L.; Louit, I.; Alonso, L.; Wiesner, T.; Georges, F.; Verrier, D.; Vincent, P.; Ferreira, G.; Luo, M.; Marsicano, G. Habenular $\mathrm{CB}_{1}$ Receptors Control the Expression of Aversive Memories. Neuron, 2015, 88(2), 306-313.

[974] Belendiuk, K.A.; Baldini, L.L.; Bonn-Miller, M.O. Narrative review of the safety and efficacy of marijuana for the treatment of commonly state-approved medical and psychiatric disorders. Addict. Sci. Clin. Pract., 2015, 10, 10.

[975] Betthauser, K.; Pilz, J.; Vollmer, L.E. Use and effects of cannabinoids in military veterans with posttraumatic stress disorder. Am. J. Health Syst. Pharm., 2015, 72(15), 1279-1284.

[976] McEwen, B.S.; Bowles, N.P.; Gray, J.D.; Hill, M.N.; Hunter, R.G.; Karatsoreos, I.N.; Nasca, C. Mechanisms of stress in the brain. Nat. Neurosci., 2015, 18(10), 1353-1363.

[977] Bowles, N.P.; Karatsoreos, I.N.; Li, X.; Vemuri, V.K.; Wood, J.A.; Li, Z.; Tamashiro, K.L.; Schwartz, G.J.; Makriyannis, A.M.; Kunos, G.; Hillard, C.J.; McEwen, B.S.; Hill, M.N. A peripheral endocannabinoid mechanism contributes to glucocorticoid-mediated metabolic syndrome. Proc. Natl. Acad. Sci. USA, 2015, 112(1), 285290.

[978] van den Bossche, I.; Vanheel, B. Influence of cannabinoids on the delayed rectifier in freshly dissociated smooth muscle cells of the rat aorta. Br. J. Pharmacol., 2000, 131(1), 85-93.

[979] Breivogel, C.S.; Griffin, G.; Di Marzo, V.; Martin, B.R. Evidence for a new $\mathrm{G}$ protein-coupled cannabinoid receptor in mouse brain. $\mathrm{Mol}$. Pharmacol., 2001, 60(1), 155-163.

[980] Köfalvi, A.; Vizi, E.S.; Ledent, C.; Sperlágh, B. Cannabinoids inhibit the release of $\left[{ }^{3} \mathrm{H}\right]$ glutamate from rodent hippocampal synaptosomes via a novel CB1 receptor-independent action. Eur. J. Neurosci., 2003, 18, 1973-1978.

[981] Akopian, A.N.; Ruparel, N.B.; Patwardhan, A.; Hargreaves, K.M. Cannabinoids desensitize capsaicin and mustard oil responses in sensory neurons via TRPA ${ }_{1}$ activation. J. Neurosci., 2008, 28, 10641075.
[982] Ahrens, J.; Demir, R.; Leuwer, M.; de la Roche, J.; Krampfl, K.; Foadi, N.; Karst, M.; Haeseler, G. The Nonpsychotropic Cannabinoid Cannabidiol Modulates and Directly Activates Alpha-1 and Alpha-1Beta Glycine Receptor Function. Pharmacology, 2009, 83(4), 217222.

[983] Taylor, L.; Christou, I.; Kapellos, T.S.; Buchan, A.; Brodermann, M.H.; Gianella-Borradori, M.; Russell, A.; Iqbal, A.J.; Greaves, D.R. Primary macrophage chemotaxis induced by cannabinoid receptor 2 agonists occurs independently of the $\mathrm{CB}_{2}$ receptor. Sci. Rep., 2015, 5, 10682.

[984] Eubanks, L.M.; Rogers, C.J.; Beuscher, A.E. 4th; Koob, G.F.; Olson, A.J.; Dickerson, T.J.; Janda, K.D. A molecular link between the active component of marijuana and Alzheimer's disease pathology. Mol. Pharm., 2006, 3(6), 773-777.

[985] Fisar, Z. Inhibition of monoamine oxidase activity by cannabinoids. Naunyn. Schmiedebergs Arch. Pharmacol., 2010, 381(6), 563-572.

[986] Kaplan, B.L.; Rockwell, C.E.; Kaminski, N.E. Evidence for cannabinoid receptor-dependent and -independent mechanisms of action in leukocytes. J. Pharmacol. Exp. Ther., 2003, 306(3), 10771085.

[987] Burstein, S.H.; Audette, C.A.; Breuer, A.; Devane, W.A.; Colodner, S.; Doyle, S.A.; Mechoulam, R. Synthetic nonpsychotropic cannabinoids with potent antiinflammatory, analgesic and leukocyte antiadhesion activities. J. Med. Chem., 1992, 35, 3135-3141.

[988] Liu, J.; Li, H.; Burstein, S.H.; Zurier, R.B.; Chen, J.D. Activation and binding of peroxisome proliferator-activated receptor gamma by synthetic cannabinoid ajulemic acid. Mol. Pharmacol., 2003, 63(5), 983-992.

[989] Houseknecht, K.L.; Cole, B.M.; Steele, P.J. Peroxisome proliferatoractivated receptor gamma (PPARgamma) and its ligands: a review. Domest. Anim. Endocrinol., 2002, 22, 1-23.

[990] Burstein. S. PPAR-gamma: a nuclear receptor with affinity for cannabinoids. Life Sci., 2005, 77(14), 1674-1684.

[991] Rockwell, C.E.; Snider, N.T.; Thompson, J.T.; Vanden Heuvel, J.P.; Kaminski, N.E. Interleukin-2 suppression by 2-arachidonyl glycerol is mediated through peroxisome proliferator-activated receptor gamma independently of cannabinoid receptors 1 and 2. Mol. Pharmacol., 2006, 70(1), 101-111.

[992] O'Sullivan, S.E.; Tarling, E.J.; Bennett, A.J.; Kendall, D.A.; Randall, M.D. Novel time-dependent vascular actions of Delta9tetrahydrocannabinol mediated by peroxisome proliferator-activated receptor gamma. Biochem. Biophys. Res. Commun., 2005, 337(3), 824-831.

[993] O'Sullivan, S.E.; Sun, Y.; Bennett, A.J.; Randall, M.D.; Kendall, D.A. Time-dependent vascular actions of cannabidiol in the rat aorta. Eur. J .Pharmacol., 2009, 612(1-3), 61-68.

[994] Carroll, C.B.; Zeissler, M.L.; Hanemann, C.O.; Zajicek, J.P. $\Delta^{9}$ tetrahydrocannabinol $\left(\Delta^{9}\right.$-THC) exerts a direct neuroprotective effect in a human cell culture model of Parkinson's disease. Neuropathol. Appl. Neurobiol., 2012, 38(6), 535-547.

[995] Karst, M.; Salim, K.; Burstein, S.; Conrad, I.; Hoy, L.; Schneider, U. Analgesic effect of the synthetic cannabinoid CT-3 on chronic neuropathic pain. JAMA, 2003, 290, 1757-1762.

[996] Karst, M. Comments on "cannabimimetic properties of ajulemic acid". J. Pharmacol. Exp. Ther., 2007, 322(1), 420-421.

[997] Dyson, A.; Peacock, M.; Chen, A.; Courade, J.P.; Yaqoob, M.; Groarke, A.; Brain, C.; Loong, Y.; Fox, A. Antihyperalgesic properties of the cannabinoid CT-3 in chronic neuropathic and inflammatory pain states in the rat. Pain, 2005, 116, 129-137.

[998] Vann, R.E.; Cook, C.D.; Martin, B.R.; Wiley, J.L. Cannabimimetic properties of ajulemic acid. J. Pharmacol. Exp. Ther., 2007, 320, 678-686.

[999] Wiley, J.L. Letter: preclinical assessment of abuse liability of ajulemic acid. Cannabinoids, 2007, 2, 3-4.

[1000] Tepper, M.A.; Zurier, R.B.; Burstein, S.H. Ultrapure ajulemic acid has improved $\mathrm{CB}_{2}$ selectivity with reduced $\mathrm{CB}_{1}$ activity. Bioorg. Med. Chem., 2014, 22(13), 3245-3251.

[1001] Burstein, S.H.; Tepper, M.A. In vitro metabolism and metabolic effects of ajulemic acid, a synthetic cannabinoid agonist. Pharmacol. Res. Perspect., 2013, 1(2), e00017. 
[1002] Mechoulam, R. Plant cannabinoids: a neglected pharmacological treasure trove. Br. J. Pharmacol., 2005, 146(7), 913-915. 


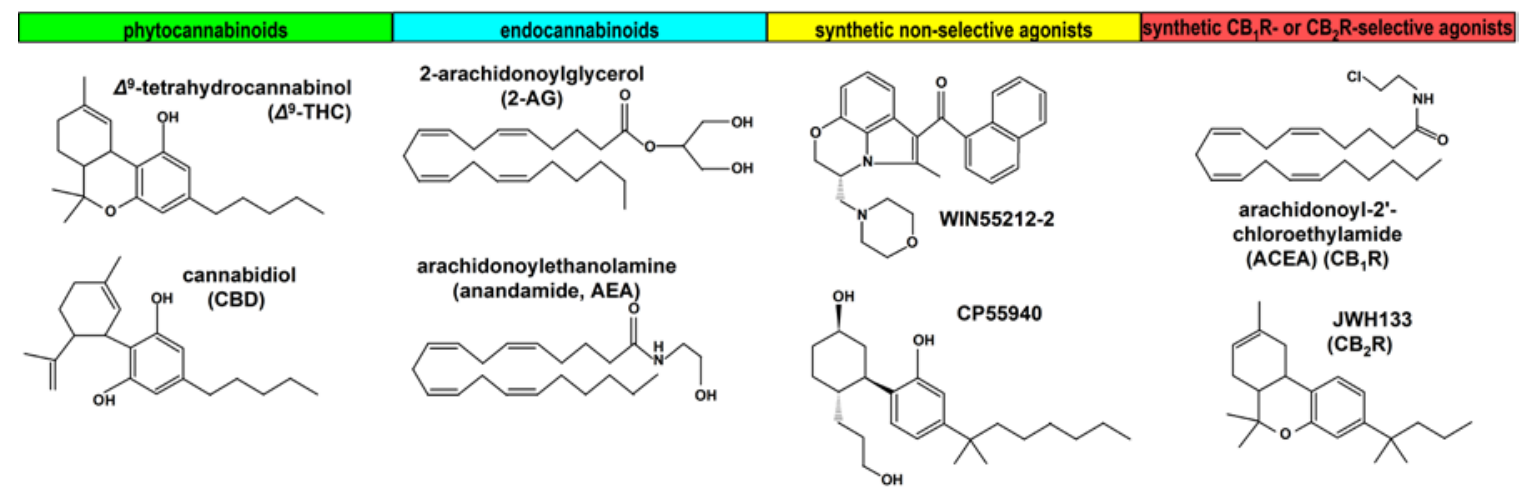

Fig. (1). Chemical structure of important cannabinoid receptor agonists. $1^{\text {st }}$ column: Major phytocannabinoids of the Cannabis plant, $\Delta^{9}$-THC and CBD. $2^{\text {nd }}$ column: The most investigated two endocannabinoids, 2-AG and anandamide. $3^{\text {rd }}$ column: The most efficacious non-selective, synthetic aminoalkylindole WIN55212-2, and the potent non-classical $\Delta^{9}$-THC-analog CP55940. $4^{\text {th }}$ column: The $\mathrm{CB}_{1} \mathrm{R}$-selective, metabolically-resistant synthetic anandamide-analog (eicosanoide) ACEA, and the $\mathrm{CB}_{2} \mathrm{R}$-selective classical synthetic cannabinoid and $\Delta^{9}$-THC-analog, JWH133. 


\section{Fatty acid biosynthesis and/or breakdown}

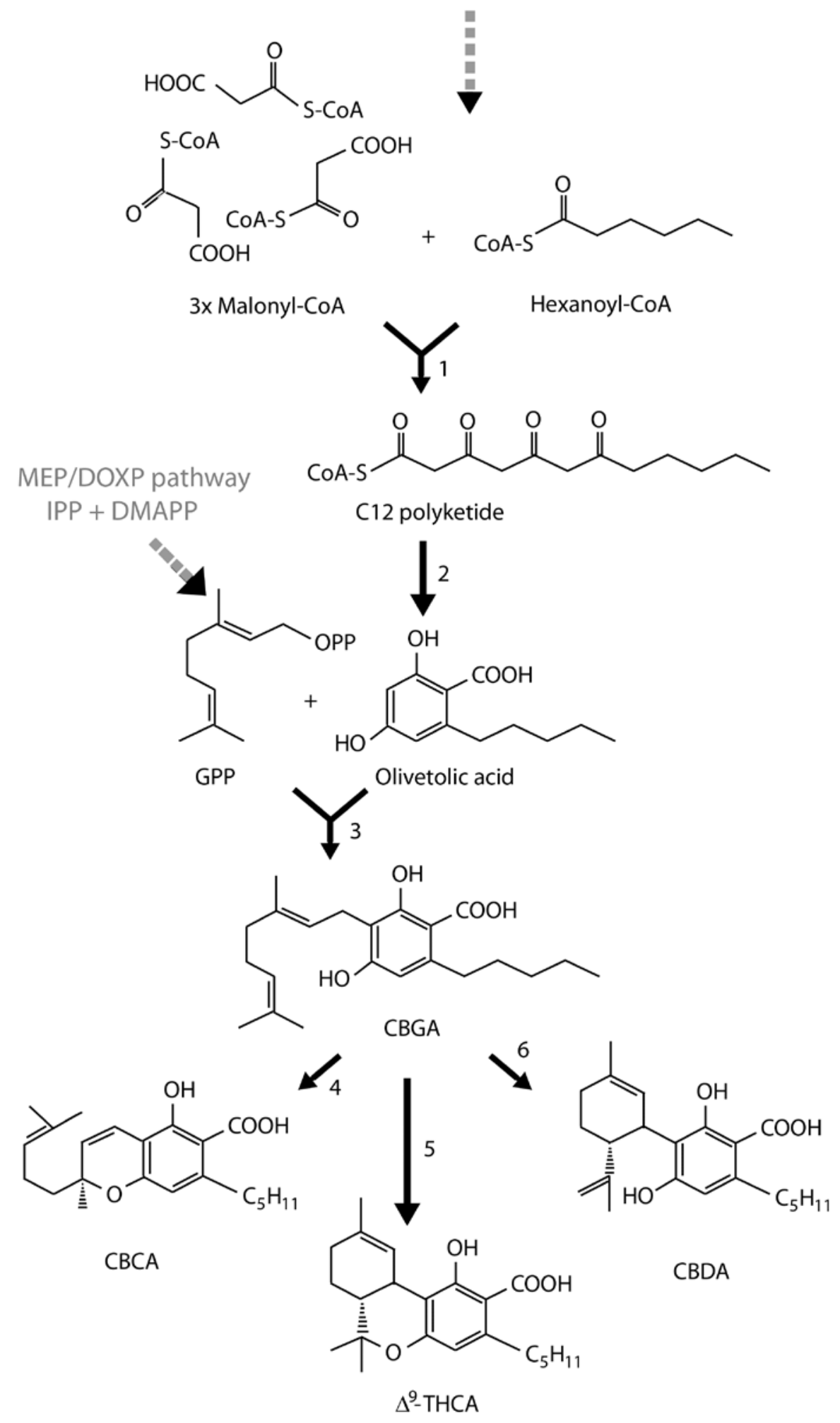

Fig. (2). Outline of the biosynthesis of the major phytocannabinoids. For abbreviations please see the text or the List of Abbreviations. Gray text and dotted gray arrows indicate other metabolic pathways which provide the precursors for cannabinoid biosynthesis. The reactions are catalyzed by the following enzymes: 1. TKS/PKS, 2. OAC, 3. GOT, 4. CBCA synthase, 5. THCA synthase, 6 . CBDA synthase. 

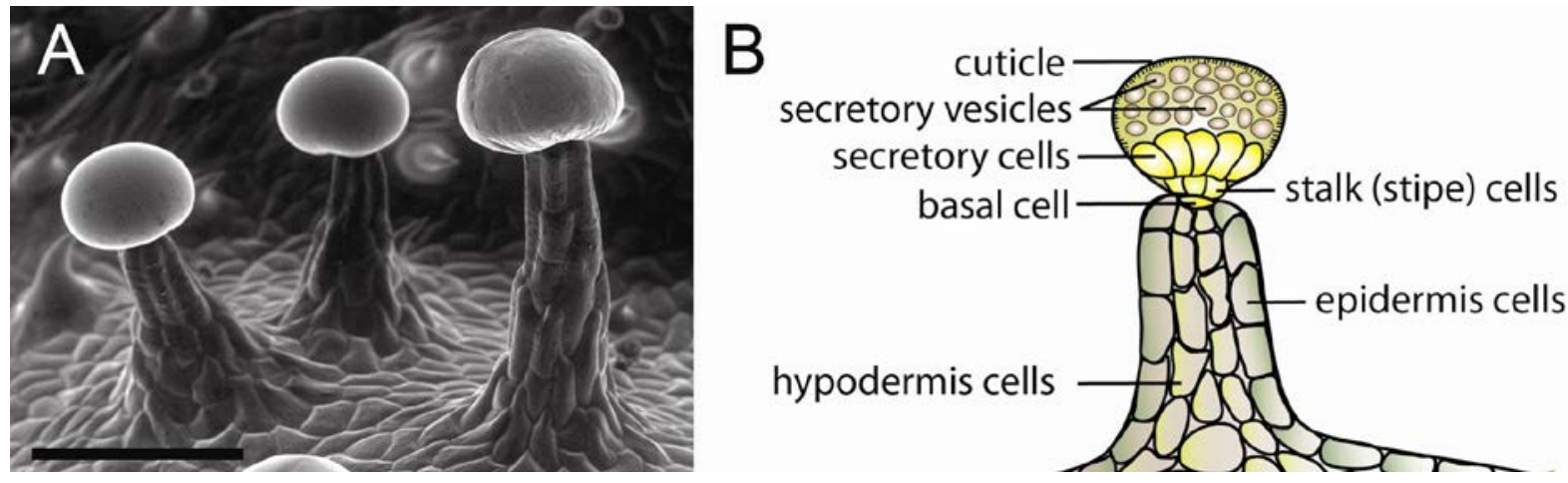

Fig. (3). Capitate-stalked secretory glands of Cannabis. A) Scanning electron micrographs of three capitate-stalked secretory glands on the abaxial epidermis of a perigonal bract (the bract surrounding the pistil) in a drug-type Cannabis sativa strain. Several claw-like unicellular cystolith hairs can be observed in the background. Bar: $100 \mu \mathrm{m}$. Micrograph courtesy to E. Small and T. Antle (Agriculture and Agri-Food Canada, Ottawa). B) Structure of a captitate-stalked secretory gland in cross section. 


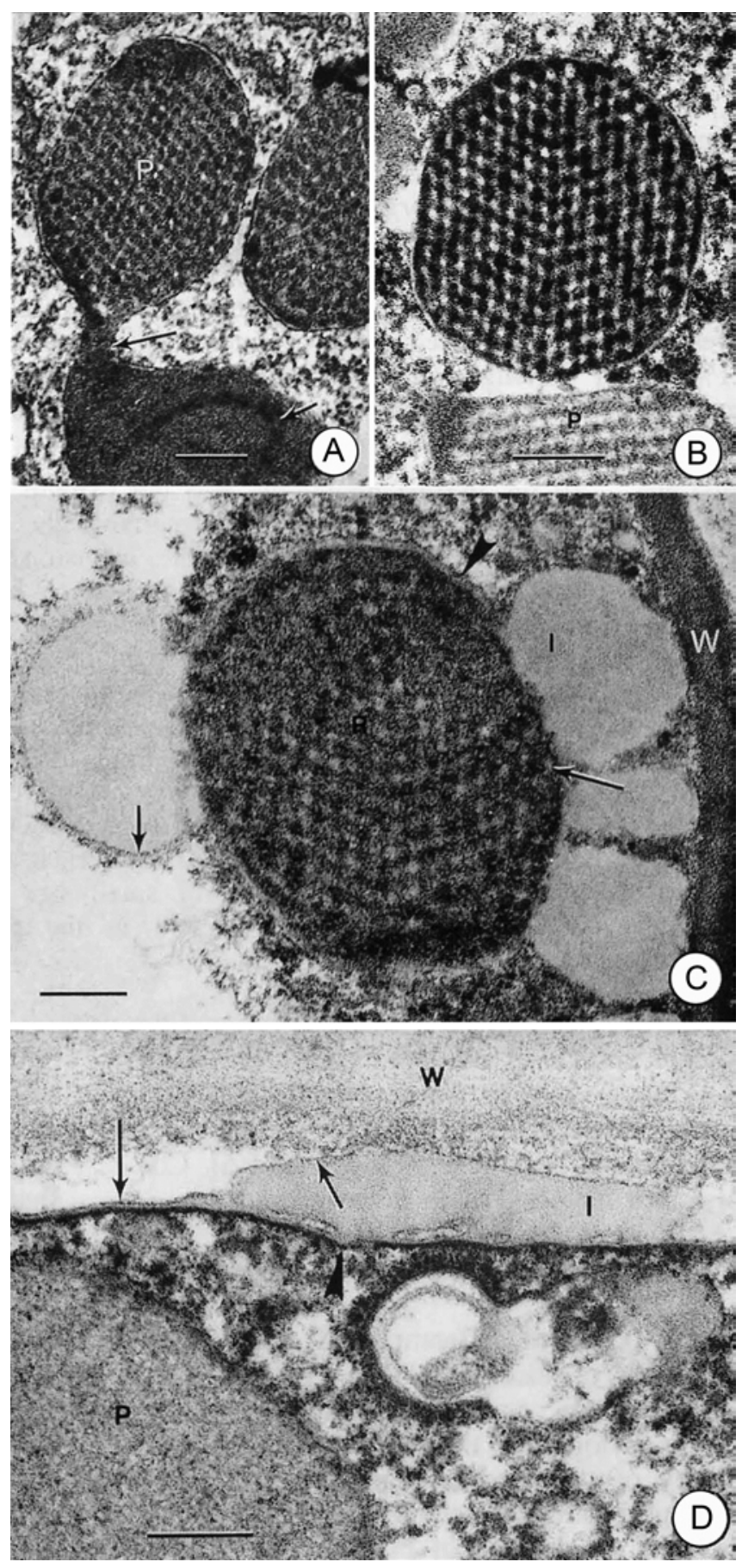

Fig. (4). Transmission electron micrographs showing plastids and the secretory process in Cannabis sativa disc cells from conventionally (chemically fixed) samples (reproduced with permission from [147]). A) Plastid with constriction (long arrow) and two distended regions, the upper containing a paracrystalline reticulate body, the lower containing a thylakoid (short arrow). B) Plastid section containing a reticulate body with different lattice orientations that fills the entire circular plastid section. Another plastid section within the same cell (lower part of the figure) has differently stained membranes of the reticulate body and the stroma. C) Plastid with reticulate body and voluminous inclusions (containing the secreted material) along the envelope surface (arrowhead). D) Plasma membrane (long arrow) showing inclusion (plastid-derived secreted material) positioned in periplasmic space, delimited by a surface (short arrow) and being in contact with the plasma membrane (arrowhead). Bar: $0.2 \mu \mathrm{m}$ (A, B, C) and $0.1 \mu \mathrm{m}$ (D). Unlisted abbreviations: I, inclusion (secreted material); P, plastid; R, reticulate body, $\mathrm{W}$, cell wall. 


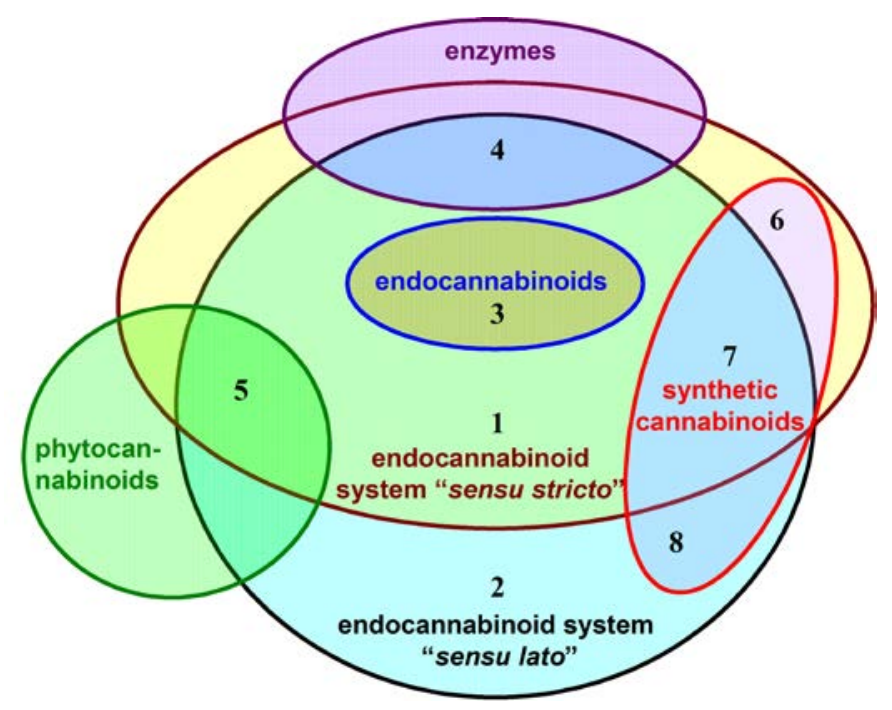

Fig. (5). Overview of the endocannabinoid system. The central oval encompasses the most basic elements of the endocannabinoid system in the narrow sense, i.e., (1) the $\mathrm{CB}_{1}$ and $\mathrm{CB}_{2}$ cannabinoid receptors, as well as their specific adaptor proteins such as the CRIP ${ }_{1 \mathrm{a}}$. Additional receptors may be associated with the endocannabinoid system in the broad sense, such as (2) the GPR18, the GPR55 and the TRPV ${ }_{1} R$. Although these latter three receptors can be activated by lipids which do not bind to the $C_{1} R$ and the $C_{2} R$, other lipid-derivatives such as (3) anandamide and 2-AG are agonists of the endocannabinoid receptors of both the narrow and the broad sense, thus they are called endocannabinoids. The endocannabinoid system also creates intersections with sets of enzymes such as (4) ABDH6/12, COX-2, DAGL $\alpha$, FAAH, MAGL, PLD, which are key components of endocannabinoid signaling but are not exclusive to the endocannabinoid system. Many phytocannabinoids including (5) $\Delta^{9}$-THC and CBD interact with the endocannabinoid system of both the narrow and the broad sense, but the majority of phytocannabinoids (CBN, CBCV, CBGV and so on) do not have submicromolar affinity toward these receptors. They are still called phytocannabinoids because of the plant that produces them and their structural resemblance to $\Delta^{9}$-THC, but not because of their action. Finally, the class of synthetic cannabinoids is fully encircled by the endocannabinoid system of both the narrow and the broad sense, because these ligands are designed on purpose to interact only with cannabinoid receptors. Among them, (6) WIN55212-2 is known to interact only with the $\mathrm{CB}_{1} \mathrm{R}$ and the $\mathrm{CB}_{2} \mathrm{R}$, at least in the nanomolar range. However, many synthetic cannabinoids are potent ligands for the endocannabinoid receptors of both sensu stricto et lato. In fact, some of them are often incorrectly called "selective" to one receptor. This list includes but is not limited to (7) ACEA, ACPA, AM251, CP55940, HU210, HU308, JWH133, and SR141716A. Finally, (8) CID16020046, iodoresiniferatoxin, ML193, O-1602 and O-1918 among many others are ligands for many receptors of the endocannabinoid system of the broad sense, except for the $\mathrm{CB}_{1} \mathrm{R}$ and the $\mathrm{CB}_{2} \mathrm{R}$. For details, see text. For the abbreviations, please consult the List of Abbreviations. 


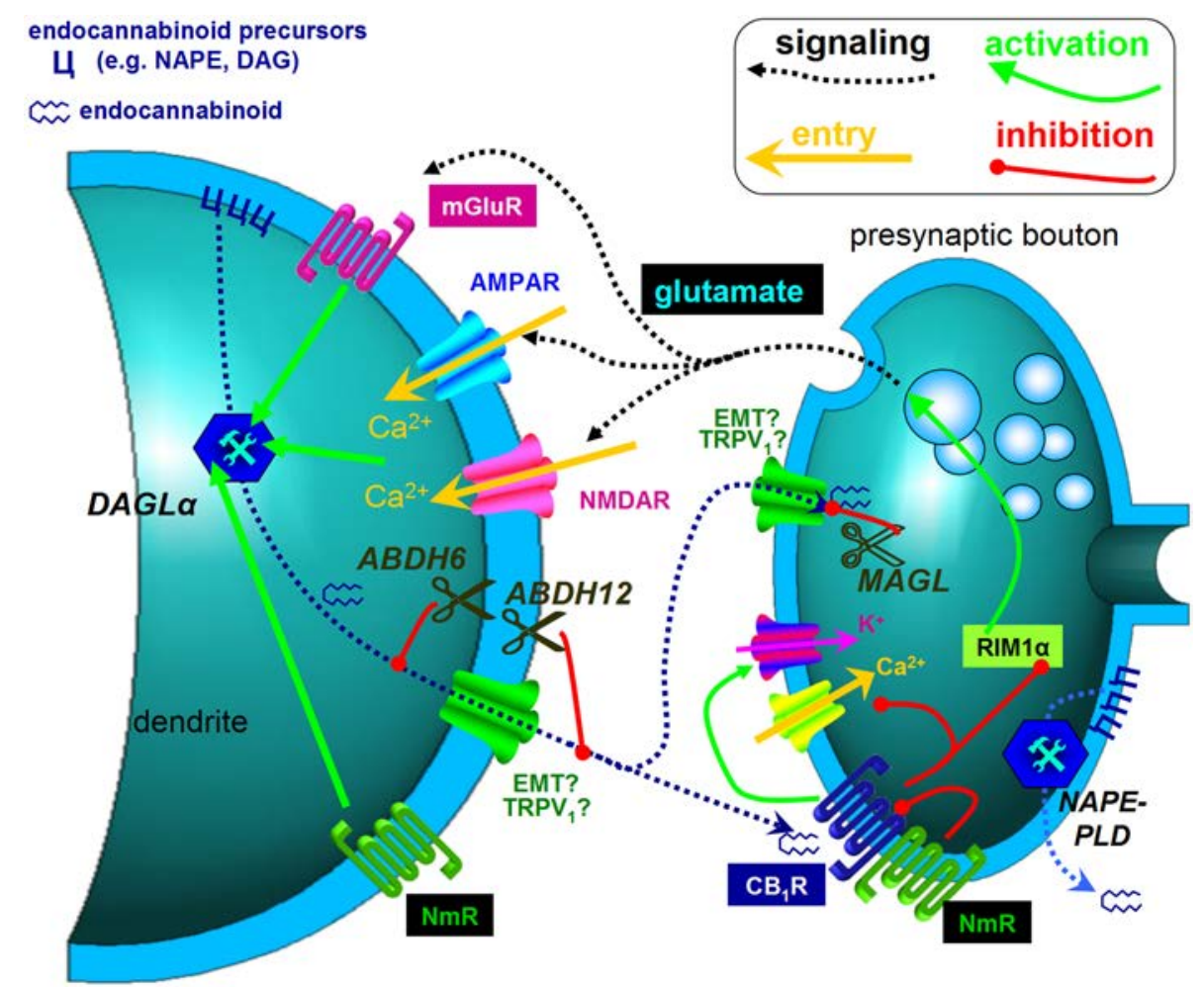

Fig. (6). Schematic illustration of endocannabinoid signaling in a glutamatergic synapse. Note that DAG, DAGL $\alpha$, ABDH6 and ABDH12 as well as MAGL are key components of 2-AG signaling, which is believed to be the major form of retrograde endocannabinoid signaling. NAPE and PLD are also displayed in this figure, both of which are necessary for anandamide signaling, but it is possible that anandamide exerts its effect locally where it is produced, i.e., presynaptically, or in an anterograde fashion such as classical neurotransmitters. NmR stands for a general neuromodulator receptor which can be many of those receptors detailed in the text, including but not limited to $M_{1} R, D_{2} R, 5-H T_{2 A} R, A_{2 A} R$, insulin receptor, or glucocorticoid receptor. Unlisted abbreviations: AMPAR, $\alpha$-amino-3-hydroxy-5-methyl-4-isoxazolepropionic acid receptor; NMDAR, N-methyl-D-aspartic acid receptor. 


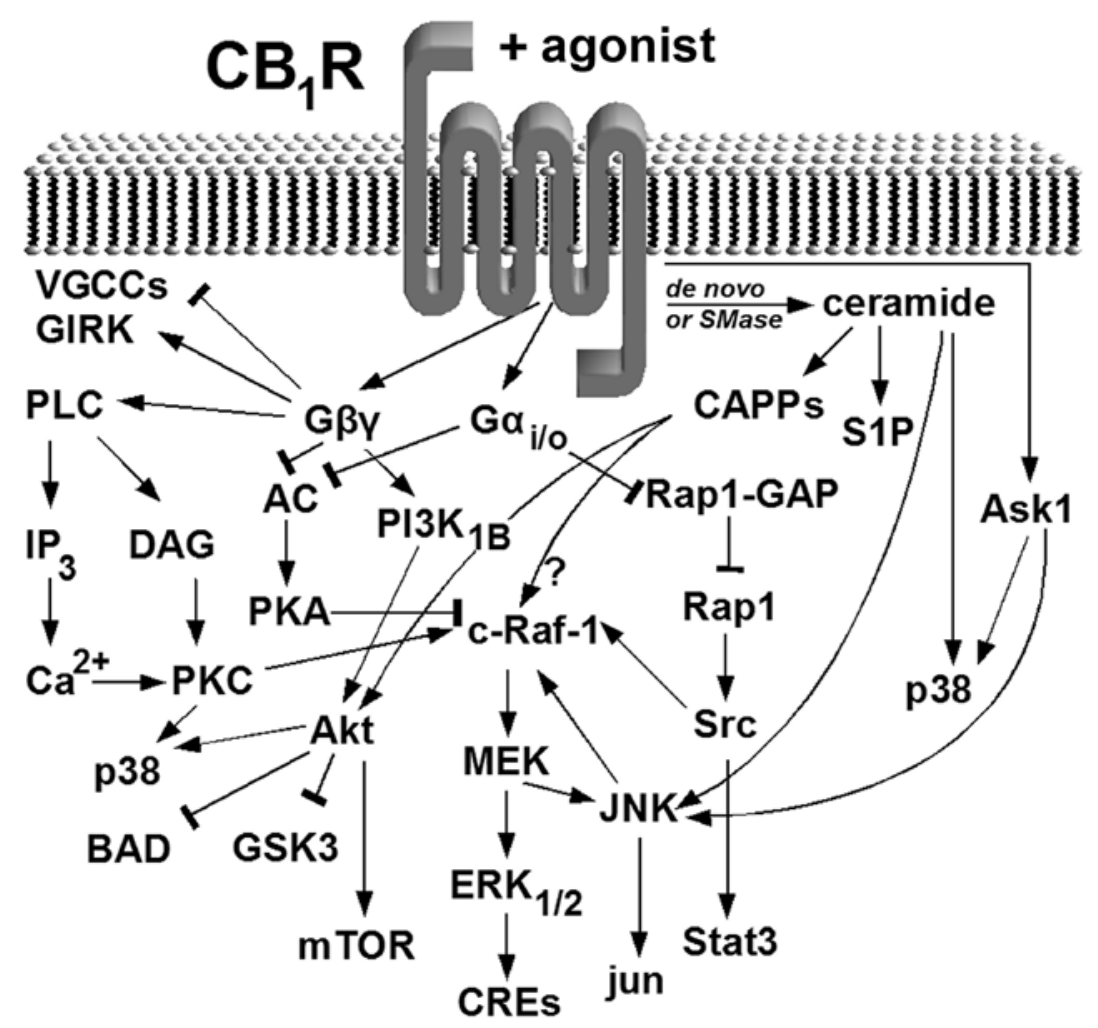

Fig. (7). Major signaling routes of the $C_{1} R$ via $G_{i / o}$ proteins. Note that for sake of simplicity, the $C B_{1} R$ here is represented as a monomer; however, it is thought to exist in the membrane as a homodimer which can even be a part of a heteromultimeric assembly. Abbreviations: AC, adenylyl cyclase; Akt, protein kinase B; Ask1, apoptosis signal-regulating kinase 1; BAD, Bcl-2associated death promoter; c-Raf-1, proto-oncogene serine/threonine-protein kinase, also known as mitogen-activated protein kinase (MAPK) kinase kinase (MAP3K); CAPPs, ceramide-activated protein phosphatases; CREs, cAMP response elements; DAG, 1,2-diacylglycerol; ERK $K_{1 / 2}$, extracellular regulated kinase 1/2; $\mathrm{G}_{\mathrm{i} / \mathrm{o}}, \mathrm{G} \beta \gamma$, dissociated inhibitory alpha subunit binding GTP, and the beta-gamma complex of the activated heterotrimeric inhibitori (i/o) G protein; GAP, GTPase-activating protein;

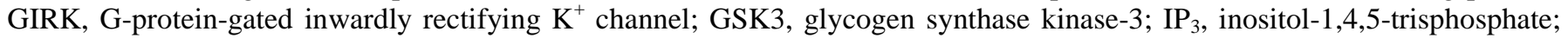
JNK, c-Jun N-terminal kinase; MEK, ERK kinase; mTOR, mammalian target of rapamycin; p38 mitogen-activated protein kinases; $\mathrm{PI}_{3} \mathrm{~K}_{1 \mathrm{~B}}$, class $1 \mathrm{~B}$ phosphoinositide 3-kinase; PKA, protein kinase A; PKC, protein kinase C; PLC, phospholipase C; Rap-1, Ras-proximate-1; S1P, sphingosine 1-phosphate; SMase, sphingomyelinase; Src, proto-oncogene tyrosine-protein C-

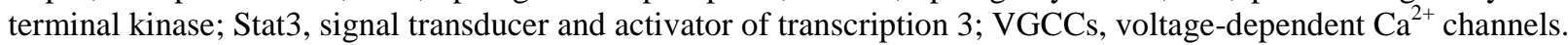




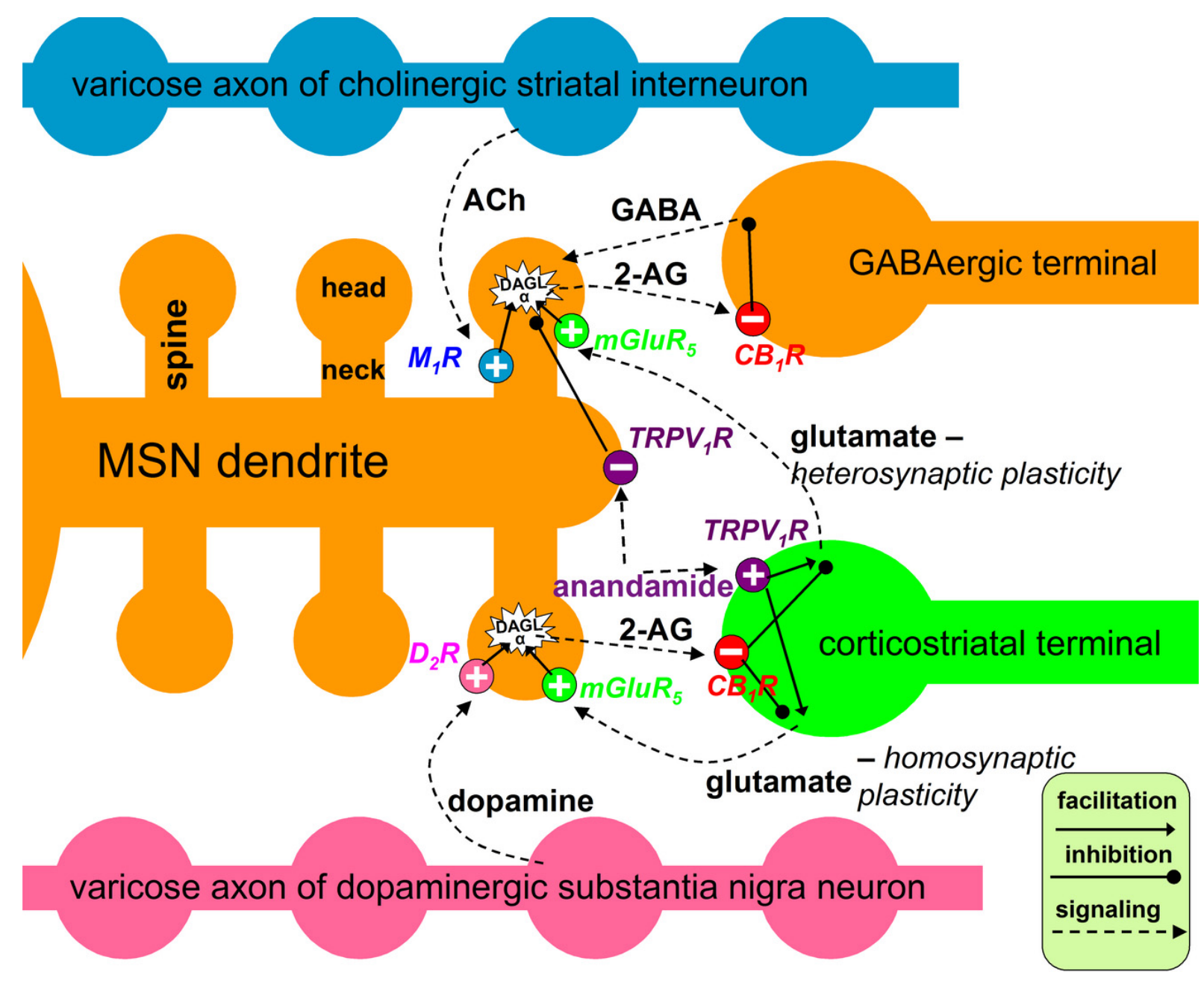

Fig. (8). Simplified scheme of modulation of synaptic transmission and neuromodulation in the dorsolateral striatum. For sake of simplicity, we omitted a few typical features of the striatum that were not essential for the understanding of the figure, including but not limited to adenosinergic signaling, the origin of anandamide, thalamostriatal afferents, or the distinction between direct and indirect pathways nor astrocytes. For the the abbreviations, see or the List of Abbreviations. + in a circle: stimulatory receptor; - in a circle: inhibitory receptor. 


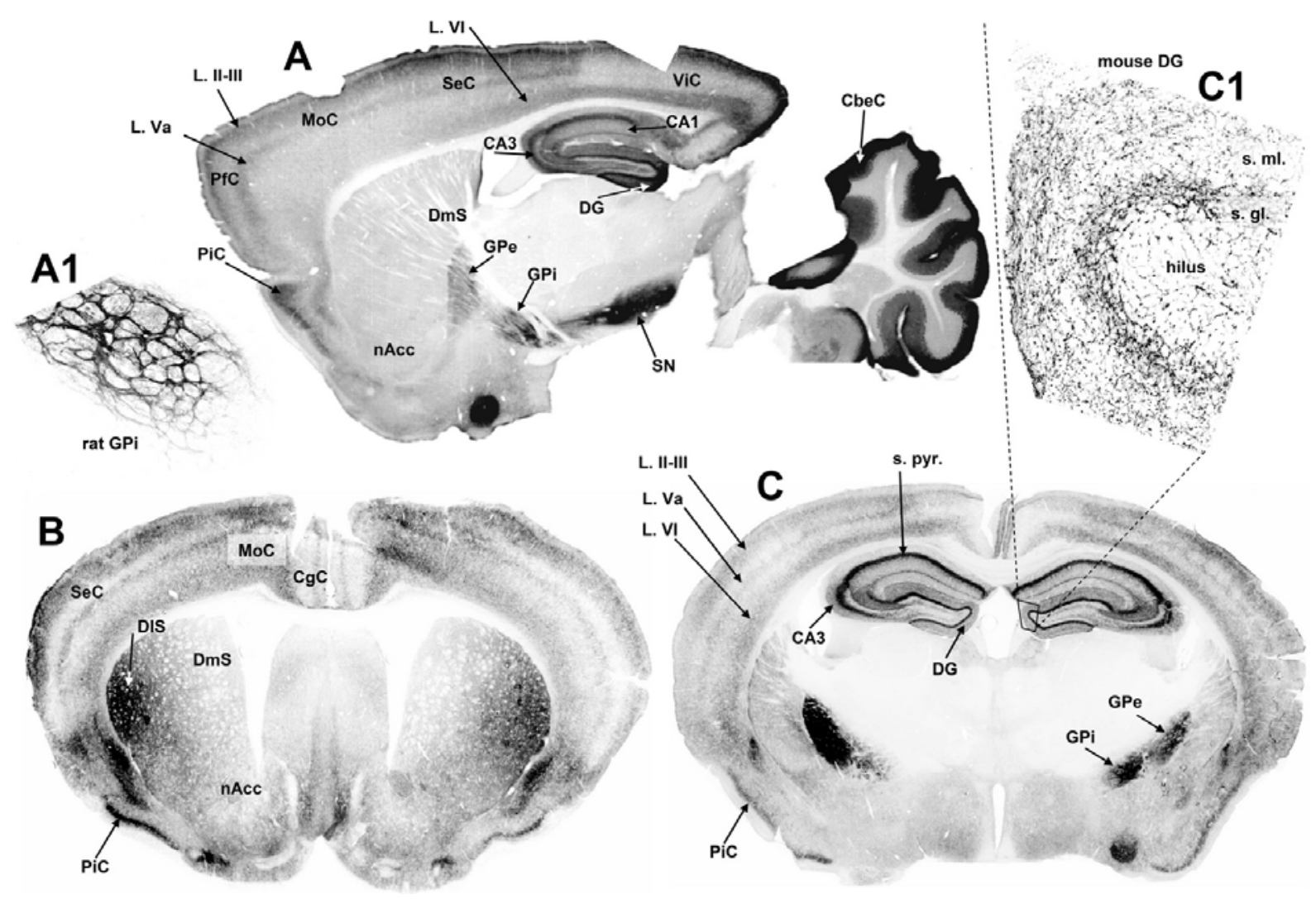

Fig. (9). Fluorescence microscopy images showing the distribution of $\mathrm{CB}_{1} \mathrm{R}$ immunoreactivity in the $40 \mu \mathrm{m}$-thick mediansagittal brain slice of an adult rat (A), and in $30 \mu \mathrm{m}$-thick coronal slices of the brain of an adult C57bl/6 mouse, cut at the level of the striatum (B) and the hippocampus (C). Confocal microscopy images at $63 \times$ magnification representing (A1) a dense and artistic meshwork of $\mathrm{CB}_{1} \mathrm{R}$-positive fibres in the entopeduncular nucleus of the rat, and $(\mathbf{C} \mathbf{1}) \mathrm{CB}_{1} \mathrm{R}$-positive interneurons as well as granule cells in the mouse dentate gyrus. Unlisted abbreviations: CA1, CA3, cornu Ammonis 1 and 3 subfields of the hippocampus; CbeC, cerebellar cortex; CgC, cingulate cortex; L. II, III, Va, VI: different layers of the cerebral cortex; DG, dentate gyrus of the hippocampus; DIS, dorsolateral striatum; DmS, dorsomedial striatum; GPi and GPe, globus pallidus interna and externa; MoC, motor cortex; nAcc, nucleus accumbens; PiC, piriform cortex; PfC, prefrontal cortex; s. gl., stratum granulare; s. ml., stratum moleculare; SN, substantia nigra; s. pyr., stratum pyramidale; SeC, sensory cortex; ViC, visual cortex. Immunostaining was carried out with a guinea pig anti- $\mathrm{CB}_{1} \mathrm{R}$ antibody [552], at 1:200 dilution, and with an antiguinea-pig dylight 594 secondary antibody. For additional details and $\mathrm{CB}_{1} \mathrm{R} \mathrm{KO}$ controls, see [458,553]. For additional details on hippocampal $\mathrm{CB}_{1} \mathrm{R}$ distribution, see $[458,554]$. These previously unpublished immunostainings were kindly provided by Dr. Samira G. Ferreira, Center for Neuroscience and Cell Biology of Coimbra, University of Coimbra, Portugal. All studies were conducted according to the principles and procedures outlined in the EU directive (2010/63/EU) for the care and use of laboratory animals, according to the guidelines of "3Rs" (replacement, reduction, refinement) in the guidelines of EU (86/609/EEC) and FELASA, and were approved by the local Animal Care Committee of the university (license number 025781) and the Portuguese Ministry of Agriculture. Particular care was taken to minimize both animal suffering and the animal number used in each study. The same applies for the experiments shown in Fig. (10). 

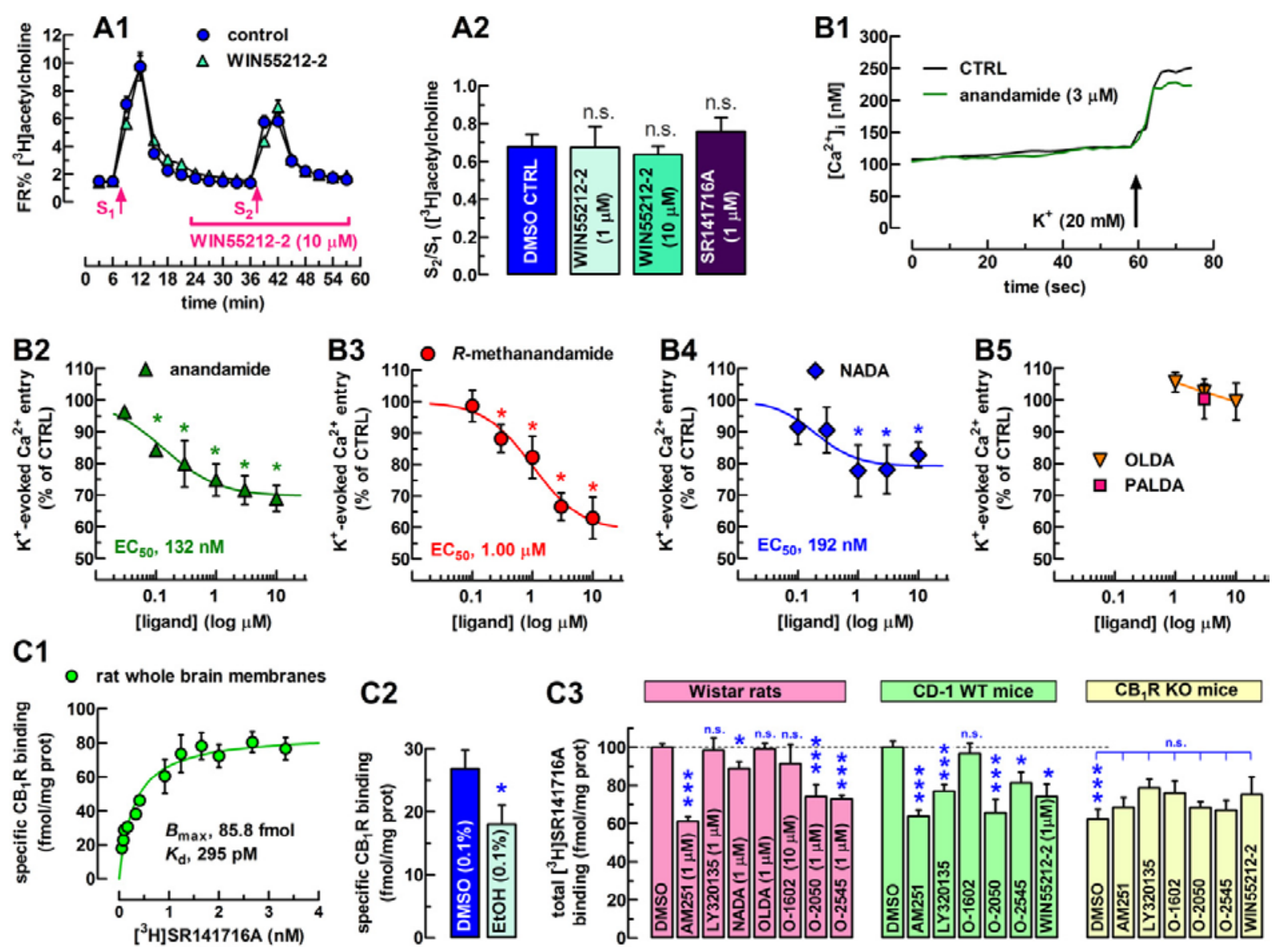

Fig. (10). Previously unpublished neurochemical data supporting the dominance of the $C_{1} R$ in the central effects of cannabimimetics. Panel A1 is a diagram showing the time-course of $\left[{ }^{3} \mathrm{H}\right]$ acetylcholine release values (mean \pm S.E.M. of 4-6 rats) in the presence of hemicholinium-3 $(10 \mu \mathrm{M})$ in rat striatal slices. As indicated by $S_{1}$ and $S_{2}$, the resting efflux was stimulated twice with electrical pulses, too $(40 \mathrm{~V}, 2 \mathrm{~Hz}, 360 \times 1 \mathrm{~ms}$ shocks). WIN55212-2 ( 1 and $10 \mu \mathrm{M})$ as well as SR141716A $(1 \mu \mathrm{M})$ were given 10 min before $S_{2}$, and their mean effect amplitudes $( \pm$ S.E.M) are displayed in panel A2. Experiments were carried out by A.K. For further details, see the methods for similar experiments in [390,554].

Panel $\mathbf{B 1}$ shows the mean ( $\mathrm{n}=8$ rats) effect of anandamide $(3 \mu \mathrm{M})$ treatment on $20 \mathrm{mM} \mathrm{K}^{+}$-evoked $\mathrm{Ca}^{2+}$ entry in striatal nerve terminals. Treatment with anandamide or its vehicle, EtOH started at 0 second. Panels B2, B4 and B5 show that the hybrid $\mathrm{CB}_{1} \mathrm{R} / \mathrm{CB}_{2} \mathrm{R} / \mathrm{GPR} 18 / \mathrm{GPR} 55 / \mathrm{TRPV}_{1} \mathrm{R}$ agonist, anandamide and the hybrid $\mathrm{CB}_{1} \mathrm{R} / \mathrm{TRPV}{ }_{1} \mathrm{R}$ agonist, NADA, but not the selective $\mathrm{TRPV}_{1} \mathrm{R}$ agonist, OLDA or the NADA/OLDA analog, palmytoyldopamine (PALDA) inhibit the depolarization-induced $\mathrm{Ca}^{2+}$ entry in a concentration-dependent fashion. The effect of anandamide is not due to a putative rapid metabolism, as its metabolically stable analog, $R$-methanandamide (B3) is also capable of mimicking the effect of anandamide. For further detalis, see $[391,573]$. Experiments were performed by Dr. Samira G. Ferreira, Center for Neuroscience and Cell Biology of Coimbra, University of Coimbra, Portugal.

Panel C1 displays a $\left[{ }^{3} \mathrm{H}\right]$ SR141716A $\left({ }^{3} \mathrm{H}\right.$-rimonabant) binding isotherm in total brain homogenates of 3-5 rats. Panel C2 calls the attention to our discovery that the presence of already as low as $0.1 \%$ ethanol (EtOH) greatly increases non-specific $\left[{ }^{3} \mathrm{H}\right] \mathrm{SR} 141716 \mathrm{~A}$ binding on the costs of specific $\left[{ }^{3} \mathrm{H}\right] \mathrm{SR} 141716 \mathrm{~A}$ binding, decreasing the latter value. Therefore, DMSO is always recommended to dissolve any ligands to be tested in $\mathrm{CB}_{1} \mathrm{R}$ binding assays. Bovine serum albumin decreases nonspecific binding by harvesting $\left[{ }^{3} \mathrm{H}\right] \mathrm{SR} 141716 \mathrm{~A}$ from the assay medium, hence falsifying the radioligand concentration (not shown). Panel $\mathbf{C} 3$ shows that AM251 $(1 \mu \mathrm{M})$ is the best choice to measure specific $\left[{ }^{3} \mathrm{H}\right] \mathrm{SR} 141716 \mathrm{~A}$ binding at $\mathrm{CB}_{1} \mathrm{Rs}$ in the brain, and that at the used concentration of $1 \mathrm{nM},\left[{ }^{3} \mathrm{H}\right] \mathrm{SR} 141716 \mathrm{~A}$ binding was exclusively $\mathrm{CB}_{1} \mathrm{R}$-dependent, showing no evidence for the involvement of other receptors including GPR18, GPR55 or TRPV ${ }_{1} \mathrm{R}$ in the total binding of $\left.{ }^{3} \mathrm{H}\right] \mathrm{SR} 141716 \mathrm{~A}$. Experiments were carried out by A.K. For additional details, see [396,553]. 